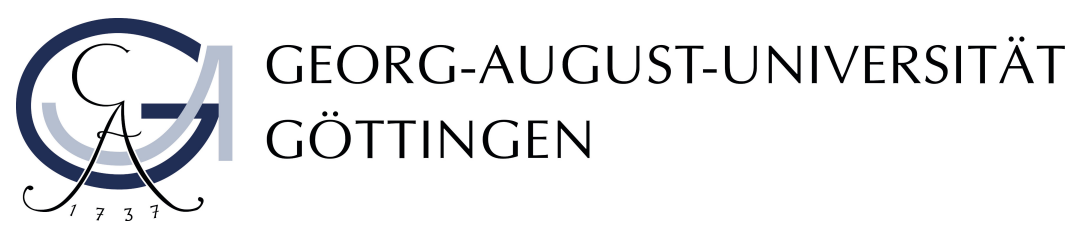

\title{
Radiative Corrections in Curved Spacetime and Physical Implications to the Power Spectrum and Trispectrum for different Inflationary Models
}

\author{
Dissertation \\ zur Erlangung des mathematisch-naturwissenschaftlichen \\ Doktorgrades
}

Doctor rerum naturalium

der Georg-August-Universität Göttingen

Im Promotionsprogramm PROPHYS

der Georg-August University School of Science (GAUSS) vorgelegt

Von

Simone Dresti

aus Locarno

Göttingen, 2018 


\section{Betreunngsausschuss:}

Prof. Dr. Laura Covi, Institut für Theoretische Physik, Universität Göttingen

Prof. Dr. Karl-Henning Rehren, Institut für Theoretische Physik, Universität Göttingen

Prof. Dr. Dorothea Bahns, Mathematisches Institut, Universität Göttingen

Miglieder der Prüfungskommission:

Referentin: Prof. Dr. Laura Covi, Institut für Theoretische Physik, Universität Göttingen Korreferentin: Prof. Dr. Dorothea Bahns, Mathematisches Institut, Universität Göttingen

Weitere Mitglieder der Prüfungskommission:

Prof. Dr. Karl-Henning Rehren, Institut für Theoretische Physik, Universität Göttingen

Prof. Dr. Stefan Kehrein, Institut für Theoretische Physik, Universität Göttingen

Prof. Dr. Jens Niemeyer, Institut für Astrophysik, Universität Göttingen

Prof. Dr. Ariane Frey, II. Physikalisches Institut, Universität Göttingen

TAG DeR mündLichen PrüFung: Mittwoch, 23. Mai 2018 
Ai miei nonni Marina, Palmira e Pierino 
In a quantum field theory with a time-dependent background, as in an expanding universe, the time-translational symmetry is broken. We therefore expect loop corrections to cosmological observables to be time-dependent after renormalization for interacting fields.

In this thesis we compute and discuss such radiative corrections to the primordial spectrum and higher order spectra in simple inflationary models. We investigate both massless and massive virtual fields, and we disentangle the time dependence caused by the background and by the initial state that is set to the Bunch-Davies vacuum at the beginning of inflation.

For the investigated models, we find that the radiative corrections to the primordial spectrum result in oscillatory features that are not present at tree-level. These features are also present in higher order spectra and depend on the initial conditions of the theory. In all the investigated cases the departure from near-scale invariance and from Gaussianity is very small and it is in full agreement with the current Planck constraints.

Future cosmic microwave background measurements may improve the current limits on feature-full primordial spectra, giving the hope to observe these effects in the scenario of hybrid inflation.

Keywords: Primordial Power Spectrum, Trispectrum, Inflationary Perturbations, Inflaton Field, Slow-Roll Inflation, Hybrid Inflation, Chaotic Inflation, Non-Linearity Parameter, De-Sitter Spacetime, Renormalization in Curved Spacetime, Radiative Corrections, Finite Time Contributions, CTP Formalism, WKB Propagator, Hypergeometric Propagator 
1 Introduction $\quad 1$

2 Cosmic inflation 3

2.1 The Friedmann Lemaître Robertson Walker universe . . . . . . . . . . 3

2.2 Motivations for inflation . . . . . . . . . . . . . . . 7

2.3 Slow-roll inflation . . . . . . . . . . . . . . . . . . . . 8

2.4 Inflationary perturbations . . . . . . . . . . . . . . . . . . . 10

2.5 Selection of classes of models . . . . . . . . . . . . . . . . . . 15

3 Quantum field theory in curved spacetime 19

3.1 Introduction . . . . . . . . . . . . . . . . . . 20

3.2 Renormalization in Minkowski spacetime . . . . . . . . . . . . . 22

3.3 Adiabatic renormalization . . . . . . . . . . . . . . . 24

3.4 From distribution theory to the Epstein-Glaser renormalization . . . . 28

4 The Schwinger and Keldysh formalism $\quad 37$

4.1 Introduction . . . . . . . . . . . . . . . . . . . . . . . . 38

4.2 Perturbation theory . . . . . . . . . . . . . . . . . . . 40

4.3 CTP propagators . . . . . . . . . . . . . . . . . . . . . 42

5 Renormalization in the CTP formalism 49

5.1 The equal-time two-point correlation function . . . . . . . . . . . 50

5.2 The equal-time four-point correlation function . . . . . . . . . . . . 59

5.3 Massive WKB counter-term in de-Sitter spacetime . . . . . . . . . . . 74

5.4 Counter-terms in the adiabatic regularization scheme . . . . . . . . . 76 
6 Interaction profile and the adiabatic limit $\quad 81$

6.1 Profile dependence of the two-point correlation function . . . . . . . . . 82

6.2 Proper definition of the adiabatic limit of the tadpole . . . . . . . . 89

7 Cosmological applications of renormalization in de-Sitter spacetime 93

7.1 Classical dynamics of the inflationary models . . . . . . . . . . . . . 93

7.2 Radiative corrections to the primordial power spectrum . . . . . . . . 101

7.3 One-loop corrections to the power spectrum with a monomial interaction 103

7.4 One-loop corrections to the power spectrum in hybrid inflation . . . . . 104

7.5 The non-linearity parameter $\tau_{\mathrm{NL}} \ldots \ldots \ldots \ldots$. . . . . . . . . . . . . . . . 112

8 Summary and conclusions $\quad 115$

$\begin{array}{lr}\text { Appendix A Feynman rules } & 119\end{array}$

A.1 $\lambda \phi^{4}$-theory . . . . . . . . . . . . . . . . . . . 119

A.2 Hybrid model . . . . . . . . . . . . . . . . . 121

$\begin{array}{ll}\text { Appendix B Useful integrals } & 123\end{array}$

$\begin{array}{ll}\text { Appendix C Various results } & 125\end{array}$

$\begin{array}{ll}\text { Bibliography } & 135\end{array}$

$\begin{array}{ll}\text { Acknowledgements } & 147\end{array}$

$\begin{array}{ll}\text { Curriculum Vitæ } & 155\end{array}$ 
2.1 Example of a slow-roll single-field inflation . . . . . . . . . . . . . 16

3.1 Cutoff function $\theta_{n}(x)$ for different parameters . . . . . . . . . . . 33

3.2 Singular structure of different propagators in Minkowski spacetime . . . 36

4.1 Closed-time contour $\mathcal{C}$ representing the time-ordering $T_{\mathcal{C}} \ldots \ldots$. . . . . 39

4.2 Hadamard propagators derived from the hypergeometric function for different masses . . . . . . . . . . . . . . . . . . . . 45

4.3 Comparison of the massive Hadamard propagator reconstructed from the hypergeometric function and the WKB propagator . . . . . . . . 47

5.1 Tree-level equal-time two-point correlation function . . . . . . . . . . 50

5.2 Tadpole diagram and its counter-term for a quartic self-interacting scalar field theory in Minkowski spacetime . . . . . . . . . . 51

5.3 Tadpole diagram and its counter-term for a quartic self-interacting scalar field theory in de-Sitter spacetime . . . . . . . . . . . 55

5.4 Cosine integral and sine integral . . . . . . . . . . . . . . 58

5.5 Time dependence of the renormalized two-point correlation function in de-Sitter spacetime . . . . . . . . . . . . . . . . 59

5.6 Tree-level contributions $T_{1}, T_{2}$ and $T_{1}+T_{2}$ from the massive propagators in Minkowski spacetime . . . . . . . . . . . . 61

5.7 Tree-level contributions $T_{1}, T_{2}$ and $T_{1}+T_{2}$ from the massless propagators in de-Sitter spacetime . . . . . . . . . . . . . . 63

5.8 Fish diagrams $A_{1}$ and $A_{2} \ldots \ldots \ldots \ldots$. . . . . . . . 64

5.9 Tree-level and one-loop contributions to the four-point function . . . . 73 
5.10 Mass dependence of the amputated tadpole diagram after renormalization with the WKB couter-term . . . . . . . . . . . . . . 75

5.11 Relative difference between the Hypergeometric and the WKB propagator for a scalar field of mass $60 H \ldots$. . . . . . . . . . . . 76

6.1 Interaction profiles with different extensions during the transition . . . 82

6.2 Renormalized two-point function using different continuous interaction profiles $\lambda(t) \ldots \ldots \ldots \ldots \ldots$. . . . . . . . . . . . . . 84

6.3 Switching-on profile constructed from the arc-tangent function with different parameters . . . . . . . . . . . . . . . . . . . . . 84

6.4 Renormalized two-point function using the arc-tangent interaction profile 85

6.5 Interaction profile dependence of the renormalized tadpole diagram in de-Sitter spacetime . . . . . . . . . . . . . . . . . . . . . . 88

6.6 One-parameter family of test functions $f_{s}(t)$ for the adiabatic limit . . 90

6.7 Time-evolution of the tadpole amplitude for different test functions $f_{s}(t) \quad 90$

6.8 Tadpole amplitude as a function of the continuous parameter $s$ in $f_{s}(t)$ for a fixed time . . . . . . . . . . . . . . . . . . . . . . . 91

7.1 Relative difference and power spectrum for a scalar field theory with monomial interaction . . . . . . . . . . . . . . . . . . . . . 104

7.2 Oscillatory contribution arising from the external propagators . . . . . 106

7.3 Relative difference and renormalized power spectrum for hybrid inflation 108

7.4 Relative difference and renormalized power spectrum for the hybrid supersymmetric model . . . . . . . . . . . . . . . . . . . . . . . . . 109

7.5 Relative difference and renormalized power spectrum for the spectator field model . . . . . . . . . . . . . . . . . . . . . . . . . . . 111

7.6 Non-linearity parameter $\left|\tau_{\mathrm{NL}}\right|$ calculated from the contribution $T_{1}+T_{2}$ for a $\lambda \phi^{4}$-theory . . . . . . . . . . . . . . . . . . . 113 
2.1 Solutions of the Einstein equation for a spatially flat universe dominated by a de-Sitter era or by a non-relativistic matter or radiation epoch . . 6

5.1 Inequivalent Feynman diagrams for the tree-level contributions to the four-point function from a massive scalar field in Minkowski spacetime 60

5.2 Inequivalent Feynman diagrams for the tree-level contributions to the four-point function from a (nearly) massless scalar field in de-Sitter spacetime . . . . . . . . . . . . . . . . . 62

5.3 Inequivalent Feynman diagrams for the one-loop correction to the fourpoint function . . . . . . . . . . . . . . . . 66 66

A.1 Feynman rules for the $\lambda \phi^{4}$ theory . . . . . . . . . . . . . . . 120

A.2 Feynman rules for a two scalar field theory . . . . . . . . . . . . . . . . 122 


\section{CHAPTER 1}

$$
\text { I }
$$

Cosmic inflation [1-3] is a theory that describes an accelerating period of expansion of the primordial universe. It is celebrated as one of the most successful paradigms in cosmology. The success has come through relating the primordial fluctuations [4, 5] to observational data as the temperature anisotropies of the cosmic microwave background or the observed large scale structure of the universe. Moreover, inflation provides a solution to many issues that existed before the 1980s, e.g. to understand the homogeneity and the flatness of our universe.

The spectrum of the primordial perturbations of the curvature tensor is predicted to be nearly scale invariant in the slow-roll scenario and the current observations [6] are consistent with the predictions of a simple single-field inflationary model $[7,8]$.

The classical dynamics of different inflationary scenarios has been studied in the last 40 years. Many effects were already addressed in the past for different models [9-16], including non-gaussianities [17, 18] or oscillatory features in the primordial spectrum [19]. Less attention has been devoted to the understanding of the radiative corrections to the correlation functions of the inflaton field. Since Lorentz invariance is broken because of the expansion of the universe, one expects an intrinsic time dependence in the quantum corrections arsing from the evolving background.

The main goal of this thesis is to investigate the time dependence of the one-loop radiative corrections to the two- and four-point correlation functions that arises both because of the background evolution and the initial conditions. The natural theoretical framework is the Schwinger and Keldysh formalism, where the time-evolution of expectation values is easily calculated, including quantum effects. In this scenario the system is supposed to be in the Bunch-Davies [20] vacuum at the beginning of inflation, giving periodic contributions to the physical observables. The initial time dependence 
is investigated by testing the correlation functions with different interaction profiles and by addressing the question of the adiabatic limit. The issue how to consistently define counter-terms [21-38] in order to absorb the UV divergences is also addressed, together with the problem of IR divergences [39].

In the second part of the thesis the radiative corrections to the correlation functions are applied to explicit inflationary models. The first example is a scalar field theory with quartic self-interaction [40] which is one of the most studied models in inflation (despite being excluded by the present bounds on the tensor-to-scalar ratio [6]). This discussion is extended to models inspired by the hybrid scenario [41], where the dynamics of the inflaton field is influenced by the presence of a heavy field that gives a richer phenomenology.

The shape of the radiative corrections to the primordial spectrum and to higher order spectra $[42,43]$ are predicted for different classes of inflationary models. It is expected that the time dependence of the correlation functions leads to periodic features in the spectra. The trispectrum has an initial time dependence also at tree level.

Indeed, the study of features in the primordial spectrum and trispectrum is a powerful tool to confirm the quantum nature of the inflation field and to discriminate among the vast landscape of inflationary models.

The first chapters are devoted to the theoretical concepts relevant for this work. In Chapter 2 the basic notions of cosmic inflation are presented. In Chapter 3 we start with a brief introduction of quantum field theory in curved spacetime addressing the problem of renormalization. In Chapter 4 the Schwinger and Keldysh formalism is introduced and the theoretical tools needed for perturbation theory are presented. The following chapters are related to the research that we carried on. In Chapter 5 the results about renormalization of the two- and four-point correlation functions are presented. Particular attention is paid to the understanding of the origin of the time dependence of our results. Indeed, in Chapter 6 the interaction profile dependence of the correlation functions and the adiabatic limit is discussed. In Section 7 the radiative corrections to the two- and four-point functions are applied to the primordial spectrum and trispectrum, and an estimate to the cosmological parameter $\tau_{\mathrm{NL}}$ is given. This thesis ends with some concluding remarks in Chapter 8. 


\section{CHAPTER 2}

COSMIC INFLATION

Standard cosmology [44-47] is a modern theory that addresses fundamental questions as the formation and the evolution of the universe and gives an interpretation of the astronomical observations. Before the 1980s, there were however certain questions ${ }^{1}$ that had to be resolved. They were not genuine inconsistencies; the hot big bang cosmology never had the intent to explain the physics of the very early universe. The missing part at the epoch was a theory of the primordial universe that could explain the initial conditions required by the big bang expansion.

In the 1980s [1], it was realized that the initial-condition puzzle could be solved with the idea that during the primordial stage, the universe had a period of fast acceleration called inflation [1-3, 48].

\subsection{The Friedmann Lemaître Robertson Walker uni- verse}

The theoretical framework that allows a quantitative and qualitative description of an expanding universe is given by the Friedmann Lemaître Robertson Walker universe [4956] (named also FRW model). It is based on the simple cosmological principle [57] that at sufficiently large scale, the universe becomes homogeneous and isotropic.

The dynamic of the FRW expanding universe is described by the Einstein's equations $[58-62]$

$$
G_{\mu \nu}:=R_{\mu \nu}-\frac{1}{2} g_{\mu \nu} \mathcal{R}=\frac{1}{M_{P}^{2}} T_{\mu \nu}
$$

\footnotetext{
${ }^{1}$ The most often mentioned are the horizon problem, the flatness problem and the large-scale structure problem.
} 
where $g_{\mu \nu}$ denotes the spacetime metric while $R_{\mu \nu}$ and $\mathcal{R}$ are the Ricci tensor and Ricci scalar, and $M_{P}$ is the reduced Planck mass. This equation describes how matter and radiation (the right-hand side of eq. (2.1)) influences the spacetime background (the left-hand-side) and the other way around.

The cosmological principle gives a simple form for the metric. The physical distance $d s$ between two infinitesimally close comoving spherical coordinates $(r, \theta, \phi)$ and $(r+$ $d r, \theta+d \theta, \phi+d \phi)$ reads

$$
d s^{2}=d t^{2}-a^{2}(t)\left[\frac{d r^{2}}{1-\kappa r^{2}}+r^{2} d \theta^{2}+r^{2} \sin ^{2} \theta d \phi^{2}\right],
$$

where $t$ is the physical time and $\kappa$ is a constant that can be chosen to be 1,0 or -1 for a space with positive, zero or negative spatial curvature. The scalar $a(t)$ is a parameter that depends on time and quantifies the relative expansion of the universe. An accelerating and expanding universe means a positive first and second derivative of the scale factor, i.e. $\dot{a}(t)>0$ and $\ddot{a}(t)>0$.

The famous Hubble law $[63,64]$, which is the first important result supporting the idea of an expanding universe, is related to the derivative of the scale factor. Indeed, the Hubble rate $H(t)$ is defined as

$$
H(t)=\frac{\dot{a}(t)}{a(t)}
$$

A strong prediction of inflation is the fact that the universe should be spatially flat, which is described by the FRW metric (2.2), setting $\kappa=0$. In the semi-classical approach of quantum field theory the metric is considered as a classical background. The time dependence of the scale factor breaks the Poincaré symmetry of the expanding universe. The appropriate theoretical framework to study expectation values for time-dependent setups is the Schwinger and Keldysh formalism and is discussed in Chapter 4.

Often it is useful to write the metric in conformal time as (for $\kappa=0$ )

$$
d s^{2}=a^{2}(\tau)\left[d \tau^{2}-d r^{2}-r^{2} d \theta^{2}-r^{2} \sin ^{2} \theta d \phi^{2}\right]
$$

where we defined

$$
d \tau=\frac{d t}{a(t)} \Rightarrow \tau=\tau_{\text {in }}+\int_{t_{\text {in }}}^{t} \frac{d t}{a(t)} .
$$

The assumption of the cosmological principle simplifies the Einstein equation (2.1) 
into a set of two independent relations called the Friedmann equations

$$
\begin{aligned}
H^{2} & =\frac{\dot{a}^{2}}{a^{2}}=\frac{\rho}{3 M_{P}^{2}}-\frac{\kappa}{a^{2}}, \\
\dot{H}+H^{2} & =\frac{\ddot{a}}{a}=-\frac{1}{6 M_{P}^{2}}(\rho+3 p),
\end{aligned}
$$

where $p$ is the pressure and $\rho$ is the energy density. The first expression directly gives the value for the Hubble parameter, and the second the acceleration $\ddot{a}$.

Through the second Friedmann equation in (2.6) one can relate the condition of an accelerating universe to the requirement of a negative pressure

$$
p<-\frac{\rho}{3}<0
$$

In order to study the theoretical properties of an inflationary universe, it is convenient to examine the particular case where the pressure $p$ is exactly given by $-\rho$. This case is called a de-Sitter universe $[45,65-68]$ and is one of the most studied cosmological backgrounds. This is analogous to assume that in the slow-roll approximation $\rho$ and $H$ are constant. In a de-Sitter spacetime the conformal time assumes the simple form

$$
a(\tau)=-\frac{1}{H \tau}
$$

where $\tau_{\text {in }}$ has been chosen such that $t=\infty$ corresponds to $\tau=0$. Therefore the conformal time is always negative.

Let's assume a simple linear relationship between the pressure $p$ and the energy density $\rho$

$$
p=w \rho,
$$

where $w$ is the equation of state parameter. For a de-Sitter universe we already found that $w=-1$. For other values of $w$ one has

$$
a(t) \propto t^{\frac{2}{3(1+w)}}, \quad \text { for } w \neq-1
$$

In a non-relativistic matter dominated era the pressure is vanishing, therefore $p=0$ and $w=0$. On the other hand for a radiation dominated universe the energy momentum tensor is traceless and therefore the equation of state is given by $\rho=3 p$, which means a value of the equation of state parameter equals to 1/3. In Table 2.1 all the solutions are summarized.

In the previous examples the scale factor is estimated for systems where only one component of the matter-energy field content contributes. In order to have a more 
Table 2.1: Solutions of the Einstein equation for a spatially flat universe dominated by a de-Sitter era or by a non-relativistic matter (MD) or radiation (RD) epoch.

\begin{tabular}{c|c|c} 
& $w$ & $a(t)$ \\
\hline de-Sitter & -1 & $e^{H t}$ \\
MD & 0 & $t^{2 / 3}$ \\
RD & $1 / 3$ & $t^{1 / 2}$ \\
\hline
\end{tabular}

realistic scenario one has to consider the case where all the components can contribute to the pressure and to the energy density. Let $p$ and $\rho$ be given by

$$
\begin{aligned}
& p=\sum_{i} p_{i}, \\
& \rho=\sum_{i} \rho_{i},
\end{aligned}
$$

where the index $i$ spans all the fields content of the theory. Let's define the parameter $\Omega$ that represents the energy density ratio today with respect to the critical energy density ${ }^{2} \rho_{\text {crit }}=3 H^{2} M_{P}^{2}$ as

$$
\Omega_{i}=\frac{\rho_{i}\left(t_{0}\right)}{\rho_{\text {crit }}}
$$

By assuming that each component has an equation of state given by eq. (2.9), and assuming that the scale factor today is normalized to 1 , the Friedmann equation becomes

$$
\frac{H^{2}}{H_{0}^{2}}=\sum_{i} \Omega_{i} a^{-3\left(1+w_{i}\right)}+\Omega_{\kappa} a^{-2},
$$

where the last term is the curvature density parameter

$$
\Omega_{\kappa}=-\frac{\kappa}{H^{2} a^{2}\left(t_{0}\right)}
$$

By evaluating eq. (2.13) today, one finds the so-called golden rule of cosmology, i.e.

$$
\sum_{i} \Omega_{i}+\Omega_{\kappa}=1
$$

Experimental constraints on the different parameters $\Omega_{i}$ and $\Omega_{\kappa}$ can be found in the latest Planck data release [69].

\footnotetext{
${ }^{2}$ The critical energy density $\rho_{\text {crit }}$ is the value required in order to have a spatially flat spacetime.
} 


\subsection{Motivations for inflation}

As pointed-out before, cosmic inflation provides one of the most successful scenario in order to solve the several issues of hot big bang cosmology. In the following we will briefly discuss these problems as a motivation for an inflationary phase in the primordial history of the universe.

\section{The Horizon problem}

In the hot big bang cosmology the primordial universe had a phase dominated by radiation. Then, the universe evolved to a non-relativistic matter scenario [70-72]. In both cases the Hubble constant was depending on time as

$$
a(t) \sim t^{\alpha} \Rightarrow H(t) \sim t^{-1}
$$

giving a negative acceleration $\ddot{a}(t)<0$. The particle horizon ${ }^{3}$ is set by the Hubble constant and increases with time as $\sim H^{-1} \sim t$.

In the big bang theory scenario, at the present time $t_{0}$, we should have causally access only to the regions of size of the order of $\sim t_{0}$. Therefore we expect that the universe is originated from various disconnected regions, since the causal size decreases going back in time. In particular we expect that for the cosmic microwave background, at the time of emission, during last-scattering, there are photons that are causally disconnected. The observed anisotropies in the cosmic microwave background appear to be the same in all directions, even for photons that have never been in causal contact.

This problem was solved by supposing a phase of rapid acceleration where all the scales are reduced. Therefore by supposing a sufficiently long inflationary epoch, the causal correlations in the cosmic microwave background are consistently explained.

In order to quantify the previous statement, one usually defines the number of e-folds

$$
N(t)=\int_{t}^{t_{\text {end }}} d \tau H(\tau)
$$

where $t_{\text {end }}$ is the time at the end of inflation. In order to solve the horizon problem a number of e-folds [73] $N>60$ is typically required.

\footnotetext{
${ }^{3}$ The particle horizon is the maximum distance from which particles could have reached the observer from the origin of the universe [71].
} 


\section{The Flatness problem}

The second problem that we would have without an inflationary phase arises from the observation that the universe seems to be flat at large scales. From the equation of the curvature one has

$$
\Omega(t)-1=\frac{\kappa}{H^{2}(t) a^{2}(t)},
$$

where $\Omega=\rho(t) / \rho_{\text {crit }}$. During the radiation and matter domination period, the value $\Omega-1$ increases in time. This means that in order to explain the flatness of the space observed today, the value of $\Omega-1$ should have started from a value extremely close to zero in the primordial universe.

Inflation provides an elegant solution to the flatness problem. Since the Hubble rate is almost constant during the inflationary phase, one has a deviation from $\Omega-1$ that is suppressed exponentially with time

$$
\Omega(t)-1 \propto \frac{1}{a^{2}(t)}
$$

In order to get a vanishing value of $\Omega(t)-1$ today, it is required that the inflationary epoch lasted for at least 70 e-folds [73]. It should be noted that the flatness problem is a fine-tuning problem. It is not a genuine inconsistency, but it requires a very unnatural value of $|\Omega-1| \sim 10^{-60}$ at the beginning of the radiation dominated era.

As pointed-out before, inflation provides an appealing scenario where the quantum fluctuations [74-76] of the primordial fields are streched to macroscopic scales and are responsible for the initial primordial curvature perturbations required by the hot big bang theory. The statistical properties of the initial perturbations can be directly related to late time observables as the temperature anisotropies in the cosmic microwave background or the observed large scale structure of our universe.

\subsection{Slow-roll inflation}

An inflationary period in the early universe is characterized by a positive acceleration of the scale factor $\ddot{a}>0$. From the Friedmann equations (2.6), this requires a negative pressure $p<-\frac{\rho}{3}$. In the following, the case of slow-roll inflation where $p \sim-\rho$ is discussed. An almost exponential expansion of the universe can be achieved by considering a single field model where the inflaton field is slowly rolling down a potential $V(\phi)$ that dominates over the canonical kinetic energy. Therefore an inflationary period 
can be obtained from a Lagrangian density

$$
\mathcal{L}=\sqrt{-g}\left[-\frac{1}{2} \partial_{\mu} \phi \partial^{\mu} \phi-V(\phi)\right],
$$

where $g$ is the determinant of the FRW metric. Restricting to the case of a perfect fluid, the pressure and the energy density for an homogeneous field are given by

$$
\rho=\frac{1}{2} \dot{\phi}^{2}+V(\phi), \quad p=\frac{1}{2} \dot{\phi}^{2}-V(\phi) .
$$

The equation of state becomes

$$
w=\frac{p}{\rho}=\frac{\frac{1}{2} \dot{\phi}^{2}-V(\phi)}{\frac{1}{2} \dot{\phi}^{2}+V(\phi)} .
$$

Therefore if the potential dominates over the kinetic term, the conditions for a de-Sitter universe are recovered and the scalar field becomes responsible for the accelerated expansion of the universe. The equation of motion for the inflaton field is described by the FRW equation

$$
\ddot{\phi}+3 H \dot{\phi}+V^{\prime}(\phi)=0
$$

and

$$
H^{2}=\frac{1}{3 M_{P}^{2}} \rho
$$

\section{Slow-roll parameters}

In the limit of slow-roll inflation the scalar field is slowly rolling down the potential with a subdominant kinetic energy. This is achieved by requiring that $\dot{\phi}^{2} \ll V(\phi)$. It is also expected, being the potential flat, that also $\dot{\phi}$ can be neglected. The FRW equation becomes

$$
H^{2}=\frac{1}{3 M_{P}^{2}} V(\phi)
$$

where it is assumed that the inflaton field is dominating the energy density of the universe. The equation of motion becomes

$$
\dot{\phi}=-\frac{V^{\prime}(\phi)}{3 H}
$$


The slow-roll conditions then require

$$
\dot{\phi}^{2} \ll V(\phi) \Rightarrow \frac{V^{\prime}(\phi)^{2}}{V(\phi)} \ll H^{2}
$$

and

$$
|\ddot{\phi}| \ll|3 H \dot{\phi}| \Rightarrow\left|V^{\prime \prime}(\phi)\right| \ll\left|H^{2}\right| .
$$

It can be useful to introduce the slow-roll parameters

$$
\begin{aligned}
\epsilon & =-\frac{\dot{H}}{H^{2}}=\frac{M_{P}^{2}}{2}\left(\frac{V^{\prime}(\phi)}{V(\phi)}\right)^{2}, \\
\eta & =M_{P}^{2}\left(\frac{V^{\prime \prime}(\phi)}{V(\phi)}\right), \\
\delta & =\eta-\epsilon .
\end{aligned}
$$

It should be noted that $\epsilon$ represents the slope of the potential and $\eta$ the curvature. An inflationary period is characterized by $\epsilon<1$ and slow-roll inflation by the condition that the potential is approximately constant, i.e. $|\eta|, \epsilon \ll 1$.

\subsection{Inflationary perturbations}

Because of the rapid expansion in the inflationary epoch, the early universe was nearly uniform. This very small deviation from homogeneity is the initial seed that explains our understanding of the origins of structures in the universe. This is one of the big success of inflation that was confirmed by the measurement of the temperature anisotropies in the cosmic microwave background. The following introduction is based on $[70-73]$.

The main idea of cosmological perturbation theory is to separate the fields $\chi(t, x)$ into a homogeneous part $\chi(t)$ that depends only on time and a small perturbation $\delta \chi(t, x):=\chi(t, x)-\chi(t)$ that depends also on the spatial coordinates. This approach is justified considering that at the time of decoupling the inhomogeneities were very small, of the order of $\delta \rho / \rho \sim 10^{-5}$.

In the following we consider how the perturbations of a generic scalar field $\chi$ behaves on a de-Sitter background. Let $\delta \chi_{k}$ be the Fourier transform with respect to the spatial coordinates of the field fluctuations $\delta \chi$.

The equation of motion in the case of a massless field reads

$$
\delta \ddot{\chi}_{k}+3 H \delta \dot{\chi}_{k}+\frac{k^{2}}{a^{2}} \delta \chi_{k}=0
$$


For scales within the Hubble radius $\lambda \ll H^{-1}$, the friction term $3 H \delta \dot{\chi}_{k}$ of eq. (2.32) can be neglected and the equation of motion reduces to the equation of an harmonic oscillator with a time-dependent frequency $k^{2} / a^{2}(t)$. Therefore for wavelengths smaller than the Hubble radius $H^{-1}$, the fluctuation is mostly oscillating. On the other hand for scales larger than the Hubble radius $\lambda \gg H^{-1}$ the term proportional to $k^{2} / a^{2}(t)$ can be neglected and a constant value $\left|\delta \chi_{k}\right|=H / \sqrt{2 k^{3}}$ becomes a solution to the equation of motion.

Eq. (2.32) has an exact solution given by

$$
\delta \chi_{k}=\frac{1}{a(t)}\left[C \frac{e^{-i k \tau}}{\sqrt{2 k}}\left(1-\frac{i}{k \tau}\right)+D \frac{e^{i k \tau}}{\sqrt{2 k}}\left(1+\frac{i}{k \tau}\right)\right],
$$

where $C, D$ are two constants that are determined by the initial conditions and $\tau$ is the conformal time. The constants $C$ and $D$ are fixed by imposing that at $\tau \rightarrow-\infty$ the mode $\delta \chi_{k}$ is given by the positive-frequency Minkowski solution $\delta \chi_{k} \propto e^{-i k \tau}$, i.e.

$$
\lim _{\tau \rightarrow-\infty} \delta \chi_{k}(\tau)=\frac{1}{a(t)} \frac{e^{-i k \tau}}{\sqrt{2 k}}
$$

which corresponds to the minimal excitation state. The initial condition sets the constants to $C=1$ and $D=0$. Eq. (2.33) reduces to

$$
\delta \chi_{k}=\frac{1}{a(t)} \frac{e^{-i k \tau}}{\sqrt{2 k}}\left(1-\frac{i}{k \tau}\right)
$$

which reproduces the previous qualitative considerations in the two different regimes, i.e. for $k \ll a H$ and for $k \gg a H$.

An important quantity that characterizes the properties of the perturbation is the power spectrum which is defined as

$$
(2 \pi)^{3} \delta^{(3)}\left(k+k^{\prime}\right) \mathcal{P}_{\delta \chi}(k)=\left\langle\delta \chi_{k} \delta \chi_{k^{\prime}}\right\rangle
$$

The power spectrum is directly connected to the Fourier modes $\delta \chi$ as

$$
\mathcal{P}_{\delta \chi}(k)=\left.\frac{k^{3}}{2 \pi^{2}}\left|\delta \chi_{k}\right|^{2}\right|_{k=a H}
$$

where the power spectrum is evaluated at horizon exit $k=a(t) H(t)$ giving an expression dependent only on the momentum variable $k$. After the horizon exit the spectrum of the field perturbations $\delta \chi$ is constant 


$$
\mathcal{P}_{\delta \chi}(k)=\frac{k^{3}}{2 \pi^{2}} \frac{H^{2}}{2 k^{3}}=\frac{H^{2}}{4 \pi^{2}}
$$

We discuss now the example of a massive scalar field with small mass $m_{\chi}$ in de-Sitter spacetime. The equation (2.32) becomes

$$
\delta \sigma_{k}^{\prime \prime}+\left(k^{2}+M^{2}(\tau)\right) \delta \sigma_{k}=0, \quad M^{2}(\tau)=\frac{1}{\tau^{2}}\left(\frac{m_{\chi}^{2}}{H^{2}}-2\right)
$$

where $M(\tau)$ is a time-dependent mass and $\tau$ is the conformal time. We also define

$$
\delta \sigma_{k}=a(t) \delta \chi_{k}
$$

in order to absorb the scale factor dependence into the field definition. By defining the index $\nu_{\chi}$ as

$$
\nu_{\chi}^{2}=\left(\frac{9}{4}-\frac{m_{\chi}^{2}}{H^{2}}\right)
$$

the massive equation of motion (2.39) reads then

$$
\delta \sigma_{k}^{\prime \prime}+\left[k^{2}-\frac{1}{\tau^{2}}\left(\nu_{\chi}^{2}-\frac{1}{4}\right)\right] \delta \sigma_{k}=0
$$

For a real index, the last equation has a solution given in terms of the Hankel's functions of the first and second kind $H_{\nu_{\chi}}^{(1)}$ and $H_{\nu_{\chi}}^{(2)}$ given by

$$
\delta \sigma_{k}=\sqrt{-\tau}\left(A(k) H_{\nu_{\chi}}^{(1)}(-k \tau)+B(k) H_{\nu_{\chi}}^{(2)}(-k \tau)\right),
$$

where $A$ and $B$ are two coefficients that have to be fixed with the initial conditions.

After imposing that in the ultraviolet regime, i.e. for scales $k \gg a H$, we recover the plane-wave solution $e^{-i k \tau} / \sqrt{2 k}$ and with the known limits [77]

$$
\begin{aligned}
& H_{\nu_{\chi}}^{(1)}(-k \tau \gg 1) \sim \sqrt{\frac{-2}{k \tau \pi}} e^{i\left(-k \tau-\frac{\pi}{2} \nu_{\chi}-\frac{\pi}{4}\right)}, \\
& H_{\nu_{\chi}}^{(2)}(-k \tau \gg 1) \sim \sqrt{\frac{-2}{k \tau \pi}} e^{-i\left(-k \tau-\frac{\pi}{2} \nu_{\chi}-\frac{\pi}{4}\right)},
\end{aligned}
$$

the constants are fixed to

$$
\begin{aligned}
& A(k)=\frac{\sqrt{\pi}}{2} e^{i\left(\nu_{\chi}+\frac{1}{2}\right) \frac{\pi}{2}}, \\
& B(k)=0 .
\end{aligned}
$$


The exact solution finally reads

$$
\delta \sigma_{k}=\frac{\sqrt{\pi}}{2} e^{i\left(\nu_{\chi}+\frac{1}{2}\right) \frac{\pi}{2}} \sqrt{-\tau} H_{\nu_{\chi}}^{(1)}(-k \tau)
$$

For scales larger than the horizon the Hankel's function $H_{\nu_{\chi}}^{(1)}$ is approximated to [77]

$$
H_{\nu_{\chi}}^{(1)}(-k \tau \ll 1) \sim \sqrt{\frac{2}{\pi}} e^{-i \frac{\pi}{2}} 2^{\left(\nu_{\chi}-\frac{3}{2}\right)}\left(\frac{\Gamma\left(\nu_{\chi}\right)}{\Gamma(3 / 2)}\right)(-k \tau)^{-\nu_{\chi}},
$$

where $\Gamma$ is the gamma function. Returning to the original cosmological perturbation $\delta \chi_{k}$, one finds that for scales larger than the horizon $-k \tau \ll 1$, the fluctuation of a scalar perturbation of a massive field is not constant but acquires a small dependence on time. Indeed

$$
\left|\delta \chi_{k}\right| \sim \frac{H}{\sqrt{2 k^{3}}}\left(\frac{k}{a H}\right)^{\frac{3}{2}-\nu_{\chi}}, \quad-k \tau \ll 1
$$

Before we considered the quantum fluctuations of a generic scalar field in a pure de-Sitter background where the expansion of the universe is described by the scale factor $a(\tau)=-1 /(k \tau)$. However, during the inflationary epoch the Hubble rate has a little change in time as $\dot{H}=-\epsilon H^{2}$ (quasi de-Sitter expansion), where $\epsilon$ is a small parameter. The scale factor becomes

$$
a(\tau)=-\frac{1}{H \tau^{1+\epsilon}}
$$

The scalar fluctuations are described by eq. (2.39) with the mass term now given by

$$
M^{2}(\tau)=m_{\chi}^{2} a^{2}(\tau)-\frac{a^{\prime \prime}(\tau)}{a(\tau)} \simeq m_{\chi}^{2} a^{2}(\tau)-\frac{1}{\tau^{2}}(2+3 \epsilon)
$$

where' denotes the derivative respect to the conformal time. Therefore for small values of $\epsilon$ and $\eta_{\chi} \equiv m_{\chi}^{2} /\left(3 H^{2}\right)$ we get eq. (2.42) with $\nu_{\chi}$ given by

$$
\nu_{\chi}^{\epsilon}=\frac{3}{2}+\epsilon-\eta_{\chi}
$$

The power spectrum evaluated at the horizon crossing reads now

$$
\mathcal{P}_{\delta \chi}(k)=\left(\frac{H}{2 \pi}\right)^{2}\left(\frac{k}{a H}\right)^{3-2 \nu_{\chi}^{\epsilon}}
$$


and one can define the spectral index of the perturbation as

$$
n_{\delta \chi}-1=\frac{d \ln \mathcal{P}_{\delta \chi}}{d \ln k}=3-2 \nu_{\chi}^{\epsilon}
$$

The tiny tilt in the power spectrum arises both because the Hubble rate is not constant during inflation and because the inflaton field was considered massive.

The previous considerations can be directly applied to the case of a scalar inflaton field. As discussed before, the inflaton is dominating the energy density of the universe in the inflationary stage and any perturbation of the field $\delta \phi$ leads to perturbation of the energy momentum tensor. Moreover, the Einstein's equations relate the perturbation of the stress energy-momentum tensor to perturbations of the metric

$$
\frac{1}{M_{P}^{2}} \delta T_{\mu \nu}=\delta G_{\mu \nu}
$$

This chain is closed by noticing that a perturbation of the metric changes the KleinGordon equation of the inflaton field giving back the inflaton perturbations. This procedure suffers from the complication of the backreaction effect. Moreover the splitting into background and fluctuations depends on the choice of coordinates.

In order to avoid problems related to the gauge dependence of the results, one typically introduces gauge independent quantities from the matter and metric perturbations [78]. The first gauge-invariant quantity is measuring the spatial curvature of hypersurfaces with a constant energy density and reads [79]

$$
-\zeta:=\Psi+\frac{H}{\dot{\rho}} \delta \rho
$$

where $\Psi$ is the curvature perturbation on a generic slicing and $\rho$ are the density perturbations.

During inflation the quantity $\zeta$ remains constant after the horizon exit (for $k \ll a H$ ). During slow-roll, eq. (2.55) simplifies to

$$
-\zeta=\Psi+\frac{H}{\dot{\phi}} \delta \phi
$$

With a proper choice of gauge that has spatially flat hypersurface, the gauge-invariant parameter $\zeta$ can be connected to late time observables through

$$
\zeta \sim \frac{\delta \rho}{\rho+p}
$$

The second gauge-invariant quantity which is used in the literature is the comoving 
curvature perturbation

$$
\mathcal{R}:=\frac{H}{\dot{\phi}} \delta \phi,
$$

and by construction it represents the gravitational potential on comoving hypersurfaces, i.e. hypersurfaces with $\delta \phi=0$. Any linear combination of $\mathcal{R}$ and $\zeta$ will preserve the gauge-invariance. Moreover, in slow-roll inflation, on super-Hubble scales one has $\zeta \sim \mathcal{R}$

We conclude this section by relating the power spectrum of the curvature fluctuations $\mathcal{R}_{k}$ to the power spectrum of the inflaton fluctuations $\delta \phi_{k}$. The longitudinal gauge is a convenient choice in order to compute the cosmological perturbations because one has

$$
\mathcal{R}_{k} \simeq H \frac{\delta \phi_{k}}{\dot{\phi}}
$$

The power spectrum of the comoving curvature perturbation after the horizon exit reads then

$$
\mathcal{P}_{\mathcal{R}}=\frac{k^{3}}{2 \pi^{2}} \frac{H^{2}}{\dot{\phi}^{2}}\left|\delta \phi_{k}\right|^{2} .
$$

If the power spectrum of the inflaton fluctuations $\delta \phi$ is described by eq. (2.52), one finally has

$$
\mathcal{P}_{\mathcal{R}}=\frac{H^{2}}{\dot{\phi}^{2}}\left(\frac{H}{2 \pi}\right)^{2}\left(\frac{k}{a H}\right)^{3-2 \nu_{\chi}^{\epsilon}} .
$$

\subsection{Selection of classes of models}

Inflationary models are classified according to their properties. In the following three different categories are considered: large-field, small-field, and hybrid models. Generically, a single-field potential is described by

$$
V(\phi)=\Lambda^{4} f\left(\frac{\phi}{\mu}\right),
$$

where the amplitude $\Lambda^{4}$ corresponds to the vacuum energy density and the width $\mu$ is related to the field's change during the inflationary phase. Different inflationary models consider different functions $f$. In Figure 2.1 we show an example of a slow-roll single-fields model. 


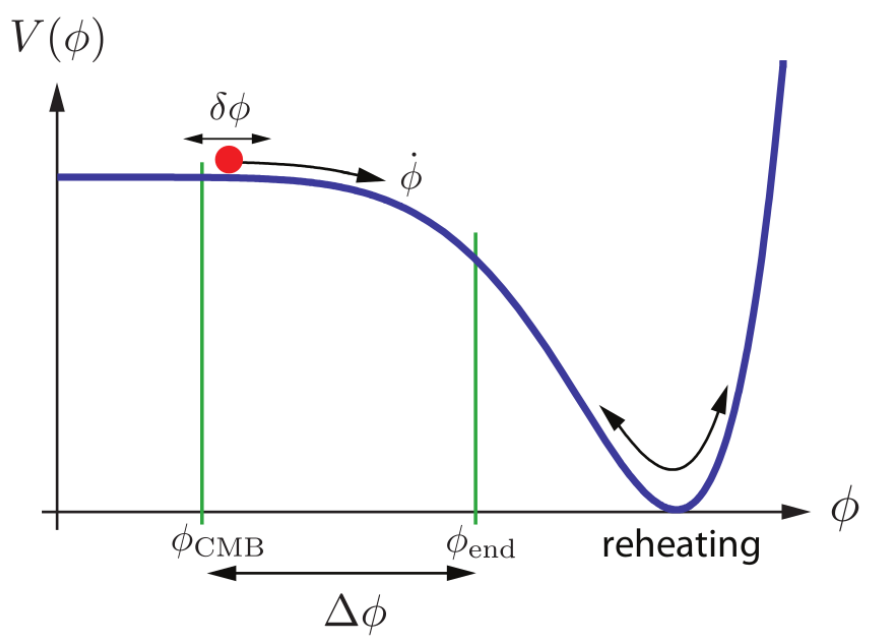

Figure 2.1: Example of a slow-roll single-field inflation. The accelerating expansion of the universe occurs when the potential $V(\phi)$ is dominating over the canonical kinetic energy $\frac{1}{2} \dot{\phi}^{2}$. Inflation ends at $\phi_{\text {end }}$ when the kinetic term becomes comparable to the inflaton potential. The cosmic microwave background fluctuations are generated about 60 e-folds before the end of inflation from quantum fluctuations. The Figure was taken from $[80]$.

\section{Large-field models}

Large-field inflation is an interesting class of models where the prototype potential is given by the chaotic scenario [40] of a monomial interaction, i.e.

$$
V(\phi)=\lambda\left(\frac{\phi}{\mu}\right)^{n}
$$

For these models, the scalar field is displaced from the minimum of the inflaton potential by an amount of the order of the Planck mass. These models are characterized by potentials with positive curvature, i.e. $V^{\prime \prime}(\phi)>0$. The large potential $V(\phi) \sim M_{P}^{4}$ results in a large friction in the Friedmann equation with the consequence that the inflaton slowly rolls down the potential. An important feature of the chaotic scenario is that the slow-roll conditions are independent from the coupling $\lambda$ and that they produce an amount of gravitational waves that potentially can be observed in the future. The simplest model is given by a quadratic potential

$$
V(\phi)=\frac{1}{2} m^{2} \phi^{2}
$$

where $m$ represents the mass of the inflaton. Inflation ends when the field's magnitude becomes comparable to the Planck's mass, because the slow-roll conditions are violated. 


\section{Small-field models}

Small-field models are in general obtained from the spontaneous symmetry breaking and inflation starts from near an unstable vacuum. The field is rolling down the potential to the stable minimum. Small-field scenarios typically have a negative curvature of the potential, i.e. $V^{\prime \prime}(\phi)<0$ and $\epsilon$ is very close to zero. A simple example is given by the Higgs-type potential

$$
V(\phi)=V_{0}\left[1-\left(\frac{\phi}{\mu}\right)^{2}\right]^{2},
$$

that can be interpreted as the lowest-order expansion of generic potentials. These models predict negligible production of tensor perturbations [81].

An interesting model is given by natural inflation [82] described by the potential

$$
V(\phi)=V_{0}\left[\cos \left(\frac{\phi}{f}\right)+1\right]
$$

where the inflaton field could be identified with an axion. Depending on the value of $f$ this could be a small-field or a large-field model.

\section{Hybrid models}

A richer phenomenology is given by multi-field inflation [83], where models with more than one field contribute to the inflationary dynamics. On the other hand, this will also increase the number of parameters of the theory with the consequent loss of predictive power. This class is popular for models inspired by supersymmetry and supergravity since they provide the additional fields required by the hybrid inflation.

Here we consider only the hybrid model where the inflaton field $\phi$ evolves toward a minimum of the potential with non-zero energy. The end of inflation is determined from a second field $\sigma$ that act as a clock of the inflationary phase. These models have a positive curvature in the inflaton field direction. An example of a hybrid theory is given by the following potential

$$
V(\phi, \sigma)=\frac{m^{2}}{2} \phi^{2}+\frac{1}{4 g}\left(M^{2}-g \sigma^{2}\right)^{2}+\lambda_{h}^{2} \phi^{2} \sigma^{2}
$$

For an inflaton field $\phi$ larger than the critical value $\phi_{c}=M /\left(\sqrt{2} \lambda_{h}\right)$ the only minimum is at $\sigma=0$. Therefore at the beginning of inflation the field $\sigma$ is rolling down the potential to $\sigma=0$ while $\phi$ remains large and drives inflation. Inflation ends when $\phi$ becomes smaller than $M /\left(\sqrt{2} \lambda_{h}\right)$. Then a phase transition occurs and the two fields $\phi$ and $\sigma$ rapidly fall into the absolute minimum of the potential at $\phi=0$ and $\sigma^{2}=M^{2} / g$. 


\section{CHAPTER 3}

\section{—QUANTUM FIELD THEORY IN CURVED SPACETIME}

Quantum field theory ${ }^{1}$ (QFT) is one of the most successful theory of modern physics and it combines quantum mechanics with special relativity. Quantum mechanics is a non relativistic theory and cannot consistently describe processes where the number and the type of particles change, as in the case in most reactions in nuclear and particle physics. Moreover, any attempt to construct relativistic wave equations had the problem that negative energy solutions were appearing without a clear interpretation. Quantum field theory gave a different theoretical framework where these difficulties could be solved. In the new point of view, particles are identified as the field's modes and the interactions between particles are described by the interaction of the corresponding underlying fields. It is also a universal language used in many fields of science and it gives a consistent description of collective phenomena. Unfortunately one finds some technical difficulties in the attempt to describe gravity with a complete quantum field theory because it becomes non-renormalizable.

In Section 3.1 we will introduce the problem of non-renormalizability and motivate the use of a semi-classical approach where gravity is described as a classical background. Then, in Section 3.2 we will review the standard renormalization techniques in quantum field theory in order to avoid the nonphysical divergences that arise naturally from the formalism. In Section 3.3, the adiabatic renormalization is discussed for quantum field theories on a FRW background. The formal approach to renormalization is also presented in Section 3.4. The perturbative approach within the closed-time-path (CTP) formalism is finally explored in Chapter 4.

\footnotetext{
${ }^{1}$ See $[84,85]$ for a general review and [86] for a recent introduction to quantum field theory.
} 


\subsection{Introduction}

In the classical theory of general relativity the Einstein's equations [58-62] can be derived from the Einstein-Hilbert action

$$
S=\frac{M_{P}^{2}}{2} \int d^{4} x \sqrt{-g}(\mathcal{R}-2 \Lambda)
$$

where $M_{P}$ is the reduced Planck mass, $g$ is the determinant of the metric, $\mathcal{R}$ is the scalar Ricci curvature, and $\Lambda$ is the cosmological constant. After imposing a null variation of the action, the Einstein's equations in vacuum are found, i.e.

$$
R_{\mu \nu}-\frac{1}{2} g_{\mu \nu} \mathcal{R}+\Lambda g_{\mu \nu}=0
$$

This equation is generalized by adding into the action a term that takes into account the matter content of the system. One finds

$$
R_{\mu \nu}-\frac{1}{2} g_{\mu \nu} \mathcal{R}+\Lambda g_{\mu \nu}=\frac{1}{M_{P}^{2}} T_{\mu \nu}
$$

where $T_{\mu \nu}$ is the energy momentum tensor associated to the matter component.

Here the metric $g_{\mu \nu}$ plays a twofold role. On one side, it describes the physical background of the theory. On the other side it is dynamical as one can see in eq. (3.3). When one tries to consider $g_{\mu \nu}$ as a field whose dynamics is described from an action principle, the theory becomes non-renormalizable.

A possible way around is to consider the semi-classical approach of quantum field theory, where one considers quantum fields in a classical background and gravity enters into the theory as an overall factor $\sqrt{-g}$ in front of the Lagrangian density. By imagining the metric given by a perturbative expansion

$$
g_{\mu \nu}=g_{\mu \nu}^{(0)}+\hbar g_{\mu \nu}^{(1)}+\mathcal{O}\left(\hbar^{2}\right)
$$

the semi-classical approach consists of the zeroth order in $\hbar$. This is the theoretical framework that will be used in this work.

Other solutions are of course possible. For example, one can forget about the nonrenormalizability of the quantum field theory of gravity and consider an effective theory with higher graviton loops. Another solution is to treat the background classically and to consider the back-reaction from the Einstein's equation in the following way

$$
R_{\mu \nu}-\frac{1}{2} g_{\mu \nu} \mathcal{R}+\Lambda g_{\mu \nu}=\frac{1}{M_{P}^{2}}\left\langle T_{\mu \nu}\right\rangle_{\phi}
$$


where the expectation value of stress energy-momentum tensor is quantized in some state $\phi$.

Moreover, the Lorentz invariance plays a fundamental role in quantum field theory in a flat spacetime. Since in this case one can identify a unique vacuum state for the theory, one has a well-defined procedure in order to construct and interpret quantum states and physical observables. In an arbitrary spacetime, where the Poincaré invariance is broken, there is in general no unique definition for a vacuum state. Therefore notions like particles or the scattering amplitude become ambiguous. For a general review about quantum field theory in curved spacetime see [37, 87-90].

In Minkowski spacetime, after the second canonical quantization we typically find the following Fourier expansion of the field [87]

$$
\phi(x)=\sum_{i}\left(a_{i} u_{i}+a_{i}^{\dagger} u_{i}^{*}\right)
$$

where $u_{i}$ are the field's modes and $a_{i}^{\dagger}, a_{i}$ the creation and annihilation operators. This expression gives a unique definition of the vacuum state $|0\rangle$ as the state such that $a_{i}|0\rangle=0$ for all $i$. In a curved spacetime the situation changes. Since there is no unique choice for the field's modes $u_{i}$, the vacuum is not unique. As a consequence one cannot describe the particle content of a state because there is no notion of vacuum, except for asymptotic states where it is supposed that at infinity the curvature vanishes.

The non-uniqueness of the vacuum gives rise to physically interesting scenarios of particle creation in cosmological and black holes spacetimes that are reviewed in [91, 92]. One example is given by the Hawking effect [93-95], where black holes emit a thermal spectrum of radiation. The creation of particles is an important feature in order to be consistent with the second law of thermodynamics. Another important phenomenon is given by the Unruh effect [96] that predicts that an accelerating observer detects a black body radiation, not seen by the inertial observer.

In this thesis we are mostly interested in scalar field theories in a de-Sitter spacetime. In this particular scenario the vacuum state is typically defined by requiring that the high frequency asymptotic form is given by the Minkowski vacuum and by requiring also de-Sitter invariance. This gives a preferred set of modes [80]

$$
u_{k}=\frac{e^{-i k \tau}}{\sqrt{2 k}}\left(1-\frac{i}{k \tau}\right) \text {. }
$$

This state is called the de-Sitter invariant vacuum or the Bunch-Davies vacuum [20] and is the most natural vacuum state in order to calculate the explicit form of the propagators and expectation values in a FRW spacetime. 


\subsection{Renormalization in Minkowski spacetime}

In quantum field theory, renormalization $[84,85]$ is the collection of techniques used in order to deal with infinities that arise in the perturbative calculation of physical quantities. For example, for a scalar field theory with interaction $\lambda \phi^{4}$, the following integral appears in the first order perturbative expansion of the $2 \rightarrow 2$ scattering amplitude [86]

$$
I(p)=\frac{(-i \lambda)^{2}}{2} \int \frac{d^{4} k}{(2 \pi)^{4}} \frac{i}{k^{2}-m^{2}+i \epsilon} \frac{i}{(p-k)^{2}-m^{2}-i \epsilon},
$$

where $\epsilon \rightarrow 0^{+}, p$ represents the external momenta, and $m$ the mass of the field. This integral is related to a scattering amplitude that can be measured and therefore it should be finite. In order to understand the general ideas of renormalization let's discuss how to extract a meaningful quantity from $I(0)$. The first step is to regularize the theory by making the integral (3.8) finite. For example, we can introduce a cutoff ${ }^{2}$ $\Lambda$ in the momentum integral, i.e.

$$
\begin{aligned}
{[I(0)]_{\mathrm{reg}} } & =\frac{i \lambda^{2}}{2} \frac{1}{(2 \pi)^{4}}\left(2 \pi^{2}\right) \int^{\Lambda} \frac{d k}{k}+\text { finite contributions } \\
& =i \lambda^{2} \frac{1}{16 \pi^{2}} \log \Lambda+\text { finite contributions }
\end{aligned}
$$

where the factor $\left(2 \pi^{2}\right)$ is due to the solid angle integration. In the previous expression the divergence was isolated to a logarithmic term. This term can be subtracted from eq. (3.9) and what remains is a meaningful quantity that can be confronted to the observations and that is predictive. This is the beauty of renormalization. The formal approach of renormalization in the language of distribution theory will be addressed in Section 3.4.

In the previous case, with the prescription ${ }^{3}$ where only the divergent contributions are removed, the renormalized $I(0)$ reads

$$
[I(0)]_{\mathrm{ren}}=\text { finite contributions. }
$$

Another example is given by the one-loop correction to the two-point function, which is represented by a tadpole diagram. The integral is of the form of

$$
\frac{-i \lambda}{2} \int \frac{d^{4} k}{(2 \pi)^{4}} \frac{1}{k^{2}+m^{2}}
$$

\footnotetext{
${ }^{2}$ There are other convenient choices of regularization schemes as dimensional regularization [84].

${ }^{3}$ This prescription is called the minimal subtraction scheme.
} 
After the introduction of a cutoff the regulated tadpole reads

$$
-i \frac{\lambda}{32 \pi^{2}}\left(\Lambda^{2}-m^{2} \log \left(\frac{\Lambda^{2}+m^{2}}{m^{2}}\right)\right) .
$$

This integral has quadratic and logarithmic divergences, both arising in the ultraviolet regime. This and similar divergences are found in the perturbative calculation of higher order correlation functions and can be absorbed in the definition of physical quantities in the Lagrangian. For example for a theory with quartic interaction considered before one can define counter-terms $\delta \mathcal{L}$ in the Lagrangian that depend on the cutoff $\Lambda$ of the regulated theory

$$
\mathcal{L}[\phi]=\left(\frac{1}{2} \partial_{\mu} \phi \partial^{\mu} \phi-\frac{1}{2} m^{2} \phi^{2}-\frac{\lambda}{4 !} \phi^{4}\right)+\delta \mathcal{L}
$$

where

$$
\delta \mathcal{L}=\left(\frac{1}{2} \delta Z \partial_{\mu} \phi \partial^{\mu} \phi-\frac{1}{2} \delta m^{2} \phi^{2}-\frac{\delta \lambda}{4 !} \phi^{4}\right)
$$

As a consequence of this procedure, the renormalized coupling constants depend on the energy scale of the measurement. In this thesis we will mainly work in the minimal subtraction scheme (MS) $[97,98]$ where the counter-terms are defined in order to absorb only the divergent terms of the radiative corrections.

With the previous examples we saw that by introducing the counter-terms in eq. (3.13) one is able to cure all the divergences of the two- and four-point correlation function. In principle we could continue and investigate loop diagrams appearing for the six-point function and so on. It can be proven [85] that for the scalar field theory (3.13), all the divergences can be absorbed by a redefinition of the counter-terms given in eq. (3.14). In this case the theory is called renormalizable. If this were not the case and genuinely new divergences appear in the calculation of higher order correlation functions, we should introduce new counter-terms in the Lagrangian (e.g. a term proportional to $\left.\phi^{6}\right)$. If this process never stops and for each higher order amplitude one has to introduce new counter-terms, the theory is said to be non-renormalizable. This class of theories are less predictive, because one has to introduce an infinite amount of parameters that have to be fixed by experiments. Nevertheless non-renormalizable theories are still predictive at low-energy because the higher order corrections can be neglected.

In the next section, we will introduce the adiabatic regularization, which is a prescription used for calculating finite expectation values that involve quadratic products of fields in a FRW background. The other example of renormalization in curved spacetime is given by renormalization in the CTP formalism, that allows 
to use the renormalization techniques developed in Minkowski spacetime also for time-dependent backgrounds. The formalism is discussed in Chapter 4 .

\subsection{Adiabatic renormalization}

In the following the adiabatic expansion procedure $[92,99]$ in a Friedmann Lemaitre Robertson Walker universe is discussed. The metric is assumed to be of the form

$$
d s^{2}=d t^{2}-a^{2}(t) d x^{2}
$$

where $(t, x) \in \mathbb{R}^{4}$ are the usual comoving coordinates and $a(t)$ is the scale factor which takes into account the expansion of the universe.

This explicit time dependence in the scale factor breaks the time-translation invariance of the system, therefore the first question that has to be addressed is how to construct a Hilbert space when the system has less symmetries. We mainly have two conditions, first one requires that our chosen basis can reproduce all the physical expectation values that one can construct. Secondly, one expects that particles are not created in the limit where the single-particle energy is larger compared to the scale of energy of the curvature. In other words, the number of particles has to remain almost invariant for a varying $a(t)$. This condition guarantees that quantities like the number of particles or the energy-momentum tensor give the expected values in the limit of small curvature. The asymptotic condition is often called the adiabatic condition.

It is useful to introduce a dimensionless parameter $T$ in order to quantify the slowness of the scale factor, i.e. $a(t) \rightarrow a(t / T)$. If we expand $a(t / T)$ around $T=\infty$ one has

$$
a(t / T)=a(0)+\frac{t}{T} a^{\prime}(0)+\frac{t^{2}}{2 T^{2}} a^{\prime \prime}(0)+\mathcal{O}\left(T^{-3}\right) .
$$

The zeroth-order of the adiabatic expansion gives precisely a flat and static universe. Usually in the literature [100], the power of $T^{-1}$ is called the adiabatic order. We remark that the number of derivatives in the expansion are directly correlated with the adiabatic order.

In order to construct the Hilbert space of the system, we start from the equation of motion

$$
\left(\square-M^{2}(t)\right) \phi=0,
$$

where $M$ is a time-dependent mass. The differential equation can be solved by introducing a set of complete orthonormal functions $u_{k}$ that satisfy the adiabatic condition. 
A scalar field can be expressed in terms of creation and annihilation operators $a_{k}^{\dagger}, a_{k}$

$$
\phi(x)=\int d k^{n-1}\left(a_{k} u_{k}(x)+a_{k}^{\dagger} u_{k}^{*}(x)\right)
$$

Since the choice of the basis of the Hilbert space is not unique, the adiabatic vacuum (and consequently the Fock space) will depend on the chosen $u_{k}$. Even if the Fock space is not unique, this representation can be used to describe physical events because it will uniquely determine the large momenta behavior.

Let's start with an Ansatz for the fields modes $u_{k}$

$$
u_{k}(x)=\frac{1}{\sqrt{2(2 \pi)^{n-1} a(t)^{n-1}}} h_{k}(t) e^{i k x},
$$

where the system is supposed to be on a n-dimensional spacetime for further convenience and

$$
h_{k}(t)=\frac{1}{\sqrt{W}} e^{-i \int^{t} d t^{\prime} W} .
$$

Assuming that our spacetime is described by a FRW-type metric, one can expand $W$ adiabatically

$$
W=c_{0}+c_{1} \frac{\dot{a}}{a}+c_{2} \frac{\dot{M}}{M}+c_{3} \frac{\dot{a}^{2}}{a^{2}}+c_{4} \frac{\dot{M}^{2}}{M^{2}}+c_{5} \frac{\ddot{a}}{a}+c_{6} \frac{\ddot{M}}{M}+c_{7} \frac{\dot{a} \dot{M}}{a M}+\mathcal{O}\left(T^{-3}\right),
$$

where $c_{i}$ are functions of the scale factor $a(t)$ and the effective mass $M(t)$. We define now the A-th order of $u_{k}$ as in eq. (3.19), where we have considered $W$ up to adiabatic order $A$. The approximated modes are denoted as $u_{k}^{(A)}$ and they are explicitly computed in $[34,35,101]$. This choice of modes also defines the creation and annihilation operators and the adiabatic vacuum $\left|0^{(A)}\right\rangle$. Therefore one has

$$
\phi(x)=\int d k^{n-1}\left(a_{k}^{(A)} u_{k}^{(A)}+a_{k}^{\dagger(A)} u_{k}^{*(A)}\right) .
$$

The adiabatic expansion will be applied to the energy-momentum tensor in order to have a theoretical framework where renormalization can be performed. First of all, the action is defined as [34]

$$
S\left[\varphi, g^{\mu \nu}\right]=S_{m}\left[\varphi, g^{\mu \nu}\right]+S_{g}\left[g^{\mu \nu}\right]
$$


where $g_{\mu \nu}$ is the metric and $S_{m}, S_{g}$ are the matter and gravitational part, i.e.

$$
\begin{aligned}
S_{m}\left[\varphi, g^{\mu \nu}\right] & =-\frac{1}{2} \int d^{n} x \sqrt{-g}\left[\partial_{\mu} \varphi \partial^{\mu} \varphi+m^{2} \varphi^{2}+\xi \mathcal{R} \varphi^{2}+2 \frac{\lambda}{4 !} \varphi^{4}\right], \\
S_{g}\left[g^{\mu \nu}\right] & =\int d^{n} x \sqrt{-g}\left[\Lambda+\alpha \mathcal{R}+\beta \mathcal{R}^{2}+\epsilon_{1} R_{\alpha \beta} R^{\alpha \beta}+\epsilon_{2} R_{\alpha \beta \gamma \delta} R^{\alpha \beta \gamma \delta}\right],
\end{aligned}
$$

where $R_{\mu \nu}$ and $\mathcal{R}$ are the Ricci tensor and scalar, $R_{\mu \nu \rho \sigma}$ is the Riemann curvature tensor, $\Lambda$ is the cosmological constant and $g$ is the determinant of the metric $g_{\mu \nu}$.

The energy-momentum tensor is obtained taking the variation of the action with respect to the metric

$$
T_{\mu \nu}=-\frac{2}{\sqrt{-g}} \frac{\delta S_{m}\left[\varphi, g^{\mu \nu}\right]}{\delta g^{\mu \nu}} .
$$

In the following the adiabatic regularization methods are discussed and applied to the case of the energy-momentum tensor $T_{\mu \nu}$.

\section{Adiabatic regularization}

Adiabatic subtraction [100, 102-105] is considered one of the most efficient methods for calculating finite expectation values that involve quadratic product of fields ${ }^{4}$.

It can be proven that the ultraviolet behavior of a quantity is contained in the lowest orders of its adiabatic expansion, or, equivalently that higher orders are less divergent. Consequently, one can define a regularization and renormalization procedure called adiabatic subtraction where the finite part is obtained by taking the expectation value of the full energy-momentum tensor $\left\langle T_{\mu \nu}\right\rangle$, containing the ultraviolet divergences and by subtracting all the divergent adiabatic orders ${ }^{5}$, i.e.

$$
\left\langle T_{\mu \nu}\right\rangle_{\text {finite }}=\left\langle T_{\mu \nu}\right\rangle-\left\langle T_{\mu \nu}\right\rangle^{(0)}-\left\langle T_{\mu \nu}\right\rangle^{(2)}-\left\langle T_{\mu \nu}\right\rangle^{(4)}
$$

The previous method has the advantage that it simultaneously regulates and renormalizes quantities. On the other hand, one might want to know the explicit form of the infinities and give an explicit expression for the counter-terms.

An alternative procedure was introduced in [34, 35, 106], where they first used dimensional regularization in order to make all the quantities finite. Thus, renormalization is applied only to the first adiabatic orders that contain all the divergences. In the following, the last method will be applied to the energy-momentum tensor.

In order to get the one-loop corrections to the energy-momentum tensor, it is

\footnotetext{
${ }^{4}$ For example $\left\langle\phi(x)^{2}\right\rangle$ or $\left\langle T^{\mu \nu}(x)\right\rangle$.

${ }^{5}$ For the energy-momentum tensor it can be shown that the ultraviolet behavior is contained in the adiabatic expansion up to order 4 .
} 
convenient to expand $\varphi$, that from now will be denoted as $\hat{\varphi}$, about the classical background $\varphi=\langle\hat{\varphi}\rangle$

$$
\hat{\varphi}=\varphi+\hat{\phi}
$$

where $\hat{\phi}$ is supposed to be Gaussian ${ }^{6}$ at one-loop order. By replacing eq. (3.28) in (3.24) and considering terms up to quadratic order, one obtains the explicit expression for the energy-momentum tensor at one-loop order [34]

$$
\begin{aligned}
T_{\mu \nu} & =-\frac{g_{\mu} \nu}{2}\left[\partial_{\rho} \varphi \partial^{\rho} \varphi+m^{2} \varphi^{2}+2 \frac{\lambda}{4 !} \varphi^{4}\right]+\partial_{\mu} \varphi \partial_{\nu} \varphi+\xi\left[G_{\mu \nu}-\nabla_{\mu} \nabla_{\nu}+g_{\mu \nu} \square\right] \varphi^{2} \\
& -\frac{g_{\mu} \nu}{2}\left[\partial_{\rho} \hat{\phi} \partial^{\rho} \hat{\phi}+m^{2} \hat{\phi}^{2}+\frac{\lambda}{2} \hat{\phi}^{2} \varphi^{2}\right]+\partial_{\mu} \hat{\phi} \partial_{\nu} \hat{\phi}+\xi\left[G_{\mu \nu}-\nabla_{\mu} \nabla_{\nu}+g_{\mu \nu} \square\right] \hat{\phi}^{2} \\
& =T_{\mu \nu}^{C}+T_{\mu \nu}^{Q} .
\end{aligned}
$$

In order to have the renormalized expression for the energy-momentum tensor, the following counter-terms $\delta T_{\mu \nu}$ are introduced

$$
T_{\mu \nu}=T_{\mu \nu}^{C}+T_{\mu \nu}^{Q}+\delta T_{\mu \nu}
$$

where

$$
\delta T_{\mu \nu}=-\frac{g_{\mu} \nu}{2}\left[\delta m^{2} \varphi^{2}+2 \frac{\delta \lambda}{4 !} \varphi^{4}\right]+\delta \xi\left[G_{\mu \nu}-\nabla_{\mu} \nabla_{\nu}+g_{\mu \nu} \square\right] \varphi^{2}-\delta T_{\mu \nu}^{g}
$$

and

$$
\delta T_{\mu \nu}^{g}=-g_{\mu \nu} \delta \Lambda+2 \delta \alpha G_{\mu \nu}+2 \delta \beta^{(1)} H_{\mu \nu}+2 \delta \epsilon_{1}{ }^{(2)} H_{\mu \nu}+2 \delta \epsilon_{2} H_{\mu \nu}
$$

The $H$ functionals represent the quadratic order tensors in the curvature and they are defined as

$$
\begin{aligned}
{ }^{(1)} H_{\mu \nu} & :=\frac{1}{\sqrt{-g}} \frac{\delta}{\delta g^{\mu \nu}} \int d^{n} x \sqrt{-g} \mathcal{R}^{2} \\
{ }^{(2)} H_{\mu \nu} & :=\frac{1}{\sqrt{-g}} \frac{\delta}{\delta g^{\mu \nu}} \int d^{n} x \sqrt{-g} R^{\mu \nu} R_{\mu \nu} \\
H_{\mu \nu} & :=\frac{1}{\sqrt{-g}} \frac{\delta}{\delta g^{\mu \nu}} \int d^{n} x \sqrt{-g} R^{\mu \nu \sigma \rho} R_{\mu \nu \sigma \rho} .
\end{aligned}
$$

\footnotetext{
${ }^{6}$ This implies that at one-loop order all $\left\langle\hat{\phi}^{k}\right\rangle$ vanishes for odd $\mathrm{k}$.
} 
By replacing the quantum field $\hat{\phi}$ with the Ansatz given in eq. (3.22), one has

$$
\left\langle T_{00}^{Q}\right\rangle=\int d^{n-1} k\left\{\frac{1}{2}\left[\left|\dot{u}_{k}\right|^{2}+\left(k^{2} / a^{2}+M^{2}\right)\left|u_{k}\right|^{2}\right]+\xi\left[G_{00}+(n-1) \frac{\dot{a}}{a} \partial_{0}\right]\left|u_{k}\right|^{2}\right\},
$$

where $M^{2}=m^{2}+\lambda / 2 \varphi^{2}$. The adiabatic expansion procedure can be applied in order to regularize the 00-component of the energy-momentum tensor. First, the operator $T_{00}^{Q}$ can be expanded adiabatically up to order 4 . The other terms are neglected since they do not contribute to the ultraviolet behavior

$$
\left\langle T_{00}^{Q}\right\rangle=\left\langle T_{00}^{Q}\right\rangle^{(0)}+\left\langle T_{00}^{Q}\right\rangle^{(2)}+\left\langle T_{00}^{Q}\right\rangle^{(4)}
$$

Finally $T_{00}$ is renormalized using dimensional regularization ${ }^{7}$ with the well-known procedure. The explicit calculation are performed in Section 5.4, where the energymomentum tensor is renormalized in the case where the renormalization conditions are given in Minkowski and de-Sitter spacetime. This gives an independent calculation of counter-terms that can be compared with alternative regularization prescriptions in curved space-time. In the next section the issue how to formally approach the problem of renormalization is addressed.

\subsection{From distribution theory to the Epstein-Glaser renormalization}

This chapter introduces the basic concepts regarding distribution theory. In particular, the question how to define the singular structure of distributions is approached. The issue how to consistently extend the domain of definition for supports that include the singularities is known as the Epstein-Glaser renormalization [107] and it is related to the renormalization procedure used in particle physics.

In the first part of this section the basic definitions of distribution theory are shown, including the definition of singular support. Then, the notions of renormalization of distribution and renormalization freedom are presented. This section concludes with a discussion of the wavefront set and of the singular structure of the different propagators.

\section{Distribution theory}

In physics one has often to deal with objects that have similar properties to functions despite they are not. The typical example is the delta function $\delta$, that is sometimes

\footnotetext{
${ }^{7}$ See Appendix C for more details or [160] for the original reference.
} 
(wrongly) defined as the real function $\delta: \mathbb{R} \rightarrow \mathbb{R}$ such that

$$
\delta(x)=\left\{\begin{array}{ll}
\infty, & x=0 \\
0, & \text { otherwise }
\end{array},\right.
$$

with $\int d x \delta(x)=1$. It can be proven that no functions can reproduce these two properties. Nevertheless, the previous definition of $\delta$ can be used in order to get meaningful results. As a representative example, $m \delta(x)$ corresponds to the physical description of the mass distribution of an ideal particle of mass $m$. Since the particle is point-like, all the mass is concentrated at $x=0$ and the total mass is given by

$$
\int_{\mathbb{R}} d x m \delta(x)=m \int_{\mathbb{R}} d x \delta(x)=m .
$$

Similar objects appear also as solutions of partial differential equations or as fundamental objects in QFT. In the following the basic definitions about distribution theory are introduced (for a general review see [108]). In order to simplify the notation, they are given for subregions of $\mathbb{R}^{n}$. Let $\mathcal{U} \subset \mathbb{R}^{n}$ be a non-empty open set.

Definition 3.4.1 (Test functions).

The space $\mathcal{D}(\mathcal{U})$ is defined as the space of smooth, compactly supported functions, also denoted as $\mathcal{D}(\mathcal{U})=C_{0}^{\infty}(\mathcal{U})$. A function $f \in \mathcal{D}(\mathcal{U})$ is called test function.

To introduce the notion of convergence on this space, we equip $\mathcal{D}$ with the topological structure of a Fréchet space, defined by the semi-norms [109]

$$
p_{\alpha, K}(f)=\sup _{x \in K}\left|\partial^{\alpha} f(x)\right|
$$

where $K \subset \mathcal{U}$ is a compact set and $\alpha$ is a multiindex. Therefore, a sequence of functions $f_{n} \in \mathcal{D}$ converges to $f \in \mathcal{D}$ if and only if $p_{\alpha, K}\left(f_{n}-f\right) \rightarrow 0$ as $n \rightarrow \infty$ for all $\alpha$ on compact subsets. Distributions are defined as the space of continuous linear functionals on $\mathcal{D}(\mathcal{U})$.

Definition 3.4.2 (Space of distributions).

The space of distributions $\mathcal{D}^{\prime}(\mathcal{U})$ is defined as the topological dual of $\mathcal{D}(\mathcal{U})$.

Moreover a distribution is a linear functional ${ }^{8} u: \mathcal{D}(\mathcal{U}) \rightarrow \mathbb{C}$, such that for each sequence of test functions $f_{n} \stackrel{\mathcal{D}}{\rightarrow} 0$, one has $u\left(f_{n}\right) \rightarrow 0$. In the following, the notions of support and singular support of a distribution are defined. These notions will be used in order to characterize the singular structure later.

\footnotetext{
${ }^{8}$ The action of the distribution $u$ on a test functions $f$ is also denoted $\langle u, f\rangle$.
} 
Definition 3.4.3 (Support).

The support of a distribution $u \in \mathcal{D}^{\prime}(\mathcal{U})$, denoted by supp $\mathrm{u}$, is the smallest closed set $K$ such that $\left.u\right|_{\mathcal{U} \backslash \mathcal{K}}=0$.

Definition 3.4.4 (Singular support).

The singular support of a distribution $u \in \mathcal{D}^{\prime}(\mathcal{U})$, denoted by $\operatorname{sing} \operatorname{supp} u$, is the smallest closed subset $K$ such that $\left.u\right|_{\mathcal{U} \backslash \mathcal{K}} \in C^{\infty}(\mathcal{U} \backslash \mathcal{K})$.

We have everything we need to correctly define the Dirac $\delta$ distribution which is the first example of distribution which is not a function.

Definition 3.4.5 (Dirac delta distribution $\delta$ ). The Dirac delta distribution $\delta_{x_{0}} \in \mathcal{D}^{\prime}(\mathcal{U})$ at a point $x_{0} \in \mathcal{U}$ is defined as

$$
\delta_{x_{0}}: \mathcal{D}(\mathcal{U}) \rightarrow \mathbb{C}, \quad f \mapsto f\left(x_{0}\right)
$$

The $\delta$ distribution is singular and has singular support given by $\left\{x_{0}\right\}$.

The most popular linear functional is the integral. It is therefore natural to define distributions from locally integrable functions. In effect let $g \in L_{\mathrm{loc}}^{1}(\mathcal{U})$. Then

$$
f \mapsto u_{g}(f)=\int_{\mathcal{U}} d x g(x) f(x)
$$

defines a distribution in $\mathcal{D}^{\prime}(\mathcal{U})$ and the map $g \rightarrow u_{g}$ is injective. Using duality, one can define operations on distributions. The most relevant are listed below.

Definition 3.4.6 (Product with a function).

The product of a distribution with a smooth function $g \in C^{\infty}$ is defined by

$$
(g \cdot u)(f)=u(g \cdot f)
$$

Definition 3.4.7 (Derivative).

The derivative of a distribution $u \in \mathcal{D}^{\prime}$ is defined by

$$
\left(\partial^{\alpha} u\right)(f)=(-1)^{|\alpha|} u\left(\partial^{\alpha} f\right)
$$

where $\alpha$ is a multiindex.

The consistency of the last definition can be verified for distributions constructed from smooth functions. In fact, for the simple case $\mathcal{U}=\mathbb{R}$ it is trivial to verify that the minus sign comes from the integration by parts

$$
\int_{-\infty}^{\infty} d x f^{\prime}(x) g(x)=[f(x) g(x)]_{-\infty}^{\infty}-\int_{-\infty}^{\infty} d x f(x) g^{\prime}(x)
$$


Definition 3.4.8 (Pullback).

Let $g$ be a smooth function from $\mathcal{U}$ to $\mathcal{V}$ and $f$ a test function in $\mathcal{D}(\mathcal{V})$. Then the pullback $g^{*} f \in C^{\infty}(\mathcal{U})$ is defined as

$$
g^{*} f(x)=f(g(x))
$$

Similarly, by duality, let $u \in \mathcal{D}^{\prime}(\mathcal{V})$ be a distribution. If $g$ is a submersion, then the pullback $g^{*} u \in \mathcal{D}^{\prime}(\mathcal{U})$ is well defined as

$$
g^{*} u(f)=u\left(f \circ g^{-1}\left|\operatorname{det}\left(g^{-1}\right)\right|\right)
$$

In order to introduce the concept of Fourier transform of a distribution, we define the space of tempered distributions in open subsets of $\mathbb{R}^{n}$. First, the Schwartz space is defined as the functional space of all functions whose derivatives are rapidly decreasing.

Definition 3.4.9 (Schwartz space). The Schwartz space is defined as

$$
S=\left\{f: \mathbb{R}^{n} \rightarrow \mathbb{C}, \sup _{x \in \mathbb{R}^{n}}\left|x^{\alpha} \partial^{\beta} f\right|<\infty, \forall \alpha, \beta \text { multiindex }\right\}
$$

Tempered distributions are defined as the space of continuous linear functional on $\mathcal{S}\left(\mathbb{R}^{n}\right)$.

Definition 3.4.10 (Schwartz distributions (tempered distributions)). The space of tempered distributions $\mathcal{S}^{\prime}\left(\mathbb{R}^{n}\right)$ is defined as the topological dual of $\mathcal{S}\left(\mathbb{R}^{n}\right)$.

To conclude, the Fourier transform of a distribution is defined.

Definition 3.4.11. Let $u \in \mathcal{S}^{\prime}$. Then it is possible to define the Fourier transform $\hat{u}$ as

$$
\hat{u}(f)=u(\hat{f}), \quad \forall f \in \mathcal{S} .
$$

where $\mathcal{S}$ is the Schwartz space.

\section{Scaling and renormalization of distributions}

In quantum field theory, in order to renormalize divergent quantities as loop integrals, it is important to identify their divergence and degree of singularity. In principle it is possible to have an analogous characterization for distributions. In this case the problem of renormalization translates into the problem of extension of singular distributions to the whole domain [110-112]. Let us introduce the notions of scaling degree and singular order. 
A possible definition for the scaling of a function $f: \mathbb{R}^{n} \rightarrow \mathbb{C}$ about 0 is $\operatorname{sd}(f):=$ $\inf _{\omega}\left\{\omega: \lim _{\lambda \rightarrow 0} \lambda^{\omega} f(\lambda x)=0,0<|x|<+\infty\right\}$. By duality we can imagine a similar definition for distributions.

Definition 3.4.12 (Scaling degree [113]).

The Scaling Degree sd of a distribution $u \in \mathcal{D}^{\prime}$ is defined by

$$
\operatorname{sd}(u):=\inf _{\omega}\left\{\omega: \lim _{\lambda \rightarrow 0} \lambda^{\omega} u_{\lambda}=0\right\}
$$

The singular properties of the distribution will also depend on the dimensionality of the space. Let us include this in our definition and define the singular order of a distribution.

Definition 3.4.13 (Singular order).

The Singular Order or Divergence Degree div of a distribution $u \in \mathcal{D}^{\prime}$ is defined by

$$
\operatorname{div}(u):=\operatorname{sd}(u)-n
$$

These definitions can be applied to the Dirac delta distribution $\delta$ in $\mathbb{R}^{n}$. It is a trivial exercise to check that $\operatorname{sd}(\delta)=n$ and $\operatorname{div}(\delta)=0$. Similarly on $\mathbb{R}^{4}$ the scaling of $\Delta_{F}=\frac{1}{x^{2}}$ about $x=0$ is $\operatorname{sd}\left(\Delta_{F}\right)=2$ and $\operatorname{div}\left(\Delta_{F}\right)=-2$. The natural question here is about the possibility of extension of a distribution where it is not well-defined.

Let's suppose that $u$ is not defined at the singular point $x=0$, i.e. $u \in \mathcal{D}^{\prime}\left(\mathbb{R}^{n} \backslash\{0\}\right)$. An extension of $u$ to the whole domain should satisfy these two properties: it should have the same singular structure as $u$ and the same behavior for $x \neq 0$. Therefore we define the extension of a distribution as

Definition 3.4.14 (Extension).

Let $u \in \mathcal{D}^{\prime}\left(\mathbb{R}^{n} \backslash\{0\}\right)$ be a distrubution. An extension $\tilde{u}$ of $u$ in 0 satisfies

- $\tilde{u}(f)=u(f), \forall f \in \mathcal{D}\left(\mathbb{R}^{n} \backslash\{0\}\right)$

- $\operatorname{sd}(\tilde{u})=\operatorname{sd}(u)$

The possibility to extend a distribution is given by the following theorem

Theorem 3.4.1 (Extension or renormalization of a distribution [107]). Let $u \in \mathcal{D}^{\prime}\left(\mathbb{R}^{n} \backslash\{0\}\right)$ be a distrubution. Then

- If $\operatorname{div}(u)<0$ there exists a unique extension $\tilde{u}$ such that $\operatorname{sd}(\tilde{u})=\operatorname{sd}(u)$

- If $0 \leq \operatorname{div}(u)<\infty$ there exist extensions $\tilde{u}$ of $u$ with $\operatorname{sd}(\tilde{u})=\operatorname{sd}(u)$ and they differ by a term of the form $\sum_{|\alpha| \leq \operatorname{div}(u)} c_{\alpha} \delta^{(\alpha)}$ 
In the case where the singular order is smaller than 0, let's discuss how to construct the extension from the singular distribution $[25,26,114]$. Let $u \in \mathcal{D}\left(\mathbb{R}^{n} \backslash\{0\}\right)$ and $\operatorname{div}(u)<0$, the extension of $u$ to $\mathcal{D}\left(\mathbb{R}^{n}\right)$ is constructed through a sequence of smooth functions $\theta_{n}$ such that $\theta_{n}(0)=0$ and $\theta_{n}(x)=1$ for $x \in U_{n}^{c}$, where $U_{n}$ are neighborhoods of the origin and $U_{n}^{c}$ denotes the complement of $U_{n}$. In the limit $n \rightarrow \infty$, the sequence $\theta_{n}$ is required to converge to the following limit

$$
\theta_{n} \stackrel{n \rightarrow \infty}{\longrightarrow} \theta_{\infty}= \begin{cases}0 & , \quad x=0 \\ 1 & , \quad \text { otherwise }\end{cases}
$$

The extended distribution is given by $\tilde{u}=\lim _{n \rightarrow \infty} \theta_{n} u$ and it is independent of the choice of the sequence $\left\{\theta_{n}\right\}_{n \in \mathbb{N}}$.

An example of $\theta_{n}$ functions is $\theta_{n}(x)=1-e^{\frac{\left(2^{n} x\right)^{2}}{\left(2^{n} x\right)^{2}-1}} \chi_{\left[-2^{-n}, 2^{-n}\right]}$ and it shown in Figure 3.1.

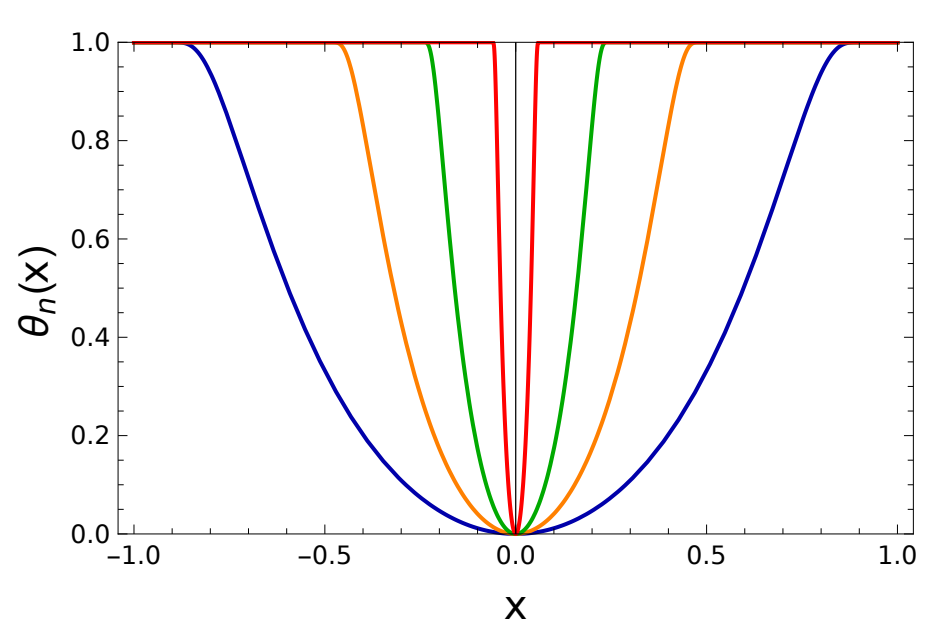

Figure 3.1: Cutoff function $\theta_{n}(x)=1-e^{\frac{\left(2^{n} x\right)^{2}}{\left(2^{n} x\right)^{2}-1}} \chi_{\left[-2^{-n}, 2^{-n}\right]}$ for different parameters: $n=0.1$ (blue line), $n=1$ (orange line), $n=2$ (green line) and $n=4$ (red line).

The extension theorem guarantees a unique extension on test functions that vanish at the origin up to order $\omega$.

Definition 3.4.15 ( $W$-operation).

Let $\mathcal{D}^{\omega}\left(\mathbb{R}^{n}\right) \subset \mathcal{D}\left(\mathbb{R}^{n}\right)$ be the subspace of function vanishing up to order $\omega$ at 0 . The function $W$ is a projection into that subspace

$$
W_{\omega}: \mathcal{D}\left(\mathbb{R}^{n}\right) \rightarrow \mathcal{D}^{\omega}\left(\mathbb{R}^{n}\right), \quad \varphi \mapsto W_{\omega} \varphi
$$


where

$$
\left(W_{\omega} \varphi\right)(x)=\varphi(x)-w(x) \sum_{|\alpha| \leq \omega} \frac{x^{\alpha}}{\alpha !}\left(\partial^{\alpha} \frac{\varphi}{w}\right)(0),
$$

with $w \in \mathcal{D}\left(\mathbb{R}^{n}\right), w(0) \neq 0$.

Now we are able to recall the main theorem of this section. It will be used as the reference for the construction of extensions of distributions and to identify the extension freedom that one has.

Theorem 3.4.2 (Main Theorem, renormalization freedom [115]).

Let $u_{0} \in \mathcal{D}^{\prime}\left(\mathbb{R}^{n} \backslash\{0\}\right)$ be a distribution with singular order $\omega$. Given a $W_{\omega}$ operation and constants $C^{\alpha} \in \mathbb{C}$, then there is one distribution $u \in \mathcal{D}^{\prime}\left(\mathbb{R}^{n}\right)$ with singular order $\omega$ such that

- $u(\varphi)=u_{0}(\varphi), \forall \varphi \in \mathcal{D}\left(\mathbb{R}^{n} \backslash\{0\}\right)$

- $u\left(w x^{\alpha}\right)=C^{\alpha}, \alpha \leq \omega$.

The extension $u$ is then given by

$$
u(\varphi)=u_{0}^{\mathrm{ext}}\left(W_{\omega} \varphi\right)+\sum_{|\alpha| \leq \omega} \frac{C^{\alpha}}{\alpha !}\left(\partial^{\alpha} \frac{\varphi}{w}\right)(0) .
$$

We point out that $w$ is a function used in order to make quantities like $u\left(w x^{\alpha}\right)$ meaningful. In the following we will call renormalization the extension of a distribution and renormalization freedom, the freedom in the choice of the constants $C^{\alpha}$.

The advantage of this construction based on distribution theory is that it is deeply local in spirit, as it does not depend on the global structure of $\mathbb{R}^{n}$. It can thus be easily generalized to the case of a quantum field theory in curved spacetime $[112,116]$.

\section{Singular structure and Wavefront Set}

This introduction about renormalization of distribution is concluded with the characterization of the singular structure of distributions [117] and of propagators [108]. It was first observed by Radzikowski [118] that microlocal analysis and the definition of the wavefront are well suited in order to formally describe the singularities of distributions on curved spacetimes [119, 120].

In quantum field theory, divergences arise when we consider loop diagrams. One possibility is the tadpole, $\Delta(0)$, which is not considered in the algebraic QFT since it vanishes after a proper choice of normal ordering. Another possibility that will be considered in the last part of this section is given by the product of two or more 
propagators at the same spacetime point, e.g. $\Delta^{2}(x-y)$. In the formal language, this product is ill-defined because we are trying to multiply two distributions that are too singular. We will discuss this issue here.

The Wavefront Set (WF) is the quantity that contains the information about the singular properties of the distribution, i.e. the singularities and all the directions in Fourier space that contribute to the singular support. It is defined as

Definition 3.4.16 (Wavefront Set). For a distribution $u \in \mathcal{D}^{\prime}(\mathcal{U})$ the wavefront set $\mathrm{WF}(u)$ is the complement in $\mathcal{U} \times \mathbb{R}^{n} \backslash\{0\}$ of the set of points $(x, \xi) \in \mathcal{U} \times \mathbb{R}^{n} \backslash\{0\}$ such that there exist

- a function $f \in \mathcal{D}(\mathcal{U})$ with $f(x)=1$

- an open conic neighborhood $\Gamma$ of $\xi$ with

$$
\sup _{\xi \in \Gamma}(1+|\xi|)^{N}|\widehat{f \cdot u(\xi)}|<\infty, \forall N \in \mathbb{N}_{0}
$$

The WF is a local concept and is related to the possibility of defining the product of two singular distributions. The usual multiplication of functions $f_{1}, f_{2} \in \mathcal{C}(\mathcal{U})$ can be understood as the tensor product $f_{1} \otimes f_{2}$ restricted to the diagonal in $\mathcal{U} \times \mathcal{U}$, i.e. $\left(f_{1} \otimes f_{2}\right)(x, x)=f_{1}(x) f_{2}(x)$. This remains valid also for distributions, with the difference that the restriction to the diagonal is not always well-defined. The Hörmander criterion gives a sufficient condition in order to have a consistent definition [108] of the product of two distributions.

Corollary 3.4.2.1 (Hörmander Criterion). The product of two distributions $u_{1}, u_{2} \in$ $\mathcal{D}^{\prime}(\mathcal{U})$ can be defined as the restriction of $u_{2} \otimes u_{1}{ }^{9}$ to the diagonal if the following condition is satisfied

$$
(x, \xi) \in \mathrm{WF}\left(u_{1}\right) \Rightarrow(x,-\xi) \notin \mathrm{WF}\left(u_{2}\right) .
$$

The corollary says that one can define a meaningful product of two distributions because the growth of $u_{1}$ in the $\xi$ direction is suppressed by the decay of $u_{2}$ in the $-\xi$ direction. When the Hörmander criterion is not satisfied it might be possible to define the product of two distributions as the renormalized product.

As a trivial example we can imagine the product of two $\Delta_{F}=\frac{1}{x^{2}}$ distributions on a 4 dimensional space. The product $\Delta_{F}^{2} \in \mathcal{D}\left(\mathbb{R}^{4} \backslash\{0\}\right)$ is singular at 0 and has $\operatorname{sd}\left(\Delta_{F}^{2}\right)=4$ and therefore $\operatorname{div}\left(\Delta_{F}^{2}\right)=0$. This means that the extension of $\Delta_{F}^{2}$ to $\mathcal{D}\left(\mathbb{R}^{4}\right)$ exists but is not unique. Let $\tilde{\Delta}_{F}^{2}$ and $\bar{\Delta}_{F}^{2}$ be two extensions, then there exists

\footnotetext{
${ }^{9}$ Here the term restriction means the pullback of $u_{2} \otimes u_{1}$ under the diagonal map.
} 
a constant $c$ such that $\tilde{\Delta}_{F}^{2}=\bar{\Delta}_{F}^{2}+c \delta$. This is an example of renormalization of the propagator $\Delta_{F}$ in the language of distributions where $c$ represents the freedom in the renormalization scheme. In Figure 3.2 the scheme of the singular structure of the most important propagators used in physics is shown.

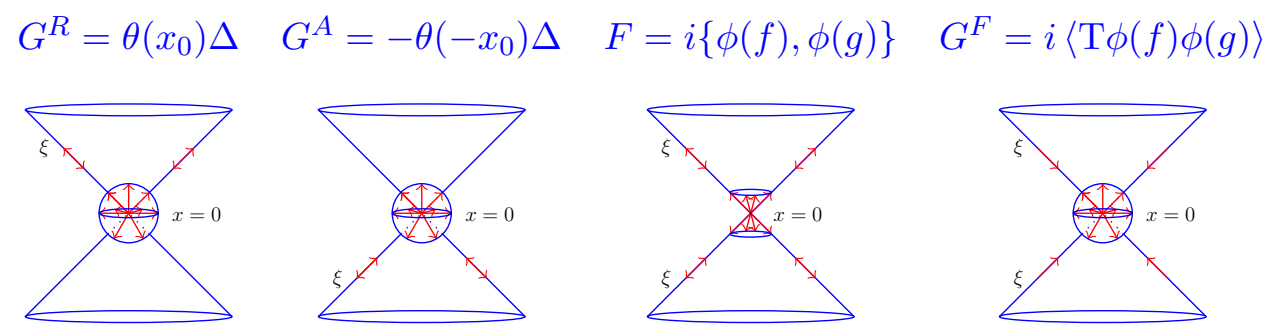

Figure 3.2: Singular structure of the advanced and retarded propagators $G^{A / R}$, of the Schwinger propagator $F$ and of the Feynman propagator $G^{F}$ in Minkowski spacetime. The commutator is denoted by $\Delta=i[\phi(f), \phi(g)]$.

This concludes our discussion about the Epstein-Glaser renormalization in quantum field theory. As stated before, this construction can be easily generalized for theories defined in curved spacetime since it is based only on local concepts. Moreover the construction of the renormalized distribution in eq. (3.52) is very general and it is not based on any physically motivated scheme. The high level of abstraction reflects on the difficulties in explicitly renormalizing concrete examples of radiative contributions for general spacetimes. In this regard, progress has been done in [38] where an explicit regularization scheme in position space was invented, with the peculiarity that it is compatible with the algebraic quantum field theory approach. 


\section{CHAPTER 4}

\section{| THE SCHWINGER AND KELDYSH FORMALISM}

Primordial fluctuations are an important part of the early universe scenario and they provide the initial space inhomogeneities of the universe required in order to explain the observed temperature anisotropies in the cosmic microwave background and the large scale structure of the universe. Differently from the standard approach in particle physics, the theory describing the primordial universe contains an explicit time dependence arising from the expanding universe. In this picture the cosmological observables acquire an explicit time dependence inherited from the background. In this perspective we are interested in a formalism that allows to follow the time-evolution of expectation values from an initial state including the quantum effects of the theory. Moreover for certain systems that are not invariant under the Poincaré symmetries, the scattering matrix [121-124] cannot be defined consistently [125]. This approach is different to the traditional one where the focus is on the understanding of scattering properties of quantum particles.

The Schwinger and Keldysh formalism [126-131] is the appropriate formalism in order to study the time-evolution of cosmological observables including the radiative corrections even for systems where the time-translational symmetry is broken, as it happens for example in an expanding universe. These methods are well-known in the condensed matter community and often applied to non-equilibrium quantum theories [132].

The Schwinger and Keldysh formalism ${ }^{1}$ is introduced in Section 4.1 for timedependent setups [133] with cosmological backgrounds. In particular the theoretical tools used in order to obtain a perturbative expression for the expectation values are

\footnotetext{
${ }^{1}$ The Schwinger and Keldysh formalism is also called the closed-time-path (CTP) formalism or the in-in formalism.
} 
discussed. In Section 4.2 the system is evaluated perturbatively and the closed-timepath propagators are discussed. The explicit form of the propagators for a Minkowski and de-Sitter background for massless and massive theories are given in Section 4.3.

\subsection{Introduction}

In a Lorentz-invariant quantum field theory one can break explicitly the Poincaré symmetry by considering for example systems that are governed by a time-dependent Hamiltonian $H(t)$ or a time-dependent background $g^{\mu \nu}$ for the case of an expanding Friedmann Lemaître Robertson Walker universe. Here the metric is considered as a classical background. The time dependence on the scale factor $a(t)$ then breaks the Poincaré symmetry and we cannot rely on in-out approach of quantum field theory.

In the closed-time-path formalism one considers a system described by a timedependent Hamiltonian $H(t)$ in a state defined by the density matrix $\rho(t)$. The expectation value of an observable $\mathcal{O}$ at time $t>t_{\text {in }}$ is given by

$$
\langle\mathcal{O}(t)\rangle=\operatorname{Tr}(\rho(t) \mathcal{O}(t))
$$

For practical reasons, it is convenient to work on the interaction picture where the time-evolution of the density matrix is described by the Liouville equation

$$
\left\{\begin{array}{l}
i \frac{\partial \rho(t)}{\partial t}=\left[\hat{H}_{I}(t), \rho(t)\right], \\
\rho\left(t_{\text {in }}\right)=\rho_{\text {in }}
\end{array}\right.
$$

where the full Hamiltonian is split into the free and interacting part $\hat{H}(t)=\hat{H}_{0}(t)+$ $\hat{H}_{I}(t)$. The solution is given in terms of the time-evolution operator $U_{I}\left(t, t_{\text {in }}\right)$ introduced as the solution of the Dyson equation

$$
\left\{\begin{array}{l}
i \frac{\partial U_{I}\left(t, t_{\mathrm{in}}\right)}{\partial t}=\hat{H}_{I}(t) U_{I}\left(t, t_{\mathrm{in}}\right) \\
U_{I}\left(t_{\mathrm{in}}, t_{\mathrm{in}}\right)=1
\end{array} .\right.
$$

Therefore, $\rho(t)$ can be expressed in terms of $U, U^{\dagger}$ and the initial condition $\rho_{\text {in }}$

$$
\rho(t)=U_{I}\left(t, t_{\text {in }}\right) \rho_{\text {in }} U_{I}^{\dagger}\left(t, t_{\text {in }}\right)
$$

The last equation can be verified by showing that (4.4) is a solution of (4.2). To compute $\rho$ at time $t$ it is sufficient to find the solution of equation (4.3) which is 
formally given by

$$
U_{I}\left(t, t_{\mathrm{in}}\right)=\mathrm{T} e^{-i \int_{t_{\mathrm{in}}}^{t} d \tau \hat{H}_{I}(\tau)}
$$

where T means the time-ordered product. The expression for $\rho$ follows immediately

$$
\rho(t)=\left(\mathrm{T} e^{-i \int_{t_{\mathrm{in}}}^{t} d \tau \hat{H}_{I}(\tau)}\right) \rho_{\mathrm{in}}\left(\mathrm{T} e^{-i \int_{t_{\mathrm{in}}}^{t} d \tau \hat{H}_{I}(\tau)}\right)^{\dagger}
$$

Now we have an explicit expression for $\langle\mathcal{O}(t)\rangle$

$$
\langle\mathcal{O}(t)\rangle=\operatorname{Tr}\left\{\rho_{\mathrm{in}}\left(\mathrm{T} e^{-i \int_{t_{\mathrm{in}}}^{t} d \tau \hat{H}_{I}(\tau)}\right)^{\dagger} \mathcal{O}(t)\left(\mathrm{T} e^{-i \int_{t_{\mathrm{in}}}^{t} d \tau \hat{H}_{I}(\tau)}\right)\right\}
$$

that can be understood as the time-evolution from the initial time $t_{\text {in }}$ up to time $t$, where the observable $\mathcal{O}$ is evaluated. Then the system evolves backwards to the initial time.

It is convenient to extend the time-evolution to $t=+\infty$. A common trick is to insert $\mathbb{I}=U_{I}^{\dagger}(\infty, t) U_{I}(\infty, t)$ to the left of $\mathcal{O}(t)$. Then the expectation-value reads

$$
\langle\mathcal{O}(t)\rangle=\operatorname{Tr}\left\{\rho_{\mathrm{in}}\left(\mathrm{T} e^{-i \int_{t_{\mathrm{in}}}^{\infty} d \tau \hat{H}_{I}(\tau)}\right)^{\dagger}\left(\mathrm{T} e^{-i \int_{t}^{\infty} d \tau \hat{H}_{I}(\tau)}\right) \mathcal{O}(t)\left(\mathrm{T} e^{-i \int_{t_{\mathrm{in}}}^{t} d \tau \hat{H}_{I}(\tau)}\right)\right\}
$$

that represents the time-evolution along the closed time contour $\mathcal{C}$ shown in Figure 4.1. It should be noted that the observable $\mathcal{O}(t)$ is evaluated in the forward part of the contour $\mathcal{C}$, because we have inserted the identity operator $U_{I}^{\dagger}(+\infty, t) U_{I}(+\infty, t)$ to the left of $\mathcal{O}(t)$. The same manipulations can be performed by inserting the identity operator to the right of $\mathcal{O}(t)$. In this case, the same result is obtained with the only exception that $\mathcal{O}(t)$ is evaluated in the backward part of the contour. Conventionally, all the observables are evaluated in the forward part.

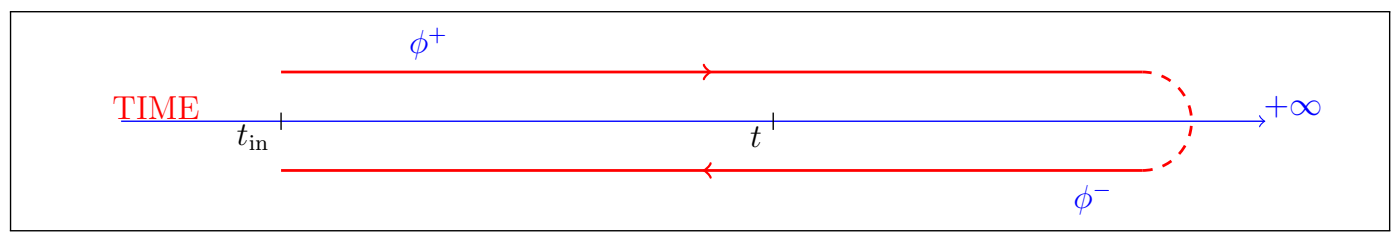

Figure 4.1: Closed-time contour $\mathcal{C}$ representing the time-ordering $T_{\mathcal{C}}$.

From this expression, perturbation theory can be applied by defining Feynman rules for the computation of perturbative corrections. Indeed one can define the time-ordering $T_{\mathcal{C}}$ along the contour given in Figure 4.1 and split the field $\phi$ in two components $\phi^{ \pm}$, where the + component propagates along the upper part of the contour and is governed by the Hamiltonian $\hat{H}_{I}^{+}(t)=\hat{H}_{I}\left[\phi^{+}\right]$and the - component propagates in the lower part 
of the contour and is governed by $\hat{H}_{I}^{-}(t)=\hat{H}_{I}\left[\phi^{-}\right]$. Finally the expectation value (4.8) can be expressed as

$$
\langle\mathcal{O}(t)\rangle=\operatorname{Tr}\left\{\rho_{\mathrm{in}} \mathrm{T}_{\mathcal{C}}\left[\mathcal{O}^{+}(t) e^{-i \int_{t_{\mathrm{in}}}^{+\infty} d \tau\left[\hat{H}_{I}^{+}(\tau)-\hat{H}_{I}^{-}(\tau)\right.}\right]\right\}
$$

where the operator $\mathcal{O}^{+}$is evaluated in the upper part of the contour.

\subsection{Perturbation theory}

By supposing that the interaction in eq. (4.9) is small with respect to the free part, one can treat the expectation value perturbatively.

Since the two components propagate on the two independent parts of the contour, between $t_{\text {in }}$ to infinite time or opposite, we can exploit the traditional Feynman rules introduced in the in-out approach of quantum field theory [84] in order to compute the perturbative correction to the expectation value. The Feynman rules for a simple scalar field model with quartic self-interaction and for a hybrid model with two scalar fields with quartic symmetric interaction are given in the Appendix A.

In this framework, for every scalar field of the theory there are four possible contractions of the field components and therefore four propagators

$$
G^{ \pm \pm}(x, y)=i\left\langle\phi^{ \pm}(x) \phi^{ \pm}(y)\right\rangle
$$

The Green functions can be expressed more explicitly as

$$
\begin{aligned}
& G^{+-}(x, y)=i\langle\phi(y) \phi(x)\rangle, \\
& G^{-+}(x, y)=i\langle\phi(x) \phi(y)\rangle, \\
& G^{++}(x, y)=\theta\left(x^{0}-y^{0}\right) G^{-+}(x, y)+\theta\left(y^{0}-x^{0}\right) G^{+-}(x, y), \\
& G^{--}(x, y)=\theta\left(x^{0}-y^{0}\right) G^{+-}(x, y)+\theta\left(y^{0}-x^{0}\right) G^{-+}(x, y) .
\end{aligned}
$$

The 4 propagators are not independent because the two field's components satisfy the boundary condition $\phi^{+}(\infty)=\phi^{-}(\infty)$. Indeed, they are connected through the simple relation

$$
G^{++}(x, y)+G^{--}(x, y)=G^{+-}(x, y)+G^{-+}(x, y)
$$

and can be regrouped together in a matrix form

$$
\mathbf{G}(x, y)=\left(\begin{array}{ll}
G^{++}(x, y) & G^{+-}(x, y) \\
G^{-+}(x, y) & G^{--}(x, y)
\end{array}\right) .
$$


Since the two components of the field $\phi$ are not two independent degrees of freedom, one can transform $\phi^{+}$and $\phi^{-}$into a more convenient basis. We define $\mathbf{R}$ as the matrix

$$
\mathbf{R}=\left(\begin{array}{cc}
1 / 2 & 1 / 2 \\
1 & -1
\end{array}\right)
$$

representing the change of basis. The new fields $\phi^{(1)}$ and $\phi^{(2)}$ and the new propagators $\mathbf{G}_{\mathbf{R}}$ are

$$
\left(\begin{array}{l}
\phi^{(1)} \\
\phi^{(2)}
\end{array}\right)=\mathbf{R}\left(\begin{array}{c}
\phi^{+} \\
\phi^{-}
\end{array}\right)=\left(\begin{array}{c}
\left(\phi^{+}+\phi^{-}\right) / 2 \\
\phi^{+}-\phi^{-}
\end{array}\right)
$$

and

$$
\mathbf{G}_{\mathbf{R}}=\mathbf{R} \mathbf{G} \mathbf{R}^{T}=:\left(\begin{array}{cc}
i F & G^{R} \\
G^{A} & 0
\end{array}\right)
$$

The new basis is called the Schwinger basis and has the peculiarity that the $\phi^{(2)}-\phi^{(2)}$ contraction is always vanishing. We recognize $G^{R}$ and $G^{A}$ as the retarded and advanced propagators and $F$ as the Schwinger or Hadamard propagator ${ }^{2}$. In the Schwinger basis the propagators are given by

$$
\begin{aligned}
F(x, y) & =-\frac{i}{2}\left(G^{-+}(x, y)+G^{+-}(x, y)\right) \\
G^{R}(x, y) & =\theta\left(x_{0}-y_{0}\right)\left(G^{-+}(x, y)-G^{+-}(x, y)\right) \\
G^{A}(x, y) & =G^{R}(y, x) .
\end{aligned}
$$

Finally the three propagators are connected to the familiar Feynman propagator $G^{F}$

$$
G^{F}(x, y)=i\langle\mathrm{~T}[\phi(x) \phi(y)]\rangle=\frac{1}{2}\left(G^{R}(x, y)+G^{A}(x, y)\right)+i F(x, y) .
$$

In our analysis the radiative corrections to the expectations values are evaluated in the case of a spatially flat FRW metric. The spatial coordinates of the propagators can be Fourier trasformed in momentum space giving a dependence on 3 independent variables: the momentum $k$ and two times $t_{1}$ and $t_{2}$, i.e. they assume the form $G^{A / R}\left(k, t_{1}, t_{2}\right)$ or $F\left(k, t_{1}, t_{2}\right)$. The invariance under spatial translations insures that

$$
G^{A / R}\left(\vec{x}_{1}, t_{1}, \vec{x}_{2}, t_{2}\right)=G^{A / R}\left(\vec{x}_{1}-\vec{x}_{2}, t_{1}, t_{2}\right)
$$

\footnotetext{
${ }^{2}$ In [134] the Schwinger function $-i G^{(1)}$ is defined as $-2 i F$.
} 


\subsection{Closed-time-path propagators}

In the following, the closed-time-path propagators are examined for different backgrounds and in different limits. First, the propagators of a scalar field theory in Minkowski spacetime are shown for an initial state given by the vacuum state of the free theory. Then, the propagators in de-Sitter spacetime are presented with the initial state given by the Bunch-Davies vacuum [135]. This section ends with the discussion about the WKB propagators that are a good approximation for very massive theories.

\section{Minkowski propagators}

The propagators (4.10) are presented for a scalar field theory in Minkowski spacetime where the density metric $\rho_{\text {in }}$ is given by the free vacuum state. The +- contraction is given by [124]

$$
\begin{aligned}
G^{+-}(x, y) & =i\langle\phi(y) \phi(x)\rangle=i \int \frac{d^{3} k}{(2 \pi)^{3}} \frac{1}{2 w_{k}} e^{-i k \cdot(y-x)} \\
& =i \int \frac{d^{3} k}{(2 \pi)^{3}} e^{-i k \cdot(x-y)}\left[\frac{1}{2 w_{k}} e^{i w_{k} \cdot\left(x^{0}-y^{0}\right)}\right]
\end{aligned}
$$

where $w_{k}=\sqrt{k^{2}+m^{2}}$. The same calculation can be performed for $G^{-+}$. From this expression we recognize the 3 -dimensional Fourier modes

$$
\begin{aligned}
& G^{+-}\left(w_{k}, x_{0}, y_{0}\right)=\frac{i}{2 w_{k}} e^{+i w_{k} \cdot\left(x^{0}-y^{0}\right)}, \\
& G^{-+}\left(w_{k}, x_{0}, y_{0}\right)=\frac{i}{2 w_{k}} e^{-i w_{k} \cdot\left(x^{0}-y^{0}\right)} .
\end{aligned}
$$

Finally the expressions for the last two propagators read

$$
\begin{aligned}
& G^{++}\left(w_{k}, x_{0}, y_{0}\right)=\frac{i}{2 w_{k}}\left\{e^{+i w_{k} \cdot\left(x^{0}-y^{0}\right)}-2 i \theta\left(x^{0}-y^{0}\right) \sin \left[w_{k}\left(x^{0}-y^{0}\right)\right]\right\} \\
& G^{--}\left(w_{k}, x_{0}, y_{0}\right)=\frac{i}{2 w_{k}}\left\{e^{-i w_{k} \cdot\left(x^{0}-y^{0}\right)}+2 i \theta\left(x^{0}-y^{0}\right) \sin \left[w_{k}\left(x^{0}-y^{0}\right)\right]\right\}
\end{aligned}
$$

The previous propagators can be easily transformed into the Schwinger basis (4.19). The explicit expressions are

$$
\begin{aligned}
F\left(w_{k}, t_{1}, t_{2}\right) & =\frac{\cos \left(w_{k}\left(t_{1}-t_{2}\right)\right)}{2 w_{k}} \\
G^{R}\left(w_{k}, t_{1}, t_{2}\right) & =\theta\left(t_{1}-t_{2}\right) \frac{\sin \left(w_{k}\left(t_{1}-t_{2}\right)\right)}{w_{k}} \\
G^{A}\left(w_{k}, t_{1}, t_{2}\right) & =G^{R}\left(w_{k}, t_{2}, t_{1}\right) .
\end{aligned}
$$




\section{De-Sitter propagators}

In de-Sitter spacetime, for small masses, the propagators in momentum space can be expressed in terms of the Bessel functions $J_{\nu}$ [136-138]

$$
\begin{aligned}
G^{-+}\left(k, \tau_{1}, \tau_{2}\right) & =\frac{H^{2} \pi J_{\nu}(z) J_{-\nu}\left(z^{\prime}\right)}{2 \sin (\pi \nu)}\left(\tau_{1} \tau_{2}\right)^{3 / 2} \\
G^{+-}\left(k, \tau_{1}, \tau_{2}\right) & =\frac{H^{2} \pi J_{-\nu}(z) J_{\nu}\left(z^{\prime}\right)}{2 \sin (\pi \nu)}\left(\tau_{1} \tau_{2}\right)^{3 / 2}
\end{aligned}
$$

where $\tau_{1,2}$ is the conformal time, $z=-k \tau_{1}, z^{\prime}=-k \tau_{2}$, and

$$
\nu=\sqrt{\frac{9}{4}-\frac{m^{2}}{H^{2}}}, \quad \text { where } m \text { is the field's mass. }
$$

In the case of a massless field $\nu=3 / 2$ the propagators assume a simple form in terms of the Hankel functions $H_{\frac{3}{2}}^{(1)}, H_{\frac{3}{2}}^{(2)}$ as

$$
\begin{aligned}
& G^{-+}\left(k, \tau_{1}, \tau_{2}\right)=\frac{i \pi H^{2}}{4} H_{3 / 2}^{(1)}(z) H_{3 / 2}^{(2)}\left(z^{\prime}\right)\left(\tau_{1} \tau_{2}\right)^{3 / 2}, \\
& G^{+-}\left(k, \tau_{1}, \tau_{2}\right)=\frac{i \pi H^{2}}{4} H_{3 / 2}^{(2)}(z) H_{3 / 2}^{(1)}\left(z^{\prime}\right)\left(\tau_{1} \tau_{2}\right)^{3 / 2},
\end{aligned}
$$

where

$$
\begin{aligned}
H_{\frac{3}{2}}^{(1)}(z) & =\sqrt{\frac{2}{\pi z}} e^{i z}\left(\frac{1}{i z}-1\right), \\
H_{\frac{3}{2}}^{(2)}(z) & =\bar{H}_{\frac{3}{2}}^{(1)}(z) .
\end{aligned}
$$

For a massless scalar field theory in de-Sitter spacetime the propagators (4.31) and (4.32) in the Schwinger basis read

$$
\begin{aligned}
F\left(k, \tau_{1}, \tau_{2}\right) & =\frac{H^{2}}{2 k^{3}}\left(\left(1+k^{2} \tau_{1} \tau_{2}\right) \cos k\left(\tau_{1}-\tau_{2}\right)+k\left(\tau_{1}-\tau_{2}\right) \sin k\left(\tau_{1}-\tau_{2}\right)\right), \\
G^{R}\left(k, \tau_{1}, \tau_{2}\right) & =\frac{H^{2}}{k^{3}} \theta\left(\tau_{1}-\tau_{2}\right)\left(\left(1+k^{2} \tau_{1} \tau_{2}\right) \sin k\left(\tau_{1}-\tau_{2}\right)-k\left(\tau_{1}-\tau_{2}\right) \cos k\left(\tau_{1}-\tau_{2}\right)\right) .
\end{aligned}
$$

The expansion for small $|k \tau|$ is given by

$$
\begin{aligned}
F\left(k, \tau_{1}, \tau_{2}\right) & =\frac{H^{2}}{2 k^{3}}\left[1+\mathcal{O}\left(k^{2} \tau_{i}^{2}\right)\right], \\
G^{R}\left(k, \tau_{1}, \tau_{2}\right) & =\theta\left(\tau_{1}-\tau_{2}\right) \frac{H^{2}}{3 k^{3}}\left[k^{3}\left(\tau_{1}^{3}-\tau_{2}^{3}\right)+\mathcal{O}\left(k^{5} \tau_{i}^{5}\right)\right] .
\end{aligned}
$$


Finally, in the equal-time limit, the Schwinger propagator simplifies to

$$
F(k, \tau, \tau)=\frac{H^{2}}{2 k^{3}}\left(1+k^{2} \tau^{2}\right) .
$$

By integrating the two-point function in momentum space one realizes that the integral has to be regulated both in the infrared and in the ultraviolet regime. In order to cure the IR-behavior we consider

$$
\nu=\sqrt{\frac{9}{4}-\frac{m^{2}}{H^{2}}}
$$

which gives a deviation from the massless case $\nu=\frac{3}{2}$ given by a term proportional to $m \ll H$. By expanding the Hankel functions for a small mass and small momenta we get

$$
F(k, \tau, \tau)=\frac{H^{2}}{2 k^{3}}(k|\tau|)^{2 \varepsilon},
$$

with $\varepsilon=m^{2} / 3 H^{2}$.

Another interesting form for the propagator in de-Sitter spacetime is given in terms of the hypergeometric function in position space which is the exact two-point function for a massive field in de-Sitter spacetime [20]

$$
\begin{gathered}
G^{-+}\left(\tau_{1}, \tau_{2}, x_{1}, x_{2}\right)=\frac{H^{2} \Gamma(3 / 2-\nu) \Gamma(3 / 2+\nu)}{4 \pi^{2}}{ }_{2} F_{1}(3 / 2-\nu, 3 / 2+\nu, 2,1-r / 4) \\
\nu=\sqrt{\frac{9}{4}-\frac{m^{2}}{H^{2}}}, \quad r=\frac{\left(-\left(\tau_{1}-\tau_{2}\right)^{2}+\left|\mathbf{x}_{1}-\mathbf{x}_{2}\right|^{2}\right)}{\tau_{1} \tau_{2}}
\end{gathered}
$$

where the Hypergeometric function ${ }_{2} F_{1}$ is defined for $|z|<1$ by the power series

$$
{ }_{2} F_{1}(a, b, c, z)=\sum_{0}^{\infty} \frac{(a)_{n}(b)_{n}}{(c)_{n}} \frac{z^{n}}{n !} .
$$

In our analysis we preferred to work in the Schwinger basis because it highlights the physical properties of the field. The Hadamard propagator $F$ is directly connected to the real part of the contraction $G^{-+}$

$$
F\left(\tau_{1}, \tau_{2}, x_{1}, x_{2}\right)=\mathcal{R}\left[\frac{H^{2} \Gamma(3 / 2-\nu) \Gamma(3 / 2+\nu)}{4 \pi^{2}}{ }_{2} F_{1}(3 / 2-\nu, 3 / 2+\nu, 2,1-r / 4)\right] .
$$

Because there is no analytic formulation of the Fourier transform of this propagator, we proceed numerically and evaluate the massive propagator in Fourier space with numerical methods with Mathematica [139]. The Fourier transform is given by 


$$
F\left(k, \tau_{1}, \tau_{2}\right)=2 \pi \int_{0}^{\infty} d x x^{2} \frac{2 \sin (k x)}{k x} F\left(\tau_{1}, \tau_{2}, x, 0\right) .
$$

Unfortunately, the numerical integration is not always stable, especially for small masses, because of the appearance of oscillations that are enormously amplifying the numerical error. Nevertheless we found that the Fourier transformation is well-behaved for masses $m>H$. In the hybrid model where we will perform our calculations using the hypergeometric propagator we are considering very massive quantum fields and our procedure can be trusted.

In Figure 4.2 we show the behavour of the Schwinger propagator reconstructed from eq. (4.43) for different masses and we compared it with the massless propagator. In the plot we can observe that the hypergeometric function is well-behaved in the infrared regime as expected because it describes a massive theory. On the other hand the massless propagator diverges for $k=0$.

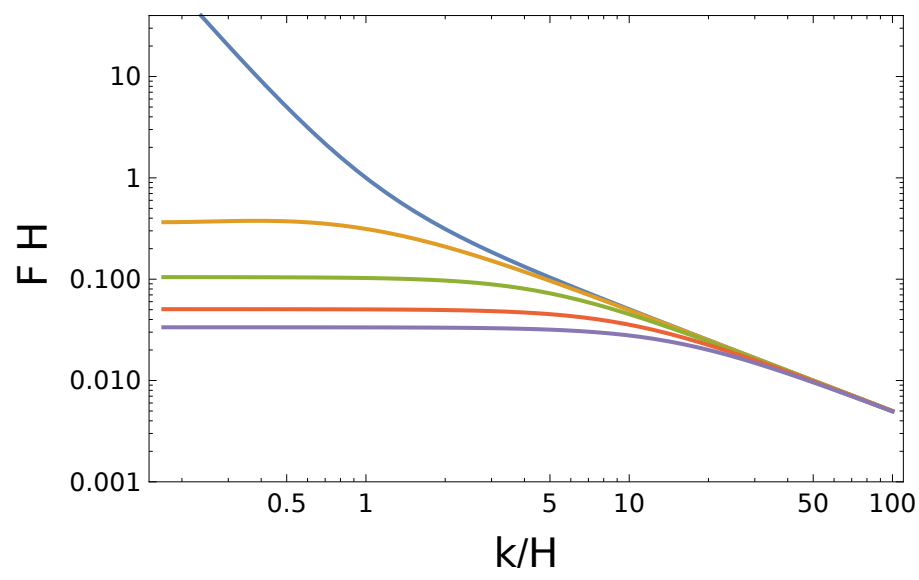

Figure 4.2: Hadamard propagators derived from the hypergeometric (full massive) function for different masses: the yellow curve represents $m=2 H$, the green curve $m=5 H$, the red curve $m=10 H$ and the purple curve $m=15 H$. The blue curve is the massless propagator given by the Hankel function with $\nu=3 / 2$.

The advantage of the propagator constructed from the hypergeometric function is that it exactly describes the free dynamics for a massive scalar field theory. Nevertheless the price to pay is that we have to deal with a function that was constructed numerically. In particular when we will consider the radiative corrections we will be confronted with the problem of renormalizing the integral of the propagator. The real issue here is how to properly disentangle the divergences from the finite contribution in a numerical integration. 


\section{WKB propagator}

In the following we will introduce the massive propagator in the semi-classical WKB approximation in order to get an analytic expression for the counter-terms. This is a crucial point in the renormalization procedure. Since the WKB propagator has the same UV behavior of the full massive propagator (4.39), one can exploit it in order to have an explicit expression for the massive counter-term. We will regularize the numerical integral by introducing an explicit cutoff on momentum space. Subsequently the cutoff dependence is removed by manually subtracting the analytic contribution of the WKB counter-terms.

For a very massive quantum field with a Bunch-Davies vacuum [135] as the initial state, a good solution of the free field equations is given by the WKB approximation. The Hadamard propagator can be defined from the field solution $V(k, \tau)$

$$
F\left(k, \tau_{1}, \tau_{2}\right)=\mathcal{R}\left[V\left(k, \tau_{1}\right) V^{*}\left(k, \tau_{2}\right)\right]
$$

Then in the WKB approximation $H / m \ll 1$ one can approximate $V$ as [140-142]

$$
V(k, \tau)=\frac{\exp \left[-i \int_{\tau_{\text {in }}}^{\tau} d \tau_{1} \sqrt{k^{2}+m^{2} a\left(\tau_{1}\right)^{2}}\right]}{\sqrt{2} a(\tau)\left(k^{2}+m^{2} a(\tau)^{2}\right)^{1 / 4}},
$$

and define the Hadamard propagator obtained in the WKB approximation

$$
F_{W K B}\left(k, \tau_{1}, \tau_{2}\right)=\frac{\cos \left[\int_{\tau_{1}}^{\tau_{2}} d \tau \sqrt{k^{2}+m^{2} a(\tau)^{2}}\right]}{2 a\left(\tau_{1}\right) a\left(\tau_{2}\right)\left(k^{2}+m^{2} a\left(\tau_{1}\right)^{2}\right)^{1 / 4}\left(k^{2}+m^{2} a\left(\tau_{2}\right)^{2}\right)^{1 / 4}}
$$

It should be noted that this propagator is suppressed by $m^{-1} a^{-3}\left(\tau_{1}\right)$ for equal time $\tau_{1}=\tau_{2}$ and for $k \ll m a\left(\tau_{1}\right)$ [29], while in the UV regime the dependence is weaker and proportional to $a^{-2}\left(\tau_{1}\right)$.

In Figure 4.3 we plotted both the Fourier transform of the full massive propagator on discrete points for large masses and we compared them with the propagator obtained in the WKB approximation. We see that the matching is almost perfect.

This means that the WKB propagator provides a very good approximation of the full massive propagator in eq. (4.39) in the UV regime and for masses $m \gg H$. In the following we will use the WKB approximation in order to get an explicit expression for the mass counter-term.

The equal-time two-point correlation function in the WKB approximation is given 


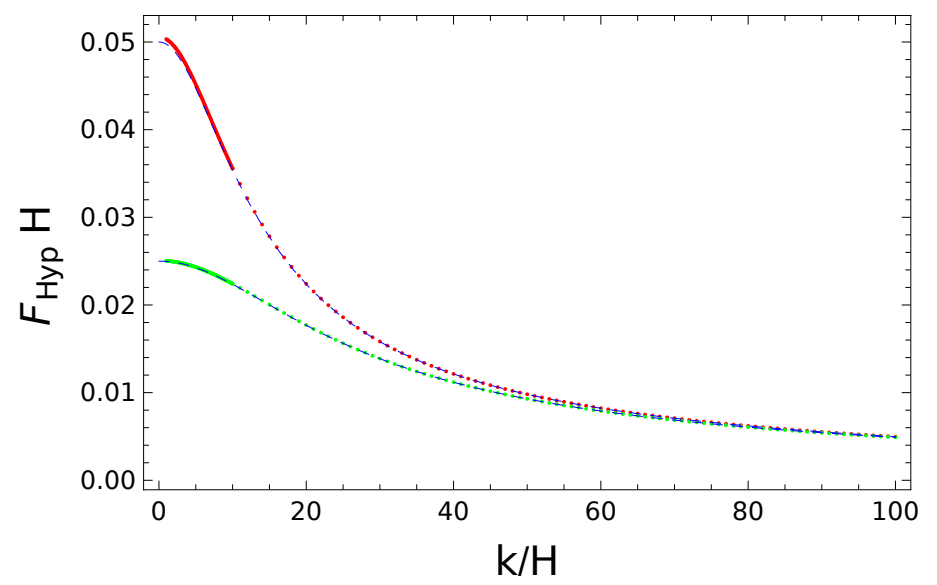

Figure 4.3: Comparison of the massive Hadamard propagator reconstructed from the hypergeometric function (4.39) (list of points) and the WKB propagator (4.46) (dashed blue lines) for a mass of $10 H$ (red points) and $20 H$ (green points).

by

$$
F_{\mathrm{WKB}}(k, \tau, \tau)=\frac{H^{2} \tau^{2}}{2 \sqrt{\frac{m^{2}}{H^{2} \tau^{2}}+k^{2}}},
$$

where $F$ is the usual Hadamard propagator.

This concludes our discussion about the Schwinger and Keldysh formalism for time-dependent setups where the time translational symmetry of the system is broken both because of the background and because of the explicit time dependence of the Hamiltonian. The main difference with respect to the in-out approach of quantum field theory is that in scattering processes the initial free vacuum states are given in the infinite past. In the closed-time-path formalism instead the time-evolution starts from the initial time where the system is supposed to be in an eigenstate of the free theory. Therefore in the in-out approach only one time-evolution operator $U(\infty,-\infty)$ appears, which is different from our case where the time-evolution is given by the two operators $U\left(-\infty, t_{\text {in }}\right)$ and $U^{\dagger}\left(\infty, t_{\text {in }}\right)$ that cannot be combined in eq. (4.8) because the system is not invariant under time translations. 


\section{CHAPTER 5}

\section{LaPTER RENORMALIZATION IN THE CLOSED-TIME-PATH FORMALISM}

In this chapter the renormalization procedure for quantum field theories in curved spacetime for time-dependent setups is explored. We will investigate in details the ultraviolet behavior of the two- and four-point function in Minkowski and de-Sitter backgrounds in the theoretical setting of the Schwinger and Keldysh framework (see [143] as a guideline of the techniques that we use).

Having as a final goal the study of radiative corrections to cosmological observables during the inflationary epoch, the quantum behavior of a simple scalar field theory with quartic self-interaction is investigated. The theory can be easily extended to a two scalar fields setup where the light field gets radiative corrections from virtual effects of the heavier field.

The time-evolution of observables is started at a finite initial time where the theory is assumed to be in the vacuum state of the free theory. As a consequence the system is out-of-equilibrium since it is not starting from an eigenstate of the interacting system.

In the closed-time-path formalism one tries to overcome the breaking of the Poincaré symmetry by splitting the four-vector $x^{\mu}$ into two components: the time and the spatial coordinates. Despite the fact that one cannot use the traditional perturbative approach of quantum field theory in Fourier space since the plane waves are no longer solutions of the Klein-Gordon operator, one can anyway exploit the symmetries of the spatial coordinates in order to obtain quantities that depend on time and on the 3-momentum. This approach, which is necessary because we do not have the full set of symmetries, has the advantage that it clarifies how divergences emerge from space and time.

In the Schwinger and Keldysh theoretical framework the time-translational symmetry is broken in two distinct ways. The first is a consequence of the formalism and 
is connected to the fact that the interacting theory starts at a finite initial time $t_{\text {in }}$. Using a different perspective one can interpret this non-locality as a time-dependent interaction in the Lagrangian proportional to the step-function $\theta\left(t-t_{\text {in }}\right)$. In this picture the system evolves according to the free theory before the initial time and according to the full theory after the interaction is switched-on. We will study the imprint of the initial time in Chapter 6, where the time evolution of the two-point function in Minkowski and de-Sitter background is analyzed, using different interaction profiles. The second property that affects the time dependence of observables is connected to the cosmological background that has an explicit time dependence in order to describe the time evolution of the universe. By dealing with renormalization in curved spacetime in the framework of the CTP formalism we therefore expect that observables will depend on time both because of the background and because of the finite initial time where the interaction starts.

In the first sections the one-loop corrections to the equal-time two-point function (Section 5.1) and four-point function (Section 5.2) are computed both in Minkoswski and de-Sitter spacetime. In Section 5.3, the massive counter-term in de-Sitter spacetime in the WKB approximation is computed. Finally, in Section 5.4 the counter-terms found in the CTP approach are compared to those obtained with the completely independent renormalization scheme of adiabatic renormalization.

\subsection{The equal-time two-point correlation function}

The first case that we will study is the renormalization of the equal-time two-point function. The only tree-level contribution is given by the Hadamard propagator $F(k, t, t)$ (see Figure 5.1). The other contributions $G^{R}(k, t, t)$ and $G^{A}(k, t, t)$ are identically vanishing for equal times. It is interesting to observe that even the one-loop corrections to these propagators are not contributing because of their causal properties ${ }^{1}$.

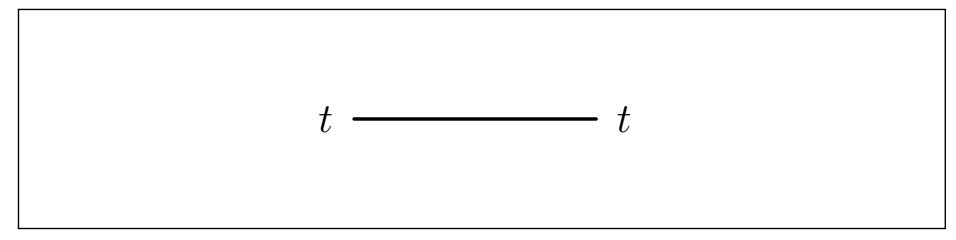

Figure 5.1: Tree-level equal-time two-point correlation function $F(k, t, t)$.

We will therefore proceed with the analysis of the one-loop corrections to the equal-time Hadamard two-point function since it is the only propagator that has a

\footnotetext{
${ }^{1}$ The advanced and retarded propagators are proportional to the step-function $\theta\left(t_{1}-t_{2}\right)$ that defines their causal properties. The product $G^{R}\left(k, t_{1}, t_{2}\right) G^{A}\left(k, t_{1}, t_{2}\right)$ vanishes.
} 
non-zero tree-level contribution and gets radiative corrections at one-loop level. In the following we will refer to the one-loop corrections as the tadpole contribution. We will start with the analysis of the tadpole in Minkowski spacetime in order to test the methods since the calculations can be easily performed analytically and compared with the results present in the literature. In the second part we will perform the same analysis in a more relevant background, i.e. de-Sitter spacetime, for a (quasi) massless scalar field.

\section{One-loop radiative corrections in Minkowski spacetime from a massless scalar field}

In the following, we will calculate the one-loop contributions to the two-point function for a massless scalar field theory with quartic coupling $\lambda \phi^{4}$. The theory is described in Chapter 4 and the Feynman rules are given in Appendix A.1.

Considering that the propagators $G^{R}$ and $G^{A}$ vanish for coinciding time, there is only one possible vertex (the one proportional to $\left(\phi^{(1)}\right)^{3} \phi^{(2)}$ ) for the one-loop corrections and therefore only the tadpole diagram in Figure 5.2 survives. One has to consider also the mirror diagrams where the only difference is the interchange of the two external propagators from $G^{R}\left(k, t, t_{1}\right) F\left(k, t_{1}, t\right)$ to $F\left(k, t, t_{1}\right) G^{A}\left(k, t_{1}, t\right)$. Because the $F$-propagator is symmetric under the exchange of the two time arguments and $G^{A}\left(k, t, t_{1}\right)=G^{R}\left(k, t_{1}, t\right)$ both diagrams give the same contribution.

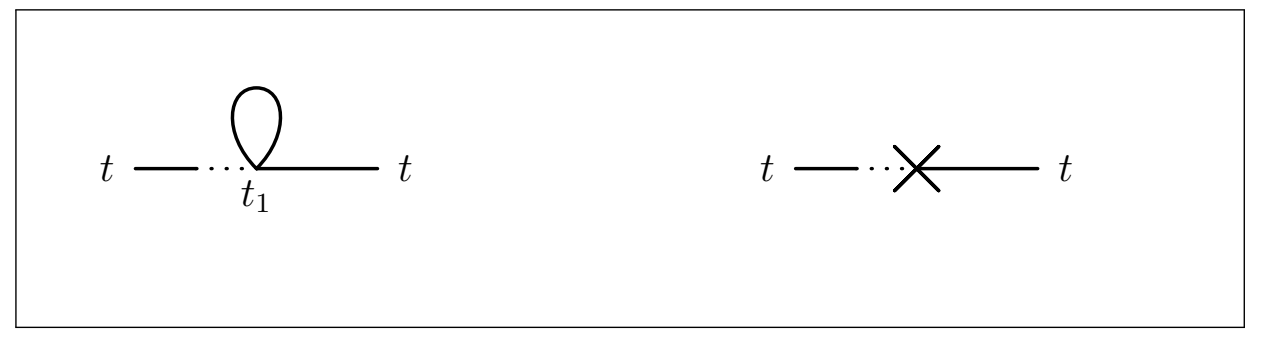

Figure 5.2: Tadpole diagram and its counter-term for a quartic self-interacting scalar field theory in Minkowski spacetime. The mirror diagrams should also be considered and give the same contributions.

The amplitude corresponding to the tadpole diagram of Figure 5.2 with an additional factor 2 in order to take into account the mirror diagram is

$$
\int_{t_{\mathrm{in}}}^{t} d t_{1}\left(-i G^{R}\left(k, t, t_{1}\right)\right) F\left(k, t_{1}, t\right)\left[2\left(\frac{-i \lambda}{2}\right) \int \frac{d p^{3}}{(2 \pi)^{3}} F\left(p, t_{1}, t_{1}\right)\right] \text {, }
$$

where $F$ and $G^{R}$ are the propagators of the theory in the Schwinger basis. For a massless scalar field theory in Minkowski background they are given by the massless 
limit of eq. (4.28). It should be noted that the square brackets, that we name $A_{\mathrm{amp}}$, is divergent in the ultraviolet regime and has to be renormalized in order to make the correlation function physically meaningful. By introducing an explicit UV cutoff $\Lambda$ we can solve the integral analytically

$$
A_{\mathrm{amp}}=\left(\frac{-i \lambda}{2 \pi^{2}}\right) \int_{0}^{\Lambda} d k \frac{k}{2}=\left(\frac{-i \lambda}{8 \pi^{2}}\right) \Lambda^{2} .
$$

We renormalize the amplitude by identifying and subtracting only the UV divergences from the regulated amplitude $A_{\text {amp }}$ with the definition of the following mass counter-term

$$
\delta m^{2}=\left(\frac{\lambda}{16 \pi^{2}}\right) \Lambda^{2}
$$

The result is in agreement with the results of dimensional regularization present in the literature $[84,85,144]$. We are now able to perform the time integral in order to take into account the contribution of the external propagators. A simple integration gives

$$
-i A_{\mathrm{amp}} \cdot \frac{\sin \left(k\left(t-t_{\mathrm{in}}\right)\right)^{2}}{2 k^{3}} .
$$

This term will not contribute to the final amplitude because it is completely canceled by the mass counter-term $\delta m^{2}$. In principle, we could use a different renormalization scheme and define a different counter-term $\delta \tilde{m}^{2}=\delta m^{2}+C$, where $C$ is a constant constrained to be independent on time because of the symmetry of the system and exploit the renormalization freedom in order to get a non-zero amplitude

$$
-C \cdot \frac{\sin \left(k\left(t-t_{\text {in }}\right)\right)^{2}}{2 k^{3}}
$$

From the last equation we observe that the only time dependence of the renormalized correlation function comes from the external propagators. Since we are working on a flat spacetime it is clear that the time dependence is a consequence of the finite initial time introduced in the Schwinger and Keldysh formalism. We will analyze and discuss the finite time contributions in Chapter 6 where we will compute the same quantity using different interaction profiles ${ }^{2}$.

\footnotetext{
${ }^{2}$ In the CTP formalism the interaction is switched-on exactly at the initial time $t_{\text {in }}$.
} 


\section{One-loop corrections in Minkowski spacetime from a massive scalar field}

In the next example, we consider the quantum corrections from a massive scalar field in Minkowski spacetime. This case generalizes our discussion about loop corrections from a massless scalar field where we found the mass-independent contribution to the UV counter-term $\delta m^{2}$, which is universal.

Let us consider a massive scalar field theory with quartic coupling. The propagators are given by eq. (4.28). The one-loop correction to the two-point correlation function is given by the tadpole in Figure 5.2 plus the mirror diagrams that give an additional factor 2

$$
\int_{t_{\mathrm{in}}}^{t} d t_{1}\left(-i G^{R}\left(w_{k}, t, t_{1}\right)\right) F\left(w_{k}, t_{1}, t\right)\left[2\left(\frac{-i \lambda}{2}\right) \int \frac{d p^{3}}{(2 \pi)^{3}} F\left(w_{p}, t_{1}, t_{1}\right)\right],
$$

where $F$ and $G^{R}$ are the massive propagators and the square brackets is the loop contribution that we name $A_{\mathrm{amp}}$. As before the loop integral is UV divergent and can be integrated analytically after having introduced an explicit cutoff $\Lambda$ in momentum space. We obtain

$$
\begin{aligned}
A_{\mathrm{amp}} & =\frac{-i \lambda}{8 \pi^{2}}\left(\Lambda^{2} \sqrt{1+\frac{m^{2}}{\Lambda^{2}}}-m^{2} \operatorname{arcsinh}\left(\frac{\Lambda}{m}\right)\right) \\
& =\frac{-i \lambda}{8 \pi^{2}}\left(\Lambda^{2}+\frac{1}{2} m^{2}+m^{2} \log \left(\frac{m}{2 \Lambda}\right)\right)+\mathcal{O}\left(\Lambda^{-2}\right) .
\end{aligned}
$$

This result is in agreement with the analogous in-out results in the literature [84]. In addition to the quadratic divergence that we found before in eq. (5.3), we have a logarithmic divergence proportional to the mass squared regulated by $\Lambda$. Moreover the limit for vanishing mass is finite and it is consistent with eq. (5.2). Indeed for the massless theory we already found that the loop integral is finite in the infrared regime.

By applying the minimal subtraction scheme in order to remove only the terms that depend on $\Lambda$, we found the finite amplitude

$$
\left[A_{\mathrm{amp}}\right]_{\mathrm{ren}}=\frac{-i \lambda m^{2}}{16 \pi^{2}}\left(1+\log \left(\frac{m^{2}}{4 \mu^{2}}\right)\right)
$$

where $\mu$ is the arbitrary renormalization scale. The massive counter-term for the chosen scheme is

$$
\delta m^{2}=\frac{\lambda}{16 \pi^{2}}\left(\Lambda^{2}-m^{2} \log \left(\frac{\Lambda}{\mu}\right)\right)
$$

and in the limit for vanishing mass we recover the massless counterterm (5.3). 
Unlike the massless case where we had to consider a different renormalization scheme in order to have a non-zero contribution, here the renormalized amplitude is non vanishing because of the finite terms and because of the residual contribution from the logarithmic divergence. Even in this case the full two-point correlation function can be integrated analytically. By considering the full integral with the contribution from the external propagators we found the final amplitude

$$
-i\left[A_{\mathrm{amp}}\right]_{\mathrm{ren}} \cdot \frac{\sin \left(\sqrt{k^{2}+m^{2}}\left(t-t_{\mathrm{in}}\right)\right)^{2}}{2\left(k^{2}+m^{2}\right)^{3 / 2}} .
$$

Because we are dealing with renormalization on flat spacetime we can interpret the time dependence as a consequence of the time-evolution of the interacting theory from the initial time $t_{\text {in }}$, where we have set abruptly the initial Minkowski vacuum state. Again in the limit for vanishing mass we consistently recover the massless result (5.5).

To conclude the discussion, it should be noted that since we are on a flat spacetime we expect that in the adiabatic limit, i.e. in the limit $t-t_{\text {in }} \rightarrow \infty$, the dependence on the initial time should disappear. It is clearly not the case because the limit

$$
\lim _{t_{\text {in }} \rightarrow \infty} \sin \left(t-t_{\text {in }}\right)^{2}
$$

does not exit. Nevertheless the oscillatory factor can be treated in the language of distribution theory. We will investigate this issue more in details at the end of Chapter 6.

\section{One-loop corrections in quasi de-Sitter space from a (nearly) massless field}

We will now consider a background of cosmological interest and apply the in-in formalism to the computation of the two-point correlation function for a scalar field in quasi de-Sitter spacetime. At tree-level only the Hadamard propagator $F$ is non vanishing and has radiative one-loop corrections. For a massless scalar field theory in de-Sitter the equal-time propagator is given by eq. (4.36). The limit of the propagator for a small mass and small momenta is given in eq. (4.38). Let us consider the oneloop radiative corrections in the case of a (almost) massless scalar field in de-Sitter spacetime. We will use the theory described in Section 4 and the Feynman rules given in Section A.1.

As in the case of a Minkowski spacetime, only the Hadamard two-point function gets radiative corrections and they are given in Figure 5.3 plus the same contributions from the mirror diagrams. 


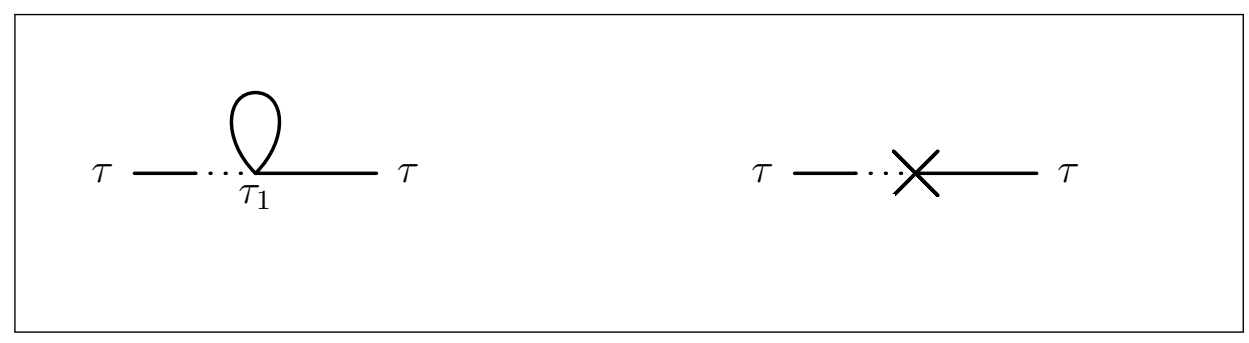

Figure 5.3: Tadpole diagram and its counter-term for a quartic self-interacting scalar field theory in de-Sitter spacetime. The mirror diagrams should also be considered and give the same contributions.

The corresponding integral of the total amplitude reads

$$
\int_{\tau_{\mathrm{in}}}^{\tau} d \tau_{1}\left(-i G^{R}\left(k, \tau, \tau_{1}\right)\right) F\left(k, \tau_{1}, \tau\right)\left[2\left(\frac{-i \lambda}{2} a^{4}\left(\tau_{1}\right)\right) \int \frac{d p^{3}}{(2 \pi)^{3}} F\left(p, \tau_{1}, \tau_{1}\right)\right],
$$

where $F$ and $G^{R}$ are the de-Sitter Hadamard and retarded propagators in the closedtime-path formalism and the additional factor 2 is inserted in order to take into account the mirror diagrams. On a de-Sitter background for a massless scalar field theory the propagators are given by eq. (4.34).

The loop integral can be solved analytically after the introduction of a cutoff in momentum space $\Lambda a\left(\tau_{1}\right)$ [32]. We remark that the scale factor dependence is important here because $\Lambda$ is a physical cutoff and regulate integrals over physical momenta $k_{\text {phys }}$. Since we are considering loop integrals over comoving momenta $k=k_{\text {phys }} a(\tau)$, the cutoff assumes the form $\Lambda a(t)$.

The loop integral is solved analytically. First we introduce an arbitrary mass scale $M$ and we split the integral in two parts

$$
\int \frac{d p^{3}}{(2 \pi)^{3}} F\left(p, \tau_{1}, \tau_{1}\right)=\left[\int_{0}^{M}+\int_{M}^{\infty}\right] \frac{d p}{2 \pi^{2}} p^{2} F\left(p, \tau_{1}, \tau_{1}\right) .
$$

In the first integral we used the asymptotic expansion for the propagator in eq. (4.38)

$$
\begin{aligned}
\int_{0}^{M} \frac{d p}{2 \pi^{2}} p^{2} F\left(p, \tau_{1}, \tau_{1}\right) & =\frac{1}{2 \pi^{2}} \int_{0}^{M} d p \frac{H^{2}}{2 p}\left(p^{2} \tau_{1}^{2}\right)^{\varepsilon} \\
& =\frac{H^{2}\left(M^{2} \tau_{1}^{2}\right)^{\varepsilon}}{8 \pi^{2} \varepsilon} \\
& =\frac{H^{2}}{8 \pi^{2}}\left(\frac{1}{\varepsilon}+\log \left(M^{2} \tau_{1}^{2}\right)\right)+\mathcal{O}(\varepsilon) .
\end{aligned}
$$

In the last line we used the Laurent expansion in $\varepsilon$ to extract the singular behavior of the IR-cutoff. It should be noted that in this case the loop integral contains an 
IR-divergence for massless fields, which is not present for a flat background. The second integral can be regularized in the UV regime by introducing a physical cutoff $\Lambda$

$$
\begin{aligned}
\int_{M}^{\Lambda a\left(\tau_{1}\right)} \frac{d p}{2 \pi^{2}} p^{2} F\left(p, \tau_{1}, \tau_{1}\right) & =\frac{1}{2 \pi^{2}} \int_{0}^{\Lambda a\left(\tau_{1}\right)} d p \frac{H^{2}}{2 p}\left(1+p^{2} \tau_{1}^{2}\right) \\
& =\frac{H^{2}}{8 \pi^{2}}\left(\tau_{1}^{2}\left(\Lambda^{2} a^{2}\left(\tau_{1}\right)-M^{2}\right)+2 \log \left(\frac{\Lambda a\left(\tau_{1}\right)}{M}\right)\right) .
\end{aligned}
$$

The total integral is finally given by

$$
\begin{aligned}
{\left[\int \frac{d p^{3}}{(2 \pi)^{3}} F\left(p, \tau_{1}, \tau_{1}\right)\right]_{\mathrm{reg}} } & =\frac{H^{2}}{8 \pi^{2}}\left(\frac{1}{\varepsilon}+\log \left(M^{2} \tau_{1}^{2}\right)+\tau_{1}^{2}\left(\Lambda^{2} a^{2}\left(\tau_{1}\right)-M^{2}\right)+2 \log \left(\frac{\Lambda a\left(\tau_{1}\right)}{M}\right)\right) \\
& =\frac{H^{2}}{8 \pi^{2}}\left(\frac{1}{\varepsilon}+2 \log \left(\frac{\Lambda}{H}\right)+\left(\frac{\Lambda}{H}\right)^{2}\right)
\end{aligned}
$$

where we sent the mass scale $M$ to 0 because it is arbitrary and the limit is well-defined. This gives us directly the amputated two-point correlation function

$$
\left[A_{\mathrm{amp}}\right]_{\mathrm{reg}}=-i \frac{\lambda a\left(\tau_{1}\right)^{4} H^{2}}{8 \pi^{2}}\left(\frac{1}{\varepsilon}+2 \log \left(\frac{\Lambda}{H}\right)+\left(\frac{\Lambda}{H}\right)^{2}\right)
$$

The amplitude can be renormalized in the minimal subtraction scheme by identifying and subtracting the UV-divergences from the regulated expression. This requires the definition of the mass and curvature counter-terms

$$
\begin{aligned}
\delta m^{2} & =\frac{\lambda}{16 \pi^{2}} \Lambda^{2} \\
\delta \xi & =\frac{\lambda}{8 \pi^{2}} \frac{1}{12} \log \left(\frac{\Lambda}{\mu}\right), \text { for the case } \xi=0 .
\end{aligned}
$$

We remark that the expressions (5.18) and (5.19) seem to have a different normalization. The reason is that eq. (5.18) is the total amputated amplitude which takes into account also the contribution of the mirror diagram. To regulate the full contribution one has to consider also the mirror mass counter-term that gives exactly the same cancellation and provides the additional factor 2 .

The regulated amplitude has the same quadratic and logarithmic divergences that we found Minkowski spacetime. This is expected because it has been proven in the algebraic QFT framework that the UV divergences in curved spacetime are of the same order as in Minkowski spacetime, which means that the type and the number of the counter-terms is the same $[111,112,115]$. Because in the FRW spacetime the curvature is given by $\mathcal{R}(\tau)=12 H^{2}(\tau)$, we can interpret the logarithmic contribution 
proportional to $H^{2}$ in eq. (5.18) as a covariant correction of the curvature term. Both the mass counter-term and the curvature counter-term are in agreement with similar results in the literature using dimensional regularization [33] and the effective action method [34]. Moreover the counter-terms are covariant expressions of geometrical quantities in agreement with [145] and they can be reabsorbed into the redefinition of the wave function, mass, and coupling constant parameters in the Lagrangian.

After the subtraction of the divergences we obtain the renormalized amputated amplitude

$$
\left[A_{\mathrm{amp}}\right]_{\mathrm{ren}}=-i \frac{\lambda a\left(\tau_{1}\right)^{4} H^{2}\left(\tau_{1}\right)}{8 \pi^{2}}\left(\frac{1}{\varepsilon}-2 \log \left(\frac{H\left(\tau_{1}\right)}{\mu}\right)\right) .
$$

The result has a logarithmic dependence on $H\left(\tau_{1}\right)$ which is consistent with the discussion on loop corrections of [32]. This dependence comes from the scale factor of the comoving cutoff $\Lambda a(t)$. With the improper definition of the cutoff without the scale factor correction we would have found a logarithmic dependence $\log (\mathrm{Ha} / \mu)$. The scale factor dependence of $a\left(\tau_{1}\right)^{4}$ is related to the definition of the vertices and will be compensated when considering the external legs.

Now we can finally include the contribution of the external propagators as in eq. (5.13) in order to have the full loop correction to the two-point correlation function. The integral can be performed analytically using the massless scalar field propagators (4.34). The result is

$$
\begin{aligned}
\frac{-i}{6 k^{3}}\left[A_{\text {amp }} a\left(\tau_{1}\right)^{-4}\right]_{\text {ren }} \times & {\left[2+\left(\operatorname{Ci}(2 k \tau)-\operatorname{Ci}\left(2 k \tau_{\text {in }}\right)\right)\left(\left(-1+k^{2} \tau^{2}\right) \cos (2 k \tau)-2 k \tau \sin (2 k \tau)\right)\right.} \\
& +\left(\operatorname{Si}(2 k \tau)-\operatorname{Si}\left(2 k \tau_{\text {in }}\right)\right)\left(2 k \tau \cos (2 k \tau)+\left(-1+k^{2} \tau^{2}\right) \sin (2 k \tau)\right) \\
& +\frac{1}{2 k^{3} \tau_{\text {in }}^{3}}\left(4 k^{2} \tau_{\text {in }} \tau+\left(1+k^{2} \tau_{\text {in }}^{2}\right)\left(1-k^{2} \tau^{2}\right)\right) \sin \left(2 k\left(\tau-\tau_{\text {in }}\right)\right) \\
& \left.+\frac{1}{2 k^{3} \tau_{\text {in }}^{3}}\left(2 k \tau_{\text {in }}\left(1-k^{2} \tau^{2}\right)-2 k \tau\left(1+k^{2} \tau_{\text {in }}^{2}\right)\right) \cos \left(2 k\left(\tau-\tau_{\text {in }}\right)\right)\right],
\end{aligned}
$$

where $\mathrm{Ci}$ and $\mathrm{Si}$ are the sine and cosine integral (see Figure 5.4) defined as

$$
\begin{aligned}
& \operatorname{Ci}(x)=-\int_{x}^{\infty} d t \frac{\cos t}{t} \\
& \operatorname{Si}(x)=\int_{0}^{x} d t \frac{\sin t}{t}
\end{aligned}
$$

It should be noted that in eq. (5.22) the argument of the cosine integral is negative 


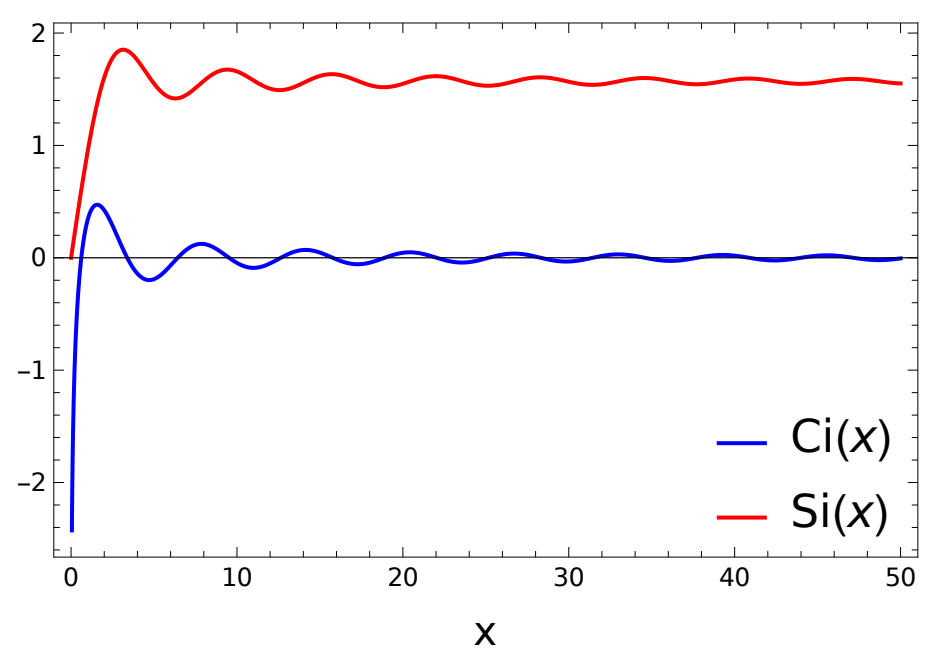

Figure 5.4: Cosine integral (blue line) and sine integral (red line) given in eq. (5.23). The cosine integral is defined only for positive numbers and has a logarithmic behavior about the origin. The sine integral is an even function and is well defined for all real numbers.

and therefore not well defined. Nevertheless the difference $\mathrm{Ci}(2 k \tau)-\mathrm{Ci}\left(2 k \tau_{\text {in }}\right)$ is well-behaved. Alternately one can consider the analytic extension to the complex plane.

In eq. (5.22) we observe a periodic behavior that has its largest impact in the early times. The oscillations are suppressed for large times where the logarithmic behavior dominates. We point-out that for larger elapsed time $\tau-\tau_{\text {in }}$ when the oscillations are damped-out and before the logarithmic regime, the dominant contribution is given by a constant value independent from $\tau_{\text {in }}$. We will elaborate more about the constant contribution of the two-point correlation function in Chapter 6.

At lowest order in $k \tau$ the solution simplifies to

$$
\frac{\lambda H^{2}(\tau)}{4 \pi^{2} 12 k^{3}}\left[\left(\frac{1}{\varepsilon}-2 \log \left(\frac{H}{\mu}\right)\right)\left(\log \left(\frac{\tau}{\tau_{\text {in }}}\right)+\frac{1}{3}-\frac{\tau^{3}}{3 \tau_{\text {in }}^{3}}\right)\right],
$$

where $\varepsilon$ is the IR-regulator and $\mu$ is an arbitrary renormalization energy scale. In eq. (5.24) we observe a logarithmic and polynomial dependence on the conformal time. The logarithmic divergence for small conformal times (that corresponds to an infinite coordinate time $t$ ) is a true divergence and it is present also in the full expression (as one can see in Figure 5.5). The secular terms in $\tau$ are an artifact that we are approximating an oscillatory function with a polynomial Taylor expansion for small $k \tau$.

The full solution is shown in Figure 5.5 for three different initial conditions in order to show the dependence on the initial time. One recognizes the logarithmic behavior at 


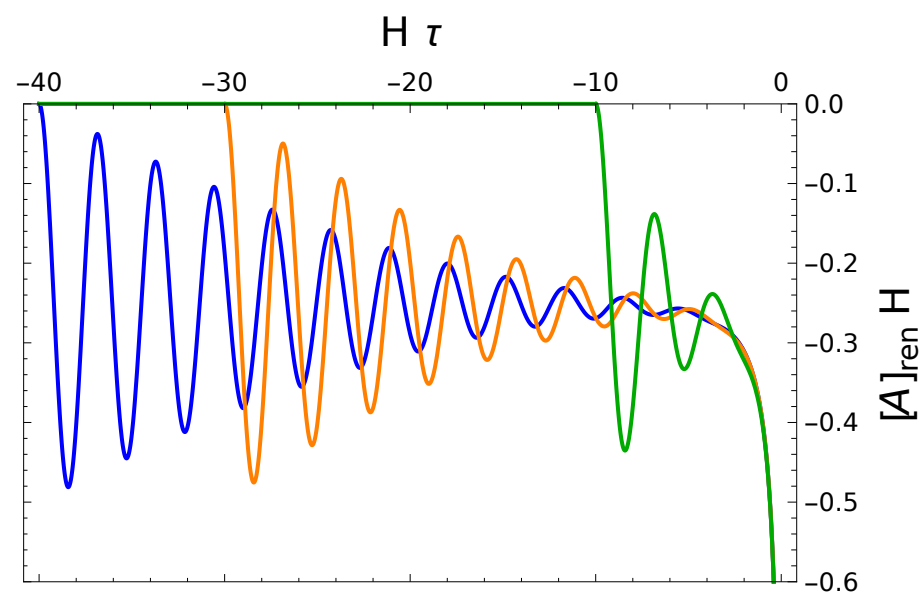

Figure 5.5: Time dependence of the renormalized two-point correlation function in de-Sitter spacetime for $H \tau_{\text {in }}=-40$ (blue curve), $H \tau_{\text {in }}=-30$ (orange curve), and $H \tau_{\text {in }}=-10$ (green curve) with $k / H=1, \varepsilon=1 /\left(16 \pi^{2}\right), H / \mu=1$ and $\lambda=1$.

late time (about $\tau=0$ ) and the spectral oscillations connected to the initial state, as in the Minkowski case. We will discuss more in details the origin of these oscillations in Chapter 6 where we discuss the interaction profile dependence of our result. As in Minkowski spacetime we cannot simply take the limit of $\tau_{\text {in }} \rightarrow-\infty$ because it is not well-defined. Moreover we decided to set the initial conditions to the Bunch-Davies vacuum $[20,135]$ at the beginning of inflation.

This concludes our discussion of the two-point function renormalization in Minkowski and de-Sitter spacetime using the closed-time-path formalism. In Chapter 7 we will discuss the physical consequences of our results and we will give our predictions to the primordial spectrum.

\subsection{The equal-time four-point correlation function}

We will now proceed with the investigation of the renormalization of the equal-time four-point function for different backgrounds. Higher order correlation functions are important quantities because they describe non-Gaussian features of the scalar field. In particular the four-point function is related to the connected trispectrum $\mathcal{T}_{\mathcal{R}}$ which is defined as $[42,43]$

$$
\left\langle\delta \phi_{\vec{k}_{1}} \delta \phi_{\vec{k}_{2}} \delta \phi_{\vec{k}_{3}} \delta \phi_{\vec{k}_{4}}\right\rangle_{c}=\frac{\dot{\phi}^{4}}{H^{4}}(2 \pi)^{3} \delta\left(\sum_{i} \vec{k}_{i}\right) \mathcal{T}_{\mathcal{R}}\left(\vec{k}_{1}, \vec{k}_{2}, \vec{k}_{3}, \vec{k}_{4}\right)
$$


We remark that the three-point function $\left\langle\delta \phi_{k_{1}} \delta \phi_{k_{2}} \delta \phi_{k_{3}}\right\rangle$ and all the other odd correlation functions are identically zero at first order because in our models the system is invariant under the field transformation $\phi \rightarrow-\phi$.

We will now apply the closed-time-path formalism and investigate the timedependence of the tree-level and radiative corrections to the equal-time four-point function with the same techniques used in the previous section.

First we will present our results for the tree-level contributions for a massive scalar field in Minkowski spacetime where the time dependence necessarily arises from the finite time of the initial conditions. Subsequently we will perform the same analysis for a massless scalar field in quasi de-Sitter spacetime where the time dependence arises both from the background and from the finite time. In the second part of the chapter we will investigate one-loop radiative corrections both in Minkowski and de-Sitter spacetime with analogous considerations about the time dependence.

\section{Tree-level contributions in Minkowski spacetime from a mas- sive scalar field}

We consider here the tree-level contributions to the four-point function from a massive scalar field in Minkowski spacetime. Only two diagrams $T_{1}$ and $T_{2}$ are contributing to the four-point correlation function and they are listed in Table 5.1, where the propagators are given by eq. (4.28). We start our calculations with the analysis of the tree-level in Minkowski spacetime in order to study the properties of the time dependence arising from the initial time $t_{\text {in }}$. The integrals assume a simple form only in

Table 5.1: Inequivalent Feynman diagrams for the tree-level contributions to the four-point function from a massive scalar field in Minkowski spacetime where $w_{k_{i}}=\sqrt{k_{i}^{2}+m^{2}}, k_{i}$ are the external momenta, $t$ is the external time, and $t_{\text {in }}$ is the initial time.

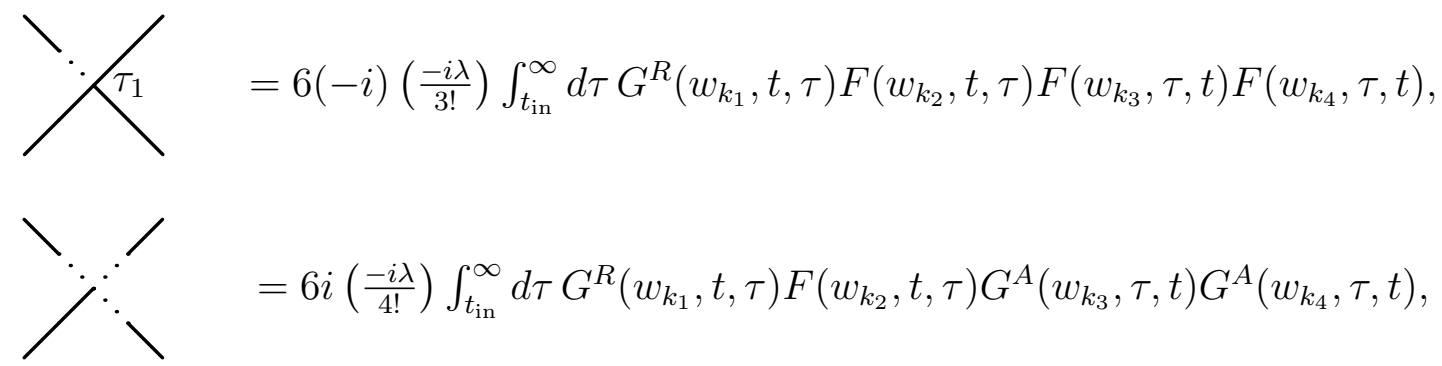

the limit of coinciding momenta $\left\{k_{i}\right\}_{i=1}^{4}=k$. Since we are interested in the properties of the time dependence we will consider only this limit. After the inclusion of all possible contributions coming from the permutation of the external propagators we 
found the analytic expression for the two diagrams

$$
\begin{aligned}
& T_{1}=-\frac{\lambda}{8\left(\sqrt{k^{2}+m^{2}}\right)^{5}}\left(1-\cos \left(\sqrt{k^{2}+m^{2}}\left(t-t_{\text {in }}\right)\right)^{4}\right), \\
& T_{2}=\frac{\lambda}{8\left(\sqrt{k^{2}+m^{2}}\right)^{5}} \sin \left(\sqrt{k^{2}+m^{2}}\left(t-t_{\text {in }}\right)\right)^{4},
\end{aligned}
$$

where $k$ is the external momentum and $t-t_{\text {in }}$ is the elapsed time. The total contribution assumes the simple form of a periodic function that is well-defined in the limit of a vanishing mass

$$
T=T_{1}+T_{2}=-\frac{\lambda}{16\left(\sqrt{k^{2}+m^{2}}\right)^{5}} \sin \left(2 \sqrt{k^{2}+m^{2}}\left(t-t_{\text {in }}\right)\right)^{2} .
$$

In Figure 5.6 we show the tree-level contributions to the four-point function from a massive scalar field. We observe that the time dependence is a consequence of the time integral of the external propagators as for the two-point function. Since we are working on a flat spacetime we do not expect any contribution from the background. This time-dependence is not present in the in-out approach because of the different picture. In the Schwinger and Keldysh formalism we consider the time evolution from an initial state where we assume the system to be in the free vacuum of the theory. As for the two-point function in the adiabatic limit the Poincaré symmetry is recovered.

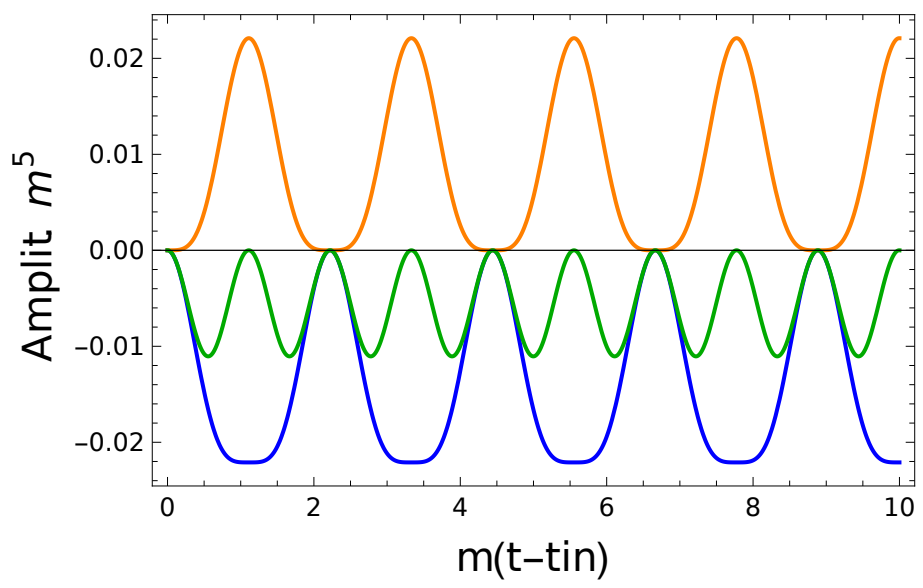

Figure 5.6: Tree-level contributions $T_{1}$ (blue line), $T_{2}$ (yellow line) and $T_{1}+T_{2}$ (green line) from the massive propagators in Minkowski spacetime for $k / m=1$ and $\lambda=1$. 


\section{Tree-level contributions in de-Sitter spacetime from a (nearly) massless scalar field}

We will now move to the discussion of the tree-level contribution on a background of cosmological interest during the inflationary epoch, i.e. quasi de-Sitter spacetime. We will investigate the tree-level contributions in de-Sitter spacetime from a (nearly) massless scalar field for a quartic interaction term. We will use the de-Sitter massless propagators defined in eqs. (5.15) since they are a good approximation for all superhorizon scales.

At tree-level only two finite diagrams $T_{1}$ and $T_{2}$ contribute to the four-point correlation function and they are listed in Table 5.2.

Table 5.2: Inequivalent Feynman diagrams for the tree-level contributions to the four-point function from a (nearly) massless scalar field in de-Sitter spacetime where $k_{i}$ are the external momenta, $\tau$ is the external conformal time, and $\tau_{\text {in }}$ is the initial time.

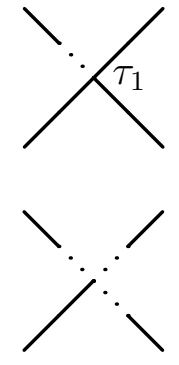

$$
\begin{aligned}
& =6(-i)\left(\frac{-i \lambda}{3 !}\right) \int_{\tau_{\mathrm{in}}}^{\infty} d \tau_{1} a^{4}\left(\tau_{1}\right) G^{R}\left(k_{1}, \tau, \tau_{1}\right) F\left(k_{2}, \tau, \tau_{1}\right) F\left(k_{3}, \tau_{1}, \tau\right) F\left(k_{4}, \tau_{1}, \tau\right), \\
& =6 i\left(\frac{-i \lambda}{4 !}\right) \int_{\tau_{\mathrm{in}}}^{\infty} d \tau_{1} a^{4}\left(\tau_{1}\right) G^{R}\left(k_{1}, \tau, \tau_{1}\right) F\left(k_{2}, \tau, \tau_{1}\right) G^{A}\left(k_{3}, \tau_{1}, \tau\right) G^{A}\left(k_{4}, \tau_{1}, \tau\right),
\end{aligned}
$$

The integrals cannot be computed analytically using the full propagators. We will therefore show the analytic results where we assumed the external propagators $F$ and $G^{R}$ to be in a super-Hubble regime, i.e. taking the first Taylor order in the limit of small $\left|k \tau_{i}\right|$. The full result is shown in Figure 5.7.

By considering all possible permutations of the external momenta we found

$$
\begin{aligned}
& T_{1}=-\lambda \frac{H^{4}\left(k_{1}^{3}+k_{2}^{3}+k_{3}^{3}+k_{4}^{3}\right)\left(\frac{\tau^{3}}{\tau_{\text {in }}^{3}}+3 \log \left(\frac{\tau_{\text {in }}}{\tau}\right)-1\right)}{72 k_{1}^{3} k_{2}^{3} k_{3}^{3} k_{4}^{3}}, \\
& T_{2}=\lambda \frac{H^{4}\left(k_{2}^{3} k_{3}^{3} k_{4}^{3}+k_{3}^{3} k_{4}^{3} k_{1}^{3}+k_{4}^{3} k_{1}^{3} k_{2}^{3}+k_{1}^{3} k_{2}^{3} k_{3}^{3}\right)\left(2 \frac{\tau^{9}}{\tau_{\text {in }}^{3}}+3 \tau^{6}-6 \tau^{3} \tau_{\text {in }}^{3}+\tau_{\text {in }}^{6}+18 \tau^{6} \log \left(\frac{\tau_{\text {in }}}{\tau}\right)\right)}{1296 k_{1}^{3} k_{2}^{3} k_{3}^{3} k_{4}^{3}} .
\end{aligned}
$$

We observe a logarithmic dependence on the conformal time that was present in the analogous calculation for the two-point function. We remark that the logarithmic dependence is not present in the Minkowski tree-level contribution and it is therefore a true time dependence coming from the background. The secular terms in $\frac{\tau}{\tau_{\text {in }}}$ are the first-orders of the early time oscillations visible in Figure 5.7. These oscillations (and as 


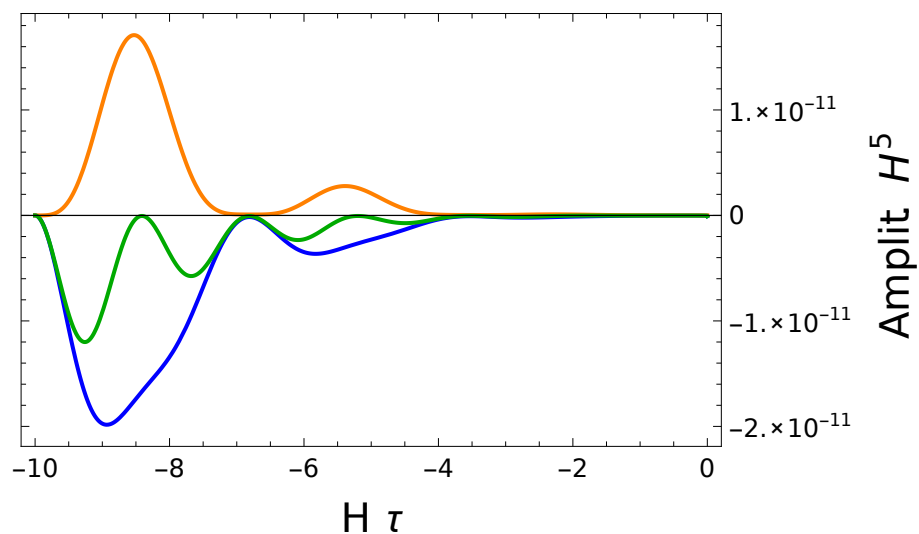

Figure 5.7: Tree-level contributions $T_{1}$ (blue line), $T_{2}$ (yellow line) and $T_{1}+T_{2}$ (green line) in de-Sitter spacetime from the massless propagators for $k_{1,2,3,4} / H=1, \lambda=10^{-13}$ and $H \tau_{\text {in }}=-10$.

a consequence also the secular terms) are a consequence of the initial conditions that we set in our theoretical framework. We point-out that the logarithmic dependence $\log (\tau)$ of $T_{1}$ is not visible in the plot.

We found that the trispectrum for a simple scalar field theory with quartic selfinteraction has a non-trivial contribution already at the tree-level. We remark that the size of the oscillation is proportional to the amplitude of the coupling constant $\lambda$.

This concludes our analysis of the tree-level contributions in the closed-time-path formalism for different backgrounds. In Section 7.5 we will investigate the cosmological implications and give our prediction for the cosmological parameter $\tau_{\mathrm{NL}}$ which is an observable quantity directly connected to the non-Gaussian features in the primordial universe.

\section{One-loop corrections in Minkowski spacetime from a massless scalar field}

We are going to analyze the one-loop radiative corrections to the four-point correlation function in Minkowski spacetime from a massless scalar field with quartic self-coupling in order to understand how divergences arise in the closed-time-path formalism. We will then define the ultraviolet counter-term $\delta \lambda$ which is universal because the UV divergences in curved spacetime are of the same type and degree as in Minkowski spacetime.

In Section 3.4 the singular structure of the propagators is shown. For the considered case the Hörmander Criterion (see the Corollary 3.4.2.1 for the details) is not satisfied for the products $F G^{R}, F G^{A}, F^{2},\left(G^{R}\right)^{2}$, and $\left(G^{A}\right)^{2}$. We therefore expect to get new divergences for all of these products. In fact this will not be the case because as we 
will show in the next section with the explicit calculation in de-Sitter spacetime, the sum $\left(G^{A}\right)^{2}+\left(G^{R}\right)^{2}-F^{2}$ is finite. On the other hand the singular product $F G^{R}$ will give rise to a true divergence.

As a consequence only two diagrams give new divergences and have to be renormalized. Here we are only interested in the divergent part and we will consider only the divergence coming from the two diagrams in Figure 5.8. The mirror diagrams should also be considered and they are given by the interchange of the two virtual times $\tau_{1}$ and $\tau_{2}$. The other possible contractions give finite contributions or vanish because of the causality restrictions of $G^{R}$.

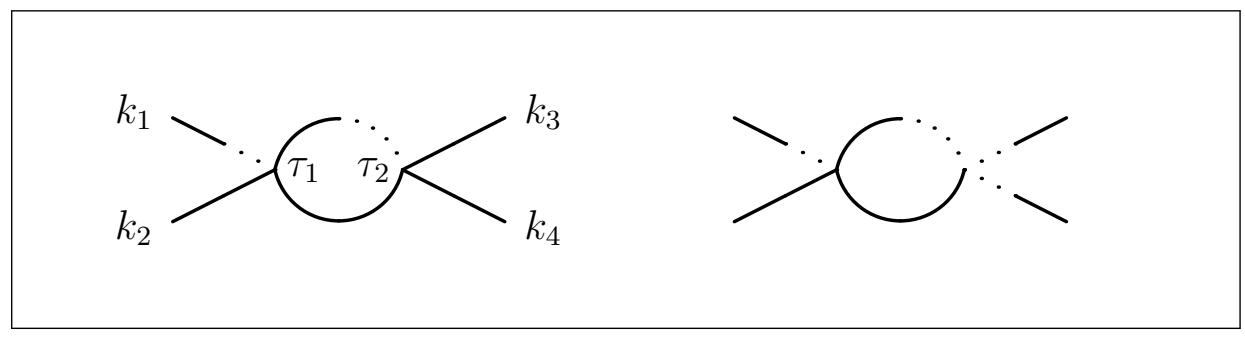

Figure 5.8: Fish diagrams $A_{1}$ (left) and $A_{2}$ (right). In addition one has to consider also the mirror diagrams.

In the following, we show our calculations for the amputated amplitude only for diagram $A_{1}$. The other amplitude will involve the same loop integral with a different normalization due to the different vertices. To get the expression for $A_{2}$ it is enough to perform the following replacement

$$
\left(\frac{-i \lambda}{3 !}\right)^{2} \rightarrow\left(\frac{-i \lambda}{3 !}\right)\left(\frac{-i \lambda}{4 !}\right)
$$

The counter-term $\delta \lambda$ that will be defined in order to cancel the divergence of $A_{1}$ will also cancel the divergence of $A_{2}$ and of mirror diagrams. The amputated amplitude of diagram $A_{1}$ is given by

$$
(-i)\left(\frac{-i \lambda}{3 !}\right)^{2} \int \frac{d^{3} p_{1}}{(2 \pi)^{3}} \int \frac{d^{3} p_{2}}{(2 \pi)^{3}} F\left(p_{1}, t_{1}, t_{2}\right) G^{R}\left(p_{2}, t_{1}, t_{2}\right)(2 \pi)^{3} \delta\left(k_{1}+k_{2}-p_{1}-p_{2}\right)
$$

Using the massless propagators in Minkowski spacetime given by (4.28) in the limit $m \rightarrow 0$, we are able to find the amputated amplitude

$$
\frac{i \lambda^{2} \theta\left(t_{1}-t_{2}\right) \sin \left(k\left(t_{1}-t_{2}\right)\right) \sin ^{2}\left(\left(t_{1}-t_{2}\right) \Lambda\right)^{2}}{8 \pi^{2} k\left(t_{1}-t_{2}\right)}
$$

In the limit of small external momenta, we can neglect higher orders in $\left|k\left(t_{1}-t_{2}\right)\right|$ 
and get

$$
\left(\frac{i \lambda^{2} \pi}{(2 \pi)^{3}}\right) \frac{\theta\left(t_{1}-t_{2}\right) \sin ^{2}\left(\left(t_{1}-t_{2}\right) \Lambda\right)}{\left(t_{1}-t_{2}\right)} .
$$

In order to understand the singular behavior of $\sin ^{2}\left(\left(t_{1}-t_{2}\right) \Lambda\right)$, one has to study the previous expression in the language of distributions. In [143] it has been proven that the following equality holds

$$
\theta(\Delta \tau) \frac{\sin ^{2}(\Lambda \Delta \tau)}{\Delta \tau}=\frac{1}{2}\left(\frac{\theta(-\eta+\Delta \tau)}{\Delta \tau}+\delta(\Delta \tau)(\gamma+\log (2 \Lambda \eta))\right)
$$

where $\eta$ is a time regulator that will be sent to zero at the end. The amputated fish diagram reads

$$
\left(\frac{i \lambda^{2} \pi}{2(2 \pi)^{3}}\right)\left(\frac{\theta\left(-\eta+\left(t_{1}-t_{2}\right)\right)}{\left(t_{1}-t_{2}\right)}+\delta\left(t_{1}-t_{2}\right)(\gamma+\log (2 \Lambda \eta))\right)
$$

The Lagrangian counter-term $\delta \lambda$ can be identified in the minimal subtraction scheme by subtracting only the divergent contributions from the regulated expression. One finds directly that

$$
-3\left(\frac{i \pi \lambda^{2}}{2(2 \pi)^{3}}\right) \log \left(\frac{\Lambda}{\mu}\right)=: i \delta \lambda
$$

where the additional factor 3 comes from the contribution of different channels $s, t$, and $u$.

The renormalized amputated amplitude for the fish diagram simplifies to

$$
\frac{i \pi \lambda^{2}}{2(2 \pi)^{3}}\left(\frac{\theta\left(-\eta+\left(t_{1}-t_{2}\right)\right)}{\left(t_{1}-t_{2}\right)}+\delta\left(t_{1}-t_{2}\right)(\gamma+\log (2 \mu \eta))\right) \text {. }
$$

The result is in agreement with the results in the literature of dimensional regularization $[84,85,144]$. Moreover, as observed in the radiative corrections of the two-point function, we do not have any explicit time dependence in the amputated amplitude. The non-local dependence on the elapsed time $t-t_{\text {in }}$ will arise when considering the effect of the external propagators.

\section{One-loop corrections in de-Sitter spacetime from a (nearly) massless scalar field}

In the following section we will consider the one-loop radiative corrections to the four-point function on a quasi de-Sitter background in the theoretical framework of the Schwinger and Keldysh formalism. As observed in Minkowski spacetime, this com- 
putation involves the singular product of two propagators and has to be renormalized. All the inequivalent contributions are listed in Table 5.3.

Table 5.3: Inequivalent Feynman diagrams for the one-loop correction to the four-point function. In the last column $\tau_{1}$ and $\tau_{2}$ denote the time of the left and right vertex and $\tau$ is the external time.

\begin{tabular}{|c|c|c|c|c|c|}
\hline Id & Coeff & Graph & Loop & Ext. contrib. & Time constraints \\
\hline$A_{1}$ & 36 & & $-i G^{R} F$ & $-i G^{R} F F F$ & $\tau_{2}<\tau_{1}, \tau_{1}<\tau$ \\
\hline$A_{11}$ & 36 & & $-i G^{A} F$ & $-i F F G^{A} F$ & $\tau_{1}<\tau_{2}, \tau_{2}<\tau$ \\
\hline$A_{2}$ & 36 & & $-i G^{R} F$ & $i G^{R} F G^{A} G^{A}$ & $\tau_{2}<\tau_{1}, \tau_{1}<\tau$ \\
\hline$A_{21}$ & 36 & & $-i G^{A} F$ & $i G^{R} G^{R} G^{A} F$ & $\tau_{1}<\tau_{2}, \tau_{2}<\tau$ \\
\hline$B$ & 18 & & $F F$ & $-G^{R} F G^{A} F$ & $\tau_{1}<\tau, \tau_{2}<\tau$ \\
\hline$C_{1}$ & 18 & & $-G^{R} G^{R}$ & $-G^{R} F G^{A} F$ & $\tau_{2}<\tau_{1}, \tau_{1}<\tau$ \\
\hline$C_{2}$ & 18 & & $-G^{A} G^{A}$ & $-G^{R} F G^{A} F$ & $\tau_{1}<\tau_{2}, \tau_{2}<\tau$ \\
\hline
\end{tabular}

As observed in Minkowski spacetime, for all diagrams the loop contribution is singular. Nevertheless the sum $\left(G^{A}\right)^{2}+\left(G^{R}\right)^{2}-F^{2}$ is regular in the ultraviolet regime and will give a finite contribution. The only true UV divergence is given by the singular product $F G^{R}$ and is described by the following loop integral

$$
\int \frac{d^{3} p_{1}}{(2 \pi)^{3}} \int \frac{d^{3} p_{2}}{(2 \pi)^{3}} F\left(p_{1}, t_{1}, t_{2}\right) G^{R}\left(p_{2}, t_{1}, t_{2}\right)(2 \pi)^{3} \delta\left(\mathbf{k}_{1}+\mathbf{k}_{2}-\mathbf{p}_{1}-\mathbf{p}_{2}\right)
$$

This and similar integrals are the same as those that appear in the one-loop corrections to the two-point function for a scalar field theory with a cubic self-interaction and were already computed in [143] for a massless field in de-Sitter spacetime. We will 
use their results in the following when calculating the amputated contributions.

The integral of eq. (5.38) can be simplified by exploiting the spherical symmetry in momentum space. Let's write the general form of all loop diagrams listed in Table 5.3.

$$
\int d^{3} p \int d^{3} p^{\prime} \delta^{3}\left(k+\mathbf{p}+\mathbf{p}^{\prime}\right) f\left(k, p, p^{\prime}\right)
$$

We can use the Dirac delta function in order to simplify the momentum integral over $p^{\prime}$

$$
\int d^{3} p f(k, p,|\mathbf{p}+\mathbf{k}|)
$$

Now, by exploiting the radial symmetry of the function $f$ in the first two arguments we can write the integral in spherical coordinates and integrate over the inclination angle $\varphi_{p}$

$$
2 \pi \int_{0}^{\infty} d p p^{2} \int_{-1}^{1} d \cos \theta f\left(k, p, \sqrt{k^{2}+p^{2}+2 k p \cos \theta}\right) .
$$

The integral can be simplified with the definition of the new variable $p^{\prime}=$ $\sqrt{k^{2}+p^{2}+2 k p \cos \theta}$ to

$$
2 \pi \int_{0}^{\infty} d p p^{2} \int_{-|p-k|}^{p+k} d p^{\prime} \frac{p^{\prime}}{p k} f\left(k, p, p^{\prime}\right)=\frac{2 \pi}{k} \int_{0}^{\infty} d p p \int_{|p-k|}^{p+k} d p^{\prime} p^{\prime} f\left(k, p, p^{\prime}\right) .
$$

The integration in the momentum variables cannot be performed analytically without any simplification of the propagators. In the rest of the section we assume that the external momenta are super-Hubble, i.e. with wavelengths above the Hubble radius and we consider the virtual particles to be massless. In the infrared regime we will use $\nu=3 / 2-\varepsilon$ as an infrared regulator as we did in the renormalization of the two-point function.

\section{Contribution $B+C_{1}+C_{2}$}

Let us consider the finite contribution to the four-point function of diagrams $B, C_{1}$ and $C_{2}$. In this case the product of the distributions $F^{2},\left(G^{R}\right)^{2}$ and $\left(G^{A}\right)^{2}$ diverge linearly, but the sum $\left(G^{A}\right)^{2}+\left(G^{R}\right)^{2}-F^{2}$ is UV finite.

The amputated amplitude of diagram $B$ including the contribution of the two vertices reads

$$
\begin{gathered}
B_{\mathrm{amp}}=\left(-\frac{i \lambda}{3 !}\right)^{2} a^{4}\left(\tau_{1}\right) a^{4}\left(\tau_{2}\right) \int \frac{d^{3} p}{(2 \pi)^{3}} \int d^{3} p^{\prime} \delta^{3}\left(\mathbf{k}-\mathbf{p}-\mathbf{p}^{\prime}\right) F\left(p^{\prime}, \tau_{1}, \tau_{2}\right) F\left(p, \tau_{1}, \tau_{2}\right) \\
=\left(-\frac{i \lambda}{3 !}\right)^{2} a^{4}\left(\tau_{1}\right) a^{4}\left(\tau_{2}\right) \frac{1}{k(2 \pi)^{2}} \int_{0}^{\infty} d p p \int_{p-k}^{p+k} d p^{\prime} p^{\prime} F\left(p^{\prime}, \tau_{1}, \tau_{2}\right) F\left(p, \tau_{1}, \tau_{2}\right),
\end{gathered}
$$


where $\tau_{1}$ and $\tau_{2}$ are the virtual times, $k=k_{1}+k_{2}=k_{3}+k_{4}$, and $F$ is the Hadamard propagator in de-Sitter spacetime. Similarly we have the expression for diagrams $C_{1}$ and $C_{2}$

$$
\begin{aligned}
& C_{1, \mathrm{amp}}=-\left(-\frac{\lambda^{2}}{4 ! 3 !}\right) a^{4}\left(\tau_{1}\right) a^{4}\left(\tau_{2}\right) \frac{1}{k(2 \pi)^{2}} \int_{0}^{\infty} d p p \int_{p-k}^{p+k} d p^{\prime} p^{\prime} G^{R}\left(p^{\prime}, \tau_{1}, \tau_{2}\right) G^{R}\left(p, \tau_{1}, \tau_{2}\right), \\
& C_{2, \mathrm{amp}}=-\left(-\frac{\lambda^{2}}{4 ! 3 !}\right) a^{4}\left(\tau_{1}\right) a^{4}\left(\tau_{2}\right) \frac{1}{k(2 \pi)^{2}} \int_{0}^{\infty} d p p \int_{p-k}^{p+k} d p^{\prime} p^{\prime} G^{A}\left(p^{\prime}, \tau_{1}, \tau_{2}\right) G^{A}\left(p, \tau_{1}, \tau_{2}\right) .
\end{aligned}
$$

The full expression corresponding to the different diagrams is given by the renormalized amputated amplitude with the inclusion of the external propagators. Since in the fish diagram there are two vertices, we have to integrate over time twice. The full amplitude reads ${ }^{3}$

$$
\begin{gathered}
{[B]_{\mathrm{ren}}=-18 \int_{\tau_{\mathrm{in}}}^{\tau} d \tau_{1} \int_{\tau_{\mathrm{in}}}^{\tau} d \tau_{2} G^{R}\left(k_{1}, \tau, \tau_{1}\right) F\left(k_{2}, \tau, \tau_{1}\right) G^{A}\left(k_{3}, \tau_{1}, \tau\right) F\left(k_{4}, \tau_{1}, \tau\right)\left[B_{\mathrm{amp}}\right]_{\mathrm{ren}},} \\
{\left[C_{i}\right]_{\mathrm{ren}}=-18 \int_{\tau_{\mathrm{in}}}^{\tau} d \tau_{1} \int_{\tau_{\mathrm{in}}}^{\tau} d \tau_{2} G^{R}\left(k_{1}, \tau, \tau_{1}\right) F\left(k_{2}, \tau, \tau_{1}\right) G^{A}\left(k_{3}, \tau_{1}, \tau\right) F\left(k_{4}, \tau_{1}, \tau\right)\left[C_{i, \mathrm{amp}}\right]_{\mathrm{ren}} .}
\end{gathered}
$$

To perform the momentum integration of the singular product of the Hadamard propagator $F^{2}$ of diagram B, one can split the integral in two parts: the small momentum contribution where the Hankel propagators are taken as in eqs. (4.29) and (4.30) with $\nu=3 / 2-\varepsilon$ and the large momentum contribution. The small momentum contribution reads

$$
B^{s}=\frac{H^{4}\left(2 \log \left(k^{2} \tau_{1} \tau_{2}\right)+\frac{k}{M}-\frac{1}{2} \log \left(\frac{k+M}{M-k}\right)+\frac{1}{\varepsilon}\right)}{8 \pi^{2} k^{3}},
$$

where $k=k_{1}+k_{2}$ and $M$ is the mass scale used as the upper limit for the momentum integral. The large momentum is

$$
B^{l}=\frac{H^{4}\left(\frac{1}{2} \log \left(\frac{k+M}{M-k}\right)-\frac{k}{M}\right)}{8 \pi^{2} k^{3}}-\frac{H^{3} \Lambda \tau_{1}^{2} \tau_{2}^{2} \sin \left(k\left(\tau_{1}-\tau_{2}\right)\right)}{16 \pi^{2} k \tau_{x}\left(\tau_{1}-\tau_{2}\right)} .
$$

Here $\tau_{x}$ comes from the physical cutoff $-\Lambda /\left(H \tau_{x}\right)$, where $\tau_{x}=\min \left(\tau_{1}, \tau_{2}\right)$. In other words we regulate our theory with the largest cutoff $\max \left(\Lambda a\left(\tau_{1}\right), \Lambda a\left(\tau_{2}\right)\right)$. The linear dependence of this expression will be compensated with the contribution from diagrams $C_{1}$ and $C_{2}$.

\footnotetext{
${ }^{3}$ The small and large momenta contributions $B^{s}, B^{l}$ and $C^{l}$ are taken from [143].
} 
Diagram $\mathrm{C}$ is regular in the infrared regime and does not give late time contributions. The large momenta contributions for diagrams $C_{1}$ and $C_{2}$ is calculated in a similar way and read

$$
\begin{aligned}
C_{1}^{l} & =\frac{H^{3} \Lambda \tau_{1}^{2} \tau_{2}^{2}}{4 k \pi^{2}\left(\tau_{1}-\tau_{2}\right) \tau_{x}} \sin \left(k\left(\tau_{1}-\tau_{2}\right)\right) \theta\left(\tau_{1}-\tau_{2}\right), \\
C_{2}^{l} & =\frac{H^{3} \Lambda \tau_{1}^{2} \tau_{2}^{2}}{4 k \pi^{2}\left(\tau_{2}-\tau_{2}\right) \tau_{x}} \sin \left(k\left(\tau_{2}-\tau_{1}\right)\right) \theta\left(\tau_{2}-\tau_{1}\right)
\end{aligned}
$$

The two contributions can be easily summed-up. We observe that the sum does not depend on any step-function because the factor $\theta\left(\tau_{1}-\tau_{2}\right)$ is compensated by the symmetric contribution $\theta\left(\tau_{2}-\tau_{1}\right)$. We have

$$
C^{l}=C_{1}^{l}+C_{2}^{l}=\frac{H^{3} \Lambda \tau_{1}^{2} \tau_{2}^{2}}{4 k \pi^{2}\left(\tau_{1}-\tau_{2}\right) \tau_{x}} \sin \left(k\left(\tau_{1}-\tau_{2}\right)\right)
$$

The amputated contribution $\left[B+C_{1}+C_{2}\right]_{\mathrm{amp}}$ is finally given by the sum of all small and large momenta contributions with the inclusion of two vertices $\lambda^{2} a^{4}\left(\tau_{1}\right) a^{4}\left(\tau_{2}\right)$ with the corresponding combinatorial factor.

$$
\left[B+C_{1}+C_{2}\right]_{\mathrm{amp}}=\left(-\frac{i \lambda}{3 !}\right)^{2} a^{4}\left(\tau_{1}\right) a^{4}\left(\tau_{2}\right)\left(B^{s}+B^{l}\right)+\left(-\frac{i \lambda}{4 !}\right)\left(-\frac{i \lambda}{3 !}\right) a^{4}\left(\tau_{1}\right) a^{4}\left(\tau_{2}\right) C^{l}
$$

The explicit expression reads

$$
-\frac{\lambda^{2}\left(2 \log \left(\left(k_{1}+k_{2}\right)^{2} \tau_{1} \tau_{2}\right)+\frac{1}{\varepsilon}\right)}{288 \pi^{2} H^{4}\left(k_{1}+k_{2}\right)^{3} \tau_{1}^{4} \tau_{2}^{4}}
$$

and is infrared divergent and finite in the ultraviolet regime (as expected from previous considerations). The last part consists in the evaluation of the full contribution to the correlation function considering also the external propagators as in eq. (5.46). In order to perform the time integrals analytically we assumed the propagators in the limit of small $|k \tau|$ and they are given in eq. (4.35).

Using the integrals (B.1) and (B.2) in Appendix B, we obtain the full expression for the contribution $B+C_{1}+C_{2}$ that reads

$$
\begin{aligned}
& \frac{H^{4} \lambda^{2}\left(k_{1}^{3}+k_{2}^{3}\right)\left(k_{3}^{3}+k_{4}^{3}\right)\left(3 \log \left(\frac{\tau}{\tau_{\text {in }}}\right)+1\right)}{15552 \pi^{2}\left(k_{1}+k_{2}\right)^{3} k_{1}^{3} k_{2}^{3} k_{3}^{3} k_{4}^{3}}\left(9 \log \left(\frac{\tau}{\tau_{\text {in }}}\right)\left(2 \log \left(k^{2} \tau \tau_{\text {in }}\right)+\frac{1}{\varepsilon}\right)\right. \\
& \left.+12 \log (-k \tau)+4+\frac{3}{\varepsilon}\right) .
\end{aligned}
$$


As for the two-point function the correction has a logarithmic dependence on the conformal time, in this case quadratic, and the first-order contributions of the oscillatory terms. It depends also on the infrared cutoff $\varepsilon$ as expected.

\section{Contribution $A_{1}+A_{11}$}

Similarly we discuss the contribution of diagrams $A_{1}$ and $A_{11}$, that involve the singular product of the propagators $F$ and $G^{A / R}$. In this case we deal with a true divergence that is not compensated by the other diagrams and we need to renormalize it with the universal coupling constant counter-term that we found in Minkowski spacetime

$$
\delta \lambda=-3 \frac{\lambda^{2}}{16 \pi^{2}} \log \frac{\Lambda}{\mu}
$$

which fully agrees with similar results in the literature (see [84] and the computations in different schemes in [33] and [34]).

The amputated amplitudes for diagrams $A_{1}$ and $A_{11}$ including the contribution from the vertices are

$$
\begin{aligned}
& A_{1, \mathrm{amp}}=-i\left(-\frac{i \lambda}{3 !}\right)^{2} a^{4}\left(\tau_{1}\right) a^{4}\left(\tau_{2}\right) \frac{1}{k(2 \pi)^{2}} \int_{0}^{\infty} d p p \int_{p-k}^{p+k} d p^{\prime} p^{\prime} G^{R}\left(p^{\prime}, \tau_{1}, \tau_{2}\right) F\left(p, \tau_{1}, \tau_{2}\right), \\
& A_{11, \mathrm{amp}}=-i\left(-\frac{i \lambda}{3 !}\right)^{2} a^{4}\left(\tau_{1}\right) a^{4}\left(\tau_{2}\right) \frac{1}{k(2 \pi)^{2}} \int_{0}^{\infty} d p p \int_{p-k}^{p+k} d p^{\prime} p^{\prime} G^{A}\left(p^{\prime}, \tau_{1}, \tau_{2}\right) F\left(p, \tau_{1}, \tau_{2}\right),
\end{aligned}
$$

where we used the same notation of the previous example. The full amplitudes with the inclusion of the external propagators read

$$
\begin{gathered}
{\left[A_{1}\right]_{\mathrm{ren}}=-36 i \int_{\tau_{\mathrm{in}}}^{\tau} d \tau_{1} \int_{\tau_{\mathrm{in}}}^{\tau} d \tau_{2} G^{R}\left(k_{1}, \tau, \tau_{1}\right) F\left(k_{2}, \tau, \tau_{1}\right) F\left(k_{3}, \tau_{1}, \tau\right) F\left(k_{4}, \tau_{1}, \tau\right)\left[A_{1, \mathrm{amp}}\right]_{\mathrm{ren}},} \\
{\left[A_{11}\right]_{\mathrm{ren}}=-36 i \int_{\tau_{\mathrm{in}}}^{\tau} d \tau_{1} \int_{\tau_{\mathrm{in}}}^{\tau} d \tau_{2} F\left(k_{1}, \tau, \tau_{1}\right) F\left(k_{2}, \tau, \tau_{1}\right) G^{A}\left(k_{3}, \tau_{1}, \tau\right) F\left(k_{4}, \tau_{1}, \tau\right)\left[A_{11, \mathrm{amp}}\right]_{\mathrm{ren}} .}
\end{gathered}
$$

Similarly to what it has been done before, we investigate the contribution of diagrams $A_{i}$ s, that involve the singular product of the propagators $F$ and $G^{A / R}$. The UV behavior was studied in [143] for a $\lambda \phi^{3}$-theory. They found an analytic expression for the renormalized product $F G^{R}$, that in our setup corresponds to 


$$
\begin{aligned}
& -\frac{i H^{4}}{24 \pi^{2}} \theta\left(\tau_{1}-\tau_{2}\right)\left[2 \tau_{1}^{3} \log \left(\frac{\left|\frac{\tau_{2}}{\tau_{1}-\tau_{2}}\right|}{2}\right)\right. \\
& \quad+\frac{3}{2} \tau_{1}^{2} \tau_{2}^{2}\left(\delta\left(\tau_{1}-\tau_{2}\right)\left(\log \left(-\frac{2 \eta \mu}{H \tau_{1}}\right)+\gamma\right)+\frac{\theta\left(-\eta+\tau_{1}-\tau_{2}\right)}{\tau_{1}-\tau_{2}}\right) \\
& \left.-2 \tau_{2}^{3} \log \left(\frac{\tau_{1}}{2\left(\tau_{1}-\tau_{2}\right)}\right)-2 \tau_{1} \tau_{2}\left(\tau_{1}-\tau_{2}\right)+\left(\frac{1}{\epsilon}+\frac{14}{3}-2 \gamma\right)\left(\tau_{1}^{3}-\tau_{2}^{3}\right)\right],
\end{aligned}
$$

where again $\varepsilon$ is the mass regulator introduced to cure the infrared divergence and $\mu$ is the arbitrary renormalization energy scale. The ultraviolet divergence is regulated with the coupling constant counter-term found in Minkowski spacetime (5.54). We remark that the previous expression is consistent with the calculation of [37] performed with the analytic regularization scheme in position space, with the peculiarity that it is compatible with the Epstein-Glaser renormalization in the algebraic quantum field theory approach. They found a renormalized amplitude in Fourier space given by

$$
\begin{aligned}
& \left(\Delta_{F}^{2}\right)_{\text {ren }}=-\frac{1+2 \log (a)}{16 \pi^{2} a^{4}\left(\tau_{1}\right)} i \delta\left(\tau_{1}-\tau_{2}\right)+ \\
& \frac{1}{2 \pi\left(8 \pi^{2}\right) a^{2}\left(\tau_{1}\right) a^{2}\left(\tau_{2}\right)}\left(\partial_{\tau_{1}}^{2}+k^{2}\right) \int_{\mathbb{R}^{3}} d^{3} p\left(\frac{1}{2}\left(\frac{1}{p^{3}}\right)_{\text {ren }}+\frac{i\left|\tau_{1}-\tau_{2}\right|}{2 p^{2}}\right) e^{-i p\left|\tau_{1}-\tau_{2}\right|} \frac{e^{-i|k-p|\left|\tau_{1}-\tau_{2}\right|}}{2|k-p|}
\end{aligned}
$$

where $\Delta_{F}$ is the Feynman propagator.

The first line is compatible with eq. (5.58), where we both observe a dependence on $\log a(\tau)$. This term is originated from the renormalization of the logarithmic divergence in the four-point function. We remark that the overall factor $1 /\left(16 \pi^{2} a^{4}\left(\tau_{1}\right)\right)$ of eq. (5.58) is consistent with eq. (5.59). The only difference is the constant $\gamma$ in eq. (5.58) which is not present in the second expression because they used a different prescription. The second line is instead expressed in terms of a momentum integral where the finite part depends on the renormalization scheme and therefore cannot be directly compared to eq. (5.58).

By assuming the external propagators in the limit of $|k \tau| \ll 1$ and by using the integrals (B.3) - (B.6) in Appendix B, we obtain the full expression for the contribution $A_{1}+A_{11}$ that reads 


$$
\begin{aligned}
& -\frac{H^{4} \lambda^{2}\left(k_{1}^{3}+k_{2}^{3}+k_{3}^{3}+k_{4}^{3}\right)}{62208 \pi^{2} k_{1}^{3} k_{2}^{3} k_{3}^{3} k_{4}^{3}}\left[51 \pi^{2}+126 \gamma-776+126 \log (2)-\frac{36}{\varepsilon}\right. \\
& +54\left(\log \left(\frac{\mu}{H}\right)+4 \zeta(3)\right)+18 \log ^{2}\left(\frac{\tau}{\tau_{\text {in }}}\right)\left(2 \log \left(\frac{8 \tau_{\text {in }}}{\tau}\right)+2(3 \gamma-8)-\frac{3}{\varepsilon}\right) \\
& \left.\quad+6 \log \left(\frac{\tau}{\tau_{\text {in }}}\right)\left(27 \log \left(\frac{\mu}{H}\right)+6 \pi^{2}+51(\gamma+\log (2))-127-\frac{12}{\varepsilon}\right)\right] .
\end{aligned}
$$

We observe that the correction to the correlation function has a quadratic logarithmic dependence on the conformal time that was present also in diagrams $B$ and $C_{i}$ s. The first orders of the early oscillations are also present. Finally the result is consistently regulated with the infrared cutoff $\varepsilon$.

\section{Contribution $A_{2}+A_{21}$}

To conclude, we compute the contribution of the remaining diagrams $A_{2}$ and $A_{22}$ that are renormalized with the coupling constant counter-term (5.54). The loop contribution is given by the singular product $F G^{R}$ that was calculated before. The amputated amplitudes with the inclusion of the contributions of the vertices read

$$
\begin{aligned}
& A_{2, \mathrm{amp}}=-i\left(-\frac{\lambda^{2}}{4 ! 3 !}\right) a^{4}\left(\tau_{1}\right) a^{4}\left(\tau_{2}\right) \frac{1}{k(2 \pi)^{2}} \int_{0}^{\infty} d p p \int_{p-k}^{p+k} d p^{\prime} p^{\prime} G^{R}\left(p^{\prime}, \tau_{1}, \tau_{2}\right) F\left(p, \tau_{1}, \tau_{2}\right), \\
& A_{21, \mathrm{amp}}=-i\left(-\frac{\lambda^{2}}{4 ! 3 !}\right) a^{4}\left(\tau_{1}\right) a^{4}\left(\tau_{2}\right) \frac{1}{k(2 \pi)^{2}} \int_{0}^{\infty} d p p \int_{p-k}^{p+k} d p^{\prime} p^{\prime} G^{A}\left(p^{\prime}, \tau_{1}, \tau_{2}\right) F\left(p, \tau_{1}, \tau_{2}\right) .
\end{aligned}
$$

The full amplitudes are given by

$$
\begin{gathered}
{\left[A_{2}\right]_{\mathrm{ren}}=36 i \int_{\tau_{\text {in }}}^{\tau} d \tau_{1} \int_{\tau_{\text {in }}}^{\tau} d \tau_{2} G^{R}\left(k_{1}, \tau, \tau_{1}\right) F\left(k_{2}, \tau, \tau_{1}\right) G^{A}\left(k_{3}, \tau_{1}, \tau\right) G^{A}\left(k_{4}, \tau_{1}, \tau\right)\left[A_{2, \mathrm{amp}}\right]_{\mathrm{ren}},} \\
{\left[A_{21}\right]_{\mathrm{ren}}=36 i \int_{\tau_{\mathrm{in}}}^{\tau} d \tau_{1} \int_{\tau_{\mathrm{in}}}^{\tau} d \tau_{2} G^{R}\left(k_{1}, \tau, \tau_{1}\right) G^{R}\left(k_{2}, \tau, \tau_{1}\right) G^{A}\left(k_{3}, \tau_{1}, \tau\right) F\left(k_{4}, \tau_{1}, \tau\right)\left[A_{21, \mathrm{amp}}\right]_{\mathrm{ren}} .}
\end{gathered}
$$

Now the full amplitude differs from that computed for diagrams $A_{1}$ and $A_{11}$ because of the different external propagators (and vertices). Therefore, with the integrals (B.7) 
- (B.10) given in Appendix B we obtain the full contribution $A_{2}+A_{21}$

$$
\begin{aligned}
& -\frac{H^{4} \lambda^{2} \tau_{\text {in }}^{6}\left(k_{1}^{3} k_{2}^{3} k_{3}^{3}+k_{1}^{3} k_{2}^{3} k_{4}^{3}+k_{1}^{3} k_{3}^{3} k_{4}^{3}+k_{2}^{3} k_{3}^{3} k_{4}^{3}\right)}{186624 \pi^{2} k_{1}^{3} k_{2}^{3} k_{3}^{3} k_{4}^{3}}\left(9 \log \left(\frac{\mu}{H}\right)+\frac{1}{\varepsilon} \log \left(\frac{\tau_{\text {in }}^{6}}{\tau^{6}}\right)+\right. \\
& \left.2\left(3 \log \left(\frac{4 \tau_{\text {in }}}{\tau}\right)+6 \gamma-17\right) \log \left(\frac{\tau}{\tau_{\text {in }}}\right)+2 \pi^{2}+19 \gamma-48+19 \log (2)-\frac{5}{\varepsilon}\right) .
\end{aligned}
$$

Again we observe the expected logarithmic dependence on the conformal time and the first-order contributions of the periodic terms. We remark that the corrections are proportional and suppressed by the overall factor $\lambda^{2}$. Therefore the loop contributions are negligible compared to the tree-level result.

The tree-level contribution and the sum of all one-loop corrections are shown in Figure 5.9, where the radiative corrections have been enhanced by a factor of $10^{13}$. It should be noted that the loop corrections do not have any periodic features since we considered the external propagators to be in the super-Hubble regime. We expect that the oscillations are present in the general result obtained with the full propagators.

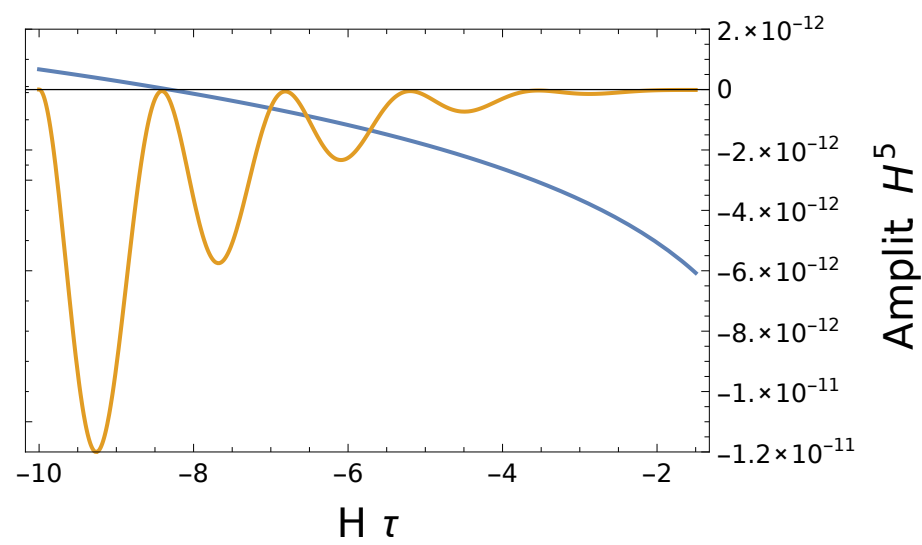

Figure 5.9: Tree-level (yellow line) and one-loop contributions (blue line) to the fourpoint function where we set $k_{1,2,3,4} / H=1, \lambda=10^{-13}$ and $H \tau_{\text {in }}=-10$. The loop contribution is amplified by a factor $10^{13}$.

We are at the end of our discussion about the equal-time four-point function renormalization in Minkowski and de-Sitter spacetime using the closed-time-path formalism. In Chapter 7 we will discuss the physical consequences of our results and we will give our predictions to the non-linearity parameter $\tau_{\mathrm{NL}}$ that describes the non-Gaussian properties of the inflationary physics in the primordial universe. 


\subsection{Massive WKB counter-term in de-Sitter space- time}

The full propagator for a massive theory in de-Sitter spacetime is given by the hypergeometric function (4.39), which is an implicit function of the coordinates. It is therefore difficult to disentangle the divergences from the finite contributions in the perturbative expansion of the correlation functions.

The WKB propagator (4.46) gives a very good approximation of the full massive propagator in the UV regime and for very large masses $m \gg H$. In the following we study the massive counter-term for a two scalar field theory with Feynman rules given in the Appendix A.2 where the light field gets radiative corrections from the heavy field described by the WKB propagator.

After having introduced a physical cutoff $\Lambda$ that regulates the momentum integral of the WKB propagator (4.47), the one-loop correction to the two-point function 5.13 reads

$$
\left[A_{\mathrm{amp}}\right]_{\mathrm{reg}}=-i \lambda_{h}^{2} \frac{\Lambda \sqrt{\Lambda^{2}+m^{2}}-m^{2} \sinh ^{-1}\left(\frac{\Lambda}{m}\right)}{8 \pi^{2}} .
$$

In order to renormalize this amplitude, we extracted the UV divergence and defined the mass counter-term

$$
\delta m^{2}=\frac{\lambda_{h}^{2}}{4 \pi^{2}}\left(\Lambda^{2}-m^{2} \log \left(\frac{\Lambda}{\mu}\right)\right)
$$

which is consistent with the analogous counter-term (5.10) found in Minkowski spacetime ${ }^{4}$. The second term in eq. (5.66) is not present in the massless case, because it is proportional to the squared mass.

After the subtraction of the ultraviolet divergences we obtain the analytic expression for the renormalized one-loop correction in the WKB approximation

$$
\left[A_{\mathrm{amp}}\right]_{\mathrm{ren}}=\frac{-i \lambda_{h}^{2} m^{2} a^{4}(\tau)}{4 \pi^{2}}\left(1+\log \left(\frac{m^{2}}{4 \mu^{2}}\right)\right) \text {. }
$$

Contrary to the massless case, where the one-loop correction was proportional to the Hubble parameter $H^{2}(\tau)$, now it is proportional to $m^{2}$, which is larger than $H^{2}(\tau)$ for non dynamical fields during inflation.

It is therefore not sufficient to renormalize the tadpole derived numerically with the full massive propagator by subtracting only the UV divergences found in the massless case, because there will be the residual logarithmic divergence that depends on the

\footnotetext{
${ }^{4}$ It should be noted that here we are using the Feynam rules for the hybrid model, explaining the different normalization.
} 
mass. Our strategy is to subtract instead the counter-term computed analytically with the WKB propagator, which is consistent to the analytic expression found in Minkowski spacetime in eq. (5.10), and to include the contribution of the massless counter-term (5.20), that does not appear in the WKB approximation.

In Figure 5.10 we show the squared mass dependence of the residual finite part for the amputated diagram with the full massive propagator in the minimal subtraction scheme which consistently matches the one predicted in eq. (5.67).

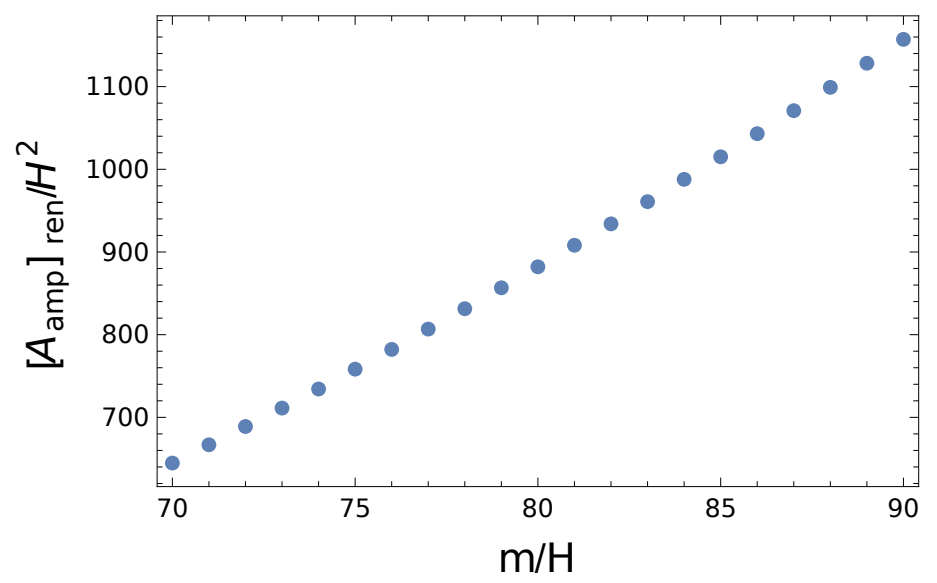

Figure 5.10: Mass dependence of the amputated tadpole diagram with the full hypergeometric propagator after renormalization with the WKB couter-term.

The last part of this section is devoted to the precision of the numerical methods that we used in order to approximate the Schwinger propagator obtained from the hypergeometric function. The hypergeometric propagator is evaluated in momentum space for a finite set of points as shown in Figure 4.3. The discrete points are subsequently fitted with a polynomial function in order to be able to proceed with the renormalization procedure analytically. In Figure 5.11 we show the relative difference between the hypergeometric and the WKB propagator for a very massive field.

We observe very small oscillations in the difference and an apparent increase at large $k$. This can be consistently explained considering that we are fitting the Fourier transformed hypergeometric function on a discrete set of points with a polynomial function exactly on this range. We expect that at the boundaries the fit becomes less reliable. So the last part of that range will be excluded in the analysis. Additionally we verified for the masses of interest that the integrated difference, which is the important quantity for the loop integral, is always well-behaved and less than $0.001 \%$. 


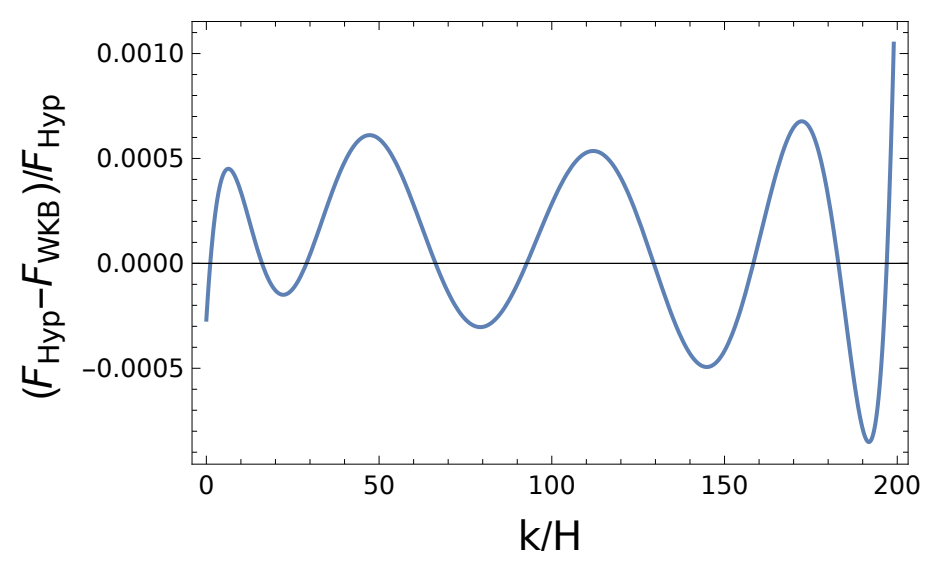

Figure 5.11: Relative difference between the Hypergeometric and the WKB propagator for a scalar field of mass $60 \mathrm{H}$.

\subsection{Comparison of the counter-terms obtained in the adiabatic regularization scheme}

In the previous sections the two- and four-point correlation functions were renormalized in the language of the Schwinger and Keldysh formalism for a massless and massive theory in Minkowski and de-Sitter spacetime. In order to obtain finite quantities we used the minimal subtraction scheme where we defined the mass, curvature and coupling constant counter-terms (5.10), (5.20), (5.66), and (5.54). It would be interesting to compare our results with counter-terms obtained using a completely different and independent renormalization scheme.

In the following section, the methods of adiabatic renormalization introduced in Section 3 will be applied to give an independent calculation of counter-terms. Here we will renormalize the adiabatic energy-momentum tensor (3.30) on a n-dimensional FRW spacetime. The methods that we use are explained in [34] and combine the benefits of adiabatic subtraction and the effective action approach.

The one-loop corrections of eq. (3.30) were computed using the methods of the effective action where the integral is regular because we are working on a n-dimensional spacetime. Counter-terms $\delta T_{\mu \nu}$ are fixed by requiring that the effective theory matches the classical one. In the case of the energy-momentum tensor (3.30) the one-loop corrections are regularized by subtracting the 4 th order adiabatic expansion ${ }^{5}$. This procedure will give an explicit expression for all counter-terms defined in eq. (3.31) and they can be found by comparing the regularized energy-momentum tensor (3.30) with the classical one [34]

\footnotetext{
${ }^{5}$ It is necessary to consider the subtraction at least at the 4 th order to cancel completely all the divergences that appear in the regulated expression. Higher orders are not relevant and will have an impact only on the finite part.
} 


$$
T_{00}^{C}=\frac{1}{2}\left[\dot{\varphi}^{2}+m^{2} \varphi^{2}+2 \frac{\lambda}{4 !} \varphi^{4}\right]+\xi\left[(n-1)\left(\frac{n}{2}-1\right)\left(\frac{\dot{a}}{a}\right)^{2} \varphi^{2}+2(n-1) \frac{\dot{a}}{a} \dot{\varphi} \varphi\right]
$$

In the following this procedure is tested by assuming the renormalization conditions in Minkowski spacetime and in de-Sitter spacetime. In the first case we expect to recover the same results as in [34]. In the second case we expect a difference only in the finite part because of the universality of the divergences in curved spacetime.

It should be noted that by comparing the adiabatic counter-terms with those found in our previous calculations the finite part will differ because here we are not working in the same prescription and the adiabatic finite part strongly depends on the order in the adiabatic expansion. We point-out that since the procedure is fully covariant, including the counter-terms defined in eq. (3.31), we expect that the results will preserve this covariance.

\section{Renormalization conditions on a Minkowski background}

In the following section we express the counter-terms by comparing the regulated energy-momentum tensor in eq. (3.31) with the classical expression in eq. (5.68) by imposing the renormalization conditions on a flat spacetime $a(t)=1$. These conditions can be read directly from eq. (5.68)

$$
\begin{aligned}
\left.\frac{\partial^{2}\left\langle T_{00}\right\rangle}{\partial \dot{\varphi}^{2}}\right|_{a=1}=1, & \left.\frac{\partial^{2}\left\langle T_{00}\right\rangle}{\partial \varphi^{2}}\right|_{a=1}=m^{2}, & \left.\frac{\partial^{4}\left\langle T_{00}\right\rangle}{\partial \varphi^{4}}\right|_{a=1}=\lambda \\
\left.\frac{\partial^{3}\left\langle T_{00}\right\rangle}{\partial \varphi \partial \dot{\varphi} \partial(\dot{a} / a)}\right|_{a=1}=2 \xi(n-1), & \left.\left\langle T_{00}\right\rangle\right|_{a=1}=0, & \left.\frac{\partial^{2}\left\langle T_{00}\right\rangle}{\partial(\dot{a} / a)^{2}}\right|_{a=1}=0, \\
\left.\frac{\partial^{3}\left\langle T_{00}\right\rangle}{\partial(\dot{a} / a)^{2} \partial(\ddot{a} / a)}\right|_{a=1}=0, & \left.\frac{\partial^{2}\left\langle T_{00}\right\rangle}{\partial(\ddot{a} / a)^{2}}\right|_{a=1}=0, & \left.\frac{\partial^{4}\left\langle T_{00}\right\rangle}{\partial(\dot{a} / a)^{4}}\right|_{a=1}=0 .
\end{aligned}
$$

With these renormalization conditions with a fourth order adiabatic expansion in the subtraction scheme we found the following counter-terms 


$$
\begin{aligned}
\delta m^{2} & =\frac{-m^{2} \lambda}{16(n-4) \pi^{2}}+\frac{m^{2} \lambda}{32 \pi^{2}}\left(1-\gamma+\log \left(\frac{4 \pi \mu^{2}}{m^{2}}\right)\right), \\
\delta \lambda & =\frac{-3 \lambda^{2}}{16(n-4) \pi^{2}}+\frac{3 \lambda^{2}}{32 \pi^{2}}\left(-\gamma+\log \left(\frac{4 \pi \mu^{2}}{m^{2}}\right)\right), \\
\delta \Lambda & =\frac{m^{4}}{32(n-4) \pi^{2}}+\frac{m^{4}}{128 \pi^{2}}\left(-3+2 \gamma-2 \log \left(\frac{4 \pi \mu^{2}}{m^{2}}\right)\right), \\
\delta \xi & =\frac{(1-6 \xi) \lambda}{96(n-4) \pi^{2}}+\frac{\lambda(-1+6 \xi)}{192 \pi^{2}}\left(-\gamma+\log \left(\frac{4 \pi \mu^{2}}{m^{2}}\right)\right), \\
\delta \alpha & =\frac{m^{2}(-1+6 \xi) \lambda}{96(n-4) \pi^{2}}+\frac{m^{2}(-1+6 \xi)}{192 \pi^{2}}\left(-1+\gamma-\log \left(\frac{4 \pi \mu^{2}}{m^{2}}\right)\right),
\end{aligned}
$$

where $n$ denotes the dimensionality of the space, $\mu$ is the renormalization scale and the pole in $1 /(n-4)$ is the usual pole that one finds in dimensional regularization. As expected the results are covariant expression and no time dependence appears because we set the initial condition to be on a flat spacetime. These results are of course consistent with those in [34] because we applied the same method. The normalization is also consistent with our previous results and with analogous results in the literature [33] where they computed the same counter-terms using a similar prescription.

\section{Renormalization conditions on a de-Sitter background}

The same procedure is applied using the same renormalization conditions for a different background. In the following example we will extend the results of [34] by choosing the initial conditions given on a curved background described by the de-Sitter metric, where the scale factor is expressed in coordinate time $a(t)=e^{H t}$. The renormalization conditions read

$$
\begin{aligned}
& \left.\frac{\partial^{2}\left\langle T_{00}\right\rangle}{\partial \dot{\varphi}^{2}}\right|_{a=e^{H t}}=1,\left.\quad \frac{\partial^{2}\left\langle T_{00}\right\rangle}{\partial \varphi^{2}}\right|_{a=e^{H t}}=m^{2},\left.\quad \frac{\partial^{4}\left\langle T_{00}\right\rangle}{\partial \varphi^{4}}\right|_{a=e^{H t}}=\lambda, \\
& \left.\frac{\partial^{3}\left\langle T_{00}\right\rangle}{\partial \varphi \partial \dot{\varphi} \partial(\dot{a} / a)}\right|_{a=e^{H t}}=2 \xi(n-1),\left.\quad\left\langle T_{00}\right\rangle\right|_{a=e^{H t}}=0,\left.\quad \frac{\partial^{2}\left\langle T_{00}\right\rangle}{\partial(\dot{a} / a)^{2}}\right|_{a=e^{H t}}=0, \\
& \left.\frac{\partial^{3}\left\langle T_{00}\right\rangle}{\partial(\dot{a} / a)^{2} \partial(\ddot{a} / a)}\right|_{a=e^{H t}}=0,\left.\quad \frac{\partial^{2}\left\langle T_{00}\right\rangle}{\partial(\ddot{a} / a)^{2}}\right|_{a=e^{H t}}=0,\left.\quad \frac{\partial^{4}\left\langle T_{00}\right\rangle}{\partial(\dot{a} / a)^{4}}\right|_{a=e^{H t}}=0 \text {. }
\end{aligned}
$$


The time dependence of the scale factor complicates the calculation of the counterterms. It is still possible to find the full analytic solution .

$$
\begin{aligned}
& \delta m^{2}= \frac{-m^{2} \lambda}{16(n-4) \pi^{2}}+\frac{m^{2} \lambda}{32 \pi^{2}}\left(1-\gamma+\log \left(\frac{4 \pi \mu^{2}}{m^{2}}\right)\right)-6 H^{2} \xi+ \\
& \quad+\frac{5 H^{2} \lambda(1-6 \xi)}{96 \pi^{2}}+\frac{H^{4} \lambda}{160 m^{2} \pi^{2}}(33+10 \xi(-31+72 \xi)) \\
& \delta \lambda= \frac{-3 \lambda^{2}}{16(n-4) \pi^{2}}+\frac{3 \lambda^{2}}{32 \pi^{2}}\left(-\gamma+\log \left(\frac{4 \pi \mu^{2}}{m^{2}}\right)\right)+\frac{3 H^{2} \lambda^{2}}{32 \pi^{2} m^{2}}(1-6 \xi) \\
& \delta \Lambda= \frac{m^{4}}{32(n-4) \pi^{2}}+\frac{m^{4}}{128 \pi^{2}}\left(-3+2 \gamma-2 \log \left(\frac{4 \pi \mu^{2}}{m^{2}}\right)\right)+\frac{5 H^{2} m^{2}}{96 \pi^{2}}(-1+6 \xi)+ \\
& \quad+\frac{H^{4}}{2880 \pi^{2}}(209+2520 \xi(-1+3 \xi)) \\
& \delta \xi= \frac{(1-6 \xi) \lambda}{96(n-4) \pi^{2}}+\frac{\lambda(-1+6 \xi)}{192 \pi^{2}}\left(-\gamma+\log \left(\frac{4 \pi \mu^{2}}{m^{2}}\right)\right)+ \\
& \quad+\frac{H^{2} \lambda}{960 m^{2} \pi^{2}}(-33+10(31-72 \xi) \xi) \\
& \quad+\frac{H^{2}}{8540 \pi^{2}}(-61+720(1-3 \xi) \xi)
\end{aligned}
$$

We found that the counter-terms are given by the dimensional regularization poles in $1 /(n-4)$ that are universal and do not depend on the background we consider. The finite part that we found in the case of a Minkowski spacetime is also contributing. The only difference is in those terms that are written in blue color that depend on the squared Hubble parameter $H^{2}$. Because in a FRW spacetime the curvature is given by $\mathcal{R}(t)=12 H^{2}(t)$, the finite part can be expressed in terms of geometrical quantities and the contribution proportional to $H^{2}$ can be interpreted as a covariant correction to the curvature term. Moreover by sending the curvature $\mathcal{R}$ to zero, we consistently recover the results obtained on a flat spacetime.

In this section we analyzed the one-loop correction to the energy-momentum tensor in the effective action approach using adiabatic regularization in order to obtain an independent derivation of counter-terms. The results are fully consistent with those obtained in the theoretical framework of the Schwinger and Keldysh formalism. The only difference is in the finite part that nevertheless could be expressed in terms of geometrical covariant quantities as the metric and the curvature. We point-out that in the counter-terms defined in the CTP formalism the finite part is zero because we are subtracting the divergences in the minimal subtraction scheme. 


\section{CHAPTER 6 LINTERACTION PROFILE AND THE ADIABATIC LIMIT}

In the previous chapter the Schwinger and Keldysh formalism was applied in order to calculate the explicit time-evolution of the renormalized two- and four-point correlation function for different backgrounds. In the case of a flat spacetime, because the background is not breaking the time translational symmetry of the system, no explicit time dependence from the metric was found. On the other hand, because the closed-time-path formalism sets an initial time $t_{\text {in }}$ where the interaction is switched-on, we found in both cases oscillatory features arising from the contribution of the external propagators that depend on the initial time. In quasi de-Sitter spacetime we found periodic contributions arising from the external propagators and a logarithmic time dependence $\log H$ coming from the time-evolution of the universe encoded in the background.

In the considered examples of renormalization in Minkowski spacetime we expect to recover the Poincaré invariance in the limit of large elapsed time $t-t_{\text {in }} \gg 1$. This can be seen by interpreting the in-in formalism in the equivalent scenario of a scalar field theory that freely evolves from infinite negative time to the initial time according to the free theory. At this time the interaction is switched-on and the system evolves according to the full theory and we stop to be on an eigenstate of the system. This picture can be analytically represented through a step-function $\theta\left(t-t_{\text {in }}\right)$ multiplied with the interacting Lagrangian, i.e.

$$
S[\phi]=\int_{-\infty}^{\infty} d t \int d^{3} x\left\{\mathcal{L}_{0}[\phi]+\theta\left(t-t_{\text {in }}\right) \mathcal{L}_{\text {int }}[\phi]\right\} .
$$

In this perspective the non-local periodic terms that appear in the two- and fourpoint function should disappear in the limit where the interaction is always switched-on as in the traditional in-out approach. We remark that this limit cannot be taken by 
simply sending $t-t_{\text {in }} \rightarrow \infty$ because it is not defined for our oscillatory terms.

In Section 6.1 we discuss the profile dependence of the two-point correlation function in different backgrounds in order to understand the dependence of our results on the initial time. In Section 6.2 the issue how to properly define the adiabatic limit to recover the Poincaré invariance in Minkowski spacetime is discussed.

\subsection{Profile dependence of the two-point correla- tion function}

In the following we first study the interaction profile dependence of our results for the two-point function both in Minkowski and de-Sitter spacetime. We first construct an interaction profile that mimics the properties of a nearly adiabatic switching-on of the interaction. Then we will use it in order to better understand the finite time dependence of our results.

We are interested in a function $g$ with regularity $\mathcal{C}^{n}(\mathbb{R})$ that is 0 before the initial time $t<t_{\text {in }}$ and 1 after the transition, i.e. for $t>t_{\text {in }}+\Delta t$. Here $\Delta t$ is a parameter that represents the transition time. Let $f$ be a function $\mathcal{C}^{n}(\mathbb{R})$ at 0 and $f(0)=0$, then

$$
g(t)=\frac{f\left(t-t_{\text {in }}\right)}{f\left(t-t_{\text {in }}\right)+f\left(\Delta t-t+t_{\text {in }}\right)}
$$

is the interacting profile that we were looking for. The function $f$ can be chosen to be $x \mapsto x^{n+1}$ or $x \mapsto e^{-1 / x^{2}}$ in order to get a $\mathcal{C}^{n}$ or $\mathcal{C}^{\infty}$-extension of the interaction. We show few examples in Figure 6.1.

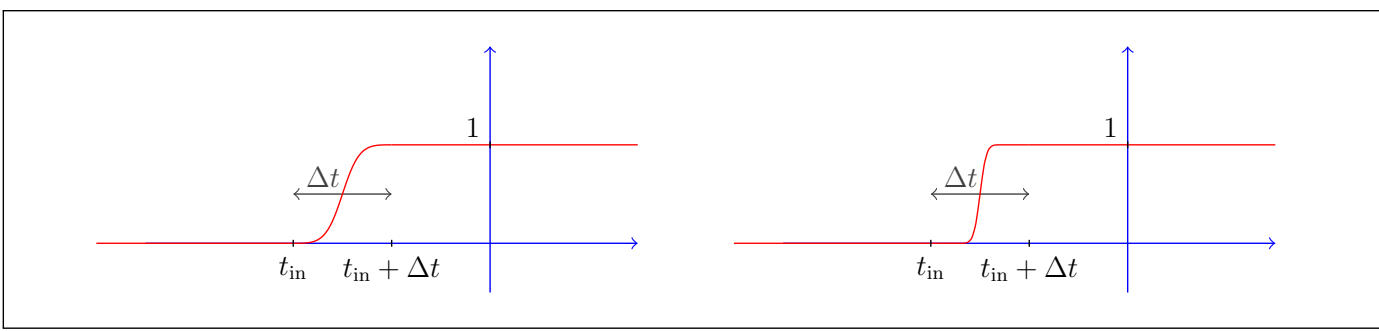

Figure 6.1: Interaction profiles with $\mathcal{C}^{2}(\mathbb{R})$ and $\mathcal{C}^{\infty}(\mathbb{R})$ extensions during the transition

It is important to notice that for small $\Delta t$, a less regular switching-on of the interaction should be preferred for two main reasons. First, less regular functions are simpler to treat analytically. Secondly, functions that are too regular tend to stay constant after the initial time and before the transition, giving us extensions that are similar to the $\theta$-profile. 


\section{Profile-dependence of the tadpole in Minkowski spacetime}

In the following we compute the regulated amplitude for a linear profile, i.e. $f: t \mapsto t$. First we remind the time dependence of the renormalized two-point function in Minkowski spacetime (5.11)

$$
\lambda \frac{\sin \left(w_{k}\left(t-t_{\text {in }}\right)\right)^{2}}{2 w_{k}^{3}},
$$

where $w_{k}=\sqrt{k^{2}+m^{2}}$. Now, by replacing $\lambda$ with the new time-dependent coupling constant $\lambda(t)=\lambda g(t)$, the new dependence on the external propagators can be computed. We found that before the transition the time dependence of the amplitude becomes proportional to

$$
\frac{\lambda}{8} \frac{2 w_{k}\left(t-t_{\text {in }}\right)-\sin \left(2 w_{k}\left(t-t_{\text {in }}\right)\right)}{\Delta t w_{k}^{4}}, \quad t<t_{\text {in }}+\Delta t
$$

and after the transition to

$$
\frac{\lambda}{8}\left[\frac{2}{w_{k}^{3}}+\frac{\sin \left(2 w_{k}\left(t-t_{\text {in }}-\Delta t\right)\right)-\sin \left(2 w_{k}\left(t-t_{\text {in }}\right)\right)}{\Delta t w_{k}^{4}}\right], \quad t>t_{\text {in }}+\Delta t .
$$

In other words the first expression represents the amplitude during the transient region and the second one represents the analogous of the periodic oscillations that were found in (5.11). The last expression has a finite time-independent limit for large transition time $\Delta t$. It is given by

$$
\frac{\lambda}{4 w_{k}^{3}}
$$

In position space this term becomes

$$
\mathcal{F}^{-1}(\cdot)=4 \pi \int_{0}^{\infty} d k \frac{\sin (k r)}{k r} k^{2} \frac{\lambda}{4 w_{k}^{3}}=\lambda \pi K_{0}(m r)
$$

where $K_{0}$ is the modified Bessel function of second kind.

Finally, the limit $\Delta t \rightarrow 0$ consistently gives us the contribution of the $\theta\left(t-t_{\text {in }}\right)$ function

$$
\lambda \frac{\sin \left(w_{k}\left(t-t_{\text {in }}\right)\right)^{2}}{2 w_{k}^{3}} .
$$

In Figure 6.2 we show the renormalized two-point function with an interaction profile $g(t)$ constructed from a quadratic and cubic function $f$. We see that using the construction in eq. (6.2), the oscillations are always present and they are suppressed by the transition time $\Delta t$. For a longer transition time we found a smaller amplitude of the oscillation. The amplitude and the form of the oscillations clearly depend also on the chosen switching-on profile. It should be noted that the constant contribution 


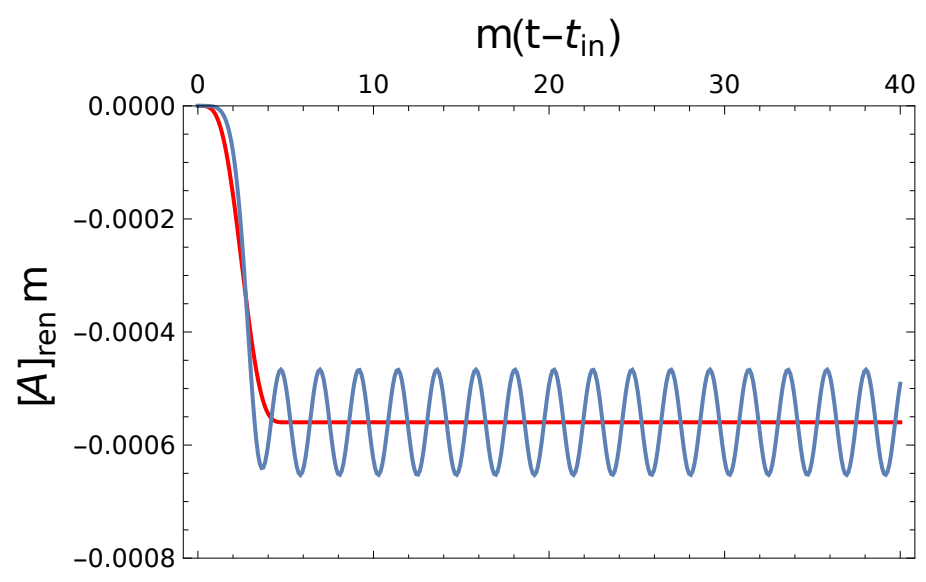

Figure 6.2: Renormalized two-point function using different continuous interaction profiles $\lambda(t)$ where $\lambda\left(t-t_{\text {in }}<0\right)=0$ and $\lambda\left(t-t_{\text {in }}>5 / m\right)=1$ for $k / m=1$ and $m /(2 \mu)=1$. The blue line corresponds to an interaction profile with a cubic behaviour about time 0 and before the transition. The red line corresponds to a quadratic switching-on.

that we obtain after suppressing the oscillations does not depend on the chosen profile and is in agreement with the values of the amplitude found in the literature [84] in the in-out approach.

It is interesting to test the profile dependence with other functions that do not depend on the previous construction. The most natural function that simulates the switching-on of an interaction is given by the arc-tangent shown in Figure 6.3.

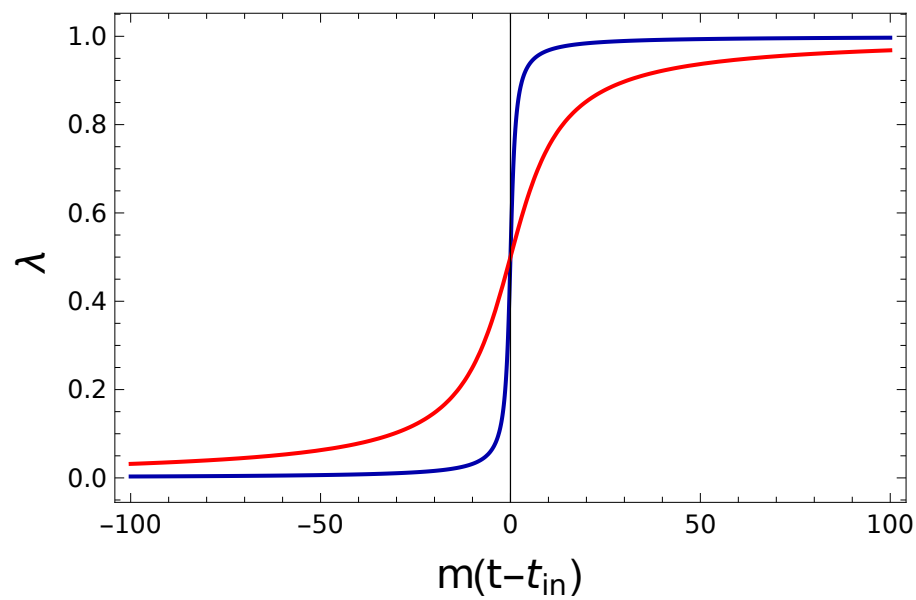

Figure 6.3: Switching-on profile constructed from the arc-tangent function with $y=0$, $x=1$ (blue line) or $x=10$ (red line). The coupling $\lambda$ is normalized to 1 .

Let's define the following interaction profile

$$
\lambda(t)=\frac{\lambda}{\pi}\left(\frac{\pi}{2}+\operatorname{atan}\left(x^{-1}\left(t-t_{\text {in }}\right)-y\right)\right),
$$


where $x$ is a parameter that quantify the width of the switching-on and $y$ is a parameter that identifies the jump at $t_{\text {in }}$.

The result is shown in Figure 6.4 and is similar to the previous simulations, with the difference that the tadpole seems to always depend on the initial time $t_{\text {in }}$ without a clear suppression of the oscillations. This is not surprising, because $\lambda(t) / \lambda$ is a function that is 1 (or 0 ) only for positive (or negative) infinite times. This means, that for a finite initial time $t_{\text {in }}$, there is always a jump that is quantified by $y$. In fact

$$
\lambda\left(t_{\text {in }}\right) / \lambda=\frac{1}{2}+\operatorname{atan}(y)
$$

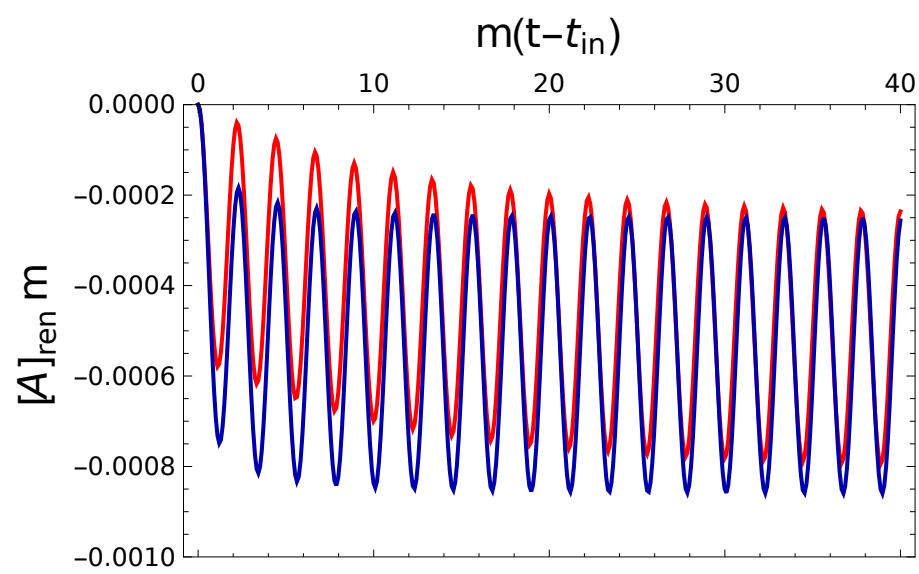

Figure 6.4: Renormalized two-point function using the arc-tangent interaction profile with $k / m=1$ and $m /(2 \mu)=1, y=0, x=1$ (blue line) or $x=10$ (red line).

From the previous considerations, we conclude that the time-dependent periodic features in the renormalized two-point function in Minkowski spacetime depend on the chosen interaction profile and are therefore a consequence of the fact that in the Schwinger and Keldysh formalism we are describing the physical situation of an interacting time evolution from an initial time where we suppose the system to be on the vacuum state of the free theory. The periodic features can be suppressed by taking an adiabatic switching-on of the interaction where we observed a residual constant contribution given by eq. (6.6).

\section{Profile dependence of the Feynman tadpole in Minkowski space- time}

In this thesis we calculated the renormalization of the correlation functions in the closedtime-path formalism, i.e. we calculated the radiative corrections to the correlation function of the field component $\phi^{(1)}$. The two-point function of the field $\phi^{(1)}$ is called 
the Hadamard or Schwinger propagator and it is related to the Feynamn propagator $G^{F}$. It is interesting to investigate the profile dependence for the tadpole amplitude obtained with Feynman propagators in order to have a direct comparison with the results in the literature for the in-out approach.

The Feynman propagator can be defined from the Schwinger and advanced/retarded propagators [134]

$$
G^{F}(x, y)=i\langle\mathrm{~T}\{\phi(x) \phi(y)\}\rangle=\frac{1}{2}\left(G^{R}+G^{A}\right)+i F
$$

We found that the difference between the radiative correction to the two-point function computed in the Schwinger and Keldysh formalism and the loop correction to the tadpole in the in-out approach is given by

$$
\begin{aligned}
\left\langle\mathrm{T}\left\{\phi\left(x, t_{a}\right) \phi\left(y, t_{b}\right)\right\}\right\rangle_{\mathrm{CTP}}^{(1)}=\left\langle\mathrm{T}\left\{\phi\left(x, t_{a}\right) \phi\left(y, t_{b}\right)\right\}\right\rangle_{\mathrm{STD}}^{(1)} & \\
& +\frac{\lambda m^{2} C}{64 \pi^{2} w_{k}^{3}} \cos \left(w_{k}\left(t_{a}+t_{b}-2 t_{\text {in }}\right)\right),
\end{aligned}
$$

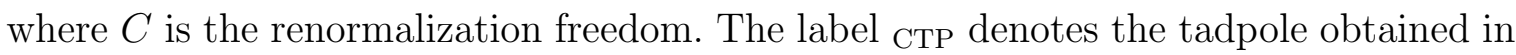
the Schwinger and Keldysh formalism and STD denotes the traditional in-out approach of quantum field theory.

We expect that with an adiabatic switching-on of the interaction the cosine function is strongly suppressed and the two tadpoles obtained with different approaches coincide. Indeed we found that using the profile construction described in (6.2), the amplitude of the oscillations given by the cosine function is suppressed to zero. In this case there is no residual constant contribution and we consistently recover the in-out result.

We tried to compute the analytic result using a linear switching-on function $f(x)=x$. We found that the contribution of the external Feynman propagators gives the standard result plus a periodic (non-covariant) contribution that becomes negligible for a large transition time $\Delta t$, as expected. The non-local contribution reads

$$
-\frac{i C \lambda}{64 \pi^{2} w_{k}^{4}}\left[\sin \left(w_{k}\left(t_{a}+t_{b}-2 t_{\text {in }}\right)\right)-\sin \left(w_{k}\left(t_{a}+t_{b}-2\left(t_{\text {in }}+\Delta t\right)\right)\right)\right] \frac{1}{\Delta t} .
$$

We remark that in the previous examples the constant residual of the oscillations after the adiabatic switching-on is exactly given by the midpoint of the oscillatory functions. Indeed we found a constant contribution of $1 / 2$ for the $\sin ^{2}$ function and 0 for the cosine function. 


\section{Profile-dependence of the tadpole in de-Sitter spacetime}

We are going to study the profile dependence of the renormalized two-point function in de-Sitter spacetime. As pointed-out in the introduction, here we do not expect only the finite time contributions arising from the external propagators but also an explicit time dependence originated from the background.

In the following we proceed with the same strategy used for a flat background. First we will present our analytic results by changing the interaction profile using the construction of eq. (6.2) for a linear auxiliary function $f: x \rightarrow x$. Unfortunately we were not able to extract an analytic result with the full expression (5.22) and we could only consider the limit where the external propagators are on scales $|k \tau| \ll 1$. To conclude we will show the full result obtained numerically with the full propagators.

We start by reminding the time dependence of the renormalized two-point correlation function in de-Sitter spacetime (5.24), where we considered only the first orders in $|k \tau|$ for the external propagators

$$
\frac{\lambda}{2}\left(\log \left(\frac{\tau}{\tau_{\text {in }}}\right)+\frac{1}{3}-\frac{\tau^{3}}{3 \tau_{\text {in }}^{3}}\right)
$$

Now, by replacing the coupling constant $\lambda$ with the time-dependent interaction profile $\lambda(t)=\lambda g(t)$ with a linear switching-on function $f: t \rightarrow t$, after the transition the following time dependence was found

$$
\begin{aligned}
& \frac{1}{6}\left[4+3 \log \left(\frac{\tau}{\Delta \tau}\right)\right. \\
& \left.\quad+\frac{3\left(\tau_{\text {in }} \log \left(\frac{\tau_{\text {in }}}{\tau_{\text {in }}+\Delta \tau}\right)+\Delta \tau \log \left(\frac{\Delta \tau}{\tau_{\text {in }}+\Delta \tau}\right)\right)}{\Delta \tau}+\frac{-\tau^{3}\left(2 \tau_{\text {in }}+\Delta \tau\right)}{2 \tau_{\text {in }}^{2}\left(\tau_{\text {in }}+\Delta \tau\right)^{2}}\right] .
\end{aligned}
$$

This result considers only the smallest order in $k \tau$ for the external propagators. As it can be noticed, the dependence on the initial time disappears for a large transition $\Delta \tau \gg 1$. The result is still time-dependent because of the manifest logarithmic timedependence. This is consistent with the idea that the logarithmic time dependence arises from the background and is not a consequence of the finite initial time of the theory.

By taking the limit $\Delta \tau \rightarrow 0$ we recover the result obtained with the $\theta$-profile. 
Indeed

$$
\begin{aligned}
& \frac{1}{6}\left[4+3 \log \left(\frac{\tau}{\Delta \tau}\right)+\frac{3\left(\tau_{\text {in }} \log \left(\frac{\tau_{\text {in }}}{\tau_{\text {in }}+\Delta \tau}\right)+\Delta \tau \log \left(\frac{\Delta \tau}{\tau_{\text {in }}+\Delta \tau}\right)\right)}{\Delta \tau}+\frac{-\tau^{3}\left(2 \tau_{\text {in }}+\Delta \tau\right)}{2 \tau_{\text {in }}^{2}\left(\tau_{\text {in }}+\Delta \tau\right)^{2}}\right] \\
& =\frac{1}{6}\left(4-3 \tau_{\text {in }} \frac{1}{\tau_{\text {in }}}+3 \log \left(\frac{\tau}{\Delta \tau}\right)+3 \log \left(\frac{\Delta \tau}{\tau_{\text {in }}}\right)+\frac{-2 \tau^{3} \tau_{\text {in }}}{2 \tau_{\text {in }}^{2} \tau_{\text {in }}^{2}}\right) \\
& =\frac{1}{6}\left(1+3 \log \left(\frac{\tau}{\tau_{\text {in }}}\right)+\frac{-\tau^{3}}{\tau_{\text {in }}^{3}}\right) .
\end{aligned}
$$

To conclude our discussion we show in Figure 6.5 the profile dependence of the renormalized tadpole in de-Sitter spacetime considering the massless expression for the external propagators (4.34).

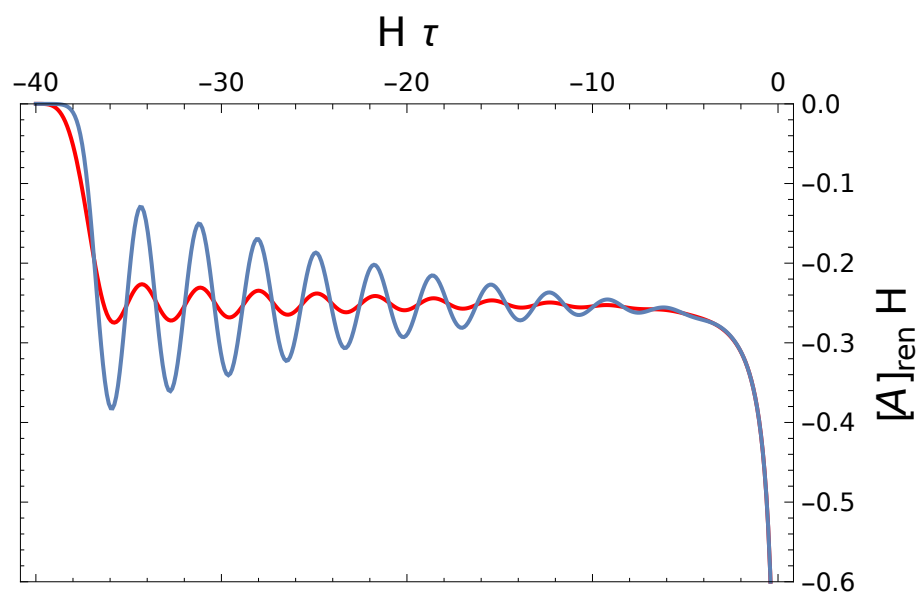

Figure 6.5: Interaction profile dependence of the renormalized tadpole diagram in de-Sitter spacetime where $\lambda\left(\tau-\tau_{\text {in }}<0\right)=0$ and $\lambda\left(\tau-\tau_{\text {in }}>5 / H\right)=1$ for $H \tau_{\text {in }}=-40$, $k / H=1, \varepsilon=1 /\left(16 \pi^{2}\right)$ and $H / \mu=1$. The blue line corresponds to an interaction profile with a quartic behaviour about time 0 and before the transition. The red line corresponds to a quadratic switching-on.

The result for different profiles should be compared to Figure 5.5, where we computed the two-point function with the usual $\theta$-profile. We see that the amplitude of the periodic features depends on the switching-on profile and is suppressed for large transition time. As in Minkowski spacetime we observe a residual constant contribution that does not depend on the switching-on of the interaction.

This concludes our analysis of the profile dependence of the two-point correlation function for different backgrounds. We found that for different profiles the oscillatory features that we found in Minkowski and de-Sitter spacetime depend on the chosen profile and that the initial time dependence is suppressed for an adiabatic switching-on. This is in agreement with the consideration that in Minkowski spacetime one should recover the Poincaré symmetry with an adiabatic switching-on of the interaction. 
On the other hand, in de-Sitter spacetime we expect also contributions arising from the time-dependent evolving background. This is indeed the case, we found that the logarithmic divergence that is expected to be a contribution coming from the geometry of the background is not suppressed with a different choice of the profile.

In the next section, we will approach the issue how to properly define the adiabatic limit in order to get the constant contribution directly from the oscillations.

\subsection{Proper definition of the adiabatic limit of the tadpole}

In the previous section we described the profile dependence of our results about the renormalization of the two-point correlation function in Minkowski and quasi de-Sitter spacetime. In Minkowski spacetime, we expect to recover the exact Poincaré covariance in the limit $t-t_{\text {in }} \rightarrow \infty$.

In eqs. (6.8) and (6.12) the time dependence of the tadpole propagator in Minkowski spacetime was described. It is clear that one cannot directly take the adiabatic limit $t-t_{\text {in }} \rightarrow \infty$ of these expressions because it is not well-defined. In other words, the limits $\lim _{t \rightarrow \infty} \sin (t)^{2}$ and $\lim _{t \rightarrow \infty} \cos (t)$ do not exist.

We will try to overcome this issue by treating the adiabatic limit in the language of distributions in time. Let's consider the following time-integral that represents the contribution coming from the external propagators

$$
\int_{t_{\text {in }}}^{\infty} d \tau\left(-i G^{R}(k, t, \tau)\right) F(k, \tau, t) .
$$

As discussed before one cannot directly take the limit $t_{\text {in }} \rightarrow-\infty$. This issue can be approached by considering the previous expression as a distribution in $\mathcal{D}(\mathbb{R})$, i.e.

$$
\int_{t_{\mathrm{in}}}^{\infty} d \tau\left(-i G^{R}(k, t, \tau)\right) F(k, \tau, t) f_{s}(\tau)
$$

where $f_{s}(\tau)$ is a family of test-functions that depend on a continuous parameter $s$. In this picture the adiabatic limit consists in the limit $f_{s}(t) \rightarrow 1$. Let's suppose that $f_{s}$ is a function that satisfies this property for $s \rightarrow \infty$. The adiabatic limit becomes

$$
\lim _{s \rightarrow \infty} \int_{-\infty}^{\infty} d \tau\left(-i G^{R}(k, t, \tau)\right) F(k, \tau, t) f_{s}(\tau)
$$

In order to be more quantitative, we consider the example where $f_{s}$ is given by the 
following family of functions (see Figure 6.6)

$$
f_{s}: \tau \rightarrow e^{\frac{-\tau^{2}}{s^{2}}}
$$

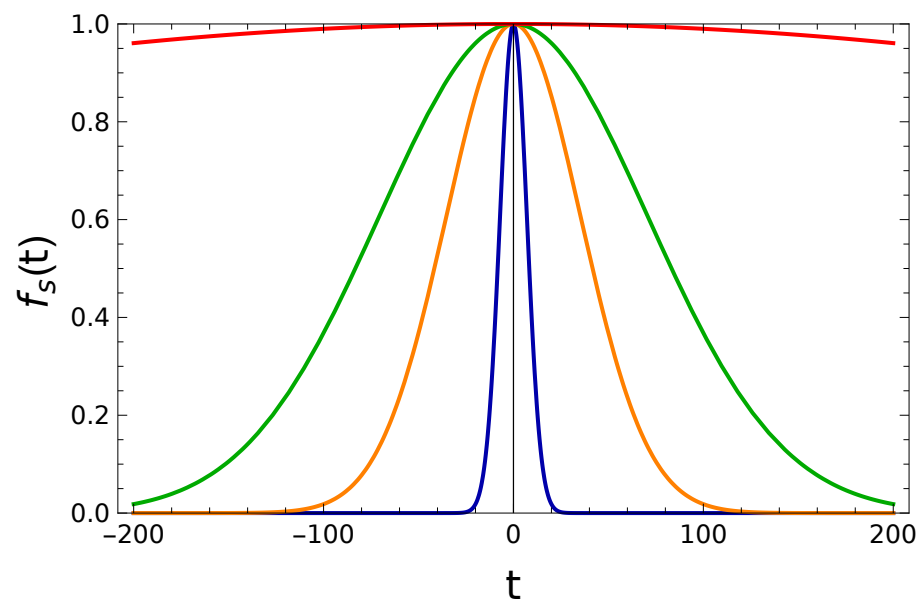

Figure 6.6: One-parameter family of test functions $f_{s}(t)=e^{\frac{-t^{2}}{s^{2}}}$ for different parameters: $s=10$ (blue line), $s=50$ (orange line), $s=100$ (green line) and $s=1000$ (red line).

In this case the limit of eq. (6.19) is well defined and does not depend on $\tau$, as expected. In Figures 6.7 and 6.8 we show the time-evolution of the tadpole amplitude for large values of $s$ and the continuous dependence of the amplitude on $s$ for a fixed time. In both cases we found that by increasing the parameter $s$, the amplitude of eq. (6.18) tends to a constant value consistent with the results obtained in the discussion of the profile dependence of the two-point function.

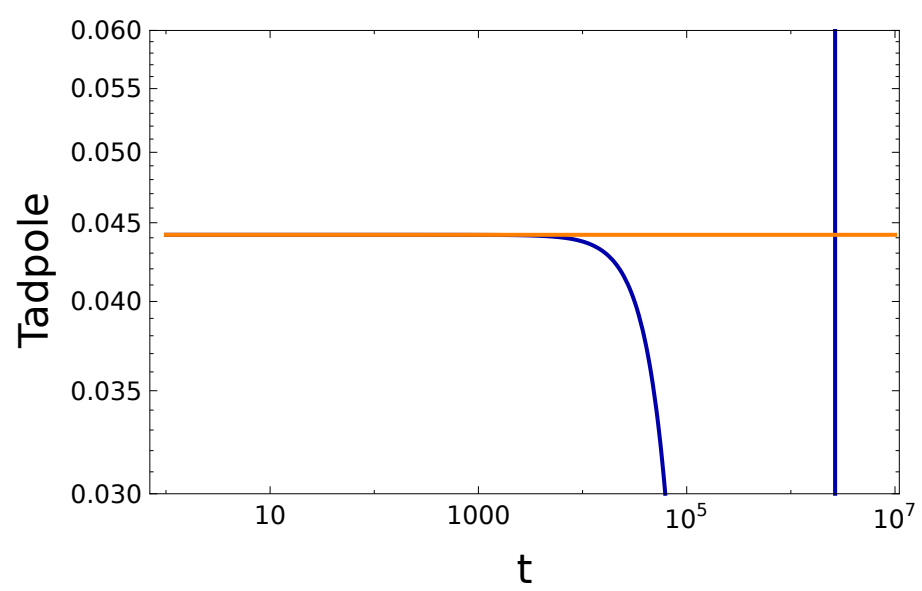

Figure 6.7: Time-evolution of the tadpole amplitude (blue line) to show the constant contribution (orange line) in the adiabatic limit. We fixed $s=10^{5}$.

This concludes our discussion of the interaction profile and of the proper definition of the adiabatic limit for the amplitudes of the two-point correlation function. By 


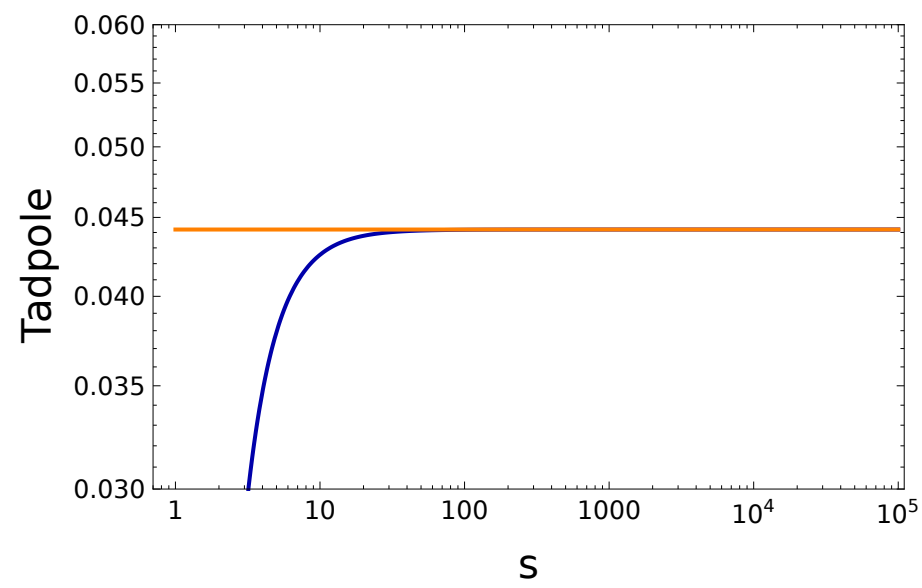

Figure 6.8: Tadpole amplitude (blue line) for different parameters $s$ to show the constant contribution (orange line) in the adiabatic limit. We fixed $\tau=2$.

approaching the limit of the external propagators in the language of distributions we were able to define the adiabatic limit in a consistent way and to recover the constant contributions found when we discussed the interaction switching-on profiles with a large transition time. This discussion is needed in order to explain why we were not recovering the Poincaré covariance in the limit where the interaction is always on. We remark that in the next chapter we will discuss the physical situation where the system is explicitly set to the Bunch-Davies vacuum at the beginning of inflation. We will therefore use the Schwinger and Keldysh formalism without changing the interaction profile and without taking the adiabatic limit, because this would change the physics and would give a different description of the system. 


\section{CHAPTER 7

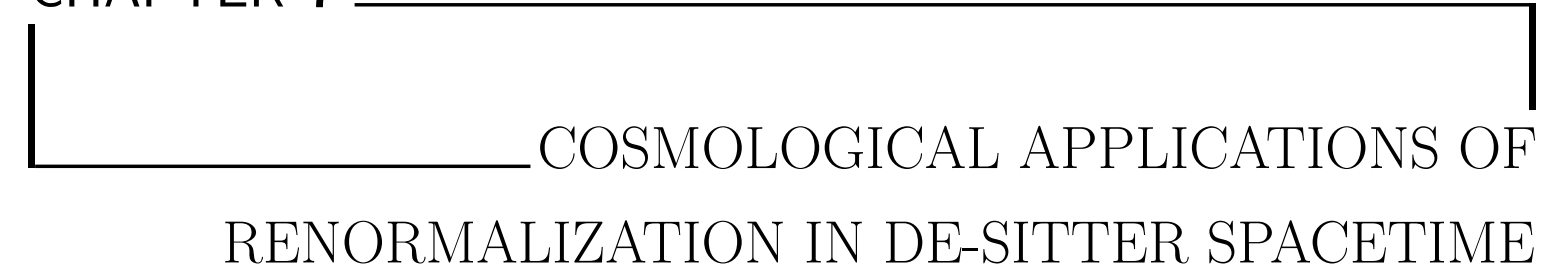

In this chapter the results obtained in the renormalization of the two- and four-point correlation functions in quasi de-Sitter spacetime are applied to cosmology in order to study the physical importance of radiative corrections in the primordial universe. The main goal is to estimate the effects of loop corrections to observable quantities such as the power spectrum and the non-linearity parameter $\tau_{\mathrm{NL}}$ for different models.

In Section 7.1 the classical dynamics of the inflationary models of interest is introduced. In particular the details of monomial inflation with a quartic interaction (Section 7.3) and the models inspired by hybrid inflation (Section 7.4) are discussed. In Section 7.2 the primordial spectrum of the inflaton field is analyzed in the in-in formalism in order to have a theoretical framework where the power spectrum can be treated perturbatively. At the end of the chapter, in Section 7.5, the effects of the time-dependent contributions to the trispectrum arising at tree-level are discussed. In particular we give our predictions for the non-linearity parameter $\tau_{\mathrm{NL}}$ which is an important cosmological observable describing the non-Gaussian nature of the primordial power spectrum.

\subsection{Classical dynamics of the inflationary models}

In this section the classical dynamics of different inflationary models is discussed and the results obtained in Chapter 5 are directly applied in order to compute and estimate the influence of radiative corrections to the power spectrum and trispectrum.

We first consider a simple model of a scalar field theory with monomial selfinteraction in curved spacetime with a canonical kinetic term and potential $V(\phi)$. The 
Lagrangian density reads

$$
\mathcal{L}[\phi]=\sqrt{-g}\left(\frac{1}{2} \partial_{\mu} \phi \partial^{\mu} \phi-\frac{1}{2} m^{2} \phi^{2}-V(\phi)+\frac{\xi}{2} \mathcal{R} \phi^{2}\right)+\delta \mathcal{L}
$$

where $g_{\mu \nu}$ is the metric describing the background with signature $(+---), g=\operatorname{det}\left(g_{\mu \nu}\right)$ is the determinant, and $\delta \mathcal{L}$ are the counter-terms

$$
\delta \mathcal{L}=\sqrt{-g}\left(\frac{1}{2} \delta Z \partial_{\mu} \phi \partial^{\mu} \phi-\frac{1}{2} \delta m^{2} \phi^{2}-\delta V(\phi)+\frac{\delta \xi}{2} \mathcal{R} \phi^{2}\right)
$$

In the following we suppose that the curvature coupling is vanishing at-tree-level, i.e. we will neglect the term proportional to $\xi \mathcal{R}$ in the Lagrangian (7.1) supposing that it does not affect the classical dynamics ${ }^{1}$. Nevertheless in Section 5.1 we found that a contribution $\delta \xi$ will be generated at one-loop level.

A period of slow-roll inflation is possible if the inflaton potential $V(\phi)$ fulfills the slow-roll conditions [7]

$$
\epsilon=\frac{M_{P}^{2}}{2}\left(\frac{V^{\prime}}{V}\right)^{2} \ll 1, \quad|\eta|=M_{P}^{2}\left|\frac{V^{\prime \prime}}{V}\right| \ll 1,
$$

where $M_{P}=2.4 \times 10^{18} \mathrm{GeV}$ is the reduced Planck mass. During slow-roll the equation of motion of the classical value of the field is simplified to

$$
\ddot{\phi}+3 H \dot{\phi}+V^{\prime}(\phi) \sim 3 H \dot{\phi}+V^{\prime}(\phi)=0
$$

that implies

$$
\dot{\phi}=-\frac{V^{\prime}(\phi)}{3 H} .
$$

The second FRW equation can also be simplified in the slow-roll regime and reads

$$
H^{2}=\frac{V(\phi)}{3 M_{P}^{2}}
$$

The number of e-folds from the value of the classical field $\phi$ to the one at the end of inflation $\phi_{\text {end }}$ is given by

$$
\begin{aligned}
N(\phi) & =\int_{t}^{t_{\mathrm{end}}} d t H(t)=\int_{\phi}^{\phi_{\mathrm{end}}} d \phi \frac{d t}{d \phi} H^{2}\left(\frac{1}{H}\right) \\
& =\int_{\phi_{\mathrm{end}}}^{\phi} d \phi \frac{d t}{d \phi}\left(\frac{V(\phi)}{3 M_{P}^{2}}\right)\left(\frac{3 \dot{\phi}}{V^{\prime}(\phi)}\right)=\frac{1}{M_{P}^{2}} \int_{\phi_{\mathrm{end}}}^{\phi} d \varphi \frac{V(\varphi)}{V^{\prime}(\varphi)},
\end{aligned}
$$

\footnotetext{
${ }^{1}$ This is not true for example for Higgs inflation [146].
} 
where in the last line we used the slow-roll equations (7.4) and (7.5).

In the standard scenario of inflation the quantum fluctuations of the inflaton field $\delta \phi$ are responsible for the primordial curvature perturbations $\delta \mathcal{R}$ and they can be directly related to late time observables as the temperature anisotropies in the cosmic microwave background or the observed large scale structure of the universe.

In the slow roll approximation, the power spectrum of the curvature perturbations could be expressed in terms of the inflaton potential $V(\phi)$ as [80]

$$
\mathcal{P}_{\mathcal{R}}(k)=\left.\frac{1}{12 \pi^{2} M_{P}^{6}} \frac{V^{3}}{V^{\prime 2}}\right|_{k=a H}
$$

where the potential $V(\phi)$ and its first derivative $V^{\prime}(\phi)$ are evaluated at the horizon exit $^{2}$, i.e. the scales $k$ are set to $k=a(t) H$. One can connect the comoving scale $k$ at horizon exit and the corresponding value of the classical inflaton through the number of e-folds using eq. (7.6) to get

$$
\log \left(\frac{k_{\mathrm{end}}}{k}\right)=\log \left(\frac{a\left(t_{\mathrm{end}}\right) H\left(t_{\mathrm{end}}\right)}{a\left(t_{k}\right) H\left(t_{k}\right)}\right) \sim N(\phi(k)),
$$

where $k_{\text {end }}$ is the comoving scale leaving the horizon at the end of slow-roll inflation. We assumed $H\left(t_{\text {end }}\right) \sim H\left(t_{k}\right)$. From the previous relations one can express the spectral index $n_{s}$ as a function of the slow-roll parameters at first-order to be

$$
n_{s}(k)-1=\frac{d \mathcal{P}_{\mathcal{R}}}{d \ln k}=2 \eta(\phi(k))-6 \epsilon(\phi(k))
$$

\section{Chaotic inflationary scenario}

The simplest class of models that realizes slow-roll inflation is given by the class of large field models where the scalar field is typically displaced from the minimum of the potential by a quantity of the order of the Planck mass. In the following we will discuss one of the most studied inflationary models consisting of a scalar field with monomial quartic self-interaction

$$
V(\phi)=\frac{\lambda}{4 !} \phi^{4}
$$

In the chaotic scenario the universe is assumed to emerge from a quantum gravitational energy density comparable to the Planck density. Therefore, because of the large friction term in the Friedmann equations, the field is slowly rolling down the potential. Inflation ends when the field is of the order of the Planck scale. This can be

\footnotetext{
${ }^{2}$ The curvature perturbation $\mathcal{R}$ freezes $\left(\dot{\mathcal{R}}_{k} \approx 0\right)$ once the mode crosses the horizon.
} 
seen by explicitly expressing the slow-roll parameters in terms of the classical value of the inflaton field $\phi$

$$
\begin{aligned}
& \epsilon=\frac{M_{P}^{2}}{2}\left(\frac{\frac{\lambda}{6} \phi^{3}}{\frac{\lambda}{24} \phi^{4}}\right)^{2}=\frac{M_{P}^{2}}{2} \frac{16}{\phi^{2}}=\frac{8 M_{P}^{2}}{\phi^{2}} \ll 1, \\
& \eta=M_{P}^{2}\left(\frac{\frac{\lambda}{2} \phi^{2}}{\frac{\lambda}{24} \phi^{4}}\right)=\frac{12 M_{P}^{2}}{\phi^{2}}=\frac{3}{2} \epsilon \ll 1,
\end{aligned}
$$

giving directly

$$
n_{s}(k)-1=2 \eta-6 \epsilon=-3 \epsilon=-\frac{24 M_{P}^{2}}{\phi^{2}}=-\frac{3}{N(k)},
$$

where the number of e-folds $N(k)$ was estimated from eq. (7.6)

$$
N(k)=\frac{1}{M_{P}^{2}} \int^{\phi} d \varphi\left(\frac{\frac{\lambda}{24} \varphi^{4}}{\frac{\lambda}{6} \varphi^{3}}\right)=\frac{1}{8 M_{P}^{2}} \phi^{2} .
$$

Because the only parameter in the model is the self-coupling $\lambda$, this will directly fix the prediction of the normalization of the power spectrum. In fact

$$
\begin{aligned}
\mathcal{P}_{\mathcal{R}}\left(k_{*}\right) & =\left.\frac{1}{12 \pi^{2} M_{P}^{6}} \frac{V^{3}}{V^{\prime 2}}\right|_{k_{*}=a H} \\
& =\frac{1}{12 \pi^{2} M_{P}^{6}}\left(\frac{\left(\frac{\lambda}{24} \phi_{*}^{4}\right)^{3}}{\left(\frac{\lambda}{6} \phi_{*}^{3}\right)^{2}}\right) \\
& =\frac{1}{12 \pi^{2} M_{P}^{6}} \frac{\lambda \phi_{*}^{6}}{384} \\
& =\frac{\lambda \phi_{*}^{6}}{4608 \pi^{2} M_{P}^{6}}=\frac{\lambda N_{*}^{3}}{9 \pi^{2}},
\end{aligned}
$$

where $N_{*}=3 /\left(1-n_{s}\left(k_{*}\right)\right)$ and $k_{*}$ is the pivot scale giving

$$
\lambda=\left(\frac{9 \pi^{2}}{N_{*}^{3}}\right) \mathcal{P}_{\mathcal{R}}\left(k_{*}\right)=\frac{\pi^{2}}{3}\left(1-n_{s}\left(k_{*}\right)\right)^{3} \mathcal{P}_{\mathcal{R}}\left(k_{*}\right)=2.97 \times 10^{-13},
$$

for the observed values of $\mathcal{P}_{\mathcal{R}}\left(k_{*}\right)=A_{s}=(2.20 \pm 0.08) \times 10^{-9}$ and $n_{s}\left(k_{*}\right)=0.9655 \pm$ 0.0062 [6]. In this class of models the normalization of the power spectrum imposes very small couplings, i.e. very flat inflaton potential in order to be able to generate the observable fluctuations. It should be noted that the $\lambda \phi^{4}$ model is excluded by the current observation because it lies outside the $99.7 \%$ confidence level to be consistent with the latest Planck data [147]. In fact the scalar-to tensor ratio $r=16 / N$ is too 
large with respect to the predicted value. Nevertheless it is interesting to study the properties of the radiative corrections to this simple case since it is one of the most studied models of inflation.

The large suppression of the coupling that we found in the chaotic scenario will also kill all the effects of the one-loop radiative contributions because, as we have shown in Section 5.1, the first order corrections are proportional to the coupling $\lambda$.

\section{Models inspired by the hybrid scenario}

In order to enhance the radiative effects on the classical dynamics of the inflaton field, the class of models that include more than one scalar field is considered. Indeed, in the case of the two-scalar fields hybrid model that we will discuss in the following, much larger couplings are allowed. The simplest version of this model is given by the effective potential

$$
V(\phi, \sigma)=\frac{m^{2}}{2} \phi^{2}+\frac{1}{4 g}\left(M^{2}-g \sigma^{2}\right)^{2}+\lambda_{h}^{2} \phi^{2} \sigma^{2}+\Delta V_{C W}(\phi)
$$

where $\Delta V_{C W}(\phi)$ is the one-loop correction to the effective potential $\grave{a}$ la ColemanWeinberg [148]

$$
\Delta V_{C W}(\phi)=\sum_{i}(-1)^{F} \frac{m_{i, \mathrm{eff}}^{4}(\phi)}{64 \pi^{2}}\left(\ln \left(\frac{m_{i, \mathrm{eff}}^{2}(\phi)}{\mu^{2}}\right)-\frac{3}{2}\right)
$$

where $(-1)^{F}$ gives a minus sign for fermionic fields and a positive sign for bosonic fields, the sum runs over all the fields of the theory and $\mu$ is an arbitrary renormalization energy scale.

For the considered potential, in the case of $\sigma=0$, its effective mass is given by $m_{\sigma}=2 \lambda_{h}^{2} \phi^{2}-M^{2}$ and the effective mass of the inflaton field $\phi$ is $m^{2}$. Therefore we have

$$
\Delta V_{C W}(\phi)=\frac{\left(2 \lambda_{h}^{2} \phi^{2}-M^{2}\right)^{2}}{64 \pi^{2}}\left(\ln \left(\frac{2 \lambda_{h}^{2} \phi^{2}-M^{2}}{\mu^{2}}\right)-\frac{3}{2}\right)+\frac{m^{4}}{64 \pi^{2}}\left(\ln \left(\frac{m^{2}}{\mu^{2}}\right)-\frac{3}{2}\right) .
$$

The last term is constant and does not contribute to the dynamics as long as the field $\sigma$ is vanishing. 
If the inflaton mass dominates the slope of the potential one has

$$
\begin{aligned}
& \epsilon=\frac{M_{P}^{2}}{2}\left(\frac{m^{2} \phi}{\frac{m^{2}}{2} \phi^{2}}\right)^{2}=\frac{M_{P}^{2}}{2} \frac{4}{\phi^{2}}=\frac{2 M_{P}^{2}}{\phi^{2}} \ll 1, \\
& \eta=M_{P}^{2}\left(\frac{m^{2}}{\frac{m^{2}}{2} \phi^{2}}\right)=\frac{2 M_{P}^{2}}{\phi^{2}}=\epsilon \ll 1 .
\end{aligned}
$$

The comoving scale $k$ at horizon exit is related to the corresponding value of the classical inflaton field by

$$
n_{s}(k)-1=2 \eta-6 \epsilon=-4 \epsilon=-\frac{8 M_{P}^{2}}{\phi^{2}(k)}=-\frac{2}{N(k)},
$$

where we used the number of e-folds given by

$$
N(k)=\frac{1}{M_{P}^{2}} \int^{\phi} d \varphi\left(\frac{\frac{m^{2}}{2} \varphi^{2}}{m^{2} \varphi}\right)=\frac{1}{4 M_{P}^{2}} \phi^{2} .
$$

The normalization of the power spectrum reads

$$
\begin{aligned}
\mathcal{P}_{\mathcal{R}}\left(k_{*}\right) & =\left.\frac{1}{12 \pi^{2} M_{P}^{6}} \frac{V^{3}}{V^{\prime 2}}\right|_{k_{*}=a H} \\
& =\frac{1}{12 \pi^{2} M_{P}^{6}}\left(\frac{\left(\frac{m^{2}}{2} \phi_{*}^{2}\right)^{3}}{m^{4} \phi_{*}^{2}}\right) \\
& =\frac{1}{12 \pi^{2} M_{P}^{6}} \frac{m^{2} \phi_{*}^{4}}{8} \\
& =\frac{m^{2} \phi_{*}^{4}}{96 \pi^{2} M_{P}^{6}}=\frac{m^{2} N_{*}^{2}}{6 \pi^{2} M_{P}^{2}}
\end{aligned}
$$

and imposes the constraint

$$
\frac{m^{2}}{M_{P}^{2}}=\left(\frac{6 \pi^{2}}{N_{*}^{2}}\right) \mathcal{P}_{\mathcal{R}}\left(k_{*}\right)=\frac{3 \pi^{2}}{2}\left(1-n_{s}\left(k_{*}\right)\right)^{2} \mathcal{P}_{\mathcal{R}}\left(k_{*}\right)=0.38 \times 10^{-10}
$$

giving a value for the inflaton mass of $m=6.22 \times 10^{-6} M_{P}$ that has to be compared to

$$
H^{2}=\frac{1}{3 M_{P}^{2}} \frac{m^{2}}{2} \phi_{*}^{2}=\frac{2}{3} N\left(k_{*}\right) m^{2}>m^{2}
$$

In the case of a vanishing inflaton mass $m \sim 0$, the slope can be provided by the radiative corrections related to the coupling $\lambda_{h}^{2}$. By comparing the first derivative of 
the potential, the one-loop corrections dominate for

$$
\frac{\lambda_{h}^{4} \phi^{3}}{4 \pi^{2}}>m^{2} \phi \quad \Leftrightarrow \quad \lambda_{h}^{4} \phi^{2}>4 m^{2} \pi^{2}
$$

which means a quartic coupling larger than

$$
\lambda_{h}^{2} \geq 2 \pi \frac{m}{\phi}
$$

The models of inflation where quantum corrections dominate the inflationary potential have been studied especially in supersymmetry $[149,150]$ and supergravity [151]. In these cases one can obtain a much larger value for the quartic coupling, of the order of $\lambda_{h} \sim 0.001$. We will use this as a maximal value in order to enhance the radiative effects.

Motivated by the large value of the coupling, we will discuss the dynamics of a hybrid model inspired by supersymmetry described by the following approximated scalar potential [151]

$$
\begin{aligned}
V & =\lambda_{h}^{2}\left|M_{G}^{2}-\Sigma^{2}\right|^{2}+4 \lambda_{h}^{2}|\Phi|^{2}|\Sigma|^{2}+M_{S}^{4}+\Delta V_{C W} \\
& =\lambda_{h}^{2} M_{G}^{4}+\frac{\lambda_{h}^{4} M_{G}^{4}}{8 \pi^{2}}\left[\ln \left(\frac{2 \lambda_{h}^{2} \phi^{2}}{\mu^{2}}\right)+\mathcal{O}\left(\frac{M_{G}^{4}}{\phi^{4}}\right)\right],
\end{aligned}
$$

where $\Phi, \Sigma$ are complex scalar fields, $M_{S}$ is the supersymmetry breaking scale and $1 / \sqrt{2} \phi$ denotes the real part of $\Phi$. In the last line we assumed $\Sigma=0$ in order to obtain the potential during inflation. The slow-roll parameters can be derived from the potential $V$ in eq. (7.30)

$$
\begin{gathered}
\epsilon=\frac{M_{P}^{2}}{2}\left(\frac{\frac{\lambda_{h}^{4} M_{G}^{4}}{8 \pi^{2}} \frac{2}{\phi}}{\lambda_{h}^{2} M_{G}^{4}}\right)^{2}=\frac{\lambda_{h}^{4}}{32 \pi^{4}} \frac{M_{P}^{2}}{\phi^{2}}, \\
\eta=M_{P}^{2}\left(\frac{-\frac{\lambda_{h}^{4} M_{G}^{4}}{8 \pi^{2}} \frac{2}{\phi^{2}}}{\lambda_{h}^{2} M_{G}^{4}}\right)=-\frac{\lambda_{h}^{2}}{4 \pi^{2}} \frac{M_{P}^{2}}{\phi^{2}} .
\end{gathered}
$$

They are both small for small couplings $\lambda_{h}$ and large value of $\phi$. As we did for the previous models we can express the spectral index, the number of e-folds and the normalization of the power spectrum in terms of the parameters of the model. The spectral index reads

$$
n_{s}(\phi)-1=2 \eta=-\frac{\lambda_{h}^{2}}{2 \pi^{2}} \frac{M_{P}^{2}}{\phi^{2}}=-\left(N+\frac{2 \pi^{2}}{\lambda_{h}^{2}} \frac{M_{G}^{2}}{M_{P}^{2}}\right)^{-1}
$$


where we used that the number of e-folds is given by

$$
N(k)=\frac{1}{M_{P}^{2}} \int_{\phi_{c}}^{\phi} d \varphi\left(\frac{\lambda_{h}^{2} M_{G}^{4}}{\frac{\lambda_{h}^{4} M_{G}^{4}}{8 \pi^{2}} \frac{2}{\varphi}}\right)=\frac{2 \pi^{2}}{\lambda_{h}^{2}}\left(\frac{\phi^{2}}{M_{P}^{2}}-\frac{\phi_{c}^{2}}{M_{P}^{2}}\right) .
$$

In the region of large $\lambda_{h}$, which is the region where the loop-corrections are enhanced, the normalization of the power spectrum fixes the scale $M_{G}$

$$
\begin{aligned}
\mathcal{P}_{\mathcal{R}}\left(k_{*}\right) & =\left.\frac{1}{12 \pi^{2} M_{P}^{6}} \frac{V^{3}}{V^{\prime 2}}\right|_{k_{*}=a H} \\
& =\frac{1}{12 \pi^{2} M_{P}^{6}}\left(\frac{\left(\lambda_{h}^{2} M_{G}^{4}\right)^{3}}{\left(\frac{\lambda_{h}^{4} M_{G}^{4}}{8 \pi^{2}} \frac{2}{\phi_{*}}\right)^{2}}\right) \\
& =\frac{4 \pi^{2} M_{G}^{4}}{3 \lambda_{h}^{2} M_{P}^{4}} \frac{\phi_{*}^{2}}{M_{P}^{2}} \\
& =\frac{4 \pi^{2} M_{G}^{4}}{3 \lambda_{h}^{2} M_{P}^{4}}\left(\frac{\lambda_{h}^{2} N_{*}}{2 \pi^{2}}+\frac{M_{G}^{2}}{M_{P}^{2}}\right) \\
& =\frac{2 N_{*} M_{G}^{4}}{3 M_{P}^{4}}+\frac{4 \pi^{2} M_{G}^{6}}{3 \lambda_{h}^{2} M_{P}^{6}},
\end{aligned}
$$

where the second term is negligible. Taking $N_{*}=1 /\left(1-n_{s}\left(k_{*}\right)\right)$ the scale $M_{G}$ is fixed to

$$
\frac{M_{G}}{M_{P}}=\left(\frac{3}{2}\left(1-n_{s}\left(k_{*}\right)\right) \mathcal{P}_{\mathcal{R}}\left(k_{*}\right)\right)^{1 / 4}=3.26 \times 10^{-3} .
$$

This value is comparable to the grand unification scale. We remark that for this model the spectral index (7.31) and the power spectrum normalization (7.33) do not depend on the coupling $\lambda_{h}$.

By requiring that the slow-roll conditions are satisfied up to the critical point $\phi_{c}=M_{G}$, from the slow-roll condition $|\eta|<0.1$ we can estimate the maximally allowed value of the coupling via

$$
|\eta|=\frac{\lambda_{h}^{2}}{4 \pi^{2}} \frac{M_{P}^{2}}{M_{G}^{2}}<0.1
$$

which means

$$
\lambda_{h}<2 \pi \frac{M_{G}}{\sqrt{10} M_{P}}=6.8 \times 10^{-3} .
$$

To conclude the discussion about two-scalar fields models inspired by hybrid inflation we discuss the example of a massive spectator field. The potential is similar to the hybrid model in eq. (7.17) but without any symmetry breaking in the spectator direction. By requiring that the classical dynamics is determined by the $m^{2} \phi^{2}$ term and not affected by the spectator field $\sigma$, the spectator's mass $m_{\sigma}$ has been found to be 
smaller than $m_{\sigma}<5 \times 10^{-2} M_{P}$ and the coupling constrained to be $\lambda_{h}^{2}<10^{-5}$ [152].

We remark that in this case the quartic coupling is smaller than in the hybrid case, but still larger than in the chaotic scenario. On the other hand the smallness of $\lambda_{h}$ is compensated by the value of $m_{\sigma}$ which can be larger.

In the last part of this chapter the radiative corrections to the power spectrum will be analyzed in the language of the Schwinger and Keldysh formalism. In Section 7.3 the radiative corrections to the primordial spectrum for the chaotic model are analyzed. Because of the smallness of the self-coupling, which is fixed by the normalization of the power spectrum we expect that the corrections are too small to be observed. In Section 7.4 we will investigate the time dependence of the one-loop corrections in the non-supersymmetric and supersymmetric hybrid model, and the spectator field model where we expect an enhancement of the radiative effect because of the larger coupling.

\subsection{Radiative corrections to the primordial power spectrum}

The power spectrum of primordial curvature perturbations is an important theoretical prediction that can be compared to cosmological observations. Because of its dependence on theoretical models, it becomes a powerful discriminator for distinguishing among different inflationary models. For example, from the primordial power spectrum one can predict the spectral index that is a measurement of the shape of the inflaton potential. This quantity can be directly compared to the observations. Unfortunately, for the investigated models, the loop corrections did not give any significant deviation to the tree-level prediction.

Nevertheless in Chapter 5 we found that the two-point correlation function gets radiative corrections with time-dependent oscillatory features. We expect to see the imprint of these oscillations in the one-loop corrections to the power spectrum. Periodic features are also predicted at tree-level for extended models [6, 19] or for non-standard initial states [153].

In the following, the power spectrum is defined in the theoretical framework of the Schwinger and Keldysh formalism. This will set the physical scenario where one can follow the time-evolution of observables including quantum effects arising from perturbation theory.

For a single scalar field inflationary model the power spectrum of the primordial fluctuations is directly connected to the two-point correlation function of the inflaton fluctuations. Let $\delta \phi(t, x)$ be the quantum fluctuations of the inflaton field $\phi(t)$. The Fourier transform of the two-point correlation function of the inflaton fluctuations 
defines the power spectrum $\mathcal{P}_{\delta \phi}$ in the following way

$$
(2 \pi)^{3} \delta^{(3)}\left(k+k^{\prime}\right) \mathcal{P}_{\delta \phi}(k)=\left\langle\delta \phi_{k} \delta \phi_{k^{\prime}}\right\rangle
$$

where $\delta \phi_{k}$ are the Fourier modes. In the language of the closed-time-path formalism the power spectrum is given by the time-evolution from an initial state where we assume the system to be in the Bunch-Davies vacuum $\rho_{B D}$

$$
\mathcal{P}_{\delta \phi}(k)=\left.\operatorname{Tr}\left\{\rho_{\mathrm{BD}} \mathrm{T}_{\mathcal{C}}\left[\left|\delta \phi_{k}\right|^{2} e^{-i \int_{t_{\mathrm{in}}}^{\infty} d \tau\left[\hat{H}_{I}^{+}(\tau)-\hat{H}_{I}^{-}(\tau)\right]}\right]\right\}\right|_{k=a H}
$$

where the horizon exit condition on time $k=a(t) H(t)$ implies that the spectrum is dependent only on the momentum variable $k$.

The in-in setup has the advantage that even if the Poincaré symmetry of the system is broken because of the time dependence of the background, one can still compute the radiative corrections. The perturbative expansion of the power spectrum has the following diagrammatic representation

$$
\mathcal{P}_{\delta \phi}(k)=\left(-\frac{0}{}+\ldots\right)
$$

What is typically extracted from data is the power spectrum of the curvature fluctuations that for single scalar field models has a simple relation to the power spectrum of the inflaton fluctuations

$$
\mathcal{P}_{\mathcal{R}}(k)=\frac{k^{3}}{4 \pi^{2}}\left(\frac{H^{2}}{\dot{\phi}^{2}}\right) \mathcal{P}_{\delta \phi}(k)=\frac{k^{3}}{4 \pi^{2}} \frac{1}{2 \epsilon M_{P}^{2}} \mathcal{P}_{\delta \phi}(k)
$$

where in the last equality the slow roll approximation is used and is equivalent to eq. (7.7). Because the power spectrum is evaluated at the horizon crossing, one expects to see the imprint of the early oscillations also in the primordial spectrum. We note that we assume this equality to be valid also for loop corrections, i.e.

$$
\mathcal{P}_{\mathcal{R}}(k)=\frac{k^{3}}{4 \pi^{2}} \frac{1}{2 \epsilon M_{P}^{2}}\left(\mathcal{P}_{\delta \phi}^{\text {tree }}(k)+\mathcal{P}_{\delta \phi}^{\text {loop }}(k)+\ldots\right)
$$

In principle the one-loop corrections for the scalar potential in eq. (7.18) should be included, but for simple monomial potentials they can be neglected [30].

In the following sections we will discuss the imprint in the power spectrum of the time-dependent oscillations that we found in the radiative corrections to the two-point 
function. The hope is that in some regime and for some particular inflationary models these effects become important and can become observable. The power spectrum becomes an interesting tool in order to test also the quantum nature of the primordial physics.

\subsection{One-loop corrections to the power spectrum with a monomial interaction}

In this section the one-loop corrections to the primordial power spectrum of the inflaton field is computed. The classical dynamics of the inflationary epoch was introduced in Section 7.1 and the radiative corrections to the power spectrum in Section 7.2. We will now apply the closed-time-path formalism in order to include the radiative corrections to the two-point correlation function computed in Chapter 5.

Because the correlation function is related to the primordial spectrum we expect to see the imprint of the early oscillations that arise from the initial conditions also in the power spectrum. The radiative corrections to $\mathcal{P}_{\mathcal{R}}$ can be included using the perturbative expansion in eq. (7.38). As we discussed previously the correction is proportional to the coupling constant $\lambda$, which is the only parameter of the theory and it is fixed to be very small from the spectrum normalization in eq. (7.16).

In Figure 7.1 we show our results for the one-loop correction to the power spectrum for different initial times where the loop contribution $P_{\mathcal{R}}^{\text {loop }}(k)$ is amplified by a factor $1.5 \times 10^{12}$ in order to be visible in the plot. Here the IR-cutoff is taken to the order of the slow-roll parameters $2 \varepsilon \sim 2 \eta=3 \epsilon$. For a quartic potential they are of order of $\mathcal{O}(0.1)$ and the effects of the infrared divergence are limited. In the literature other prescriptions found larger effects that could become visible for a sufficiently long inflationary phase. For example in [154] the infrared cutoff is the Hubble scale at the beginning of inflation and the IR correction depends on the duration of the inflationary phase [155]. For a long inflationary period, the oscillations are damped out and only the constant term contributes to the primordial spectrum. Since the main topic of this thesis is the study of the early oscillations, we will use the conservative choice of $\eta$ as the IR cutoff. It should be noted that the periodic behavior that we found at one-loop is different from the oscillations found for models that predict a feature-full power spectrum at tree-level (see [19] for a review of different models).

The power spectrum is plotted in a range of scales that are of physical interest today. We see that the largest effects are realized for large scales (small $k$ ). Unfortunately the spectral oscillations are suppressed for smaller scales. We remark that the one-loop corrections to the two-point function in eq. (5.22) describes the oscillations about a 

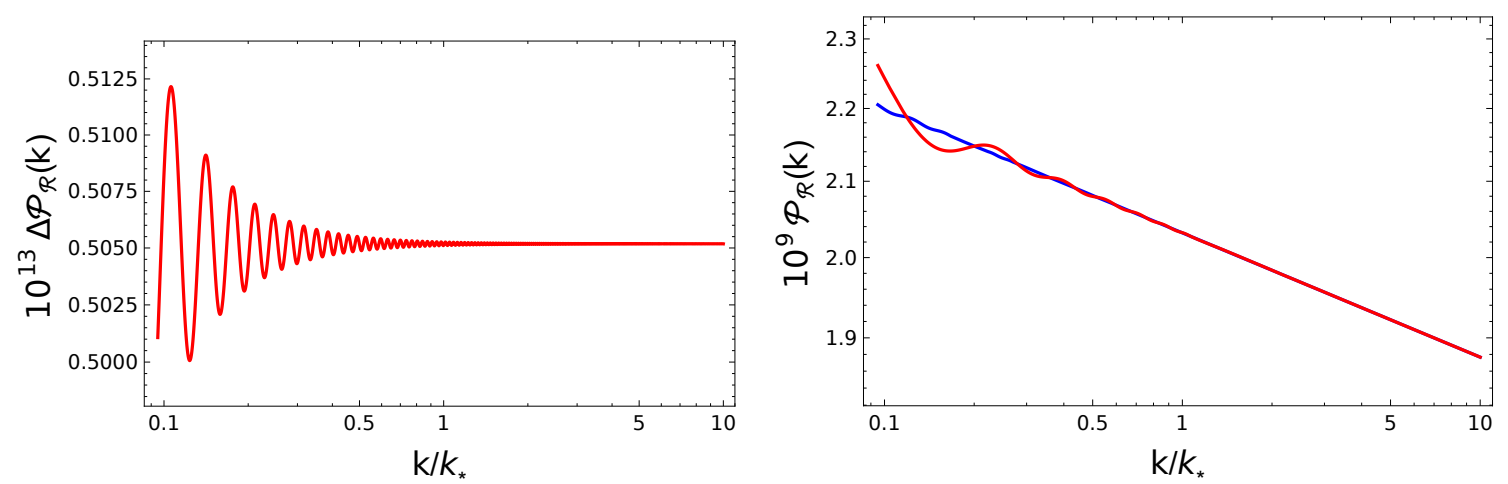

Figure 7.1: Relative difference and power spectrum for a scalar field theory with monomial interaction with $\lambda=2.97 \times 10^{-13}$. The renormalization scale was set to $\mu=10^{16} \mathrm{GeV}$ and the initial times to $\tau_{\text {in }}=-1 / k_{*} \exp \left(N_{\text {tot }}-N_{*}\right)$ with $N_{\text {tot }}=62$, $k_{*}=0.05 \mathrm{Mpc}^{-1}$ and $N_{*}=57.5$ (blue line) or $N_{*}=59$ (red line). In the right panel the corrections to the tree-level are amplified by a factor $1.5 \times 10^{12}$.

constant value independent from the initial time. This constant contribution gives a tiny shift in the primordial spectrum and can be reabsorbed into a redefinition of the coupling constant $\lambda$ giving an overall rescaling of the spectrum normalization. The shift is visible in the Figure 7.1 because it is enhanced by an artificial amplification factor of $1.5 \times 10^{12}$.

As expected the correction to the power spectrum is found to be very small and the order of magnitude is fixed by the smallness of the coupling $\lambda$. We found a departure from the tree-level power spectrum given by

$$
\left|\Delta \mathcal{P}_{\mathcal{R}}(k)\right|_{\text {quartic }}:=\left|\frac{\mathcal{P}_{\mathcal{R}}^{\text {loop }}(k)}{\mathcal{P}_{\mathcal{R}}^{\text {tree }}(k)}\right|_{\text {quartic }} \leq 0.5 \times 10^{-13}
$$

The correction is compatible with the current Planck observations [6] and it is too small to be observed even in future experiments. In the next section we consider a class of inflationary models inspired by hybrid inflation where the one-loop effects are enhanced because of the larger quartic coupling.

\subsection{One-loop corrections to the power spectrum in hybrid inflation}

In order to have a richer phenomenology the case of the hybrid inflationary model describing an inflaton field $\phi$ and a hybrid field $\sigma$ with the effective potential (7.17) is discussed. In this model one has to consider the loop corrections from the massive scalar field during inflation. 
The amputated tadpole is computed both using the WKB propagators and the hypergeometric function, in order to get the general solution renormalized with the counter-term found in the WKB regime. We found that the renormalized amplitude (5.67) has a quadratic dependence on the mass of the heavy field. This would enhance the effect of many orders of magnitude with respect to the chaotic scenario. In [29] they found that the IR modes are suppressed by negative powers of $a(\tau)$. This does not apply in the UV regime where the scale factor suppression of the WKB propagator is compensated by the scale factor dependence of the comoving cutoff [32] $\Lambda a(\tau)$. The techniques and the renormalization strategy is developed in details in Section 5.3.

In the following we discuss the one-loop contributions to the primordial spectrum for three models: the non-supersymmetric and supersymmetric hybrid inflation and quadratic inflation with a spectator field that does not influence the inflationary classical dynamics.

\section{Non-supersymmetric hybrid model}

The hybrid model described by the potential given in eq. (7.17) is the first example of radiative corrections to the primordial spectrum from a massive field. The classical dynamics was discussed in Section 7.1. In this case the mass that appears in the amputated amplitude in eq. (5.67) is the mass of the heavy field $m_{\sigma}^{2}=2 \lambda_{h}^{2} \phi^{2}-M^{2}$. If the mass term dominates the inflaton dynamics one has from eq. (7.22)

$$
m_{\sigma}^{2} \simeq 2 \lambda_{h}^{2} \phi^{2} \simeq 8 \lambda_{h}^{2} M_{P}^{2} N(k)
$$

where $N(k)$ is the e-folds number from the time where the scale $k$ leaves the horizon to the end of inflation. We remark that we have a slow adiabatic variation in the mass. Indeed

$$
\frac{\dot{w}_{k}}{w_{k}^{2}} \sim \frac{\dot{m}_{\sigma}}{m_{\sigma}^{2}}=\frac{1}{m_{\sigma}} \frac{\dot{N}}{2 N}=\frac{H}{2 m_{\sigma} N} \ll 1 .
$$

The renormalized WKB amputated amplitude (5.67) becomes

$$
\left[A_{\mathrm{amp}}\right]_{\mathrm{ren}}=\frac{-2 i \lambda_{h}^{4}}{\pi^{2}} \frac{N(k) M_{P}^{2}}{H^{4} \tau^{4}}\left(1+\log \left(\frac{\lambda_{h}^{2} M_{P}^{2} N(k)}{\mu^{2}}\right)\right) .
$$

The one-loop corrections to the power spectrum are given by

$$
\mathcal{P}_{\mathcal{R}}^{\text {loop }}(k) \approx \frac{k^{3}}{4 \pi^{2}}\left(\frac{\lambda_{h}^{4}}{\pi^{2}} \frac{N(k)}{\epsilon}\right)\left(-i f\left(k, \tau, \tau_{\text {in }}\right)\right),
$$


where $f$ is an oscillatory function that includes all the oscillatory contributions arising from the external propagators, as given in eq. (5.22). The radiative corrections are compared to the tree-level contribution given by

$$
\mathcal{P}_{\mathcal{R}}^{\text {tree }}(k)=\frac{H^{2}}{8 \pi^{2} \epsilon M_{P}^{2}},
$$

giving a relative difference of

$$
\frac{\mathcal{P}_{\mathcal{R}}^{\text {loop }}(k)}{\mathcal{P}_{\mathcal{R}}^{\text {tree }}(k)}=\frac{2 \lambda_{h}^{4} N(k)}{\pi^{2}} \frac{M_{P}^{2}}{H^{2}}\left(-i k^{3} f\left(k, \tau, \tau_{\text {in }}\right)\right) .
$$

We define the quantity $C(k)$ in order to take into account all the time dependence of the amputated amplitude, i.e. the contribution of the external propagators plus the factor $a(\tau)^{4}$ of the vertex, then

$$
C(k)=\left|-i k^{3} f\left(k, \tau, \tau_{\text {in }}\right)\right|
$$

In Figure 7.2 we show the oscillatory behavior of $C(k)$. Note that for a very long inflationary phase, i.e. a large number of e-folds $N(k)$, the oscillations are suppressed and the factor $C(k)$ simplifies to a constant of order $\sim 0.2$.

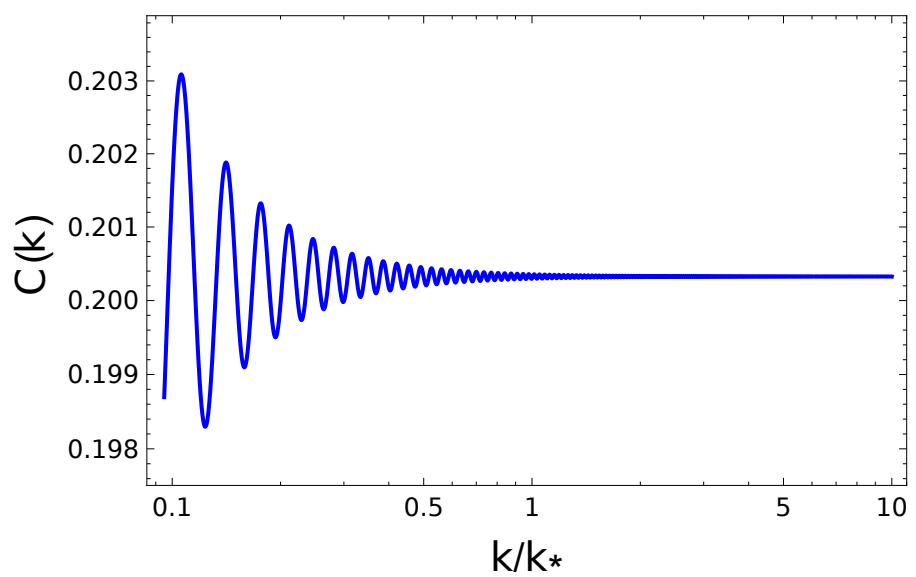

Figure 7.2: Oscillatory contribution arising from the external propagators $C(k)=$ $\left|-i k^{3} f\left(k,-1 / k, \tau_{\text {in }}\right)\right|$. The initial time is set to $\tau_{\text {in }}=-1 / k_{*} \exp \left(N_{\text {tot }}-N_{*}\right)$ with $N_{\text {tot }}=$ $62, k_{*}=0.05 \mathrm{Mpc}^{-1}$, and $N_{*}=57.5$.

In order to have a correction of $10^{-2}$ to the tree-level power spectrum, i.e.

$$
\left|\frac{\mathcal{P}_{\mathcal{R}}^{\text {loop }}(k)}{\mathcal{P}_{\mathcal{R}}^{\text {tree }}(k)}\right|=\frac{2 \lambda_{h}^{4} N(k)}{\pi^{2}} \frac{M_{P}^{2}}{H^{2}} C(k)<10^{-2},
$$


we need a coupling constant of

$$
\lambda_{h}^{2} \sim \frac{\pi}{10} \frac{H}{M_{P} \sqrt{2 N(k) C(k)}} .
$$

Moreover, the Hubble constant can be estimated in the slow-roll approximation from eq. (7.22)

$$
H^{2}=\frac{1}{3 M_{P}^{2}} \frac{m^{2} \phi^{2}}{2}=\frac{2}{3} m^{2} N(k) .
$$

In order to remain in the mass-dominated regime the coupling constant is constrained to be small. From eq. (7.28) one finds

$$
\lambda_{h}^{2}<2 \pi \frac{m}{2 M_{P} \sqrt{N(k)}}=\frac{\pi}{\sqrt{N(k)}} \frac{m}{M_{P}},
$$

and the maximal loop correction is estimated to be

$$
\begin{aligned}
\left|\frac{\mathcal{P}_{\mathcal{R}}^{\text {loop }}(k)}{\mathcal{P}_{\mathcal{R}}^{\text {tree }}(k)}\right| & \approx \frac{2 N(k)}{\pi^{2}} \frac{M_{P}^{2}}{H^{2}} C(k)\left(\frac{\lambda_{h}^{4}}{m^{2}}\right) m^{2} \\
& =\frac{2 N(k)}{\pi^{2}} \frac{M_{P}^{2}}{H^{2}} C(k)\left(\frac{\pi^{2}}{N(k)} \frac{1}{M_{P}^{2}}\right)\left(\frac{3 H^{2}}{2 N(k)}\right)<\frac{3 C(k)}{N(k)} \sim 10^{-2}
\end{aligned}
$$

We see that for the non-supersymmetric hybrid model, larger corrections cannot be reached because they are suppressed by the oscillatory function $C(k)$. Moreover, the e-folds number dependence $N(k)$ of the squared mass $(7.42)$ is compensated by the contribution from the coupling $\lambda_{h}$ giving an overall factor $1 / N(k)$. A larger number of e-folds would suppress both, the constant correction and the oscillations.

The power spectrum is shown in Figure 7.3 and was computed using the full massive propagators. The radiative corrections are amplified by a factor 50 in order to be visible in the plot. The oscillations appear when the contribution of the external propagators is included. As discussed in Chapter 6, the oscillations are a consequence of the initial state that was set to the Bunch-Davies vacuum at the beginning of inflation. The effect is much larger compared to the one estimated in the chaotic scenario.

This is expected for hybrid models since here the coupling is many order of magnitude larger with respect to monomial inflation. Moreover the correction here is proportional to the effective squared mass and we therefore expect contributions also 

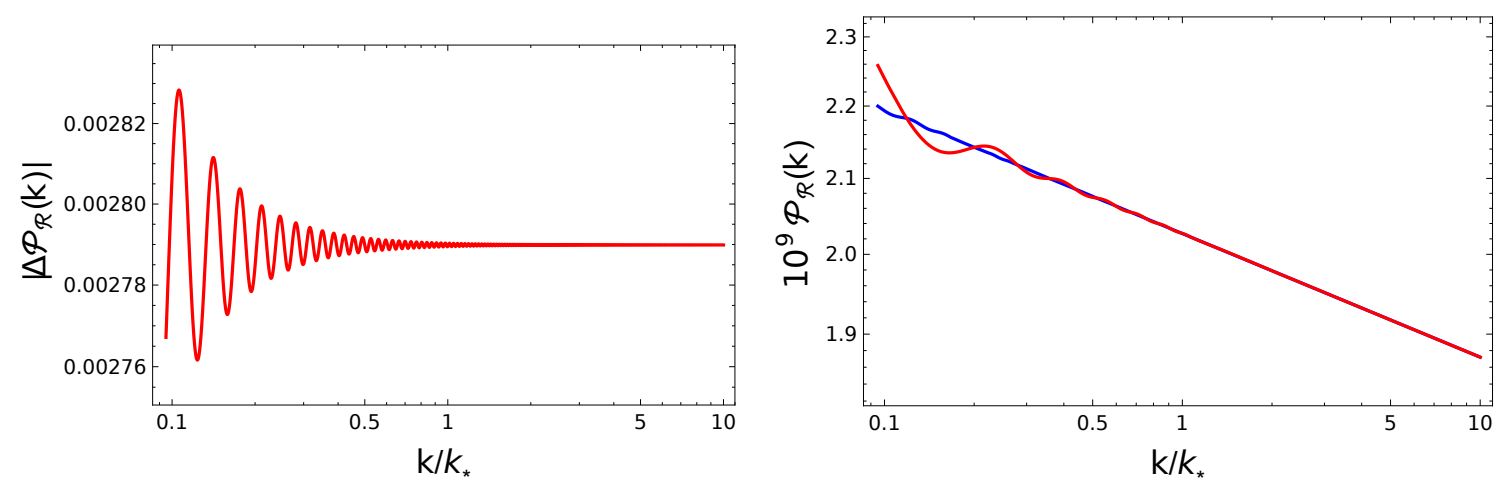

Figure 7.3: Relative difference and renormalized power spectrum for hybrid inflation obtained with the full propagator with $\lambda_{h}=\times 10^{-3}$ and $\mu$ of the order of $m_{\sigma, *} / 2$. The initial times are set to $\tau_{\text {in }}=-1 / k_{*} \exp \left(N_{\text {tot }}-N_{*}\right)$ with $N_{\text {tot }}=62, k_{*}=0.05 \mathrm{Mpc}^{-1}$ and $N_{*}=57.5$ (blue line) or $N_{*}=59$ (red line). In the right panel the corrections to the tree-level are amplified by a factor 50

from the heavy field. In the most optimistic case we found a relative difference of

$$
\left|\Delta \mathcal{P}_{\mathcal{R}}(k)\right|_{\text {hybrid }}=\left|\frac{\mathcal{P}_{\mathcal{R}}^{\text {loop }}(k)}{\mathcal{P}_{\mathcal{R}}^{\text {tree }}(k)}\right|_{\text {hybrid }} \leq 3 \times 10^{-3},
$$

which is still too small to be seen in the current observations and also in the future.

\section{Supersymmetric hybrid model}

In this subsection the radiative corrections are studied for the model that gave the most optimistic scenario, i.e. the hybrid model inspired by the supersymmetric theory with the scalar potential given in eq. (7.30), where the coupling determines the slope of the inflaton potential due to the one-loop Coleman-Weinberg potential. As pointed-out in the discussion of the classical dynamics in Section 7.1, here an inflationary phase is possible for values of $\lambda_{h}$ of the order of $10^{-3}$.

From eq. (7.30) the mass of the scalar component of the superfield $\Sigma$ and the Hubble scale can be esimated as

$$
m_{\Sigma}^{2}=2 \lambda_{h}^{2}\left(\phi^{2}-M_{G}^{2}\right), \quad 3 H^{2} \approx \frac{V}{M_{P}^{2}}=\lambda_{h}^{2} \frac{M_{G}^{4}}{M_{P}^{2}}
$$


Therefore we obtain

$$
\begin{aligned}
\frac{m_{\Sigma}^{2}}{H^{2}} & \approx 2 \lambda_{h}^{2}\left(\phi^{2}-M_{G}^{2}\right)\left(\frac{3 M_{P}^{2}}{\lambda_{h}^{2} M_{G}^{4}}\right) \\
& =\left(\frac{\lambda_{h}^{4} M_{P}^{2} N}{\pi^{2}}\right)\left(\frac{3 M_{P}^{2}}{\lambda_{h}^{2} M_{G}^{4}}\right) \\
& =\frac{3 \lambda_{h}^{2} N M_{P}^{4}}{\pi^{2} M_{G}^{4}} \sim 1.35 \times 10^{7} .
\end{aligned}
$$

The one-loop contribution is enhanced with respect to the quartic monomial inflation by a factor of $10^{7}$ due to the hybrid field's mass.

The power spectrum is shown in Figure 7.4. It was computed numerically with the full propagators with the Bunch-Davies vacuum set at the beginning of inflation. Moreover, because the massless de-Sitter counter-term in eq. (5.20) has a quadratic dependence on $H$ and the WKB counterterm in eq. (5.66) has a quadratic dependence on the mass, the effects of the former are negligible. The oscillatory features in the power spectrum emerge after the inclusion of the external propagators.
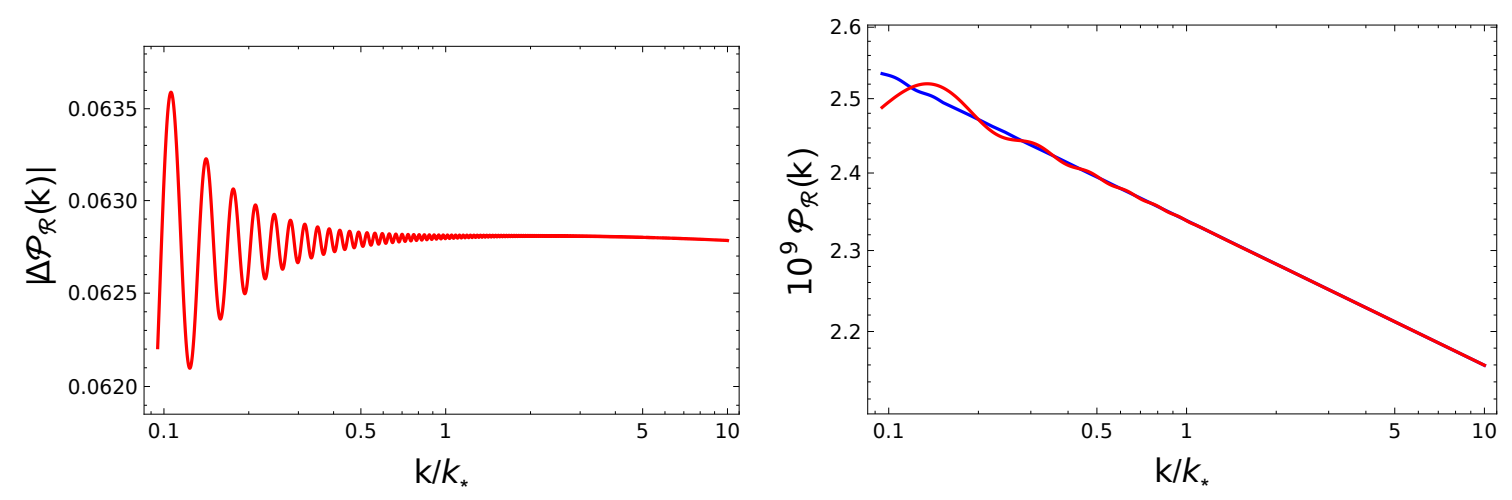

Figure 7.4: Relative difference and renormalized power spectrum for the hybrid supersymmetric model obtained with the full propagator with $\lambda_{h}=3 \times 10^{-3}$ and $\mu$ of the order of $3 \lambda_{h} M_{G}$. The initial times are set to $\tau_{\text {in }}=-1 / k_{*} \exp \left(N_{\text {tot }}-N_{*}\right)$ with $N_{\text {tot }}=62, k_{*}=0.05 \mathrm{Mpc}^{-1}$ and $N_{*}=57.5$ (blue line) or $N_{*}=59$ (red line).

In this case the radiative effects on the power spectrum are visible without any amplification. Indeed, the supersymmetric model has the largest coupling $\lambda_{h}$ that with the heaviness of the hybrid field gave us the most relevant scenario to see the periodic features in the primordial spectrum. In the most optimistic case we found a relative difference of

$$
\left|\Delta \mathcal{P}_{\mathcal{R}}(k)\right|_{\text {hybrid, } \mathrm{S}}=\left|\frac{\mathcal{P}_{\mathcal{R}}^{\text {loop }}(k)}{\mathcal{P}_{\mathcal{R}}^{\text {tree }}(k)}\right|_{\text {hybrid, } \mathrm{S}} \leq 0.7 \times 10^{-1},
$$

that is still too small to be detected with the current sensitivities of the today's instruments. 
An analytic estimate of the largest possible effect can be obtained from the spectrum normalization. For the most optimistic value of the coupling constant in eq. (7.36) the relative difference is estimated to be of order $\mathcal{O}(1)$

$$
\begin{aligned}
\left|\frac{\mathcal{P}_{\mathcal{R}}^{\text {loop }}(k)}{\mathcal{P}_{\mathcal{R}}^{\text {tree }}(k)}\right| & =\frac{\lambda_{h}^{2}}{4 \pi^{2}} \frac{m_{\Sigma}^{2}}{H^{2}} C(k)=\frac{1}{4 \pi^{2}}\left(\frac{3 N M_{P}^{4}}{\pi^{2} M_{G}^{4}}\right) \lambda_{h}^{4} C(k) \\
& <\frac{1}{4 \pi^{2}}\left(\frac{3 N M_{P}^{4}}{\pi^{2} M_{G}^{4}}\right)\left(16 \pi^{4} \frac{M_{G}^{4}}{100 M_{P}^{4}}\right) C(k) \\
& =0.12 N(k) C(k) \sim 1,
\end{aligned}
$$

giving an interesting range for large contributions. We remark that in this case the relative difference is proportional to the number of e-folds $N(k)$ because there is no compensation from the coupling $\lambda_{h}$. Therefore a larger number of e-folds would increase the constant effect but suppress more the oscillations as shown in Figure 7.2.

It should be noted that here we are considering only the loop contributions of the hybrid scalar field, while in the full supersymmetric model also the corresponding fermionic fields have to be considered [156] and they would give a negative contribution that will suppress the effect because of the cancellation of the quadratic divergence ${ }^{3}$. The fermion propagator in de-Sitter spacetime [157] is very similar to the scalar propagator given in terms of the hypergeometric function. We leave the explicit calculations for future works.

\section{Spectator field model}

To conclude our analysis about the radiative corrections to the primordial spectrum we present our results for the spectator field model. The renormalized amputated amplitude is given by eq. (5.67) where the mass $m$ is simply given by the spectator's mass $m_{\sigma}$. The one-loop corrections to the power spectrum are given by

$$
\mathcal{P}_{\mathcal{R}}^{\text {loop }}(k) \approx \frac{k^{3}}{4 \pi^{2}}\left(\frac{\lambda_{h}^{2} m_{\sigma}^{2}}{8 \pi^{2} \epsilon M_{P}^{2}}\right)\left(1+\log \left(\frac{m_{\sigma}^{2}}{4 \mu^{2}}\right)\right)\left(-i f\left(k, \tau, \tau_{\text {in }}\right)\right) .
$$

The classical dynamics was discussed in Section 7.1 where the largest effects are given for a spectator's mass of $m_{\sigma} \sim 10^{-2} M_{P}$ and the coupling constant $\lambda_{h}^{2} \sim 10^{-6}$. In these cases we estimated a maximal correction of

$$
\left|\frac{\mathcal{P}_{\mathcal{R}}^{\text {loop }}(k)}{\mathcal{P}_{\mathcal{R}}^{\text {tree }}(k)}\right|=\frac{3 \lambda_{h}^{2}}{8 \pi^{2}} \frac{m_{\sigma}^{2}}{m^{2}} \frac{C(k)}{N(k)}<0.4 \times 10^{-3},
$$

\footnotetext{
${ }^{3}$ The fermionic contribution is exactly canceling the quadratic divergence in the loop. A contribution proportional to difference between the scalar and fermion masses should survive, see [151].
} 
where $\mathcal{P}_{\mathcal{R}}^{\text {tree }}$ is given in (7.46) and the Hubble constant is estimated from (7.51). The expression is similar to the one for chaotic inflation with the enhancement due to the larger mass and coupling. On the other hand it is suppressed by the number of e-folds, giving a smaller contribution compared to the discussed hybrid models.

The power spectrum is shown in Figure 7.5 where the loop-correction was amplified by a factor 100 . The loop-correction was computed numerically with the full propagators as in the previous cases. The oscillatory features are smaller compared to the hybrid models as predicted in the estimate. With the discussed parameter choice we found a relative difference of

$$
\left|\Delta \mathcal{P}_{\mathcal{R}}(k)\right|_{\text {spect }}=\left|\frac{\mathcal{P}_{\mathcal{R}}^{\text {loop }}(k)}{\mathcal{P}_{\mathcal{R}}^{\text {tree }}(k)}\right|_{\text {spect }} \leq 4 \times 10^{-4}
$$
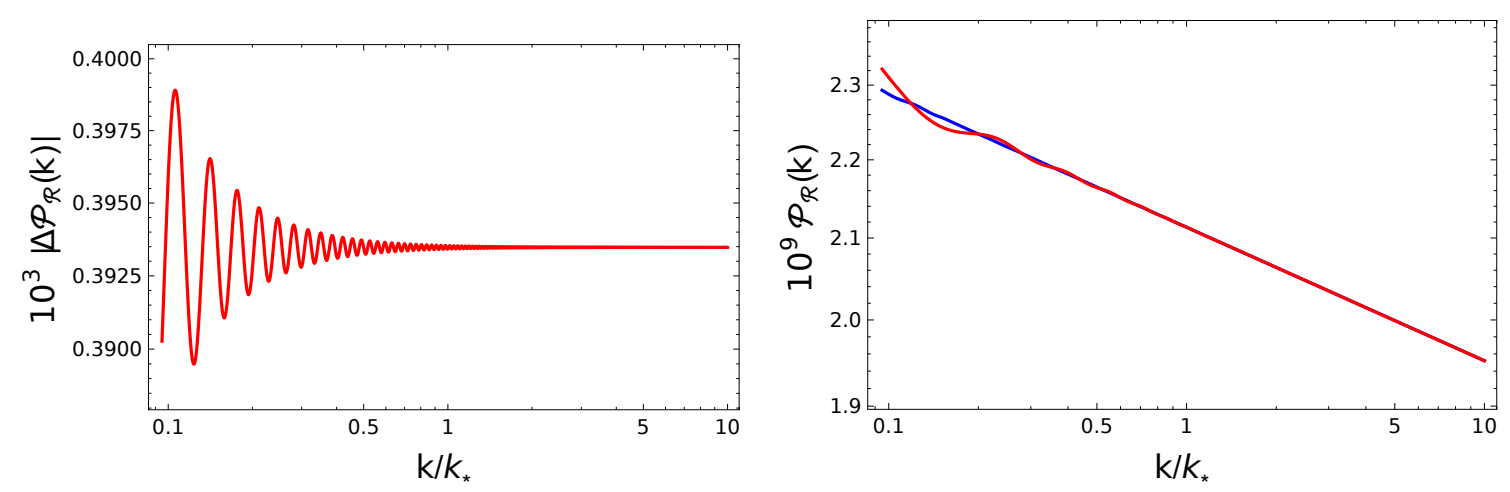

Figure 7.5: Relative difference and renormalized power spectrum for the spectator field model obtained with the full propagator with $\lambda_{h}=10^{-3}, m_{\sigma}=10^{-2} M_{P}$, and $\mu$ of the order of $m_{\sigma} / 2$. The initial times are set to $\tau_{\text {in }}=-1 / k_{*} \exp \left(N_{\text {tot }}-N_{*}\right)$ with $N_{\text {tot }}=62$, $k_{*}=0.05 \mathrm{Mpc}^{-1}$ and $N_{*}=57.5$ (blue line) or $N_{*}=59$ (red line). In the right panel the corrections to the tree-level are amplified by a factor $10^{2}$

In this section the physical impact of radiative corrections to the power spectrum was presented. In particular the massless and massive two-point correlation function in de-Sitter spacetime derived in Section 5.1 and 5.3 are applied to simple models as the chaotic scenario and the hybrid models introduced in Section 7.1. Because the one-loop corrections are proportional to $\lambda$, the effects in the chaotic scenario of a monomial quartic interaction are suppressed by a factor of the order of $10^{-13}$. In the case of hybrid inflation the largest effect is given by the supersymmetric model given by the potential (7.29) where the corrections are of the order of $\sim 0.1$. Unfortunately, with the current instrument sensitivities the effects are not enough to be detected. In all examples the loop-corrections gave a tiny shift in normalization that can be reabsorbed in the definition of $\lambda$. The inclusion of the external propagators is responsible for the 
features in the power spectrum. Their origin was discussed in Chapter 6. Note that they had no impact in the overall normalization and on the spectral index.

\subsection{The non-linearity parameter $\tau_{\mathrm{NL}}$}

This section is devoted to the discussion of the physical consequences of loop corrections to the trispectrum in the Schwinger and Keldysh formalism. We will present our prediction of the cosmological parameter $\tau_{\mathrm{NL}}$ that is a good indicator for potential non-Gaussian features in the primordial spectrum. The $\tau_{\mathrm{NL}}$ parameter is of particular interest because it can be estimated from CMB measurements [158]. Unfortunately the actual constraints are very weak and there is no clear indication of periodic features in the trispectrum. On the other hand they are not excluded.

The connected trispectrum was defined in eq. (5.25) and is the first non-zero higher order correlation function on a scalar field theory with quartic interaction term. The bispectrum and all the higher odd correlation functions are identically zero at first order because our system is invariant under the symmetry $\phi \rightarrow-\phi$. A model with precise Gaussianity predicts $\mathcal{T}_{\mathcal{R}}=0$ at tree-level. Departures from exact Gaussianity can be described by introducing the dimensionless cosmological parameter $\tau_{\mathrm{NL}}$ [42]

$$
\mathcal{T}_{\mathcal{R}}\left(\vec{k}_{1}, \vec{k}_{2}, \vec{k}_{3}, \vec{k}_{4}\right)=\frac{1}{2} \tau_{\mathrm{NL}}\left[\left(\frac{2 \pi^{2}}{k_{1} k_{2} k_{14}}\right)^{3} \mathcal{P}_{\mathcal{R}}\left(k_{1}\right) \mathcal{P}_{\mathcal{R}}\left(k_{2}\right) \mathcal{P}_{\mathcal{R}}\left(k_{14}\right)+23 \text { permutations }\right],
$$

where $\vec{k}_{i j}=\vec{k}_{i}+\vec{k}_{j}$ and $\sum_{i} \vec{k}_{i}=0$.

The non-linearity parameter $\tau_{\mathrm{NL}}$ is an observable quantity that depends on the inflationary model. Therefore it could be used to disentangling among different inflationary scenarios. From eqs. (5.25) and (7.62) we have

$$
\tau_{\mathrm{NL}}=\frac{1}{2 \epsilon^{2} M_{P}^{4}} \frac{1}{\left(2 \pi^{2}\right)^{3}} \frac{\left\langle\delta \phi_{k_{1}} \delta \phi_{k_{2}} \delta \phi_{k_{3}} \delta \phi_{k_{4}}\right\rangle_{c}^{\prime}}{\frac{\mathcal{P}_{\mathcal{R}}\left(k_{1}\right) \mathcal{P}_{\mathcal{R}}\left(k_{2}\right) \mathcal{P}_{\mathcal{R}}\left(k_{14}\right)}{k_{1}^{3} k_{2}^{3} k_{14}^{3}}+23 \text { permutations }},
$$

where the prime means that the overall $\delta$-function in eq. (5.25) is factorized out. All the external momenta $k_{i}$ are on super-Hubble scales, i.e. $\min \left(k_{i}\right) \tau=-1$.

In Section 5.2 we analyzed the time dependence of the tree-level contribution and of the radiative one-loop corrections to the four-point correlation function. In Figure 5.9 and in eq. (5.29) we presented the tree-level contribution for a massive scalar field with quartic interaction term. The one-loop corrections are shown in Figure 5.7. Unfortunately they are suppressed by the overall factor $\lambda^{2}$ and they can be neglected when compared to the tree-level result. 
In the following we will consider the effects of the tree-level contribution to the four-point function on $\tau_{\mathrm{NL}}$ for an equilateral configuration ${ }^{4}$. As already discussed the oscillatory features arise because we set the Bunch-Davies vacuum state at the beginning of inflation. We expect to see the imprint of these spectral effects also on the non-linearity parameter $\tau_{\mathrm{NL}}$.

In Figure 7.6 the non-linearity parameter is plotted for the equilateral configuration for the maximal case of $\cos \theta_{1 i}=-1 / 3$ for all $i$. We found a function that is strongly oscillating around the value of $4 \times 10^{-7}$. This value is too small to be observed in the current Planck observations that measure a value of $g_{\mathrm{NL}}^{\text {local }}=(-9 \pm 7.7) \times 10^{4}$, where $\tau_{\mathrm{NL}} \sim \frac{18}{25} g_{\mathrm{NL}}^{\text {local }}$. Because of the weak constraints on $\tau_{\mathrm{NL}}$ due to the large error bars, the present observations do not exclude our prediction.

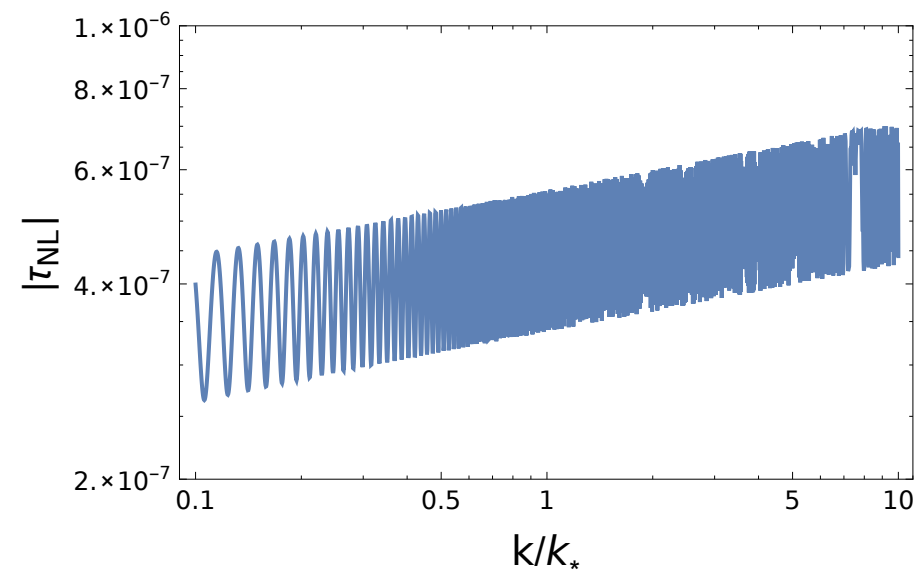

Figure 7.6: Non-linearity parameter $\left|\tau_{\mathrm{NL}}\right|$ calculated from the contribution $T_{1}+T_{2}$ for a $\lambda \phi^{4}$-theory with $\lambda=2.97 \times 10^{-13}$. The initial time is set to $\tau_{\text {in }}=-1 / k_{*} \exp \left(N_{\text {tot }}-N_{*}\right)$ with $N_{\text {tot }}=62$ and $N_{*}=57.5$.

\section{Massive one-loop}

In Section 5.2 we did not calculate the perturbative contributions to the four-point functions from massive fields in de-Sitter spacetime. The main reason is that the numerical calculations involving the hypergeometric function were already very involved for the renormalization of the two-point function where only one momentum integral had to be performed. In principle one could use the WKB propagator (4.46) as for the massive two-point function. However, in this case there are two distinct virtual times for the internal propagators and the full WKB propagator has to be used, making the calculations more difficult. The finite time effects of the tree-level and one-loop

\footnotetext{
${ }^{4}$ By denoting $\theta_{1 i}$ the angle between $\vec{k}_{1}$ and $\vec{k}_{i}$, the maximal effect is obtained for $\cos \theta_{1 i}=-1 / 3$ for all $i[42]$.
} 
radiative corrections from a massive field for a de-Sitter background is left for a future work. Before concluding, we give a rough estimate of the massive contribution to the four-point function.

In the case of hybrid inflation we do not have a tree-level contribution because of the absence of the self-coupling in the theory that we neglected in our hybrid models. Therefore the first dominant contribution in the massive case comes from the one-loop perturbative correction. For dimensional reasons we expect that the first order radiative corrections contain only terms proportional to $m_{\Sigma}^{4}, m_{\Sigma}^{2} H^{2}$, and $H^{2}$. Since $H^{2}$ is suppressed compared to $m_{\Sigma}^{2}$, the maximal contribution will be proportional to $m_{\Sigma}^{4}$. The ratio between the hybrid and the chaotic model contribution is estimated to be

$$
\frac{\left[A_{4, \mathrm{amp}}\right]^{\text {hybrid }}}{\left[A_{4, \mathrm{amp}}\right]^{\text {chaotic }}} \propto \frac{\lambda_{h}^{4} m_{\Sigma}^{4}}{\lambda H^{4}}=7.1 \times 10^{8} .
$$

In our naive estimation $\tau_{\mathrm{NL}}$ may become of order 1 , but it is still not big enough to be observed.

For the case of a spectator field that is not influencing the inflaton classical dynamics, with $m_{\sigma} \sim 10^{-2} M_{P}$ and $\lambda_{h}^{2} \sim 10^{-6}$, we estimated a larger contribution

$$
\frac{\left[A_{4, \mathrm{amp}}\right]^{\text {hybrid }}}{\left[A_{4, \mathrm{amp}}\right]^{\text {chaotic }}} \propto \frac{\lambda_{h}^{4} m_{\sigma}^{4}}{\lambda H^{4}}=2.3 \times 10^{11},
$$

which may become relevant if it is not canceled by the other terms that appear in the full calculation.

The results presented here show the physical impact of the finite time contributions to the non-linearity parameter $\tau_{\mathrm{NL}}$ in the case of a scalar field theory with a quartic self-interaction term. The oscillatory features that we predict for the four-point function are imprinted also in the trispectrum and are shown in Figure 7.6. The non-linearity parameter strongly oscillates about the value of $4 \times 10^{-7}$, which is too small to be observed with current constraints. We also neglected the one-loop corrections because they are suppressed by a factor $\lambda^{2}$. Our rough estimates for hybrid models have shown that in the case of an inflationary scenario with a spectator field the contribution may reach an interesting regime where the effect can be large enough to be observed. The full calculation in the case of hybrid inflation is left for future works. 


\section{CHAPTER 8}

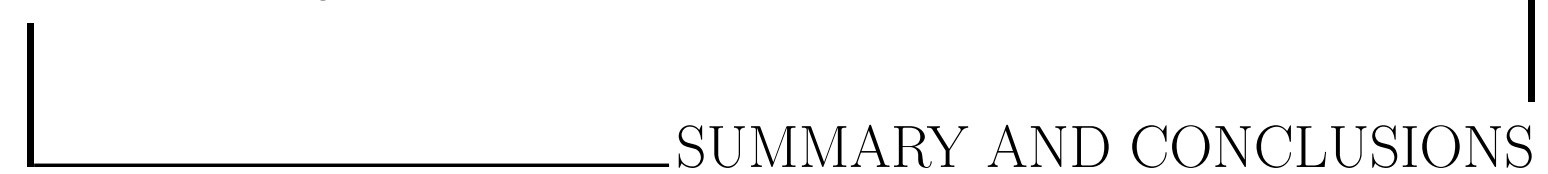

Cosmic inflation is one of the most successful and fundamental theories in cosmology. It describes a period of accelerating expansion of the very primordial universe. This theory provides the most natural scenario to solve the main issues of the big bang theory. The inflationary paradigm provides the initial conditions required by the big bang expansion to explain current cosmological observations.

In the standard scenario, the quantum fluctuations of the inflaton field $\delta \phi$ generate the primordial fluctuations of the curvature tensor and are responsible for the temperature anisotropies in the cosmic microwave background and the origin of the large scale structure of the universe.

The spectrum of the primordial perturbations is predicted to be nearly scale invariant due to the slow-rolling of the inflaton field. The power-law behavior is well-fitted with the latest Planck data [6] where they found no evidence for any particular deviation. The primordial spectrum provides the point of connection between observations and inflationary scenarios because of its model dependence.

The classical dynamics of various inflationary scenarios [7] have already been investigated for many models and the resulting cosmological parameters are in good agreement with observations. Less attention has been devoted to the time dependence of the intrinsic quantum corrections arising both due to the background evolution and from the initial Bunch-Davies vacuum state. This gives a time dependence that leaves an imprint on observable quantities like the power spectrum as well as higher order spectra.

In this thesis the renormalization procedure in curved spacetime was studied for the simple example of a scalar field model with a quartic self-interaction term in the closedtime-path formalism for a (quasi) de-Sitter universe. The model was subsequently 
extended to the scenario of two scalar fields with a quartic symmetric coupling. In both cases, quantum corrections generate time-dependent oscillations in the two- and four-point correlation function. The appearance of the spectral features was analyzed in Minkowski spacetime, where it is expected that the non-local time dependence is due to the initial time and was investigated by testing the renormalized physical observables with different continuous switching-on interaction profiles. The issue about the adiabatic limit was also discussed.

Two- and four-point correlation functions were renormalized in the minimal subtraction scheme. The counter-terms found in de-Sitter background are independent from the geometry of the spacetime, as proven in the algebraic QFT framework. The counter-terms are in agreement with similar results using dimensional regularization [33], the effective action method [34], or in axiomatic field theory [37, 38]. In the adiabatic subtraction scheme the analysis of [34] was extended to the case where the renormalization conditions are given in de-Sitter spacetime, giving extra covariant contributions to the finite part of the counter-terms.

In the FRW universe, the background generates time-dependent terms proportional to the logarithm of the Hubble parameter. The oscillations were found to be a consequence of the initial conditions. The one-loop corrections to the two-point function are suppressed by the quartic coupling and enhanced by the infrared cutoff. Here, we conservatively used an IR-cutoff of the order of the perturbation's effective mass $\propto\left|n_{s}-1\right|$. Other prescriptions [154] used the Hubble scale at the beginning of inflation as an infrared cutoff, and predicted an effect that depends on the duration of the inflationary phase. In this case the constant contribution of the radiative corrections becomes dominant and the early oscillations are suppressed. The same considerations are valid for the four-point function where the loop-corrections are suppressed by the squared value of the coupling. In this case the tree-level contributions also depend on the initial state and generate non-local contributions. These oscillations are in general damped during the inflationary epoch and therefore they affect strongly the large scales (since they leave the horizon earlier). The calculated correlation functions were finally used in order to test the quantum nature of the inflaton field and to predict the radiative corrections to observable quantities, such as the power spectrum and the non-linearity parameter that indicates a deviation from Gaussianity.

In the second part of the thesis the effects of one-loop corrections were predicted for simple inflationary models. For the chaotic scenario of the $\lambda \phi^{4}$ model the one-loop correction is suppressed by the coupling constant that is fixed to be of the order of $10^{-13}$ by the normalization of the primordial spectrum. The effect is very small and a relative difference of $\left|\Delta \mathcal{P}_{\mathcal{R}}(k)\right| \leq 10^{-13}$ was found. This result strongly depends on the 
IR cutoff and much larger values can be reached with other prescriptions $[154,155]$, which however have the disadvantage of suppressing the oscillations.

The second class of models that was investigated consisted of a two scalar field model where the inflaton gets radiative corrections from virtual effects of the heavier field. In this hybrid scenario, the field in the loop is very massive and the coupling can take values up to order $\lambda_{h} \sim 10^{-3}$. The contribution to the power spectrum was computed for the first time with the full massive propagator given in terms of the hypergeometric function and the counter-terms were computed analytically in the WKB regime. The larger coupling and the presence of the heavier field increased the size of the radiative correction up to a relative difference of $\left|\Delta \mathcal{P}_{\mathcal{R}}(k)\right| \leq 3 \times 10^{-3}$. In order to enhance even more the one-loop contribution, the supersymmetric hybrid model [151] was discussed and gave a relative difference of $\left|\Delta \mathcal{P}_{\mathcal{R}}(k)\right| \leq 10^{-1}$ in the most optimistic scenario. It should be noted that in a supersymmetric setup, the quadratic divergence is canceled by the fermionic contribution. The full calculation with the inclusion of the fermion contribution is of particular interest and is a suggested direction for future works. The model of a quadratic inflation with a spectator field was also investigated and gave weaker bounds. Unfortunately the oscillations, even if they are non-negligible for the hybrid model, are too small to be observed or excluded by the present data.

Finally, the one-loop corrections to the trispectrum were computed by focusing on the effects on the non-linearity parameter. In the chaotic scenario, oscillations were found with an amplitude around the value of $\left|\tau_{\mathrm{NL}}\right| \sim 4 \times 10^{-7}$, which is too suppressed to be observed. It was estimated that it could become relevant for hybrid models or for spectator fields. The explicit calculations for the massive contributions is left for a future work.

The latest Planck data seem to prefer a power-law primordial spectrum without any particular feature [6]. Nevertheless, a departure from scale invariance is allowed due to the weak constraints on featured power spectra. In the future, progress in the reconstruction of the primordial universe are expected and stronger constraints on the power spectrum and higher order spectra will give the possibility to see the imprint of the quantum nature of the inflaton field for models with a sufficiently large coupling, as in the hybrid scenario. 


\section{APPENDIX A}

FEYNMAN RULES

In this appendix the Feynman rules for the two main models considered in this thesis are described, i.e. a single scalar field model with quartic self-interaction and a two-scalar fields model with a quartic symmetric coupling.

\section{A.1 $\lambda \phi^{4}$-theory}

The action for the $\lambda \phi^{4}$-interacting field theory of eq. (7.1) is, in the closed-time-path formalism with a classical background $g_{\mu \nu}$, given by

$$
S\left[\phi^{+}, \phi^{-}\right]=\int_{\tau_{\text {in }}}^{\infty} d \tau \int d^{3} x \mathcal{L}\left[\phi^{+}, \phi^{-}\right]
$$

where $\tau_{\text {in }}$ is the initial time where we assumed the system to be in a vacuum state, $\phi^{+}$ and $\phi^{-}$are the two components of the field according to the CTP notation, and

$$
\begin{aligned}
\mathcal{L}\left[\phi^{+}, \phi^{-}\right]=\sqrt{-g} & {\left[\frac{1}{2} \partial_{\mu} \phi^{+} \partial^{\mu} \phi^{+}-\frac{1}{2} m^{2}\left(\phi^{+}\right)^{2}-\frac{\lambda}{4 !}\left(\phi^{+}\right)^{4}+\frac{\xi}{2} \mathcal{R}\left(\phi^{+}\right)^{2}\right.} \\
& \left.-\frac{1}{2} \partial_{\mu} \phi^{-} \partial^{\mu} \phi^{-}+\frac{1}{2} m^{2}\left(\phi^{-}\right)^{2}+\frac{\lambda}{4 !}\left(\phi^{-}\right)^{4}-\frac{\xi}{2} \mathcal{R}\left(\phi^{-}\right)^{2}\right]+\delta \mathcal{L} .
\end{aligned}
$$

In the previous expression the metric $g_{\mu \nu}$ has signature,$+--- g=\operatorname{det}\left(g_{\mu \nu}\right)$ and the counter-terms are defined as

$$
\delta \mathcal{L}=\sqrt{-g}\left(\frac{1}{2} \delta Z \partial_{\mu} \phi^{+} \partial^{\mu} \phi^{+}-\frac{1}{2} \delta m^{2}\left(\phi^{+}\right)^{2}-\frac{\delta \lambda}{4 !}\left(\phi^{+}\right)^{4}+\frac{\delta \xi}{2} \mathcal{R}\left(\phi^{+}\right)^{2}\right)-\phi^{+} \leftrightarrow \phi^{-} .
$$


In the Schwinger basis (4.17) the Lagrangian reads

$$
\begin{aligned}
\mathcal{L}\left[\phi^{(1)}, \phi^{(2)}\right]=\sqrt{-g}\left(\partial_{\mu} \phi^{(1)} \partial^{\mu} \phi^{(2)}-m^{2} \phi^{(1)} \phi^{(2)}-\frac{\lambda}{4 !} \phi^{(1)}\left(\phi^{(2)}\right)^{3}\right. & \\
& \left.-\frac{\lambda}{3 !}\left(\phi^{(1)}\right)^{3} \phi^{(2)}+\frac{\xi}{3} \mathcal{R} \phi^{(1)} \phi^{(2)}\right)+\delta \mathcal{L} .
\end{aligned}
$$

From this Lagrangian one can easily extract the CTP Feynman rules needed in our perturbative analysis. In Table A.1 we list the rules for the two-point functions, the self-interacting vertices and the counter-terms for $\xi=0$. We represent $\phi^{(1)}$ with a solid line and $\phi^{(2)}$ with a dotted line.

Table A.1: Feynman rules for the $\lambda \phi^{4}$ theory. The scalar field $\phi^{(1)}$ is represented by a

\begin{tabular}{|c|c|}
\hline Graph & Expression \\
\hline & $F\left(k, \tau_{1}, \tau_{2}\right)$ \\
\hline & $-i G^{R}\left(k, \tau_{1}, \tau_{2}\right)=-i G^{A}\left(k, \tau_{2}, \tau_{1}\right)$ \\
\hline & $-i a^{4}\left(\tau_{1}\right) \frac{\lambda}{4 !} \delta\left(\tau_{1}-\tau_{2}\right) \delta\left(\tau_{1}-\tau_{3}\right) \delta\left(\tau_{1}-\tau_{4}\right)$ \\
\hline & $-i a^{4}\left(\tau_{1}\right) \frac{\lambda}{3 !} \delta\left(\tau_{1}-\tau_{2}\right) \delta\left(\tau_{1}-\tau_{3}\right) \delta\left(\tau_{1}-\tau_{4}\right)$ \\
\hline & $-i a^{4}\left(\tau_{1}\right) \delta m^{2} \delta\left(\tau_{1}-\tau_{2}\right)$ \\
\hline & $-i a^{4}\left(\tau_{1}\right) \frac{\delta \lambda}{4 !} \delta\left(\tau_{1}-\tau_{2}\right) \delta\left(\tau_{1}-\tau_{3}\right) \delta\left(\tau_{1}-\tau_{4}\right)$ \\
\hline & $-i a^{4}\left(\tau_{1}\right) \frac{\delta \lambda}{3 !} \delta\left(\tau_{1}-\tau_{2}\right) \delta\left(\tau_{1}-\tau_{3}\right) \delta\left(\tau_{1}-\tau_{4}\right)$ \\
\hline
\end{tabular}
solid line and the field $\phi^{(2)}$ by a dotted line. 


\section{A.2 Hybrid model}

The in-in action for a two-scalar field theory with a quartic interaction term $\lambda_{h}^{2} \phi^{2} \sigma^{2}$ reads

$$
S\left[\phi^{+}, \phi^{-}, \sigma^{+}, \sigma^{-}\right]=\int_{\tau_{\text {in }}}^{\infty} d \tau \int d^{3} x \mathcal{L}\left[\phi^{+}, \phi^{-}, \sigma^{+}, \sigma^{-}\right],
$$

where also the second field was split in the two components $\sigma^{+}$and $\sigma^{-}$. The Lagrangian density is given by

$$
\begin{gathered}
\mathcal{L}\left[\phi^{+}, \phi^{-}, \sigma^{+}, \sigma^{-}\right]=\sqrt{-g}\left[\frac{1}{2} \partial_{\mu} \phi^{+} \partial^{\mu} \phi^{+}+\frac{1}{2} \partial_{\mu} \sigma^{+} \partial^{\mu} \sigma^{+}-\frac{1}{2} m^{2}\left(\phi^{+}\right)^{2}-\frac{1}{2} M^{2}\left(\sigma^{+}\right)^{2}-\lambda_{h}^{2}\left(\phi^{+}\right)^{2}\left(\sigma^{+}\right)^{2}\right. \\
\left.-\frac{1}{2} \partial_{\mu} \phi^{-} \partial^{\mu} \phi^{-}-\frac{1}{2} \partial_{\mu} \sigma^{-} \partial^{\mu} \sigma^{-}+\frac{1}{2} m^{2}\left(\phi^{-}\right)^{2}+\frac{1}{2} M^{2}\left(\sigma^{-}\right)^{2}+\lambda_{h}^{2}\left(\phi^{-}\right)^{2}\left(\sigma^{-}\right)^{2}\right]+\delta \mathcal{L}
\end{gathered}
$$

where the counter-terms are defined as

$$
\begin{aligned}
\delta \mathcal{L} & =\sqrt{-g}\left(\frac{1}{2} \delta Z_{\phi} \partial_{\mu} \phi^{+} \partial^{\mu} \phi^{+}+\frac{1}{2} \delta Z_{\sigma} \partial_{\mu} \sigma^{+} \partial^{\mu} \sigma^{+}\right. \\
& \left.-\frac{1}{2} \delta m^{2}\left(\phi^{+}\right)^{2}-\frac{1}{2} \delta M^{2}\left(\sigma^{+}\right)^{2}-\delta \lambda_{h}^{2}\left(\phi^{+}\right)^{2}\left(\sigma^{+}\right)^{2}\right)-\left\{\phi^{+}, \sigma^{+}\right\} \leftrightarrow\left\{\phi^{-}, \sigma^{-}\right\}
\end{aligned}
$$

In the Schwinger basis (4.17) the Lagrangian reads

$$
\begin{gathered}
\mathcal{L}\left[\phi^{(1)}, \phi^{(2)}, \sigma^{(1)}, \sigma^{(2)}\right]=\sqrt{-g}\left(\partial_{\mu} \phi^{(1)} \partial^{\mu} \phi^{(2)}+\partial_{\mu} \sigma^{(1)} \partial^{\mu} \sigma^{(2)}-m^{2} \phi^{(1)} \phi^{(2)}-M^{2} \sigma^{(1)} \sigma^{(2)}\right. \\
\left.-2 \lambda_{h}^{2} \phi^{(1)} \phi^{(2)}\left(\sigma^{(1)}\right)^{2}-2 \lambda_{h}^{2}\left(\phi^{(1)}\right)^{2} \sigma^{(1)} \sigma^{(2)}-\frac{\lambda_{h}^{2}}{2}\left(\phi^{(2)}\right)^{2} \sigma^{(1)} \sigma^{(2)}-\frac{\lambda_{h}^{2}}{2} \phi^{(1)} \phi^{(2)}\left(\sigma^{(2)}\right)^{2}\right)+\delta \mathcal{L} .
\end{gathered}
$$

From the Lagrangian, the closed-time-path Feynman rules can be identified. They are listed in Table A.2 for the two-point functions, the self-interacting vertices and the counter-terms. The field $\phi^{(1)}$ is represented by a solid single line, $\phi^{(2)}$ by a dotted single line, $\sigma^{(1)}$ by a solid double line, and $\sigma^{(2)}$ by a dotted double line. 
Table A.2: Feynman rules for the two scalar field theory (A.8). In the expressions we omitted the Dirac delta functions that can be reconstructed from Table A.1. The light fields $\phi^{(1)}$ and $\phi^{(2)}$ are represented by a solid and dotted single line, the heavy fields $\sigma^{(1)}$ and $\sigma^{(2)}$ by a solid and dotted double line.

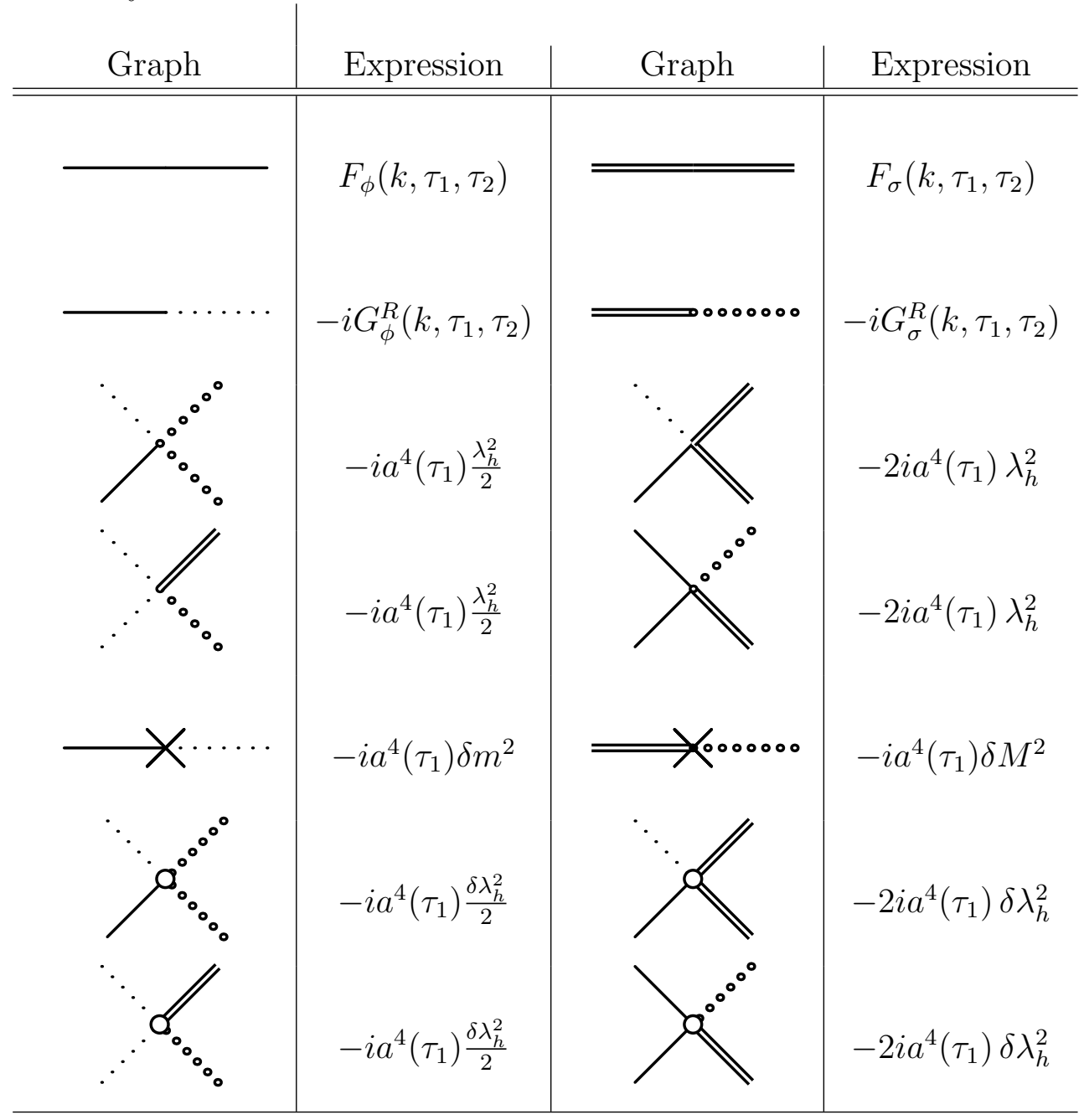




\section{APPENDIX B}

USEFUL INTEGRALS

In this appendix we list the integrals ${ }^{1}$ needed in Section 5.2 for the one-loop corrections to the four-point function in de-Sitter spacetime.

$$
\begin{aligned}
& I_{1}=\int_{\tau_{\text {in }}}^{\tau} d \tau_{1} \int_{\tau_{\text {in }}}^{\tau} d \tau_{2} \frac{\left(\tau^{3}-\tau_{1}^{3}\right)\left(\tau^{3}-\tau_{2}^{3}\right)}{\tau_{1}^{4} \tau_{2}^{4}}=\frac{1}{9}\left(3 \log \left(\frac{\tau}{\tau_{\text {in }}}\right)+1\right)^{2}+\mathcal{O}\left(\frac{\tau}{\tau_{\text {in }}}\right), \\
& I_{2}=\int_{\tau_{\text {in }}}^{\tau} d \tau_{1} \int_{\tau_{\text {in }}}^{\tau} d \tau_{2} \frac{\left(\tau^{3}-\tau_{1}^{3}\right)\left(\tau^{3}-\tau_{2}^{3}\right) \log \left(k^{2} \tau_{1} \tau_{2}\right)}{\tau_{1}^{4} \tau_{2}^{4}} \\
& =\frac{1}{27}\left(3 \log \left(\frac{\tau}{\tau_{\text {in }}}\right)+1\right)\left(9 \log \left(\frac{\tau}{\tau_{\text {in }}}\right) \log \left(k^{2} \tau \tau_{\text {in }}\right)+6 \log (-k \tau)+2\right)+\mathcal{O}\left(\frac{\tau}{\tau_{\text {in }}}\right),
\end{aligned}
$$

$$
I_{3}=\int_{\tau_{\text {in }}}^{\tau} d \tau_{1} \int_{\tau_{\text {in }}}^{\tau_{1}} d \tau_{2} \frac{\left(\tau^{3}-\tau_{1}^{3}\right)\left(\tau_{1}^{3}-\tau_{2}^{3}\right)}{\tau_{1}^{4} \tau_{2}^{4}}=\frac{1}{6}\left(2+\log \left(\frac{\tau}{\tau_{\text {in }}}\right)\left(3 \log \left(\frac{\tau}{\tau_{\text {in }}}\right)+4\right)\right),
$$

$$
I_{4}=\int_{\tau_{\text {in }}}^{\tau} d \tau_{1} \int_{\tau_{\text {in }}}^{\tau_{1}} d \tau_{2} \frac{\left(\tau^{3}-\tau_{1}^{3}\right)\left(\tau_{1}-\tau_{2}\right)}{\tau_{1}^{3} \tau_{2}^{3}}=\frac{1}{12}\left(-11-6 \log \left(\frac{\tau}{\tau_{\text {in }}}\right)\right)
$$

\footnotetext{
${ }^{1}$ The integrals are evaluated with the analytic methods of Mathematica [139].
} 


$$
\begin{array}{r}
I_{5}=\int_{\tau_{\text {in }}}^{\tau} d \tau_{1} \int_{\tau_{\text {in }}}^{\tau_{1}} d \tau_{2} \frac{\left(\tau^{3}-\tau_{1}^{3}\right)\left(-\tau_{2}^{3} \log \left(\left|\frac{\tau_{1}}{2\left(\tau_{1}-\tau_{2}\right)}\right|\right)+\tau_{1}^{3} \log \left(\left|\frac{\tau_{2}}{2\left(\tau_{1}-\tau_{2}\right)}\right|\right)\right)}{\tau_{1}^{4} \tau_{2}^{4}} \\
=\frac{1}{108}\left\{6 \log \left(\frac{\tau}{\tau_{\text {in }}}\right)\left[3 \log \left(\frac{\tau}{\tau_{\text {in }}}\right)\left(\log \left(\frac{\tau}{8 \tau_{\text {in }}}\right)+1\right)-3 \pi^{2}+13-12 \log (2)\right]\right. \\
\left.-108 \zeta(3)-12 \pi^{2}+97-36 \log (2)\right\},
\end{array}
$$

$$
\begin{aligned}
& I_{6}=\int_{\tau_{\mathrm{in}}}^{\tau} d \tau_{1} \int_{\tau_{\mathrm{in}}}^{\tau_{1}} d \tau_{2} \frac{\left(\tau^{3}-\tau_{1}^{3}\right)\left(\log \left(-\frac{2 \eta \mu}{H \tau_{1}}\right)+\gamma\right)}{\tau_{1}^{4}} \delta\left(\tau_{1}-\tau_{2}\right)+\frac{\left(\tau^{3}-\tau_{1}^{3}\right)}{\tau_{1}^{2} \tau_{2}^{2}\left(\tau_{1}-\tau_{2}\right)} \theta\left(\tau_{1}-\tau_{2}-\eta\right) \\
= & \frac{1}{6}\left[-6\left(\log \left(\frac{2 \mu}{H}\right)+\gamma-1\right) \log \left(\frac{\tau}{\tau_{\text {in }}}\right)-2 \log \left(\frac{\mu}{H}\right)-\pi^{2}-2 \gamma+8-\log (4)\right]+\mathcal{O}\left(\frac{\tau}{\tau_{\text {in }}}\right),
\end{aligned}
$$

$$
I_{7}=\int_{\tau_{\mathrm{in}}}^{\tau} d \tau_{1} \int_{\tau_{\mathrm{in}}}^{\tau_{1}} d \tau_{2} \frac{\left(\tau^{3}-\tau_{1}^{3}\right)\left(\tau^{3}-\tau_{2}^{3}\right)\left(\tau_{1}^{3}-\tau_{2}^{3}\right)}{\tau_{1}^{4} \tau_{2}^{4}}=-\frac{1}{36} \tau_{\text {in }}^{6}\left(6 \log \left(\frac{\tau}{\tau_{\text {in }}}\right)+5\right)
$$

$$
I_{8}=\int_{\tau_{\mathrm{in}}}^{\tau} d \tau_{1} \int_{\tau_{\mathrm{in}}}^{\tau_{1}} d \tau_{2} \frac{\left(\tau^{3}-\tau_{1}^{3}\right)\left(\tau^{3}-\tau_{2}^{3}\right)^{2}\left(\tau_{1}-\tau_{2}\right)}{\tau_{1}^{3} \tau_{2}^{3}}=\frac{\tau_{\mathrm{in}}^{6}}{12}+\mathcal{O}\left(\frac{\tau}{\tau_{\mathrm{in}}}\right)
$$

$$
\begin{aligned}
I_{9} & =\int_{\tau_{\text {in }}}^{\tau} d \tau_{1} \int_{\tau_{\text {in }}}^{\tau_{1}} d \tau_{2} \frac{\left(\tau^{3}-\tau_{1}^{3}\right)\left(\tau^{3}-\tau_{2}^{3}\right)^{2}\left(\tau_{1}^{3} \log \left(\left|\frac{\tau_{2}}{2\left(\tau_{1}-\tau_{2}\right)}\right|\right)-\tau_{2}^{3} \log \left(\left|\frac{\tau_{1}}{2\left(\tau_{1}-\tau_{2}\right)}\right|\right)\right)}{\tau_{1}^{4} \tau_{2}^{4}} \\
& =\frac{1}{216} \tau_{\text {in }}^{6}\left[-18\left(\log \left(\frac{\tau}{4 \tau_{\text {in }}}\right)+1\right) \log \left(\frac{\tau}{\tau_{\text {in }}}\right)+6 \pi^{2}-29+30 \log (2)\right]+\mathcal{O}\left(\frac{\tau}{\tau_{\text {in }}}\right),
\end{aligned}
$$

$$
\begin{array}{r}
I_{10}=\int_{\tau_{\text {in }}}^{\tau} d \tau_{1} \int_{\tau_{\text {in }}}^{\tau_{1}} d \tau_{2} \frac{\left(\tau^{3}-\tau_{1}^{3}\right)^{3}\left(\log \left(-\frac{2 \eta \mu}{H \tau_{1}}\right)+\gamma\right)}{\tau_{1}^{4}} \delta\left(\tau_{1}-\tau_{2}\right)+\frac{\left(\tau^{3}-\tau_{1}^{3}\right)\left(\tau^{3}-\tau_{2}^{3}\right)^{2}}{\tau_{1}^{2} \tau_{2}^{2}\left(\tau_{1}-\tau_{2}\right)} \theta\left(\tau_{1}-\tau_{2}-\eta\right) \\
\left.=\frac{1}{6} \tau_{\text {in }}^{6}\left(\log \left(\frac{2 \mu}{H}\right)+\gamma-1\right)+\mathcal{O}\left(\frac{\tau}{\tau_{\text {in }}}\right) . \quad \text { (B. } 10\right)
\end{array}
$$


This appendix collects various minor results obtained during the first part of my work. They represents a reinforcement of the outcomes shown in this thesis.

In the first section the tadpole amplitude in Minkowski spacetime is fully Fourier transformed in momentum spacetime in order to obtain a quantity that can be compared to the analogous results in the literature. The next section compares the results of the thesis about the two-point function with those obtained using the dimensional regularization prescription both in Minkowski and de-Sitter spacetime. The comparison of the Minkowski tadpole with the analogous in-out results follows. Subsequently the de-Sitter propagators and the tadpole amplitude is compared to the Minkowski results in the limit of a flat spacetime. Then the fish diagram for a massive theory in Minkowski spacetime is discussed. The last two sections are devoted to the discussion of the finite time dependence that was found in the renormalization of composite operators and to our consideration about the issue of defining the tadpole diagram as a product of two distributions.

\section{Fourier Transform of the tadpole diagram in Minkowski spacetime}

In cosmology a special class of spacetimes of particular interest is given by the expanding Friedmann Lemaittre Robertson Walker universe. In this case the spatial part describes a maximally symmetric space and one can still have a proper definition of the Fourier transform as it was discussed in Chapter 4.

Here we consider the Fourier transform of the tadpole diagram (5.11) in Minkowski spacetime. The full amplitude is a function of momentum and time $A_{\text {amp }}(k, t)$ with the 
additional dependence on the initial time $t_{\text {in }}$. The momentum variable can be Fourier transformed back in order to obtain a quantity depending only on the four-vector $x^{\mu}=\left(t, x^{i}\right)$

$$
\begin{aligned}
A(x) & =\frac{-i A_{\mathrm{amp}}}{4} \frac{1}{(2 \pi)^{3}} \int_{0}^{\infty} \int_{-1}^{1} \int_{0}^{2 \pi} k^{2} d k d \cos \theta d \phi \frac{\sin \left(\sqrt{k^{2}+m^{2}}\left(t-t_{\text {in }}\right)\right)^{2}}{\left(k^{2}+m^{2}\right)^{3 / 2}} e^{-i k r \cos \theta} \\
& =\frac{-i \pi A_{\mathrm{amp}}}{(2 \pi)^{3}} \int_{0}^{\infty} k^{2} d k \frac{\sin \left(\sqrt{k^{2}+m^{2}}\left(t-t_{\mathrm{in}}\right)\right)^{2}}{\left(k^{2}+m^{2}\right)^{3 / 2}} \frac{\sin (k r)}{k r}
\end{aligned}
$$

This integral cannot be solved analytically and we could not go further. Alternatively we Fourier transformed the time coordinate in order to get an amplitude where all the coordinate are in Fourier space

$$
\begin{aligned}
A(k, w)= & \frac{-i A_{\mathrm{amp}}}{4} \int_{-\infty}^{\infty} d t \frac{\sin \left(\sqrt{k^{2}+m^{2}}\left(t-t_{\mathrm{in}}\right)\right)^{2}}{\left(k^{2}+m^{2}\right)^{3 / 2}} e^{i w t} \\
= & \frac{i A_{\mathrm{amp}}}{16} \int_{-\infty}^{\infty} d t \frac{1}{\left(k^{2}+m^{2}\right)^{3 / 2}}\left(e^{2 i \sqrt{k^{2}+m^{2}}\left(t-t_{\mathrm{in}}\right)}+e^{-2 i \sqrt{k^{2}+m^{2}}\left(t-t_{\mathrm{in}}\right)}-2\right) e^{i w t} \\
= & \frac{i A_{\mathrm{amp}} \pi}{8} \frac{1}{\left(k^{2}+m^{2}\right)^{3 / 2}}\left[\delta\left(w+2 \sqrt{k^{2}+m^{2}}\right) e^{-2 i \sqrt{k^{2}+m^{2}} t_{\mathrm{in}}}\right. \\
& \left.\quad+\delta\left(w-2 \sqrt{k^{2}+m^{2}}\right) e^{2 i \sqrt{k^{2}+m^{2}} t_{\mathrm{in}}}-2 \delta(w)\right] .
\end{aligned}
$$

The found amplitude in the full momentum space has to be compared to the analogous results in the literature [84]. It should be pointed-out that the Fourier transform does not cancel the dependence on the initial state which is an intrinsic contribution due to the fact that the vacuum state is not an eigenstate of the interacting theory. As discussed in Chapter 6 we expect that in the adiabatic limit and with a different interaction profile the time dependence is suppressed.

\section{Dimensional Regularization of the tadpole diagram}

Dimensional regularization is a method that was introduced in $[159,160]$ for regularizing divergent integrals in particle physics. The idea is to perform an analytic continuation of the number of spacetime dimensions $n$ where the integral can be performed. The result will be given as a Laurent expansion about the value $n-3^{1}$. Renormalization

\footnotetext{
${ }^{1}$ In the closed-time-path formalism the poles are powers of $(n-3)^{-1}$, because we are integrating over the 3-momentum $p$.
} 
consists in subtracting the poles from the regularized integral in order to obtain a finite quantity in the limit $n \rightarrow 3$.

In the following the one-loop amplitude is regularized with dimensional regularization in Minkowski and de-Sitter spacetime in order to test the renormalization procedure for a different prescription.

\section{Dimensional Regularization in Minkowski spacetime}

In dimensional regularization the loop integral in Minkowski spacetime is generalized to

$$
\left[\left(\frac{-i \lambda}{2}\right) \int \frac{d p^{3}}{(2 \pi)^{3}} F\left(w_{p}, t_{1}, t_{1}\right)\right] \rightarrow\left[\left(\frac{-i \lambda \mu^{3-n}}{2}\right) \int \frac{d p^{n}}{(2 \pi)^{n}} F\left(w_{p}, t_{1}, t_{1}\right)\right]
$$

where $w_{p}=\sqrt{p^{2}+m^{2}}, \mu$ is an arbitrary mass scale, and $n<3$ is chosen in order to have a finite integral. The found amplitude is consistent with analogous results in the literature [84].

$$
\begin{aligned}
A_{\mathrm{amp}}=\left(\frac{-i \lambda \mu^{3-n}}{2}\right) \int \frac{d p^{n}}{(2 \pi)^{n}} & \frac{1}{2 \sqrt{p^{2}+m^{2}}} \\
& =\frac{-i \lambda m^{2}}{32 \pi^{2}}\left(\frac{2}{n-3}-1+\gamma+\log \left(\frac{m^{2}}{4 \pi \mu^{2}}\right)\right) .
\end{aligned}
$$

\section{Dimensional Regularization in de-Sitter spacetime}

The same calculation is performed in de-Sitter spacetime. The ultraviolet contribution to the de-Sitter tadpole in eq. (5.16) is analytically continued to a spacetime of dimension $n$

$$
\frac{-i \lambda}{2 H^{4} \tau_{1}^{4}} \int_{M a\left(\tau_{1}\right)}^{\infty} d^{3} p\left(\frac{1}{p^{3}}+\frac{\tau_{1}^{2}}{p}\right) \rightarrow \frac{-i \lambda \mu^{3-n}}{4 H^{4} \tau_{1}^{4}} \int_{M a\left(\tau_{1}\right)}^{\infty} \frac{d^{n} p}{(2 \pi)^{n}}\left(\frac{1}{p^{3}}+\frac{\tau_{1}^{2}}{p}\right) .
$$

Using the spherical symmetry of the integral, the volume element can be simplified to

$$
\int d^{n} p=\int p^{n-1} \frac{2 \pi^{n / 2}}{\Gamma(n / 2)} d p
$$

Therefore the integral is regulated to a Laurent expansion about the pole in $n-3$

$$
\frac{i \lambda}{16 \pi^{2} H^{4} \tau_{1}^{4}}\left(\frac{2}{n-3}-2+\gamma+\log \left(\frac{M^{2} a\left(\tau_{1}\right)^{2}}{\pi \mu^{2}}\right)\right) .
$$


After having included the infrared contribution given in eq. (5.15), where the IR divergence was cured with the mass cutoff $\epsilon$, the full regulated integral reads

$$
\frac{-i \lambda}{16 \pi^{2} H^{4} \tau_{1}^{4}}\left(\frac{1}{\epsilon}-\frac{2}{n-3}+2-\gamma+\log \left(\pi \mu^{2} \tau_{1}^{2}\right)\right) .
$$

The simple pole in $n-3$ consistently represents the logarithmic divergence that we found with the cutoff prescription. The quadratic divergence is not intercepted by dimensional regularization. In the thesis divergent integrals were regulated using a cutoff in momentum space because it is a better prescription for analytic and numerical purposes and it physically represents our ignorance of physics of very high energy scales.

\section{Comparison with the in-out approach}

In this section the results obtained in the Schwinger and Keldysh formalism with the Hadamard propagators are compared with those given in the literature with the Feynman propagators. They are connected through the simple relation

$$
G^{F}=i\langle\mathrm{~T}\{\phi(x) \phi(y)\}\rangle=\frac{1}{2}\left(G^{R}+G^{A}\right)+i F .
$$

The sum of all contributions up to first order in $\lambda$ for a quartic interaction scalar field theory is

$$
\begin{aligned}
&\left\langle\mathrm{T}\left\{\phi\left(x, t_{a}\right) \phi\left(y, t_{b}\right)\right\}\right\rangle_{\mathrm{CTP}}=-i G_{(0)}^{F}+\left(\frac{-\lambda m^{2}}{128 \pi^{2} w_{k}^{3}}\right) C \times \\
& \times\left\{-\cos \left(w_{k}\left(t_{a}+t_{b}-2 t_{i n}\right)\right)+\theta\left(t_{b}-t_{a}\right) e^{i w_{k}\left(t_{a}-t_{b}\right)}\left[1-i w_{k}\left(t_{a}-t_{b}\right)\right]\right. \\
&\left.+\theta\left(t_{a}-t_{b}\right) e^{-i w_{k}\left(t_{a}-t_{b}\right)}\left[1-i w_{k}\left(t_{b}-t_{a}\right)\right]\right\}+\mathcal{O}\left(\lambda^{2}\right)
\end{aligned}
$$

where $G_{(0)}^{F}$ is the tree-level Feynman propagator and $C$ is a constant that represents the renormalization freedom since a different scheme is used in order to have a non-vanishing contribution.

It is interesting to compare this expression with the traditional tadpole in the in-out approach. In order to have similar quantities, we consider the expression in the 
basis of time and momentum. The result is

$$
\begin{gathered}
\left\langle\mathrm{T}\left\{\phi\left(x, t_{a}\right) \phi\left(y, t_{b}\right)\right\}\right\rangle_{k, t}^{(1)}=\left(\frac{\lambda m^{2}}{32 \pi^{2}}\right) C \int \frac{d w}{2 \pi}\left(\frac{1}{k^{2}+m^{2}+i \epsilon}\right)^{2} e^{-i w\left(t_{a}-t_{b}\right)} \\
=\left(\frac{\lambda m^{2}}{64 \pi^{3}}\right) C\left[-\theta\left(t_{a}-t_{b}\right) \operatorname{Res}_{w=w_{k}+i \epsilon}\left(\frac{1}{-w^{2}+w_{k}^{2}+i \epsilon}\right)^{2} e^{-i w\left(t_{a}-t_{b}\right)}\right. \\
\left.+\theta\left(t_{b}-t_{a}\right) \operatorname{Res}_{w=-w_{k}+i \epsilon}\left(\frac{1}{-w^{2}+w_{k}^{2}+i \epsilon}\right)^{2} e^{-i w\left(t_{a}-t_{b}\right)}\right] \\
=\left(\frac{\lambda m^{2}}{128 \pi^{2} w_{k}^{3}}\right) C\left[\theta\left(t_{a}-t_{b}\right) e^{-i w_{k}\left(t_{a}-t_{b}\right)}\left(1-i w_{k}\left(t_{b}-t_{a}\right)\right)\right. \\
\left.+\theta\left(t_{b}-t_{a}\right) e^{i w_{k}\left(t_{a}-t_{b}\right)}\left(1-i w_{k}\left(t_{a}-t_{b}\right)\right)\right] .
\end{gathered}
$$

It is interesting to note that eqs. (C.10) and (C.11) differ only by a periodic term that oscillates with a frequency of $w_{k}$.

$$
\left(\frac{\lambda m^{2}}{128 \pi^{2}}\right) C \frac{\cos \left(w_{k}\left(t_{a}+t_{b}-2 t_{\text {in }}\right)\right)}{w_{k}^{3}} .
$$

The origin of the time dependence was analyzed in Chapter 6, where it was shown that this contribution vanishes in the adiabatic limit, i.e. the analogous result in the in-out approach is consistently recovered.

\section{Comparison between the de-Sitter and the Minkowski propagators}

In the following section the zero-order expansion in the Hubble constant of the massless de-Sitter propagators (4.34) is given in order to show that the correct Minkowski propagators are recovered in the limit of flat FRW universe. Indeed, the Taylor expansions

$$
\begin{aligned}
& F\left(k, \tau_{1}, \tau_{2}\right)= \frac{H^{2}}{2 k^{3}}\left[\left(1+k^{2} \tau_{1} \tau_{2}\right) \cos \left(k\left(\tau_{1}-\tau_{2}\right)\right)+k\left(\tau_{1}-\tau_{2}\right) \sin \left(k\left(\tau_{1}-\tau_{2}\right)\right)\right] \\
&= \frac{H^{2}}{2 k^{3}}\left[\left(1+\frac{k^{2}}{H^{2}} e^{-H\left(t_{1}-t_{2}\right)}\right) \cos \left(\frac{k}{H}\left(e^{-H t_{2}}-e^{-H t_{1}}\right)\right)\right. \\
&\left.\quad+\frac{k}{H}\left(e^{-H t_{2}}-e^{-H t_{1}}\right) \sin \left(\frac{k}{H}\left(e^{-H t_{2}}-e^{-H t_{1}}\right)\right)\right] \\
&=\frac{H^{2}}{2 k^{3}} \frac{k^{2}}{H^{2}} \cos k\left(t_{1}-t_{2}\right)+\mathcal{O}(H)=\frac{1}{2 k} \cos k\left(t_{1}-t_{2}\right)+\mathcal{O}(H)
\end{aligned}
$$


and

$$
\begin{aligned}
& G_{R}\left(k, \tau_{1}, \tau_{2}\right)= \theta\left(\tau_{1}-\tau_{2}\right) \frac{H^{2}}{k^{3}}\left[\left(1+k^{2} \tau 1 \tau 2\right) \sin \left(k\left(\tau_{1}-\tau_{2}\right)\right)-k\left(\tau_{1}-\tau_{2}\right) \cos \left(k\left(\tau_{1}-\tau_{2}\right)\right)\right] \\
&=\theta\left(t_{1}-t_{2}\right) \frac{H^{2}}{k^{3}}\left[\left(1+\frac{k^{2}}{H^{2}} e^{-H\left(t_{1}-t_{2}\right)}\right) \sin \left(\frac{k}{H}\left(e^{-H t_{2}}-e^{-H t_{1}}\right)\right) \quad(\text { C. } 14)\right. \\
&\left.-\frac{k}{H}\left(e^{-H t_{2}}-e^{-H t_{1}}\right) \cos \left(\frac{k}{H}\left(e^{-H t_{2}}-e^{-H t_{1}}\right)\right)\right] \quad(\mathrm{C} .16) \\
&= \theta\left(t_{1}-t_{2}\right) \frac{H^{2}}{k^{3}} \frac{k^{2}}{H^{2}} \sin k\left(t_{1}-t_{2}\right)+\mathcal{O}(H)=\theta\left(t_{1}-t_{2}\right) \frac{1}{k} \sin k\left(t_{1}-t_{2}\right)+\mathcal{O}(H) .
\end{aligned}
$$

consistently give the Minkowski limit at zeroth-order in the Hubble constant. Moreover it was checked that the de-Sitter tadpole given in eq. (5.22) gives the correct limit (5.5) for a vanishing Hubble constant $H$, i.e.

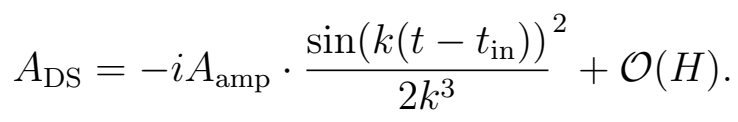

\section{The massive fish diagram in Minkowski spacetime}

In the following the divergences that arise in the one-loop calculations for the fourpoint functions are calculated for a massive theory with a quartic self-interaction in Minkowski spacetime. As for the massless scalar field theory, only the divergences of the singular contributions in Figure 5.8 are studied.

For the two diagrams, only the following momentum integral has to be renormalized

$$
\int \frac{d^{3} p_{1}}{(2 \pi)^{3}} \int \frac{d^{3} p_{2}}{(2 \pi)^{3}} F\left(w_{p_{1}}, t_{1}, t_{2}\right) G^{R}\left(w_{p_{2}}, t_{1}, t_{2}\right)(2 \pi)^{3} \delta\left(k_{1}+k_{2}-p_{1}-p_{2}\right),
$$

where $w_{p_{i}}=\sqrt{p_{i}^{2}+m^{2}}$ and $F$ and $G^{R}$ are the massive propagators in the Schwinger basis. In order to extrapolate an analytic expression from the integral, the external momenta are supposed to be small and the approximation $w_{p_{1}-k}=w_{p_{1}}$ was used, where $k=k_{1}+k_{2}$. The integral is simplified and reads

$$
\int \frac{d^{3} p}{(2 \pi)^{3}} F\left(w_{p}, t_{1}, t_{2}\right) G^{R}\left(w_{p}, t_{1}, t_{2}\right)
$$


or more explicitly

$$
\int d p\left(\frac{p^{2}}{8 \pi^{2}\left(m^{2}+p^{2}\right)}\right) \theta\left(t_{1}-t_{2}\right) \sin \left(2 \sqrt{m^{2}+p^{2}}\left(t_{1}-t_{2}\right)\right) .
$$

The integral is divergent and cannot be integrated analytically. Nevertheless, it can be shown that the integral has the same singular behavior of the following integral, i.e. they differ only by finite terms

$$
\begin{aligned}
\int d p & \left(\frac{p}{8 \pi^{2} \sqrt{\left(m^{2}+p^{2}\right)}}\right) \theta\left(t_{1}-t_{2}\right) \sin \left(2 \sqrt{m^{2}+p^{2}}\left(t_{1}-t_{2}\right)\right) \\
& =\left(\frac{1}{16 \pi^{2}}\right) \theta\left(t_{1}-t_{2}\right)\left[\frac{\cos \left(2 m\left(t_{1}-t_{2}\right)\right)-\cos \left(2 \sqrt{m^{2}+\Lambda^{2}}\left(t_{1}-t_{2}\right)\right)}{t_{1}-t_{2}}\right] .
\end{aligned}
$$

The last expression can be integrated analytically. Inspired by the massless case in eq. (5.35) one finds the explicit form

$$
\begin{array}{r}
\theta\left(t_{1}-t_{2}\right)\left[\frac{\cos \left(2 m\left(t_{1}-t_{2}\right)\right)-\cos \left(2 \sqrt{m^{2}+\Lambda^{2}}\left(t_{1}-t_{2}\right)\right)}{t_{1}-t_{2}}\right] \\
=\theta(-\eta+\Delta t) \frac{\cos (2 m \Delta t)}{\Delta t}+\frac{1}{2} \delta(\Delta t) \log \left(1+\left(\frac{\Lambda}{m}\right)^{2}\right),
\end{array}
$$

where $\eta$ is a time regulator and is sent to zero at the end. The equality holds in the distributional sense. The final regulated expression for the amputated diagram is finally given by

$$
\left(\frac{1}{16 \pi^{2}}\right)\left[\theta(\Delta t) \frac{\cos (2 m \Delta t)}{\Delta t}+\delta(\Delta t) \log \left(\frac{\Lambda}{m}\right)\right] .
$$

It should be noted that the the massive fish diagram in Minkowski spacetime is also regulated in the minimal subtraction scheme by the coupling constant counter-term $\delta \lambda$ defined for the massless theory in eq. (5.36). This term is universal and could be used in order to regulate the four-point function in de-Sitter space also for the massive theory.

\section{Comment on the finite dependence of renormalized composite operators}

In quantum field theory the product of operators at coinciding points are called composite operators and are singular. These operators appear often in physics, as in 
the stress-energy tensor or in the number operator. They are of particular interest because these quantities can be measured. In [36] the non-local effects depending on the initial state were computed in the case of a two scalar field theory in de-Sitter spacetime similar to the hybrid model (7.17) that we described in this work.

They found that the renormalized composite operators had a local logarithmic dependence on the scale factor, similar to the one we found in the amputated two-point function in eq. (5.18) plus a non-local oscillatory contribution. In the limit of a flat spacetime they found that the oscillation arising from the external propagators are proportional to the kernel function $K\left(t-t_{\text {in }}\right)$, where

$$
K(t)=\int \frac{d^{3} k}{(2 \pi)^{3}} \frac{\cos \left(2 w_{k} t\right)}{\left(w_{k}\right)^{3}}
$$

$w_{k}=\sqrt{k^{2}+m^{2}}$, and $m$ is the field's mass. This extra term has an amplitude which decreases faster than $\left[m\left(t-t_{\text {in }}\right)\right]^{-3 / 2}$ and consistently disappears for $t-t_{\text {in }} \rightarrow \infty$ where the Poincaré symmetry is restored.

This result is fully consistent with our discussion about the initial time dependence of the non-local contribution and the profile dependence in Chapter 6 .

\section{Comment on the tadpole seen as an extension of distributions}

From the point of view of functions the tadpole is an ill-defined quantity, i.e. $\Delta_{F}(x-x)=$ $\Delta_{F}(0)$ is divergent. In momentum space it is given by $\int d^{4} p \frac{1}{p^{2}}$ and has a quadratic divergence that can be renormalized to 0 after a proper choice of the counter-term. This renormalization, which in momentum space looks similar to the renormalization techniques used for other diagrams, is in fact different when we try an extension in the language of distributions. The reason is that $\Delta_{F}(0)$ is ill defined everywhere. To try to avoid this problem, one can see $\Delta_{F}(0)$ as an extension to the diagonal of the product of two distributions, like for example

$$
\Delta_{F}(0)=\int d^{4} x \delta^{(4)}(x) \Delta_{F}(x)
$$

In this case, $\delta^{4}(x) \Delta_{F}(x)$ is identically zero for test functions whose support does not include the origin. This means that the singular order of eq. (C.26) is $-\infty$ and the unique extension is the null function. In other words, this would suggest that $\left[\Delta_{F}(0)\right]_{\mathrm{ren}}=0$. 
We point out that in the algebraic approach, tadpoles are not considered since the interaction is always given as the Wick ordering of polynomial functions of fields. This means that after a proper choice of normal ordering, : $\phi^{2}(x)$ : becomes regular. In fact in the Dyson representation of the S-matrix, $\Delta_{F}(0)$ never appears. 
[1] Alan H. Guth. "The Inflationary Universe: A Possible Solution to the Horizon and Flatness Problems". In: Phys. Rev. D23 (1981), pp. 347-356.

[2] Andreas Albrecht and Paul J. Steinhardt. "Cosmology for Grand Unified Theories with Radiatively Induced Symmetry Breaking". In: Phys. Rev. Lett. 48 (1982), pp. 1220-1223.

[3] Andrei D. Linde. "A New Inflationary Universe Scenario: A Possible Solution of the Horizon, Flatness, Homogeneity, Isotropy and Primordial Monopole Problems". In: Phys. Lett. 108B (1982), pp. 389-393.

[4] Viatcheslav F. Mukhanov and G. V. Chibisov. "Quantum Fluctuations and a Nonsingular Universe". In: JETP Lett. 33 (1981). [Pisma Zh. Eksp. Teor. Fiz.33,549(1981)], pp. 532-535.

[5] Viatcheslav F. Mukhanov, H. A. Feldman, and Robert H. Brandenberger. "Theory of cosmological perturbations. Part 1. Classical perturbations. Part 2. Quantum theory of perturbations. Part 3. Extensions". In: Phys. Rept. 215 (1992), pp. 203-333.

[6] P. A. R. Ade et al. "Planck 2015 results. XX. Constraints on inflation". In: Astron. Astrophys. 594 (2016), A20. arXiv: 1502.02114 [astro-ph.C0].

[7] David H. Lyth and Antonio Riotto. "Particle physics models of inflation and the cosmological density perturbation". In: Phys. Rept. 314 (1999), pp. 1-146. arXiv: hep-ph/9807278 [hep-ph].

[8] Jerome Martin, Christophe Ringeval, and Vincent Vennin. "Encyclopædia Inflationaris". In: Phys. Dark Univ. 5-6 (2014), pp. 75-235. arXiv: 1303.3787 [astro-ph. CO]. 
[9] Alexei A. Starobinsky. "Spectrum of adiabatic perturbations in the universe when there are singularities in the inflation potential". In: JETP Lett. 55 (1992). [Pisma Zh. Eksp. Teor. Fiz.55,477(1992)], pp. 489-494.

[10] Jerome Martin and Christophe Ringeval. "Exploring the superimposed oscillations parameter space". In: JCAP 0501 (2005), p. 007. arXiv: hep-ph/0405249 [hep-ph].

[11] Paul Hunt and Subir Sarkar. "Multiple inflation and the WMAP 'glitches"'. In: Phys. Rev. D70 (2004), p. 103518. arXiv: astro-ph/0408138 [astro-ph].

[12] Masahiro Kawasaki, Fuminobu Takahashi, and Tomo Takahashi. "Making waves on CMB power spectrum and inflaton dynamics". In: Phys. Lett. B605 (2005), pp. 223-227. arXiv: astro-ph/0407631 [astro-ph].

[13] Laila Alabidi. "Non-gaussianity for a Two Component Hybrid Model of Inflation". In: JCAP 0610 (2006), p. 015. arXiv: astro-ph/0604611 [astro-ph].

[14] Laura Covi et al. "Inflation and WMAP three year data: Features have a Future!" In: Phys. Rev. D74 (2006), p. 083509. arXiv: astro-ph/0606452 [astro-ph].

[15] Jan Hamann et al. "New Constraints on Oscillations in the Primordial Spectrum of Inflationary Perturbations". In: Phys. Rev. D76 (2007), p. 023503. arXiv: astro-ph/0701380 [astro-ph].

[16] Xingang Chen. "Primordial Features as Evidence for Inflation". In: JCAP 1201 (2012), p. 038. arXiv: 1104.1323 [hep-th].

[17] Juan Martin Maldacena. "Non-Gaussian features of primordial fluctuations in single field inflationary models". In: JHEP 05 (2003), p. 013. arXiv: astro-ph/ 0210603 [astro-ph].

[18] N. Bartolo et al. "Non-Gaussianity from inflation: Theory and observations". In: Phys. Rept. 402 (2004), pp. 103-266. arXiv: astro-ph/0406398 [astro-ph].

[19] Jens Chluba, Jan Hamann, and Subodh P. Patil. "Features and New Physical Scales in Primordial Observables: Theory and Observation". In: Int. J. Mod. Phys. D24.10 (2015), p. 1530023. arXiv: 1505.01834 [astro-ph.C0].

[20] T. S. Bunch and P. C. W. Davies. "Quantum Field Theory in de Sitter Space: Renormalization by Point Splitting". In: Proc. Roy. Soc. Lond. A360 (1978), pp. $117-134$.

[21] Andreas Ringwald. "Dynamical Evolution Equation of the Scalar Field During Reheating in the New Inflationary Universe". In: Z. Phys. C34 (1987), p. 481. 
[22] D. Boyanovsky, H. J. de Vega, and R. Holman. "Nonequilibrium evolution of scalar fields in FRW cosmologies I". In: Phys. Rev. D49 (1994), pp. 2769-2785. arXiv: hep-ph/9310319 [hep-ph].

[23] D. Boyanovsky et al. "Scalar field dynamics in Friedman-Robertson-Walker space-times". In: Phys. Rev. D56 (1997), pp. 1939-1957. arXiv: hep-ph/9703327 [hep-ph].

[24] D. Boyanovsky et al. "Out-of-equilibrium dynamics of an inflationary phase transition". In: Phys. Rev. D55 (1997), pp. 3373-3388. arXiv: hep-ph/9610396 [hep-ph].

[25] G. Pinter. "Finite renormalizations in the Epstein Glaser framework and renormalization of the S matrix of Phi**4-theory". In: Annalen Phys. 10 (2001), pp. 333-363. arXiv: hep-th/9911063 [hep-th].

[26] Dirk Prange. "Epstein-Glaser renormalization and differential renormalization". In: J.Phys. A32 (1999), pp. 2225-2238. arXiv: hep-th/9710225 [hep-th].

[27] Jurgen Baacke and Carsten Patzold. "Renormalization of the nonequilibrium dynamics of fermions in a flat FRW universe". In: Phys. Rev. D62 (2000), p. 084008. arXiv: hep-ph/9912505 [hep-ph].

[28] Steven Weinberg. "Quantum contributions to cosmological correlations". In: Phys. Rev. D72 (2005), p. 043514. arXiv: hep-th/0506236 [hep-th].

[29] Steven Weinberg. "Quantum contributions to cosmological correlations. II. Can these corrections become large?" In: Phys. Rev. D74 (2006), p. 023508. arXiv: hep-th/0605244 [hep-th].

[30] Ante Bilandzic and Tomislav Prokopec. "Quantum radiative corrections to slow-roll inflation". In: Phys. Rev. D76 (2007), p. 103507. arXiv: 0704.1905 [astro-ph].

[31] Szabolcs Borsanyi and Urko Reinosa. "Renormalised nonequilibrium quantum field theory: Scalar fields". In: Phys. Rev. D80 (2009), p. 125029. arXiv: 0809. 0496 [hep-th].

[32] Leonardo Senatore and Matias Zaldarriaga. "On Loops in Inflation". In: JHEP 12 (2010), p. 008. arXiv: 0912.2734 [hep-th].

[33] Jurgen Baacke, Laura Covi, and Nina Kevlishvili. "Coupled scalar fields in a flat FRW universe: Renormalisation". In: JCAP 1008 (2010), p. 026. arXiv: 1006. 2376 [hep-ph]. 
[34] Tommi Markkanen and Anders Tranberg. "A Simple Method for One-Loop Renormalization in Curved Space-Time". In: JCAP 1308 (2013), p. 045. arXiv: 1303.0180 [hep-th].

[35] Matti Herranen, Tommi Markkanen, and Anders Tranberg. "Quantum corrections to scalar field dynamics in a slow-roll space-time". In: (2013). arXiv: 1311.5532 [hep-ph].

[36] Simone Dresti and Antonio Riotto. "Renormalization of Composite Operators in time-dependent Backgrounds". In: Nucl.Phys. B874 (2013), pp. 792-807. arXiv: 1305.6401 [hep-ph] .

[37] Thomas-Paul Hack. "Cosmological Applications of Algebraic Quantum Field Theory in Curved Spacetimes". In: (2015). arXiv: 1506.01869 [gr-qc].

[38] Antoine Géré, Thomas-Paul Hack, and Nicola Pinamonti. "An analytic regularisation scheme on curved spacetimes with applications to cosmological spacetimes". In: (2015). arXiv: 1505.00286 [math-ph].

[39] David Seery. "Infrared effects in inflationary correlation functions". In: Class. Quant. Grav. 27 (2010), p. 124005. arXiv: 1005.1649 [astro-ph.c0].

[40] Andrei D. Linde. "Chaotic Inflation". In: Phys. Lett. 129B (1983), pp. 177-181.

[41] Andrei D. Linde. "Hybrid inflation". In: Phys. Rev. D49 (1994), pp. 748-754. arXiv: astro-ph/9307002 [astro-ph].

[42] David Seery, James E. Lidsey, and Martin S. Sloth. "The inflationary trispectrum". In: JCAP 0701 (2007), p. 027. arXiv: astro-ph/0610210 [astro-ph].

[43] Christian T. Byrnes, Misao Sasaki, and David Wands. "The primordial trispectrum from inflation". In: Phys. Rev. D74 (2006), p. 123519. arXiv: astro-ph/ 0611075 [astro-ph].

[44] J. A. Peacock. Cosmological Physics. Jan. 1999.

[45] E. W. Kolb and M. S. Turner. The early universe. 1990.

[46] S. Dodelson. Modern cosmology. 2003.

[47] Julien Lesgourgues. "An Overview of cosmology". In: (2004). arXiv: astro-ph/ 0409426 [astro-ph].

[48] Alexei A. Starobinsky. "A New Type of Isotropic Cosmological Models Without Singularity". In: Phys. Lett. B91 (1980). [,771(1980)], pp. 99-102.

[49] A. Friedmann. "On the Possibility of a world with constant negative curvature of space". In: Z. Phys. 21 (1924). [Gen. Rel. Grav.31,2001(1999)], pp. 326-332. 
[50] Georges Lemaitre. "The Expanding Universe". In: Mon. Not. Roy. Astron. Soc. 91 (1931), pp. 490-501.

[51] Georges Lemaitre. "A homogeneous universe of constant mass and increasing radius accounting for the radial velocity of extra-galactic nebulae". In: Mon. Not. Roy. Astron. Soc. 91 (1931), pp. 483-490.

[52] G. Lemaitre. "The expanding universe". In: Gen. Rel. Grav. 29 (1997). [Annales Soc. Sci. Bruxelles A53,51(1933)], pp. 641-680.

[53] H. P. Robertson. "Kinematics and World-Structure". In: Astrophys. J. 82 (1935), pp. 284-301.

[54] H. P. Robertson. "Kinematics and World-Structure. 2". In: Astrophys. J. 83 (1935), pp. 187-201.

[55] H. P. Robertson. "Kinematics and World-Structure. 3". In: Astrophys. J. 83 (1936), pp. 257-271.

[56] Arthur Geoffrey Walker. "On Milne's Theory of World-Structure". In: Proceedings of the London Mathematical Society 2.1 (1937), pp. 90-127.

[57] Isaac Newton. "1687". In: Philosophiae naturalis principia mathematica 3 (1995).

[58] Albert Einstein. "The Field Equations of Gravitation". In: Sitzungsber. Preuss. Akad. Wiss. Berlin (Math. Phys.) 1915 (1915), pp. 844-847.

[59] Albert Einstein. "On the General Theory of Relativity". In: Sitzungsber. Preuss. Akad. Wiss. Berlin (Math. Phys.) 1915 (1915). [Addendum: Sitzungsber. Preuss. Akad. Wiss. Berlin (Math. Phys.)1915,799(1915)], pp. 778-786.

[60] Albert Einstein. "The Foundation of the General Theory of Relativity". In: Annalen Phys. 49.7 (1916). [,65(1916)], pp. 769-822.

[61] Albert Einstein. "Approximative Integration of the Field Equations of Gravitation". In: Sitzungsber. Preuss. Akad. Wiss. Berlin (Math. Phys.) 1916 (1916), pp. 688-696.

[62] Albert Einstein. "Hamilton's Principle and the General Theory of Relativity". In: Sitzungsber. Preuss. Akad. Wiss. Berlin (Math. Phys.) 1916 (1916), pp. 11111116.

[63] Edwin Hubble. "A relation between distance and radial velocity among extragalactic nebulae". In: Proc. Nat. Acad. Sci. 15 (1929), pp. 168-173.

[64] Edwin Powell Hubble. The observational approach to cosmology. Clarendon Press Oxford, 1937. 
[65] W. de Sitter. "Einstein's theory of gravitation and its astronomical consequences, First Paper". In: Mon. Not. Roy. Astron. Soc. 76 (1916), pp. 699-728.

[66] W. de Sitter. "Einstein's theory of gravitation and its astronomical consequences, Second Paper". In: Mon. Not. Roy. Astron. Soc. 77 (1916), pp. 155-184.

[67] W. de Sitter. "Einstein's theory of gravitation and its astronomical consequences, Third Paper". In: Mon. Not. Roy. Astron. Soc. 78 (1917), pp. 3-28.

[68] A. Einstein and W. de Sitter. "On the Relation between the Expansion and the Mean Density of the Universe". In: Proc. Nat. Acad. Sci. 18 (1932), pp. 213-214.

[69] P. A. R. Ade et al. "Planck 2015 results. XIII. Cosmological parameters". In: Astron. Astrophys. 594 (2016), A13. arXiv: 1502.01589 [astro-ph.CO].

[70] V. Mukhanov. Physical Foundations of Cosmology. Oxford: Cambridge University Press, 2005. ISBN: 0521563984, 9780521563987.

[71] Scott Dodelson. Modern Cosmology. Amsterdam: Academic Press, 2003. ISBN: 9780122191411.

[72] David H. Lyth and Andrew R. Liddle. The primordial density perturbation: Cosmology, inflation and the origin of structure. 2009.

[73] A. Riotto. "Particle cosmology". In: High-energy physics. Proceedings, 5th CERN-Latin-American School, Recinto Quirama, Colombia, March 15-28, 2009. 2010. arXiv: 1010.2642 [hep-ph].

[74] G. V. Chibisov and Viatcheslav F. Mukhanov. "Quantum fluctuations in cosmology and galaxy formation". In: Second Seminar on Quantum Gravity Moscow, USSR, October 13-15, 1981. 1981, pp. 161-171.

[75] Alan H. Guth and S. Y. Pi. "Fluctuations in the New Inflationary Universe". In: Phys. Rev. Lett. 49 (1982), pp. 1110-1113.

[76] Alexei A. Starobinsky. "Dynamics of Phase Transition in the New Inflationary Universe Scenario and Generation of Perturbations". In: Phys. Lett. 117B (1982), pp. $175-178$.

[77] Milton Abramowitz and Irene A Stegun. Handbook of mathematical functions: with formulas, graphs, and mathematical tables. Vol. 55. Courier Corporation, 1964.

[78] James M. Bardeen. "Gauge Invariant Cosmological Perturbations". In: Phys. Rev. D22 (1980), pp. 1882-1905. 
[79] James M. Bardeen, Paul J. Steinhardt, and Michael S. Turner. "Spontaneous Creation of Almost Scale - Free Density Perturbations in an Inflationary Universe". In: Phys. Rev. D28 (1983), p. 679.

[80] Daniel Baumann. "Inflation". In: Physics of the large and the small, TASI 09, proceedings of the Theoretical Advanced Study Institute in Elementary Particle Physics, Boulder, Colorado, USA, 1-26 June 2009. 2011, pp. 523-686. arXiv: 0907.5424 [hep-th].

[81] Andrei D. Linde. "Inflationary Cosmology". In: Lect. Notes Phys. 738 (2008), pp. 1-54. arXiv: 0705.0164 [hep-th].

[82] Katherine Freese, Joshua A. Frieman, and Angela V. Olinto. "Natural inflation with pseudo - Nambu-Goldstone bosons". In: Phys. Rev. Lett. 65 (1990), pp. 3233-3236.

[83] David Wands. "Multiple field inflation". In: Lect. Notes Phys. 738 (2008), pp. 275-304. arXiv: astro-ph/0702187 [ASTRO-PH].

[84] Pierre Ramond. "Field theory, a modern primer". In: Front. Phys. 51 (1981). [Front. Phys.74,1(1989)], pp. 1-397.

[85] Michael E. Peskin and Daniel V. Schroeder. An Introduction to quantum field theory. Reading, USA: Addison-Wesley, 1995. ISBN: $9780201503975,0201503972$.

[86] Michele Maggiore. A modern introduction to quantum field theory. Vol. 12. Oxford University Press, 2005.

[87] L. H. Ford. "Quantum field theory in curved space-time". In: Particles and fields. Proceedings, 9th Jorge Andre Swieca Summer School, Campos do Jordao, Brazil, February 16-28, 199\%. 1997, pp. 345-388. arXiv: gr-qc/9707062 [gr-qc].

[88] Sergei Winitzki. "Elementary Introduction to Quantum Fields in Curved Spacetime". In: 2006.

[89] Stefan Hollands. "Trento lectures on quantum field theory on curved spacetimes". In: 2008.

[90] Klaus Fredenhagen and Katarzyna Rejzner. "Quantum field theory on curved spacetimes: Axiomatic framework and examples". In: J. Math. Phys. 57.3 (2016), p. 031101. arXiv: 1412.5125 [math-ph].

[91] N. D. Birrell and P. C. W. Davies. Quantum Fields in Curved Space. Cambridge Monographs on Mathematical Physics. Cambridge, UK: Cambridge Univ. Press, 1984. ISBN: 0521278589, 9780521278584, 9780521278584. 
[92] Leonard E. Parker and D. Toms. Quantum Field Theory in Curved Spacetime. Cambridge Monographs on Mathematical Physics. Cambridge University Press, 2009. ISBN: $9780521877879,9780521877879,9780511601552$.

[93] Bernard J. Carr and S. W. Hawking. "Black holes in the early Universe". In: Mon. Not. Roy. Astron. Soc. 168 (1974), pp. 399-415.

[94] S. W. Hawking. "Black hole explosions". In: Nature 248 (1974), pp. 30-31.

[95] S. W. Hawking. "Particle Creation by Black Holes". In: Commun. Math. Phys. 43 (1975). [,167(1975)], pp. 199-220.

[96] W. G. Unruh. "Notes on black hole evaporation". In: Phys. Rev. D14 (1976), p. 870 .

[97] Steven Weinberg. "New approach to the renormalization group". In: Phys. Rev. D8 (1973), pp. 3497-3509.

[98] Gerard 't Hooft. "Dimensional regularization and the renormalization group". In: Nucl. Phys. B61 (1973), pp. 455-468.

[99] Leonard Parker. "Particle creation and particle number in an expanding universe". In: J. Phys. A45 (2012), p. 374023. arXiv: 1205.5616 [astro-ph.CO].

[100] Leonard Parker and S. A. Fulling. "Adiabatic regularization of the energy momentum tensor of a quantized field in homogeneous spaces". In: Phys. Rev. D9 (1974), pp. 341-354.

[101] Tommi Markkanen. "Applications of Curved Space Field Theory to Simple Scalar Field Models of Inflation". PhD thesis. Helsinki U., 2014.

[102] S. A. Fulling and L. Parker. "Renormalization in the theory of a quantized scalar field interacting with a robertson-walker spacetime". In: Annals Phys. 87 (1974), pp. 176-204.

[103] S. A. Fulling, Leonard Parker, and B. L. Hu. "Conformal energy-momentum tensor in curved spacetime: Adiabatic regularization and renormalization". In: Phys. Rev. D10 (1974), pp. 3905-3924.

[104] T. S. Bunch. "Adiabatic regularization for scalar fields with arbitrary coupling to the scalar curvature". In: J. Phys. A13 (1980), pp. 1297-1310.

[105] Leonard Parker and David Toms. Quantum field theory in curved spacetime: quantized fields and gravity. Cambridge University Press, 2009.

[106] Tommi Markkanen and Anders Tranberg. "Quantum Corrections to Inflaton and Curvaton Dynamics". In: JCAP 1211 (2012), p. 027. arXiv: 1207.2179 [gr-qc]. 
[107] H. Epstein and V. Glaser. "The Role of locality in perturbation theory". In: Ann. Inst. H. Poincare Phys. Theor. A19 (1973), pp. 211-295.

[108] C. Baer and K. Fredenhagen. "Quantum field theory on curved spacetimes". In: Lect. Notes Phys. 786 (2009), pp. 1-155.

[109] Walter Rudin. "Functional analysis 2nd ed". In: International Series in Pure and Applied Mathematics. McGraw-Hill, Inc., New York (1991).

[110] R Stora. "Differential algebras in Lagrangean field theory. ETH-Zürich Lectures, January-February 1993; Popineau, G. and Stora, R.: A pedagogical remark on the main theorem of perturbative renormalization theory". In: Unpublished preprint (1982).

[111] R. Brunetti and K. Fredenhagen. "Interacting quantum fields in curved space: Renormalizability of phi**4". In: (1997). arXiv: gr-qc/9701048 [gr-qc].

[112] Romeo Brunetti and Klaus Fredenhagen. "Microlocal analysis and interacting quantum field theories: Renormalization on physical backgrounds". In: Commun. Math. Phys. 208 (2000), pp. 623-661. arXiv: math-ph/9903028 [math-ph].

[113] O. Steinmann. "Perturbation theory in the axiomatic approach". In: Proceedings, Congress on Renormalization Theory: Marseille, France, Jun 14-18, 1971. 1971, pp. 22-31.

[114] Dirk Prange. "Lorentz covariance in Epstein-Glaser renormalization". In: (1999). arXiv: hep-th/9904136 [hep-th].

[115] Romeo Brunetti and Klaus Fredenhagen. "Interacting quantum fields on a curved background". In: Mathematical physics. Proceedings, 12th International Congress, ICMP'97, Brisbane, Australia, July 13-19, 1997. 1997, pp. 186-190. arXiv: hep-th/9709011 [hep-th] .

[116] Romeo Brunetti and Klaus Fredenhagen. "Quantum Field Theory on Curved Backgrounds". In: (2009). arXiv: 0901.2063 [gr-qc].

[117] R. Brunetti, K. Fredenhagen, and M. Kohler. "The Microlocal spectrum condition and Wick polynomials of free fields on curved space-times". In: Commun. Math. Phys. 180 (1996), pp. 633-652. arXiv: gr-qc/9510056 [gr-qc].

[118] M. J. Radzikowski. "Micro-local approach to the Hadamard condition in quantum field theory on curved space-time". In: Commun. Math. Phys. 179 (1996), pp. 529-553.

[119] Lars Hörmander. "Fourier integral operators. I". In: Acta mathematica 127.1 (1971), p. 79. 
[120] Johannes J Duistermaat and Lars Hörmander. "Fourier integral operators. II". In: Acta mathematica 128.1 (1972), pp. 183-269.

[121] M. Maggiore. A modern introduction to quantum field theory. Oxford, 2006.

[122] P. Ramond and H. M. Fried. "Field Theory: A Modern Primer". In: Physics Today 35 (1982), p. 57.

[123] L. S. Brown. Quantum Field Theory. Aug. 1994.

[124] M. E. Peskin and D. V. Schroeder. An Introduction to Quantum Field Theory. Westview Press, 1995.

[125] Edward Witten. "Quantum gravity in de Sitter space". In: Strings 2001: International Conference Mumbai, India, January 5-10, 2001. 2001. arXiv: hep-th/0106109 [hep-th].

[126] Julian S. Schwinger. "Brownian motion of a quantum oscillator". In: J.Math.Phys. 2 (1961), pp. 407-432.

[127] L. V. Keldysh. "Diagram technique for nonequilibrium processes". In: Zh. Eksp. Teor. Fiz. 47 (1964). [Sov. Phys. JETP20,1018(1965)], pp. 1515-1527.

[128] Pradip M. Bakshi and Kalyana T. Mahanthappa. "Expectation value formalism in quantum field theory. 1." In: J. Math. Phys. 4 (1963), pp. 1-11.

[129] Pradip M. Bakshi and Kalyana T. Mahanthappa. "Expectation value formalism in quantum field theory. 2." In: J. Math. Phys. 4 (1963), pp. 12-16.

[130] E. Calzetta and B. L. Hu. "Nonequilibrium Quantum Fields: Closed Time Path Effective Action, Wigner Function and Boltzmann Equation". In: Phys. Rev. D37 (1988), p. 2878.

[131] Hael Collins, R. Holman, and Andreas Ross. "Effective field theory in timedependent settings". In: JHEP 02 (2013), p. 108. arXiv: 1208.3255 [hep-th].

[132] Alex Kamenev. Field theory of non-equilibrium systems. Cambridge University Press, 2011.

[133] H. Collins, R. Holman, and A. Ross. "Effective field theory in time-dependent settings". In: ArXiv e-prints (Aug. 2012). arXiv: 1208.3255 [hep-th].

[134] S. A. Fulling. "Aspects of Quantum Field Theory in Curved Space-time". In: London Math. Soc. Student Texts 17 (1989), pp. 1-315.

[135] Bruce Allen. "Vacuum States in de Sitter Space". In: Phys. Rev. D32 (1985), p. 3136 . 
[136] D. Boyanovsky, R. Holman, and S. Prem Kumar. "Inflaton decay in De Sitter space-time". In: Phys.Rev. D56 (1997), pp. 1958-1972. arXiv: hep-ph/9606208 [hep-ph] .

[137] D. Boyanovsky, Hector J. de Vega, and Norma G. Sanchez. "Quantum corrections to slow roll inflation and new scaling of superhorizon fluctuations". In: Nucl.Phys. B747 (2006), pp. 25-54. arXiv: astro-ph/0503669 [astro-ph].

[138] D. Boyanovsky, Hector J. de Vega, and Norma G. Sanchez. "Particle decay during inflation: Self-decay of inflaton quantum fluctuations during slow roll". In: Phys.Rev. D71 (2005), p. 023509. arXiv: astro-ph/0409406 [astro-ph].

[139] Mathematica Version 11.2 Champaign Wolfram Research Inc. In: (IL (2017)).

[140] Mark G. Jackson and Koenraad Schalm. "Model Independent Signatures of New Physics in the Inflationary Power Spectrum". In: Phys. Rev. Lett. 108 (2012), p. 111301. arXiv: 1007.0185 [hep-th].

[141] Mark G. Jackson and Koenraad Schalm. "Model-Independent Signatures of New Physics in Slow-Roll Inflation". In: (2011). arXiv: 1104.0887 [hep-th].

[142] Mark G. Jackson and Koenraad Schalm. "Model-Independent Signatures of New Physics in non-Gaussianity". In: (2012). arXiv: 1202.0604 [hep-th] .

[143] Meindert van der Meulen and Jan Smit. "Classical approximation to quantum cosmological correlations". In: JCAP 0711 (2007), p. 023. arXiv: 0707.0842 [hep-th].

[144] L. S. Brown. Quantum field theory. Cambridge University Press, 1994. ISBN: 9780521469463.

[145] Stefan Hollands and Robert M. Wald. "Local Wick polynomials and time ordered products of quantum fields in curved space-time". In: Commun. Math. Phys. 223 (2001), pp. 289-326. arXiv: gr-qc/0103074 [gr-qc] .

[146] Fedor L. Bezrukov and Mikhail Shaposhnikov. "The Standard Model Higgs boson as the inflaton". In: Phys. Lett. B659 (2008), pp. 703-706. arXiv: 0710. 3755 [hep-th].

[147] P. A. R. Ade et al. "Planck 2013 results. XXII. Constraints on inflation". In: Astron. Astrophys. 571 (2014), A22. arXiv: 1303.5082 [astro-ph.Co].

[148] Sidney R. Coleman and Erick J. Weinberg. "Radiative Corrections as the Origin of Spontaneous Symmetry Breaking". In: Phys. Rev. D7 (1973), pp. 1888-1910.

[149] G. R. Dvali, Q. Shafi, and Robert K. Schaefer. "Large scale structure and supersymmetric inflation without fine tuning". In: Phys. Rev. Lett. 73 (1994), 
pp. 1886-1889. arXiv: hep-ph/9406319 [hep-ph] .

[150] G. R. Dvali, George Lazarides, and Q. Shafi. "Mu problem and hybrid inflation in supersymmetric SU(2)-L x SU(2)-R x U(1)-(B-L)". In: Phys. Lett. B424 (1998), pp. 259-264. arXiv: hep-ph/9710314 [hep-ph].

[151] Wilfried Buchmuller, Laura Covi, and David Delepine. "Inflation and supersymmetry breaking". In: Phys. Lett. B491 (2000), pp. 183-189. arXiv: hep-ph/0006168 [hep-ph].

[152] Laura Covi and Simone Dresti. "Time-dependent Features in the Primordial Spectrum". In: (2018). arXiv: 1803.02351 [gr-qc].

[153] Robert H. Brandenberger and Jerome Martin. "Trans-Planckian Issues for Inflationary Cosmology". In: Class. Quant. Grav. 30 (2013), p. 113001. arXiv: 1211.6753 [astro-ph.CO].

[154] Martin S. Sloth. "On the one loop corrections to inflation and the CMB anisotropies". In: Nucl. Phys. B748 (2006), pp. 149-169. arXiv: astro-ph/ 0604488 [astro-ph].

[155] Antonio Riotto and Martin S. Sloth. "On Resumming Inflationary Perturbations beyond One-loop". In: JCAP 0804 (2008), p. 030. arXiv: 0801.1845 [hep-ph].

[156] Daniel Boyanovsky. "Fermionic influence on inflationary fluctuations". In: Phys. Rev. D93.8 (2016), p. 083507. arXiv: 1602.05609 [gr-qc].

[157] Jurjen F. Koksma and Tomislav Prokopec. "Fermion Propagator in Cosmological Spaces with Constant Deceleration". In: Class. Quant. Grav. 26 (2009), p. 125003. arXiv: 0901.4674 [gr-qc].

[158] P. A. R. Ade et al. "Planck 2015 results. XVII. Constraints on primordial non-Gaussianity". In: Astron. Astrophys. 594 (2016), A17. arXiv: 1502.01592 [astro-ph.CO].

[159] C. G. Bollini and J. J. Giambiagi. "Dimensional Renormalization: The Number of Dimensions as a Regularizing Parameter". In: Nuovo Cim. B12 (1972), pp. 2026.

[160] Gerard 't Hooft and M. J. G. Veltman. "Regularization and Renormalization of Gauge Fields". In: Nucl. Phys. B44 (1972), pp. 189-213. 
It has been almost ten years since I started my academic adventure by moving from the Lago Maggiore, where I grew up, to Lake Geneva, where I was about to start studying physics. A whole new world was awaiting me, a life far from the traditions of the valleys and alpine villages I was used to.

My decision to study physics had come about a bit by chance and I took on the challenge of university studies without really thinking about what awaited me on the other side. It was not easy to start out on a new adventure in a new city, but the experience was very stimulating! Time flew by during my first years of university, positive memories of Geneva remain, a chaotic but human-scale city.

Very soon it became clear to me that I was most interested in theoretical and computational physics. During my master studies I decided to explore these fields and started to work on quantum field theory, which became my main research interest.

Towards the end of my studies, I started feeling like the time had come to leave Geneva and to embark on a new adventure! Again, I had no deep reason for choosing to continue my academic career with PhD studies in Göttingen, in Germany. It was just as luck would have it. I had been given the opportunity to continue my work on renormalization in curved space-time, which had been the subject of my master project, and that in a city known worldwide for its university research activity! I would like to thank Göttingen for the endless opportunities it has given me for my scientific and personal growth.

Now I would like to spend a few words on thanking the people who helped and supported me during the last ten years, making my journey possible. I will begin by expressing my deep and sincere gratitude to my supervisor Prof. Laura Covi who with her overwhelming passion managed to motivate me during my $\mathrm{PhD}$ studies, encouraging me to continue working on my project despite the difficulties I encountered on the way. The last phase of my doctorate was certainly the most difficult, both 
emotionally and scientifically, however, it was also the most rewarding, since I could now fully appreciate what research was all about. I would also like to express my gratitude towards Prof. Karl-Henning Rehren who allowed me to take advantage of his deep understanding of quantum field theory in many enlightening discussions. I also thank Prof. Dorothea Bahns, Chiara Entradi and Thomas-Paul Hack for their contributions to my research project and for our long and engaging discussions during the first part of my doctorate. I thank the graduate school GRK1493 and the University of Göttingen for allowing me to undertake this scientific journey and for providing the best environment for my academic training. I thank all the members of my thesis committee for showing interest in my work. Special thanks I would like to address to my master thesis supervisor Prof. Antonio Walter Riotto and to the University of Geneva, who initiated me to the wonderful world of scientific research. I thank the secretaries Gabriele Schubert, Heidi Afshar and Katrin Glormann both for their work and their unlimited patience with me.

I would like to thank my colleagues Federico, Fiona, Giorgio, Riccardo and Tobias with whom I had the honour of sharing a part of my journey. I thank my office mates Abir, Florian, Marcel, Rainer and Stephan for tolerating me over all those years. I would also like to express special thanks to my family: my uncles Dino, Ermanno, Gianluigi and Miriam, my cousins Andrea, Christian, Daniel and Marc, and to my grandparents Marina, Palmira and Pierino, to whom I dedicate this thesis. Finally, I would like to thank my parents Franco and Marinella. Thank you for supporting me in all possible ways despite not knowing exactly what I was doing or what my job was all about.

This thesis is also dedicated to my closest friends, some new, others long-standing, who have supported and encouraged me during these years. The long journey of my $\mathrm{PhD}$ studies made me understand the true importance of friendship and it is especially to my friends that I would like to express my most sincere thanks!

I thank my friends from the village of Gurro, in particular Dario, Omar and Daniel, for being part of my best childhood memories. I thank Alessio, Fiorenza and Stefania, who I met in Ascona and with whom I shared another part of my life. I have beautiful memories from our travels around Europe, a tradition I hope we will keep alive in the future. I express my special thanks to my former flat-mate Vittorio who, after a few months of shared living, turned out to be a faithful friend, always available and who has become one of the central figures of my life. I also thank my Vanzone-friends, in particular Clarissa and Ilaria, for sharing some of their summers with me. Thank you for having had the courage to cross national borders to visit me. I thank my friends from Geneva, in particular Elise, Titouan and Vincent 
for participating in an exploratory mission in Germany and especially Alexia for sharing the German adventure. In addition to the countless cities we visited, I will never forget when in Magdeburg, during our first months of the doctorate, an Italian waiter advised us to escape from Germany as soon as possible.

During the years in Göttingen I had the opportunity to see the birth of friendships that I hope to carry into the future. I would like to thank Matteo and Piero for sharing this adventure with me and for bringing some color into the days in Göttingen. In particular, I thank Matteo for introducing me to the Friûl, and for the deep philosophical and political discussions. I thank Luca for being a traveling companion with the natural gift of always seeing the bright side. Thank you for your realism and for having shared important moments of your life with me. I thank Daniela for her temperament. Surely we will be remembered in Göttingen for the countless times we were asked to calm down and make less noise. I thank Lorenzo, with whom I shared the last part of my doctorate, for his pragmatism. Thank you for having had the patience to listen when I needed it. Special thanks also to Chiara and Michael, two colleagues but, above all, two friends with whom I shared the joys and sorrows of this journey. With pleasure I recall the long walks and the even longer discussions about every aspect of life. With joy I will remember your ability to entertain, simply with your way of doing things. I wish you a happy future!

A great thank you also to the friends I met during the journeys around Europe, in particular in Corfu and Rome. I thank Robin for his friendship and for tolerating my humour, and Roman for being intrinsically fun. I would like to express my gratitude to a special friend, Alessandro, who, despite the distance, always managed to put me in a good mood and stayed by my side when I needed it. It was a pleasure to share this journey with you, thank you for the beautiful memories you left me.

The time has come to close the circle and to start planning my future, instead of letting chance decide, but with the awareness of having been on a wonderful journey that allowed me to grow and mature, not only from the scientific point of view, but also as a person.

I leave Göttingen, die Stadt, die Wissen schafft, with the confidence that you have given me the strength I need to face the future.

Many thanks to you all.

Gurro, 1st April 2018

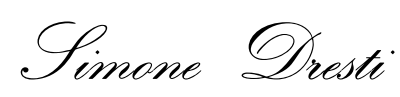


Sono passati quasi 10 anni da quando ho iniziato la mia avventura accademica passando dalle sponde del Lago Maggiore, dove sono cresciuto, a quelle del Lago Lemàno dove ho iniziato a studiare fisica. Un mondo tutto da scoprire, ben lontano dalle tradizioni delle vallate e dei borghi alpini a cui ero abituato.

Ho scelto di studiare fisica un po' per caso, un po' per fortuna. Mi sono lanciato in questa sfida senza avere troppe aspettative di quello che sarebbe successo nella mia vita. Iniziare una nuova avventura in una nuova città non è semplice, ma gli stimoli non mancano di certo! I primi anni di università sono volati. Ho solo dei ricordi positivi di Ginevra: una città caotica a misura d'uomo.

È stato chiaro fin da subito che la direzione intrapresa sarebbe stata fisica teorica e computazionale. Ho dedicato i miei due anni di magistrale cercando di approfondire questi temi e ho iniziato ad addentrarmi nella teoria quantistica dei campi, settore della fisica che oggi è diventato il tema cardine della mia ricerca.

Era giunto il momento di lasciare Ginevra e di iniziare una nuova avventura! Non c'è un vero motivo per cui ho deciso di continuare la mia carriera accademica con un dottorato a Gottinga, in Germania. È successo un po' per caso e un po' per fortuna. Ho avuto la possibilità di dare continuità al lavoro che avevo iniziato con la tesi magistrale sulla rinormalizzazione sugli spazi curvi in una città che è conosciuta in tutto il mondo per la sua attività di ricerca universitaria! Devo ringraziare Gottinga per le infinite opportunità di formazione scientifica e personale che mi ha dato.

Voglio spendere un po' di parole per ringraziare tutte le persone che mi hanno aiutato e mi sono state vicine in questi 10 anni e che hanno reso tutto questo possibile. Inizio con l'esprimere la mia profonda e sincera gratitudine alla mia relatrice Prof. Laura Covi che con la sua travolgente passione è riuscita a motivarmi in questi anni spronandomi a portare avanti il progetto senza stravolgerlo dopo le prime difficoltà. L'ultimo periodo è stato sicuramente quello emotivamente e scientificamente più difficile ma 
anche quello più gratificante dove sono riuscito ad apprezzare fino in fondo la ricerca. Vorrei esprimere la mia gratitudine anche a Prof. Karl-Henning Rehren che mi ha permesso di approfittare della sua profonda comprensione della teoria quantistica dei campi con illuminanti discussioni. Ringrazio altresì Prof. Dorothea Bahns, Chiara Entradi e Thomas-Paul Hack per aver contribuito al mio progetto di ricerca con lunghe e coinvolgenti discussioni durante la prima parte del dottorato. Ringrazio la scuola di specializzazione GRK1493 e l'Università di Gottinga per avermi permesso di intraprendere questo percorso di ricerca fornendomi tutti gli strumenti necessari per la mia formazione scientifica. Un ringraziamento speciale va al mio relatore di tesi magistrale Prof. Antonio Walter Riotto e all'Università di Ginevra per aver reso possibile l'inizio di questo lungo viaggio nel mondo della ricerca scientifica. Ringrazio tutti i membri del mio comitato di tesi per aver mostrato interesse nel mio lavoro. Ringrazio inoltre le segretarie Gabriele Schubert, Heidi Afshar e Katrin Glormann per il loro lavoro e l'indefinibile pazienza dimostrata nei miei confronti.

Un particolare grazie va ai miei colleghi Federico, Fiona, Giorgio, Riccardo e Tobias con cui ho avuto l'onore di poter condividere parte del mio cammino. Ringrazio anche i miei colleghi d'ufficio Abir, Florian, Marcel, Rainer e Stephan per avermi sopportato in questi lunghi anni. Un ringraziamento speciale va alla mia famiglia: i miei zii Dino, Ermanno, Gianluigi e Miriam, i miei cugini Andrea, Christian, Daniel e Marc, i miei nonni a cui dedico questa tesi Marina, Palmira e Pierino e infine i miei genitori Franco e Marinella. Grazie per avermi sostenuto in tutti i modi possibili senza mai avere ben in chiaro cosa stessi facendo e in cosa consistesse il mio lavoro.

Questa tesi la dedico anche agli amici più stretti, nuovi e di vecchia data che mi hanno sostenuto e incoraggiato in questi anni. Questo lungo percorso mi ha fatto capire la loro vera importanza. Un grazie sincero va soprattutto a loro!

Ringrazio gli amici del paese di Gurro, in particolare Dario, Omar e Daniel per far parte dei ricordi più belli che ho della mia infanzia. Ringrazio anche Alessio, Fiorenza e Stefania che ho conosciuto ad Ascona con cui ho condiviso parte della mia vita. Ho dei bellissimi ricordi dei diversi viaggi che abbiamo fatto in giro per l'Europa, tradizione che spero riusciremo a mantener viva in futuro. Un ringraziamento speciale va a Vittorio che dopo aver condiviso l'appartamento con me per pochi mesi ho scoperto essere un amico fedele sempre disponibile e di cui oggi non potrei fare a meno. Ringrazio anche gli amici di Vanzone, in particolare Clarissa e Ilaria, per aver condiviso con me parte delle loro estati. Grazie per aver avuto il coraggio di varcare i confini nazionali per venirmi a trovare. Ringrazio gli amici di Ginevra, in particolare Elise, Titouan e Vincent per aver partecipato alla missione esplorativa della Germania e soprattutto Alexia per aver condiviso quest'avventura in Germania. 
Oltre alle innumerevoli città che abbiamo visitato non potrò mai dimenticare quando a Magdeburgo, durante i nostri primi mesi di dottorato, un ristoratore italiano ci ha consigliato di scappare dalla Germania il prima possibile.

Durante questi anni a Gottinga ho avuto la possibilità di veder nascere delle amicizie che spero di riuscire a portare avanti anche in futuro. Ringrazio Matteo e Piero per aver condiviso con me questa avventura e aver dato un po' di colore alle giornate di Gottinga durante il mio tempo libero. In particolare, grazie Matteo per avermi fatto conoscere il Friûl e per esserti avventurato con me in profonde discussioni filosofiche e politiche. Ringrazio Luca per essere stato un compagno di viaggio con il dono naturale di riuscire a vedere il lato positivo in tutte le cose. Grazie per il tuo realismo e per aver voluto condividere con me dei momenti importanti della tua vita. Ringrazio Daniela per il suo temperamento. Sicuramente verremo ricordati a Gottinga per le innumerevoli volte che ci hanno chiesto di calmarci e di fare meno rumore. Ringrazio particolarmente Lorenzo con cui ho condiviso l'ultima parte del mio dottorato per il suo pragmatismo. Grazie per aver avuto la pazienza di ascoltarmi quando ne ho avuto bisogno. Un ringraziamento speciale va a Chiara e Michael, due colleghi ma soprattutto due amici con cui ho condiviso le gioie e i dolori di questo percorso. Ricordo con piacere le lunghe camminate e le lunghissime discussioni su ogni aspetto della vita. Mi ricorderò sempre della vostra capacità di intrattenere le persone con il vostro modo di essere. Vi auguro un futuro felice!

Un grazie speciale va anche agli amici che ho conosciuto in giro per l'Europa durante questo percorso, in particolare a Corfù e a Roma. Ringrazio Robin per la sua amicizia e per aver sopportato il mio umorismo e Roman per il suo essere intrinsecamente divertente. Un sincero grazie va ad un amico speciale, Alessandro, che nonostante la distanza è riuscito a regalarmi sempre il buon umore ed ha saputo starmi vicino quando ne ho avuto bisogno. È stato un piacere coinvolgerti in questo percorso, grazie per i bellissimi ricordi che mi hai lasciato.

Adesso è giunto il momento di chiudere il cerchio e iniziare a pianificare il mio futuro, non più per caso ma con la consapevolezza di aver fatto un bellissimo percorso che mi ha permesso di crescere e maturare non solo dal punto di vista scientifico ma anche come persona.

Lascio Gottinga, die Stadt, die Wissen schafft, con la forza di poter contare su di voi.

Grazie di cuore a tutti.

Gurro, il 1 aprile 2018

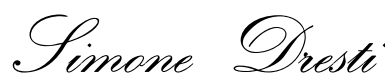


PERSONAL INFORMATION

\section{Simone Dresti}

- Philipp-Reis-Straße 9, 37075 Göttingen (Germany)

\simone.dresti@theorie.physik.uni-goettingen.de

Sex Male | Date of birth 09/06/1989 | Nationality Italian

EDUCATION AND TRAINING

02/09/2013-06/07/2018

19/09/2011-15/05/2013

15/09/2008-10/09/2011

PERSONAL SKILLS

Mother tongue(s)

Foreign language(s)

French

English

German

(a)

Digital skills 
Others:

- Markup Languages: HTML4, LaTeX(document classes: report, article, beamer)

- Office suite: LibreOffice, Microsoft Office

Driving licence

B

ADDITIONAL INFORMATION

Publications

Prizes/Scholarships

Workshop Presentations

Given Seminars

Tutorials

Visits
Laura Covi and Simone Dresti. «Time-dependent Features in the Primordial Spectrum». arXiv: 1803.02351 [gr-qc].

Simone Dresti and Antonio Riotto. «Renormalization of composite operators in time-dependent backgrounds». In: Nuclear Physics B 874.3 (2013), pp. 792-807. doi:

10.1016/j.nuclphysb.2013.06.019. arXiv: 1305.6401 [hep-ph].

2013 - RTG 1493 Scholarship, 09/2013-08/2016

2011 - Excellence Master Fellowships in Physics, 09/2011-02/2013

Workshop - 20/09/17 - Time-dependent Features in the Primordial Spectrum - Bologna Workshop Bologna (Italy)

Workshop - 03/04/17 - Time-dependent Features in the Primordial Spectrum - Göttingen Workshop Göttingen (Germany)

Workshop - 01/10/15 - Renormalization freedom in de Sitter spacetime - DESY Theory Workshop Hamburg (Germany)

Workshop - 09/05/15 - Renormalization freedom in curved spacetime - Kosmologietag - Bielefeld (Germany)

Workshop - 29/08/17 - Time-dependent Features in the Primordial Spectrum - COSMO '17 - Paris (France)

16/01/2017 - Quantum Field Theory in Curved Spacetime and Cosmological Applications Quantenfeldtheorie Forschungsseminar - University of Göttingen - Göttingen (Germany)

13/06/2016 - Renormalization in curved spacetime and Cosmological Applications Quantenfeldtheorie Forschungsseminar - University of Göttingen - Göttingen (Germany)

19/10/2015 - An overview of Renormalization in Quantum Field Theory - GRK1493 Retreat 2015 Bad Pyrmont (Germany)

05/05/2015 - Renormalization as an extension of distributions - Oberseminar on Mathematical Physics - University of Göttingen - Göttingen (Germany)

24/11/2014 - An overview of Renormalization in Curved Space-Time - Quantenfeldtheorie Forschungsseminar - University of Göttingen - Göttingen (Germany)

25/11/2013 - Composite operators in time-dependent backgrounds - Quantenfeldtheorie Forschungsseminar - University of Göttingen - Göttingen (Germany)

29/05/2013 - Renormalization of Composite Operators in time-dependent Backgrounds - University of Heidelberg - Heidelberg (Germany)

Tutor - Exercise lecturer for the course of Quantenmechanik II, 11/2017-12/2017, WiSe17/18

Tutor - Exercise lecturer for the course Mathematische Methoden der Physik II, 10/2014-03/2015, WiSe14/15

Tutor - Exercise lecturer for the course of Quantenmechanik I, 04/2014-10/2014, SoSe14

Visitor at Unige - 07/06/16-08/06/16, Geneva (Switzerland)

Short-term visitor at CERN, 09/05/16-10/06/16, Geneva (Switzerland) 
Conferences/Workshops Attended

Lectures Attended

Seminars Attended
Workshop - 05/02/18-06/02/18 - Job Hunting and Application Skills - Göttingen (Germany)

Workshop - 18/09/17-21/09/17 - Post-inflationary String Cosmology - Bologna (Italy)

Workshop - 28/08/17-01/09/17 - 21st annual International Conference on Particle Physics and Cosmology - Paris (France)

Workshop - 03/04/17-05/04/17 - Open Questions in Particle Physics and Cosmology - Göttingen (Germany)

Workshop - 15/01/16-16/01/16 - Fundation and Constructive Aspects of QFT - Göttingen (Germany) Retreat - 19/10/15-20/10/15 - GRK1493 Retreat 2015 - Bad Pyrmont (Germany)

Workshop - 29/09/15-02/10/15 - Physics at the LHC and beyond - DESY-Hamburg (Germany)

School - 16/05/15-29/05/15 - First ICTP Advanced School on Cosmology - Trieste (Italy)

Workshop - 07/05/15-08/05/15 - 10. Kosmologietag - Bielefeld (Germany)

School - 01/12/14-04/12/14 - Winter School on Theoretical Physics - Odense (Danemark)

Workshop - 14/11/14-15/11/14 - Foundations and Constructive Aspects of QFT - Goslar (Germany)

Workshop - 23/09/14-26/09/14 - Particle Cosmology after Planck - DESY-Hamburg (Germany)

School/Workshop - 03/09/14-14/09/14 - The Standard Model and Beyond - Corfù (Greece)

Workshop - 27/01/14-29/01/14 - Inflation after Planck - DESY-Hamburg (Germany)

Workshop - 14/11/13-16/11/13 - Fundation and Constructive Aspects of QFT - Göttingen (Germany)

School - 07/10/13-11/10/13 - Autumn School on Particle Physics and Cosmology - Göttingen (Germany)

Retreat - 04/10/13-06/10/13 - GRK1493 Retreat 2013 - Goslar (Germany)

Information and Physics given by Stefan Kehrein, WiSe15/16

Liquid State Physics given by Oliver Bäumchen, WiSe14/15

Good Scientific Practice given by Karl-Henning Rehren, WiSe14/15

Introduction to Supersymmetry given by Riccardo Catena, WiSe13/14

Symmetries in Modern Physics given by Müller-Hoissen, WiSe13/14

Quantenfeldtheorie Forschungsseminar - University of Göttingen - WiSe2013/14-WiSe17/18

Invisibles/Elusives - European ITN project - WiSe2013/14-WiSe17/18

Born-Hilbert Seminar - University of Göttingen - WiSe2013/14-WiSe17/18

Cosmology Seminar - University of Göttingen - WiSe2013/14-WiSe16/17

GRK1493 Seminar - University of Göttingen - WiSe2013/14-SoSe16 